Xu, Zhang, Ingoglia, Allais, Dechert-Schmitt, Singer and Morken Supporting Information

\title{
Construction of Azacycles by Intramolecular Amination of Organoboronates and Organobis(boronates)
}

\author{
Peilin Xu, Mingkai Zhang, Bryan Ingoglia, Christophe Allais, Anne-Marie R. Dechert-Schmitt, \\ Robert A. Singer, and James P. Morken* \\ Department of Chemistry, Merkert Chemistry Center, Boston College, Chestnut Hill, \\ Massachusetts 02467, United States \\ Pfizer Worldwide Research and Development, Eastern Point Road, Groton, Connecticut 06340, \\ United States
}

\section{Supporting Information}

Table of Contents

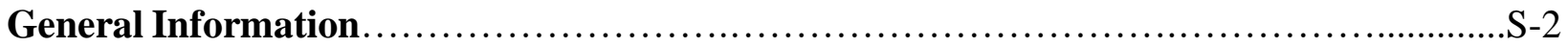

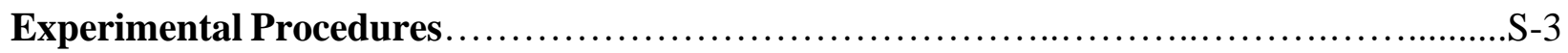

I. Representative procedures for preparation of starting materials .....................S-3

II. Representative Procedure for Intramolecular Amination ......................................S-6

III. Procedures for Preparation of substrates ................................ 16

IV. Procedures and Characterization for Intramolecular Amination Product ..........S-17

V. Synthesis of Enantiomerically Enriched Compounds and Testing of Stereospecificity

References.

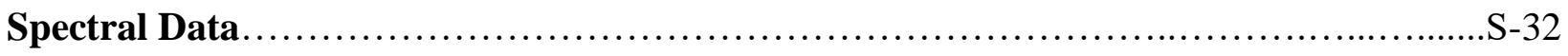




\section{General Information}

${ }^{1} \mathrm{H}$ NMR spectra were recorded on either a Varian Gemini-600 (600 MHz), Inova-400 (400 $\mathrm{MHz})$ or Varian Inova-500 (500 MHz) spectrometer. Chemical shifts are reported in ppm with the solvent resonance as the internal standard $\left(\mathrm{CDCl}_{3}: 7.26 \mathrm{ppm}\right)$. Data are reported as follows: chemical shift, integration, multiplicity $(s=$ singlet, $d=$ doublet, $\mathrm{t}=$ triplet, $\mathrm{q}=$ quartet, $\mathrm{p}=$ pentet, $\mathrm{m}=$ multiplet), and coupling constants $(\mathrm{Hz}) \cdot{ }^{13} \mathrm{C}$ NMR spectra were recorded on either a Varian Gemini-600 (150 MHz) or a Varian Inova-500 (125 MHz) spectrometer with complete proton decoupling. Chemical shifts are reported in ppm with the solvent resonance as the internal standard $\left(\mathrm{CDCl}_{3}\right.$ : $\left.77.16 \mathrm{ppm}\right) .{ }^{11} \mathrm{~B} \mathrm{NMR}$ spectra were recorded on a Varian Gemini-500 (128 MHz) spectrometer. Infrared (IR) spectra were recorded on a Bruker alpha-P Spectrometer. Frequencies are reported in wavenumbers $\left(\mathrm{cm}^{-1}\right)$ as follows: strong (s), broad (br), medium (m), and weak (w). Optical rotations were measured on a Rudolph Analytical Research Autopol IV Polarimeter. Highresolution mass spectrometry (DART+) was performed at the Mass Spectrometry Facility, Boston College, Chestnut Hill, MA. Purification was performed using forced flow (flash chromatography) on silica gel $\left(\mathrm{SiO}_{2}, 230\right.$ x $\left.450 \mathrm{Mesh}\right)$ purchased from Silicycle. Thin layer chromatography (TLC) was performed on $25 \mu \mathrm{m}$ silica gel aluminum backed plates from Silicycle. Visualization was performed using ultraviolet light $(254 \mathrm{~nm})$, ceric ammonium molybdate (CAM) in ethanol, basic potassium permanganate or ninhydrin and acetic acid in ethanol.

Analytical chiral supercritical fluid chromatography (SFC) was performed on a TharSFC Method Station II equipped with Waters 2998 Photodiode Array Detector with isopropanol as the modifier. HPLC measurements were carried out on a Shimadzu HPLC system.

All reactions were conducted in oven- or flame-dried glassware under an inert atmosphere of nitrogen or argon unless otherwise specified. Heated reactions are conducted in a mineral oil bath on a hot plate equipped with a temperature probe. Tetrahydrofuran (THF), diethyl ether $\left(\mathrm{Et}_{2} \mathrm{O}\right)$, dichloromethane $(\mathrm{DCM})$ and toluene were purified using Pure Solv MD-4 solvent purification system, from Innovative Technology, Inc., by passing the solvent through two activated alumina columns after purging with nitrogen. Potassium tert-butoxide was purchased from Oakwood Chemicals. Tris(dibenzylidineacetone)platinum(0) $\left(\mathrm{Pt}(\mathrm{dba})_{3}\right)$ was synthesized following literature methods. ${ }^{1}$ (3aS,8aS)-(+)-4,4,8,8-tetrakis(3,5-diethylphenyl)tetrahydro-2,2dimethyl-6-phenyl-1,3-dioxolo[4,5-e]dioxaphosphepine $((S, S)$-3,5-diethylphenylTADDOLPPh) was synthesized from D-(-)-tartaric acid (obtained from Sigma Aldrich) following literature methods. ${ }^{2}$ All other reagents were purchased from Sigma Aldrich, Alfa Aesar, Oakwood Chemicals, Combi-Blocks, or Acros Organics and used without further purification. 


\section{Experimental Procedures}<smiles>CONC(=O)OC(C)(C)C</smiles>

tert-butyl methoxycarbamate (S-1) The title compound was prepared according to the procedure reported in the patent literature. ${ }^{3}$ All spectral data was in accordance with previously published results.<smiles>C=CC(CO)c1ccccc1</smiles>

2-phenylbut-3-en-1-ol (S-2) The title compound was prepared according to the procedure reported in the literature. ${ }^{4}$ All spectral data was in accordance with previously published results.<smiles>C=CCC(CO)c1ccccc1</smiles>

2-phenylpent-4-en-1-ol (S-3) The title compound was prepared according to the procedure reported in the literature. ${ }^{5}$ All spectral data was in accordance with previously published results.

\section{Representative procedures for preparation of starting materials}

\section{General procedure A: $S_{N} 2$ substitution with tert-butyl methoxycarbamate}

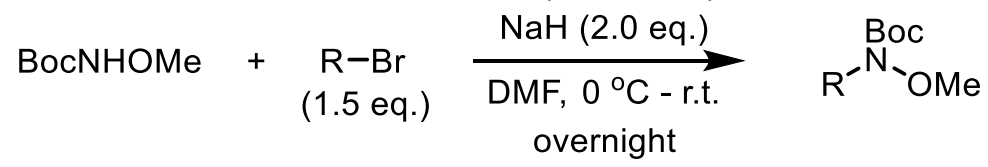

In a round bottom flask, tert-butyl methoxycarbamate (S-1) $(20 \mathrm{mmol}, 1.0$ equiv.) was added dropwise carefully to a suspension of sodium hydride (40 mmol, 2.0 equiv.) in DMF (50 mL) at 0 ${ }^{\circ} \mathrm{C}$. After addition was complete, the reaction was allowed to warm up to room temperature and stirred for 1 hour. The reaction was cooled down to $0{ }^{\circ} \mathrm{C}$, and the corresponding alkyl bromide (30 mmol, 1.5 equiv.) was added to the reaction mixture, which was then warmed to room temperature and allowed to stir overnight. The reaction was quenched carefully with saturated ammonium chloride solution $(20 \mathrm{~mL})$, diluted with diethyl ether $(100 \mathrm{~mL})$, and transferred to a separatory funnel. The organic phase was washed with DI water $(4 \times 10 \mathrm{~mL})$ and then with brine $(10 \mathrm{~mL})$. The organic layer was dried over anhydrous sodium sulfate and concentrated under reduced pressure. The pure products were isolated with $\mathrm{SiO}_{2}$ chromatography. 
General procedure B: Mitsunobu reaction with tert-butyl methoxycarbamate

$$
\begin{aligned}
& \text { DBAD (1.5 eq.) }
\end{aligned}
$$

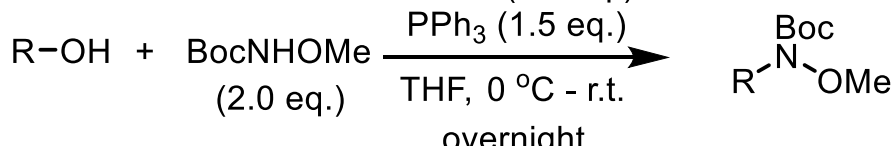

This procedure is adapted from literature. ${ }^{6}$ In a round bottom flask, the corresponding alcohol (10 mmol, 1.0 equiv.), triphenyl phosphine (15 mmol, 1.5 equiv.) and tert-butyl methoxycarbamate (S-1) (20 mmol, 2.0 equiv) were dissolved in THF $(20 \mathrm{~mL})$. The reaction mixture was cooled to 0 ${ }^{\circ} \mathrm{C}$, and di-tert-butyl azodicarboxylate $(15 \mathrm{mmol}, 1.5$ equiv.) in THF (15 mL) was added to the reaction mixture, which was then warmed to room temperature and stirred overnight. At this time, the reaction mixture was opened to the air and the solvent was removed under reduced pressure. The pure products were isolated with $\mathrm{SiO}_{2}$ chromatography.

General procedure C: Diboration of alkenes

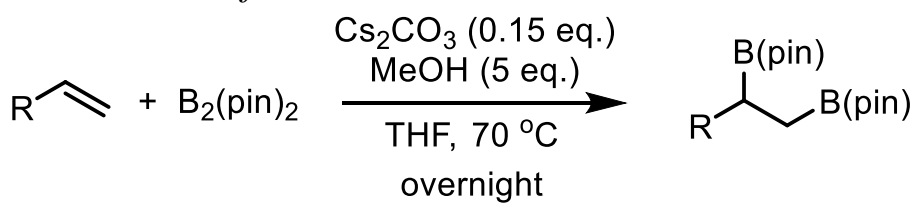

This procedure is adapted from literature. ${ }^{7}$ A round bottom flask was charged with cesium carbonate (1.5 mmol, 0.15 equiv.) and bis(pinacolato)diboron (11 mmol, 1.1 equiv.). THF (30 mL) and methanol (50 mmol, 5.0 equiv.) was added, followed by the corresponding alkene (10 mmol, 1.0 equiv.). The reaction was heated at $70{ }^{\circ} \mathrm{C}$ overnight. At this time, the reaction mixture was opened to the air and the solvent was removed under reduced pressure. The pure products were isolated with $\mathrm{SiO}_{2}$ chromatography.

General procedure D: Hydroboration of alkenes.

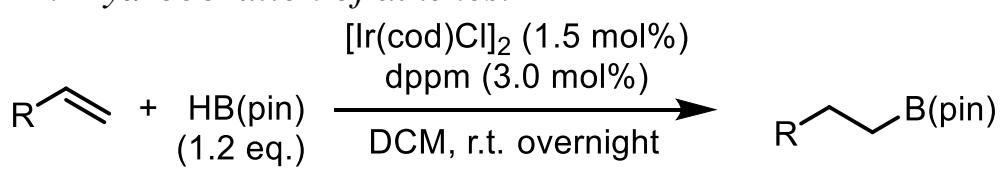

This procedure is adapted from literature. ${ }^{8}$ An oven-dried vial was loaded with $[\operatorname{Ir}(\operatorname{cod}) \mathrm{Cl}]_{2}(0.015$ mmol, $1.5 \mathrm{~mol} \%$ ) and bis(diphenylphosphino)methane (0.03 mmol, $3 \mathrm{~mol} \%)$. Anhydrous DCM (4 $\mathrm{mL}$ ) was added, followed by the corresponding alkene (1.0 mmol, 1.0 equiv.) and pinacol borane (1.2 mmol, 1.2 equiv.). The mixture was stirred at room temperature overnight, then quenched with water $(2 \mathrm{~mL})$, extracted with diethyl ether $(3 \times 10 \mathrm{~mL})$ and dried over anhydrous sodium sulfate. After removal of solvent under reduced pressure, the pure products were isolated with $\mathrm{SiO}_{2}$ chromatography.

General procedure E: Suzuki-Miyaura cross-coupling of 1,2-bisboronates

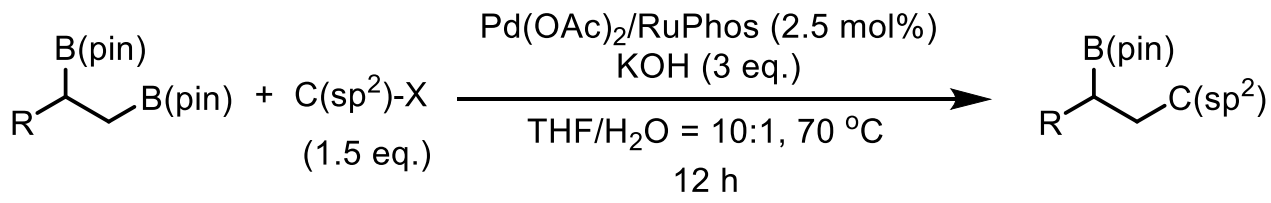

This procedure is adapted from literature. ${ }^{9}$ In the glovebox, an oven dried vial was charged with the corresponding 1,2-bisboronate (1.0 mmol, 1.0 equiv.), solid potassium hydroxide ( 3 mmol, 3 
equiv.), $\mathrm{Pd}(\mathrm{OAc})_{2} / \mathrm{RuPhos}(0.025 \mathrm{mmol}, 2.5 \mathrm{~mol} \%)$ as a $1: 1$ solution in THF $(1 \mathrm{~mL}), \mathrm{THF}(9 \mathrm{~mL})$ and electrophile (1.5 mmol, 1.5 equiv.). The vial was sealed, removed from the glovebox, and $\mathrm{H}_{2} \mathrm{O}$ (sparged with $\mathrm{N}_{2}$ for $30 \mathrm{~min}, 1.0 \mathrm{~mL}$ ) was added through the teflon septum cap. The vial was heated to $70^{\circ} \mathrm{C}$ and stirred for $12 \mathrm{~h}$. At this time, the vial was cooled down to room temperature and opened to air, and the reaction mixture was diluted with ethyl acetate and the aqueous and organic layers were separated. The aqueous layer was extracted with ethyl acetate $(3 \times 10 \mathrm{~mL})$ and the combined organics were dried over anhydrous sodium sulfate. After removal of solvent under reduced pressure, the pure products were isolated with $\mathrm{SiO}_{2}$ chromatography. 


\section{Procedures for Preparation of substrates}<smiles>C=CCCN(OC)C(=O)OCc1ccccc1</smiles>
according to General procedure $A$ with 4-bromobut-1-ene (3.05 mL, $30 \mathrm{mmol}, 1.5$ equiv.), tertbutyl methoxycarbamate (S-1) (2.94 g, 20 mmol, 1.0 equiv.), sodium hydride (1.07 g, 90\% purity, $40 \mathrm{mmol}, 2$ equiv.) in DMF (50 mL). The compound was purified with $\mathrm{SiO}_{2}$ chromatography $(10 \%$ ethyl acetate in hexane, stain in $\left.\mathrm{KMnO}_{4}\right)$ as a colorless oil $(3.41 \mathrm{~g}, 85 \%)$.

${ }^{1} \mathbf{H}$ NMR $\left(600 \mathrm{MHz}, \mathrm{CDCl}_{3}\right) \delta 5.82-5.73(\mathrm{~m}, 1 \mathrm{H}), 5.09(\mathrm{dd}, J=17.1,1.7 \mathrm{~Hz}, 1 \mathrm{H}), 5.03(\mathrm{dd}, J=$ 10.3, 1.7 Hz, 1H), 3.66 (s, 3H), $3.51-3.46(\mathrm{~m}, 2 \mathrm{H}), 2.35$ (q, J = 7.1, 7.0, 7.0 Hz, 2H), 1.47 (s, 9H). ${ }^{13} \mathrm{C} \mathrm{NMR}\left(151 \mathrm{MHz}, \mathrm{CDCl}_{3}\right) \delta 156.4,135.4,116.8,81.3,62.3,48.7,31.6,28.4$. IR (neat) $v_{\max } 2977(\mathrm{w}), 2934(\mathrm{w}), 1727(\mathrm{~m}), 1700$ (s), $1642(\mathrm{w}), 1467(\mathrm{w}), 1436(\mathrm{w}), 1366$ (s), $1320(\mathrm{w})$, $1287(\mathrm{w}), 1252(\mathrm{~m}), 1220(\mathrm{w}), 1147$ (s), $1074(\mathrm{~m}), 1034(\mathrm{w}), 993(\mathrm{~m}), 914(\mathrm{~m}), 858(\mathrm{w}), 837(\mathrm{w})$, $766(\mathrm{~m}), 630(\mathrm{w}), 564(\mathrm{w}) \mathrm{cm}^{-1}$. HRMS (DART+) for $\mathrm{C}_{10} \mathrm{H}_{20} \mathrm{NO}_{3}[\mathrm{M}+\mathrm{H}]^{+}$: Calc'd: 202.1438, found: 202.1440 .<smiles>C=CCCCN(C)C(=O)OCc1ccccc1</smiles>

tert-butyl methoxy(pent-4-en-1-yl)carbamate (25) The reaction was performed according to General procedure A with 5-bromopent-1-ene (3.55 mL, $30 \mathrm{mmol}, 1.5$ equiv.), tert-butyl methoxycarbamate (S-1) (2.94 g, 20 mmol, 1.0 equiv.), sodium hydride (1.07 g, 90\% purity, 40 mmol, 2 equiv.) in DMF (50 mL). The compound was purified with $\mathrm{SiO}_{2}$ chromatography (10\% ethyl acetate in hexane, stain in $\left.\mathrm{KMnO}_{4}\right)$ as a colorless oil $(3.15 \mathrm{~g}, 73 \%)$.

${ }^{1} \mathbf{H}$ NMR $\left(600 \mathrm{MHz}, \mathrm{CDCl}_{3}\right) \delta 5.82-5.72(\mathrm{~m}, 1 \mathrm{H}), 4.99(\mathrm{dq}, J=17.2,1.7,1.7,1.7 \mathrm{~Hz}, 1 \mathrm{H}), 4.97$ $-4.91(\mathrm{~m}, 1 \mathrm{H}), 3.63(\mathrm{~s}, 3 \mathrm{H}), 3.42-3.36(\mathrm{~m}, 2 \mathrm{H}), 2.09-2.00(\mathrm{~m}, 2 \mathrm{H}), 1.70-1.64(\mathrm{~m}, 2 \mathrm{H}), 1.46$ $(\mathrm{s}, 9 \mathrm{H}) .{ }^{13} \mathbf{C} \mathbf{N M R}\left(151 \mathrm{MHz}, \mathrm{CDCl}_{3}\right) \delta 156.3,137.9,115.0,81.1,62.2,48.5,30.9,28.4,26.3$. IR (neat) $v_{\max } 2977(\mathrm{w}), 2934(\mathrm{w}), 1725(\mathrm{~m}), 1701(\mathrm{~s}), 1641(\mathrm{w}), 1456(\mathrm{w}), 1438(\mathrm{w}), 1391(\mathrm{~m}), 1366$ (s), $1282(\mathrm{~m}), 1253(\mathrm{~m}), 1158$ (s), $1080(\mathrm{~m}), 992(\mathrm{w}), 911(\mathrm{w}), 852(\mathrm{w}), 825(\mathrm{w}), 765(\mathrm{w}), 627(\mathrm{w})$ $\mathrm{cm}^{-1}$. HRMS (DART+) for $\mathrm{C}_{11} \mathrm{H}_{22} \mathrm{NO}_{3}[\mathrm{M}+\mathrm{H}]^{+}$: Calc'd: 216.1594, found: 216.1604.<smiles>C=CCCCCN(OC)C(=O)OCc1ccccc1</smiles>

tert-butyl hex-5-en-1-yl(methoxy)carbamate $(\mathbf{S}-4)$ The reaction was performed according to General procedure A with 6-bromohex-1-ene (4.02 mL, $30 \mathrm{mmol}, 1.5$ equiv.), tert-butyl methoxycarbamate (S-1) (2.94 g, $20 \mathrm{mmol}, 1.0$ equiv.), sodium hydride (1.07 g, $90 \%$ purity, 40 mmol, 2 equiv.) in DMF (50 mL). The compound was purified with $\mathrm{SiO}_{2}$ chromatography (10\% ethyl acetate in hexane, stain in $\left.\mathrm{KMnO}_{4}\right)$ as a colorless oil $(4.44 \mathrm{~g}$, 97\%). ${ }^{1}$ H NMR $\left(600 \mathrm{MHz}, \mathrm{CDCl}_{3}\right) \delta 5.77-5.67(\mathrm{~m}, 1 \mathrm{H}), 4.94(\mathrm{~d}, J=17.2 \mathrm{~Hz}, 1 \mathrm{H}), 4.88(\mathrm{~d}, J=10.1$ $\mathrm{Hz}, 1 \mathrm{H}), 3.60$ (s, 3H), $3.36(\mathrm{t}, J=7.2 \mathrm{~Hz}, 2 \mathrm{H}), 2.01(\mathrm{q}, J=6.5 \mathrm{~Hz}, 2 \mathrm{H}), 1.59-1.52(\mathrm{~m}, 2 \mathrm{H}), 1.43$ (s, 9H), $1.38-1.30(\mathrm{~m}, 2 \mathrm{H}) .{ }^{13} \mathbf{C}$ NMR $\left(151 \mathrm{MHz}, \mathrm{CDCl}_{3}\right) \delta 156.3,138.5,114.6,81.0,62.1,48.8$, 33.4, 28.3, 26.6, 26.0. IR (neat) $v_{\max } 3076(\mathrm{w}), 2976$ (w), 2933 (w), 2861 (w), 2816 (w), 1700 (s), 
1640 (w), 1456 (w), 1437 (w), 1391 (m), 1366 (s), 1294 (w), 1253 (m), 1095 (w), 1060 (w), 992 (m), 909 (m), 859 (w), 765 (m), 632 (w) cm $\mathrm{cm}^{-1}$. HRMS (DART+) for $\mathrm{C}_{12} \mathrm{H}_{24} \mathrm{NO}_{3}[\mathrm{M}+\mathrm{H}]^{+}$: Calc'd: 230.1751, found: 230.1753 .<smiles>C=CC(CN(OC)C(=O)OCc1ccccc1)c1ccccc1</smiles>

tert-butyl methoxy(2-phenylbut-3-en-1-yl)carbamate (S-5) The reaction was performed according to General procedure B with 2-phenylbut-3-en-1-ol (S-2) (1.48 g, $10 \mathrm{mmol}$, 1.0 equiv.), tert-butyl methoxycarbamate (S-1) (2.95 g, $20 \mathrm{mmol}, 2.0$ equiv), triphenyl phosphine (3.92 g, $15 \mathrm{mmol}, 1.5$ equiv.) and di-tert-butyl azodicarboxylate (3.45 g, $15 \mathrm{mmol}, 1.5 \mathrm{equiv}$.) in THF (35 mL). The compound was purified with $\mathrm{SiO}_{2}$ chromatography (10\% ethyl acetate in hexane, stain in $\left.\mathrm{KMnO}_{4}\right)$ as a colorless oil (1.10 g, 40\%).

${ }^{1} \mathbf{H}$ NMR $\left(600 \mathrm{MHz}, \mathrm{CDCl}_{3}\right) \delta 7.33-7.27(\mathrm{~m}, 2 \mathrm{H}), 7.25-7.20(\mathrm{~m}, 3 \mathrm{H}), 6.07-5.98(\mathrm{~m}, 1 \mathrm{H}), 5.15$ - $5.10(\mathrm{~m}, 2 \mathrm{H}), 3.84-3.75(\mathrm{~m}, 2 \mathrm{H}), 3.74-3.67(\mathrm{~m}, 1 \mathrm{H}), 3.61(\mathrm{~s}, 3 \mathrm{H}), 1.41(\mathrm{~s}, 9 \mathrm{H}) .{ }^{13} \mathbf{C}$ NMR $\left(126 \mathrm{MHz}, \mathrm{CDCl}_{3}\right) \delta 155.9,141.6,139.2,128.7,128.2,126.8,116.5,81.3,62.1,53.8,47.8,28.4$. IR (neat) $v_{\max } 2977$ (w), 2932 (w), 1722 (s), 1701 (s), 1638 (w), 1601 (w), 1493 (w), 1476 (w), 1454 (w), 1391 (w), 1366 (s), 1304 (w), 1228 (w), 1165 (s), 1140 (w), 1087 (w), 1075 (w), 1032 (w), 997 (w), 917 (w), 860 (w), 754 (w), 700 (s), 542 (w) cm ${ }^{-1}$. HRMS (DART+) for $\mathrm{C}_{16} \mathrm{H}_{24} \mathrm{NO}_{3}$ $[\mathrm{M}+\mathrm{H}]^{+}:$Calc'd:278.1751, found: 278.1755 .<smiles>C=CCC(CN(OC)C(=O)OCc1ccccc1)c1ccccc1</smiles>

tert-butyl methoxy(2-phenylpent-4-en-1-yl)carbamate (S-6) The reaction was performed according to General procedure B with 2-phenylpent-4-en-1-ol (S-3) (1.62 g, $10 \mathrm{mmol}$, 1.0 equiv.), tert-butyl methoxycarbamate (S-1) (2.95 g, $20 \mathrm{mmol}, 2.0$ equiv), triphenyl phosphine (3.92 g, 15 mmol, 1.5 equiv.) and di-tert-butyl azodicarboxylate (3.45 g, 15 mmol, 1.5 equiv.) in THF (35 mL). The compound was purified with $\mathrm{SiO}_{2}$ chromatography (10\% ethyl acetate in hexane, stain in $\left.\mathrm{KMnO}_{4}\right)$ as a colorless oil $(1.45 \mathrm{~g}, 50 \%) .{ }^{1} \mathbf{H} \mathbf{~ N M R}\left(600 \mathrm{MHz}, \mathrm{CDCl}_{3}\right) \delta 7.31-$ $7.27(\mathrm{~m}, 2 \mathrm{H}), 7.22-7.18(\mathrm{~m}, 3 \mathrm{H}), 5.69-5.61(\mathrm{~m}, 1 \mathrm{H}), 5.01-4.96(\mathrm{~m}, 1 \mathrm{H}), 4.95-4.91(\mathrm{~m}, 1 \mathrm{H})$, $3.72-3.61(\mathrm{~m}, 2 \mathrm{H}), 3.56(\mathrm{~s}, 3 \mathrm{H}), 3.14-3.09(\mathrm{~m}, 1 \mathrm{H}), 2.49-2.36(\mathrm{~m}, 2 \mathrm{H}), 1.39(\mathrm{~s}, 9 \mathrm{H}) .{ }^{13} \mathbf{C ~ N M R}$ $\left(126 \mathrm{MHz}, \mathrm{CDCl}_{3}\right) \delta 155.8,142.3,136.2,128.4,128.2,126.6,116.5,81.1,61.9,54.1,43.6,37.9$, 28.3. IR (neat) $v_{\max } 2976(\mathrm{w}), 2931$ (w), 1720 (s), 1700 (s), 1640 (w), 1603 (s), 1494 (s), 1476 (s), 1453 (m), 1438 (m), 1391 (m), 1366 (s), 1228 (m), 1162 (s), 1127 (m), 1076 (m), 1033 (w), 994 (w), 912 (m), 855 (w), 757 (m), 699 (s) cm ${ }^{-1}$. HRMS (DART+) for $\mathrm{C}_{17} \mathrm{H}_{26} \mathrm{NO}_{3}[\mathrm{M}+\mathrm{H}]^{+}$: Calc'd: 292.1907, found: 292.1911. 


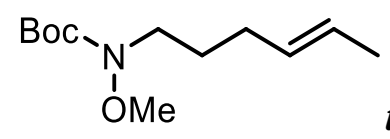

tert-butyl (E)-hex-4-en-1-yl(methoxy)carbamate (29) The reaction was performed according to General procedure B with $(E)$-hex-4-en-1-ol (1.00 g, 10 mmol, 1.0 equiv.), tert-butyl methoxycarbamate (S-1) (3.93 g, $30 \mathrm{mmol}, 3.0$ equiv), triphenyl phosphine (5.23 g, 20 mmol, 2.0 equiv.) and di-tert-butyl azodicarboxylate (4.60 g, $20 \mathrm{mmol}, 2.0$ equiv.) in THF (35 $\mathrm{mL})$. The compound was purified with $\mathrm{SiO}_{2}$ chromatography (10\% ethyl acetate in hexane, stain in $\left.\mathrm{KMnO}_{4}\right)$ as a colorless oil $(1.26 \mathrm{~g}, 54 \%) .{ }^{1} \mathbf{H} \mathbf{~ N M R}\left(500 \mathrm{MHz}, \mathrm{CDCl}_{3}\right) \delta 5.49-5.32(\mathrm{~m}, 2 \mathrm{H})$, $3.65(\mathrm{~s}, 3 \mathrm{H}), 3.44-3.35(\mathrm{~m}, 2 \mathrm{H}), 2.06-1.91(\mathrm{~m}, 2 \mathrm{H}), 1.71-1.57(\mathrm{~m}, 5 \mathrm{H}), 1.47(\mathrm{~s}, 9 \mathrm{H}) .{ }^{13} \mathbf{C ~ N M R}$ $\left(126 \mathrm{MHz}, \mathrm{CDCl}_{3}\right) \delta 156.4,130.4,125.6,81.1,62.3,48.7,29.8,28.4,27.0,18.0$. IR (neat) $v_{\max }$ 2977 (w), 2935 (w), 2162 (w), 1724 (m), 1703 (s), 1456 (w), 1438 (w), 1392 (m), 1367 (s), 1280 (w), $1256(\mathrm{w}), 1166(\mathrm{~s}), 966(\mathrm{w}) \mathrm{cm}^{-1}$. HRMS (DART+) for $\mathrm{C}_{12} \mathrm{H}_{24} \mathrm{NO}_{3}[\mathrm{M}+\mathrm{H}]^{+}$: Calc'd: 230.1751, found: 230.1761 .<smiles>CON(CC(CCBr)c1ccccc1)C(=O)OCc1ccccc1</smiles>

tert-butyl methoxy(2-phenyl-4-(4,4,5,5-tetramethyl-1,3,2dioxaborolan-2-yl)butyl)carbamate (S-7) The reaction was performed according to General procedure $D$ with tert-butyl methoxy(2-phenylbut-3-en-1-yl)carbamate (S-5) (0.28 g, $1.0 \mathrm{mmol}$, 1.0 equiv.), $[\operatorname{Ir}(\operatorname{cod}) \mathrm{Cl}]_{2}(10.1 \mathrm{mg}, 0.015 \mathrm{mmol}, 1.5 \mathrm{~mol} \%)$, bis(diphenylphosphino)methane (11.5 $\mathrm{mg}, 0.03 \mathrm{mmol}, 3 \mathrm{~mol} \%)$, pinacol borane $(0.17 \mathrm{~mL}, 1.2 \mathrm{mmol}, 1.2$ equiv.) in DCM (4 mL). The compound was purified with $\mathrm{SiO}_{2}$ chromatography (10\% ethyl acetate in hexane, stain with CAM) as a colorless oil $(0.33 \mathrm{~g}, 82 \%) .{ }^{1} \mathbf{H}$ NMR $\left(600 \mathrm{MHz}, \mathrm{CDCl}_{3}\right) \delta 7.28-7.22(\mathrm{~m}, 2 \mathrm{H}), 7.20-7.13$ $(\mathrm{m}, 3 \mathrm{H}), 3.63(\mathrm{~d}, J=7.5 \mathrm{~Hz}, 2 \mathrm{H}), 3.54(\mathrm{~s}, 3 \mathrm{H}), 2.99-2.90(\mathrm{~m}, 1 \mathrm{H}), 1.85-1.77(\mathrm{~m}, 1 \mathrm{H}), 1.69-$ $1.58(\mathrm{~m}, 1 \mathrm{H}), 1.36(\mathrm{~s}, 9 \mathrm{H}), 1.18(\mathrm{~s}, 12 \mathrm{H}), 0.67-0.56(\mathrm{~m}, 2 \mathrm{H}) .{ }^{13} \mathbf{C} \mathbf{N M R}\left(126 \mathrm{MHz}, \mathrm{CDCl}_{3}\right) \delta$ $155.8,142.6,128.3,128.3,126.4,82.9,80.9,61.8,54.6,46.0,28.2,28.1,27.8,24.9,24.8$, 9.1. IR (neat) $v_{\max } 2976(\mathrm{w}), 2931(\mathrm{w}), 1720(\mathrm{w}), 1702(\mathrm{w}), 1453(\mathrm{w}), 1366(\mathrm{~s}), 1318(\mathrm{~m}), 1236(\mathrm{w}), 1169$ (m), 1145 (s), 1093 (w), 968 (w), 859 (w), 847 (w), 757 (w), 700 (w) cm $\mathrm{cm}^{-1}$. HRMS (DART+) for $\mathrm{C}_{22} \mathrm{H}_{37} \mathrm{BNO}_{5}[\mathrm{M}+\mathrm{H}]^{+}$: Calc'd: 406.2759, found: 406.2756 .<smiles>CON(CC(CCCBr)c1ccccc1)C(=O)OC(C)(C)C</smiles>

tert-butyl methoxy(2-phenyl-5-(4,4,5,5-tetramethyl-1,3,2dioxaborolan-2-yl)pentyl)carbamate (S-8) The reaction was performed according to General procedure $D$ with tert-butyl methoxy(2-phenylpent-4-en-1-yl)carbamate (S-6) (0.29 g, $1.0 \mathrm{mmol}$, 1.0 equiv.), [ $\mathrm{Ir}(\mathrm{cod}) \mathrm{Cl}]_{2}(10.1 \mathrm{mg}, 0.015 \mathrm{mmol}, 1.5 \mathrm{~mol} \%)$, bis(diphenylphosphino)methane (11.5 $\mathrm{mg}, 0.03 \mathrm{mmol}, 3 \mathrm{~mol} \%)$, pinacol borane (0.17 mL, $1.2 \mathrm{mmol}, 1.2$ equiv.) in DCM (4 mL). The compound was purified with $\mathrm{SiO}_{2}$ chromatography (10\% ethyl acetate in hexane, stain with CAM) as a colorless oil $(0.30 \mathrm{~g}, 71 \%) .{ }^{1} \mathbf{H}$ NMR $\left(600 \mathrm{MHz}, \mathrm{CDCl}_{3}\right) \delta 7.29-7.23(\mathrm{~m}, 2 \mathrm{H}), 7.21-7.12$ $(\mathrm{m}, 3 \mathrm{H}), 3.62-3.55(\mathrm{~m}, 2 \mathrm{H}), 3.54(\mathrm{~s}, 3 \mathrm{H}), 3.03-2.93(\mathrm{~m}, 1 \mathrm{H}), 1.71-1.55(\mathrm{~m}, 2 \mathrm{H}), 1.36(\mathrm{~s}, 9 \mathrm{H})$, 
$1.31-1.24(\mathrm{~m}, 2 \mathrm{H}), 1.19(\mathrm{~s}, 12 \mathrm{H}), 0.77-0.66(\mathrm{~m}, 2 \mathrm{H}) .{ }^{13} \mathrm{C}$ NMR $\left(151 \mathrm{MHz}, \mathrm{CDCl}_{3}\right) \delta 155.9$, 143.0, 128.4, 128.3, 126.4, 83.0, 81.0, 61.9, 55.1, 43.9, 36.1, 28.3, 24.9, 21.9, 11.3. IR (neat) $v_{\max }$ 2976 (w), 2931 (w), 1721 (m), 1701 (m), 1453 (w), 1366 (s), 1320 (m), 1227 (m), 1161 (m), 1143 (s), 1093 (m), 1074 (m), 1030 (w), 992 (w), 968 (m), 846 (m), 756 (m), 700 (s), 543 (w) cm ${ }^{-1}$. HRMS (DART+) for $\mathrm{C}_{23} \mathrm{H}_{39} \mathrm{BNO}_{5}[\mathrm{M}+\mathrm{H}]^{+}$: Calc'd: 420.2916 , found: 420.2938 .<smiles>CON(CCBr)CCC(Br)CBr</smiles>

tert-butyl

(3,4-bis(4,4,5,5-tetramethyl-1,3,2-dioxaborolan-2yl)butyl)(methoxy)carbamate (14) The reaction was performed according to General procedure $C$ with tert-butyl but-3-en-1-yl(methoxy)carbamate (20) (2.02 g, $10 \mathrm{mmol}, 1.0$ equiv.), bis(pinacolato)diboron ( $2.78 \mathrm{~g}, 11 \mathrm{mmol}, 1.1$ equiv.), cesium carbonate ( $0.49 \mathrm{~g}, 1.5 \mathrm{mmol}, 0.15$ equiv.), methanol (2.0 mL, $50 \mathrm{mmol}, 5.0$ equiv.) in THF $(30 \mathrm{~mL})$. The compound was purified with $\mathrm{SiO}_{2}$ chromatography (10\% ethyl acetate in hexane, stain with CAM) as a white solid (3.87 g, 85\%). ${ }^{1} \mathbf{H}$ NMR $\left(600 \mathrm{MHz}, \mathrm{CDCl}_{3}\right) \delta 3.63(\mathrm{~s}, 3 \mathrm{H}), 3.46-3.35(\mathrm{~m}, 2 \mathrm{H}), 1.80-1.70(\mathrm{~m}, 1 \mathrm{H})$, $1.62-1.53(\mathrm{~m}, 1 \mathrm{H}), 1.45(\mathrm{~s}, 9 \mathrm{H}), 1.20(\mathrm{~s}, 12 \mathrm{H}), 1.19(\mathrm{~s}, 12 \mathrm{H}), 1.13-1.07(\mathrm{~m}, 1 \mathrm{H}), 0.90-0.77$ $(\mathrm{m}, 2 \mathrm{H}) .{ }^{13} \mathrm{C} \mathrm{NMR}\left(151 \mathrm{MHz}, \mathrm{CDCl}_{3}\right) \delta 156.3,83.0,83.0,80.9,62.2,48.8,30.8,28.4,25.1,25.0$, 24.9, 24.9, 24.8, 15.9, 12.6. IR (neat) $v_{\max } 2976(\mathrm{~m}), 2931(\mathrm{w}), 1701(\mathrm{~m}), 1366(\mathrm{~s}), 1313(\mathrm{~s}), 1235$ (m), 1214 (w), 1143 (s), 1109 (w), 1087 (w), 967 (m), 860 (w), 846 (m), 765 (w), $671(\mathrm{w}), 578$ (w) $\mathrm{cm}^{-1}$. HRMS (DART+) for $\mathrm{C}_{22} \mathrm{H}_{44} \mathrm{~B}_{2} \mathrm{NO}_{7}[\mathrm{M}+\mathrm{H}]^{+}$: Calc'd: 456.3298, found: 456. 3300. Melting point: $71-74^{\circ} \mathrm{C}$.<smiles>CON(CCCC(Br)CBr)CC(=O)OCc1ccccc1</smiles>

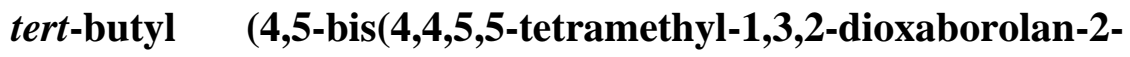
yl)pentyl)(methoxy)carbamate (16) The reaction was performed according to General procedure $C$ with tert-butyl methoxy(pent-4-en-1-yl)carbamate (25) (2.15 g, 10 mmol, 1.0 equiv.), bis(pinacolato)diboron $(2.78 \mathrm{~g}, 11 \mathrm{mmol}, 1.1$ equiv.), cesium carbonate $(0.49 \mathrm{~g}, 1.5 \mathrm{mmol}, 0.15$ equiv.), methanol (2.0 mL, $50 \mathrm{mmol}, 5.0$ equiv.) in THF (30 mL). The compound was purified with $\mathrm{SiO}_{2}$ chromatography (10\% ethyl acetate in hexane, stain with CAM) as a colorless oil (3.80 g, 81\%). ${ }^{1} \mathbf{H}$ NMR $\left(600 \mathrm{MHz}, \mathrm{CDCl}_{3}\right) \delta 3.61(\mathrm{~s}, 3 \mathrm{H}), 3.38-3.26(\mathrm{~m}, 2 \mathrm{H}), 1.61-1.52(\mathrm{~m}, 2 \mathrm{H})$, $1.46-1.37(\mathrm{~m}, 10 \mathrm{H}), 1.34-1.23(\mathrm{~m}, 1 \mathrm{H}), 1.17(\mathrm{~s}, 12 \mathrm{H}), 1.17(\mathrm{~s}, 12 \mathrm{H}), 1.12-1.04(\mathrm{~m}, 1 \mathrm{H}), 0.86$ - $0.72(\mathrm{~m}, 2 \mathrm{H}) .{ }^{13} \mathrm{C}$ NMR $\left(151 \mathrm{MHz}, \mathrm{CDCl}_{3}\right) \delta 156.4,82.9,80.9,62.3,49.7,30.9,28.4,26.5$, 25.1, 24.9, 24.9, 24.8, 24.8, 18.2, 12.7. IR (neat) $v_{\max } 2976(\mathrm{~m}), 2932(\mathrm{w}), 1702(\mathrm{~m}), 1458(\mathrm{w})$, 1366 (s), 1312 (s), 1274 (m), 1248 (m), 1214 (m), 1140 (s), 967 (m), 883 (w), 846 (m), 766 (w), $671(\mathrm{w}), 578(\mathrm{w}) \mathrm{cm}^{-1}$. HRMS (DART+) for $\mathrm{C}_{23} \mathrm{H}_{49} \mathrm{~B}_{2} \mathrm{~N}_{2} \mathrm{O}_{7}\left[\mathrm{M}+\mathrm{NH}_{4}\right]^{+}$: Calc'd: 487.3720, found: 487.3726. 


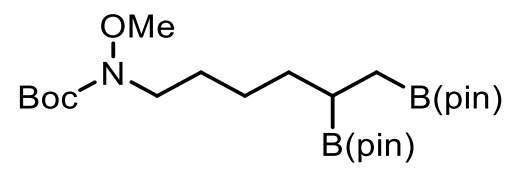

tert-butyl $\quad(5,6$-bis $(4,4,5,5$-tetramethyl-1,3,2-dioxaborolan-2yl)hexyl)(methoxy)carbamate (18) The reaction was performed according to General procedure $C$ with tert-butyl hex-5-en-1-yl(methoxy)carbamate (S-4) (2.29 g, $10 \mathrm{mmol}, 1.0$ equiv.), bis(pinacolato)diboron ( $2.78 \mathrm{~g}, 11 \mathrm{mmol}, 1.1$ equiv.), cesium carbonate $(0.49 \mathrm{~g}, 1.5 \mathrm{mmol}, 0.15$ equiv.), methanol ( $2.0 \mathrm{~mL}, 50 \mathrm{mmol}, 5.0$ equiv.) in THF ( $30 \mathrm{~mL})$. The compound was purified with $\mathrm{SiO}_{2}$ chromatography (10\% ethyl acetate in hexane, stain with CAM) as a colorless oil (3.2 g, 67\%). ${ }^{1} \mathbf{H}$ NMR $\left(600 \mathrm{MHz}, \mathrm{CDCl}_{3}\right) \delta 3.63(\mathrm{~s}, 3 \mathrm{H}), 3.39-3.32(\mathrm{~m}, 2 \mathrm{H}), 1.59-1.52(\mathrm{~m}, 2 \mathrm{H})$, $1.46(\mathrm{~s}, 10 \mathrm{H}), 1.33-1.25(\mathrm{~m}, 3 \mathrm{H}), 1.20(\mathrm{~s}, 24 \mathrm{H}), 1.13-1.04(\mathrm{~m}, 1 \mathrm{H}), 0.87-0.73(\mathrm{~m}, 2 \mathrm{H}) .{ }^{13} \mathrm{C}$ NMR $\left(151 \mathrm{MHz}, \mathrm{CDCl}_{3}\right) \delta 156.5,82.9,82.9,81.0,62.4,49.5,33.6,28.4,27.5,26.2,25.1,25.0$, 24.9, 24.9, 24.8, 18.5, 12.7. IR (neat) $v_{\max } 2976(\mathrm{w}), 2931(\mathrm{w}), 2864(\mathrm{w}), 1702(\mathrm{~m}), 1366(\mathrm{~m})$, $1311(\mathrm{~m}), 1269$ (w), 1214 (w), 1140 (s), 1109 (w), 994 (w), 967 (w), 859 (w), $846(\mathrm{~m}), 766$ (w), 735 (w), $703(w), 671(w), 577(w) \mathrm{cm}^{-1}$. HRMS (DART+) for $\mathrm{C}_{24} \mathrm{H}_{51} \mathrm{~B}_{2} \mathrm{~N}_{2} \mathrm{O}_{7}\left[\mathrm{M}+\mathrm{NH}_{4}\right]^{+}$: Calc'd: 501.3877, found: 501.3886 .<smiles>CON(CCCC(Br)C(C)Br)C(=O)OC(C)(C)C</smiles>

tert-butyl (rac-(4S,5S)-4,5-bis(4,4,5,5-tetramethyl-1,3,2-dioxaborolan2-yl)hexyl)(methoxy)carbamate (30) The reaction was performed according to General procedure $C$ with tert-butyl (E)-hex-4-en-1-yl(methoxy)carbamate $(29)(0.23 \mathrm{~g}, 1.0 \mathrm{mmol}, 1.0$ equiv.), bis(pinacolato)diboron ( $0.278 \mathrm{~g}, 11 \mathrm{mmol}, 1.1$ equiv.), cesium carbonate $(0.98 \mathrm{~g}, 0.3$ mmol, 0.15 equiv.), methanol $(0.2 \mathrm{~mL}, 5.0 \mathrm{mmol}, 5.0$ equiv. $)$ in THF $(1.5 \mathrm{~mL})$. The compound was purified with $\mathrm{SiO}_{2}$ chromatography (10\% ethyl acetate in hexane, stain with CAM) as a colorless oil $(0.20 \mathrm{~g}, 41 \%) .{ }^{1} \mathbf{H}$ NMR $\left(500 \mathrm{MHz}, \mathrm{CDCl}_{3}\right) \delta 3.62(\mathrm{~s}, 3 \mathrm{H}), 3.35(\mathrm{t}, J=7.0 \mathrm{~Hz}, 2 \mathrm{H})$, $1.66-1.55(\mathrm{~m}, 1 \mathrm{H}), 1.53-1.46(\mathrm{~m}, 2 \mathrm{H}), 1.44(\mathrm{~s}, 9 \mathrm{H}), 1.35-1.27(\mathrm{~m}, 1 \mathrm{H}), 1.21-1.15(\mathrm{~m}, 24 \mathrm{H})$, $1.14-1.09(\mathrm{~m}, 1 \mathrm{H}), 1.06-0.99(\mathrm{~m}, 1 \mathrm{H}), 0.92(\mathrm{~d}, J=7.5 \mathrm{~Hz}, 3 \mathrm{H}) .{ }^{\mathbf{1 3}} \mathbf{C} \mathbf{~ N M R}\left(126 \mathrm{MHz}, \mathrm{CDCl}_{3}\right)$ $\delta 156.4,82.9,82.8,80.9,62.3,49.9,28.4,27.0,26.8,26.0,25.1,25.0,24.8,24.7,17.9,14.5,13.9$. IR (neat) $v_{\max } 2976$ (w), 2932 (w), 1703 (w), 1459 (w), 1368 (s), 1311 (m), 1215 (w), 1142 (s), $969(\mathrm{w}), 858(\mathrm{w}) \mathrm{cm}^{-1}$. HRMS (DART+) for $\mathrm{C}_{24} \mathrm{H}_{51} \mathrm{~B}_{2} \mathrm{~N}_{2} \mathrm{O}_{7}\left[\mathrm{M}+\mathrm{NH}_{4}\right]^{+}$: Calc'd: 501.3877, found: 501.3897 .<smiles>C=CCCC(Br)CCN(OC)C(=O)OCc1ccccc1</smiles>

tert-butyl methoxy (3-(4,4,5,5-tetramethyl-1,3,2-dioxaborolan-2yl)hept-6-en-1-yl)carbamate (S-9) The reaction was performed using a method under development in the group from tert-butyl (3,4-bis(4,4,5,5-tetramethyl-1,3,2-dioxaborolan-2yl)butyl)(methoxy)carbamate (14) $(0.46 \mathrm{~g}, 1 \mathrm{mmol})$ and this method will be reported separately. 
The compound was purified with $\mathrm{SiO}_{2}$ chromatography (10\% ethyl acetate in hexane, stain with CAM) as a colorless oil (0.25 g, 68\%). ${ }^{1} \mathbf{H}$ NMR (600 MHz, $\left.\mathrm{CDCl}_{3}\right) \delta 5.84-5.73(\mathrm{~m}, 1 \mathrm{H}), 5.01$ $-4.95(\mathrm{~m}, 1 \mathrm{H}), 4.94-4.88(\mathrm{~m}, 1 \mathrm{H}), 3.65(\mathrm{~s}, 3 \mathrm{H}), 3.48-3.35(\mathrm{~m}, 2 \mathrm{H}), 2.13-1.98(\mathrm{~m}, 1 \mathrm{H}), 1.77$ $-1.69(\mathrm{~m}, 1 \mathrm{H}), 1.69-1.61(\mathrm{~m}, 1 \mathrm{H}), 1.59-1.50(\mathrm{~m}, 1 \mathrm{H}), 1.48(\mathrm{~s}, 10 \mathrm{H}), 1.23(\mathrm{~s}, 12 \mathrm{H}), 1.04-0.94$ $(\mathrm{m}, 1 \mathrm{H}) .{ }^{13} \mathrm{C}$ NMR $\left(151 \mathrm{MHz}, \mathrm{CDCl}_{3}\right) \delta 156.4,139.1,114.5,83.2,81.1,62.3,48.9,33.4,30.4$, 28.5, 28.4, 25.0, 24.9. IR (neat) $v_{\max } 2977(\mathrm{w}), 2931(\mathrm{w}), 1726(\mathrm{~m}), 1701(\mathrm{~m}), 1640(\mathrm{w}), 1457(\mathrm{w})$, 1380 (m), 1367 (m), 1318 (m), 1239 (m), 1160 (s), 1143 (s), 1085 (w), 995 (w), 967 (w), 909 (w), $859(\mathrm{w}), 765(\mathrm{w}), 693(\mathrm{w}), 670(\mathrm{w}), 578(\mathrm{w}) \mathrm{cm}^{-1}$. HRMS (DART+) for $\mathrm{C}_{19} \mathrm{H}_{37} \mathrm{BNO}_{5}[\mathrm{M}+\mathrm{H}]^{+}$: Calc'd: 370.2759 , found: 370.2763 .<smiles>C=CCCC(Br)CCCN(OC)C(=O)OCc1ccccc1</smiles>

tert-butyl methoxy(4-(4,4,5,5-tetramethyl-1,3,2-dioxaborolan-2yl)oct-7-en-1-yl)carbamate (S-10) The reaction was performed using a method under development in the group from tert-butyl (4,5-bis(4,4,5,5-tetramethyl-1,3,2-dioxaborolan-2yl)pentyl)(methoxy)carbamate (16) $(0.47 \mathrm{~g}, 1 \mathrm{mmol})$ and this method will be reported separately. The compound was purified with $\mathrm{SiO}_{2}$ chromatography (10\% ethyl acetate in hexane, stain with CAM) as a colorless oil $(0.24 \mathrm{~g}, 62 \%) .{ }^{1} \mathbf{H}$ NMR $\left(500 \mathrm{MHz}, \mathrm{CDCl}_{3}\right) \delta 5.83-5.69(\mathrm{~m}, 1 \mathrm{H}), 4.98$ $-4.91(\mathrm{~m}, 1 \mathrm{H}), 4.90-4.86(\mathrm{~m}, 1 \mathrm{H}), 3.63(\mathrm{~s}, 3 \mathrm{H}), 3.38-3.33(\mathrm{~m}, 2 \mathrm{H}), 2.08-1.95(\mathrm{~m}, 2 \mathrm{H}), 1.61$ $-1.53(\mathrm{~m}, 2 \mathrm{H}), 1.54-1.41(\mathrm{~m}, 10 \mathrm{H}), 1.43-1.28(\mathrm{~m}, 3 \mathrm{H}), 1.20(\mathrm{~s}, 12 \mathrm{H}), 1.02-0.91(\mathrm{~m}, 1 \mathrm{H}) .{ }^{13} \mathrm{C}$ NMR $\left(126 \mathrm{MHz}, \mathrm{CDCl}_{3}\right) \delta 156.4,139.2,114.4,83.0,81.0,62.3,49.5,33.4,30.6,28.4,26.8,24.9$, 23.2. IR (neat) $v_{\max } 2977(\mathrm{w}), 2931(\mathrm{w}), 2857(\mathrm{w}), 1725(\mathrm{w}), 1703(\mathrm{~m}), 1640(\mathrm{w}), 1458(\mathrm{w}), 1380$ (m), 1367 (s), 1316 (m), 1254 (w), 1214 (w), 1152 (s), 1144 (s), 1083 (w), 994 (w), 967 (w), 908 (w), $856(\mathrm{w}), 766(\mathrm{w}), 690(\mathrm{w}), 670(\mathrm{w}) \mathrm{cm}^{-1}$. HRMS (DART+) for $\mathrm{C}_{20} \mathrm{H}_{42} \mathrm{BN}_{2} \mathrm{O}_{5}\left[\mathrm{M}+\mathrm{NH}_{4}\right]^{+}$: Calc'd: 401.3181, found: 401.3196.

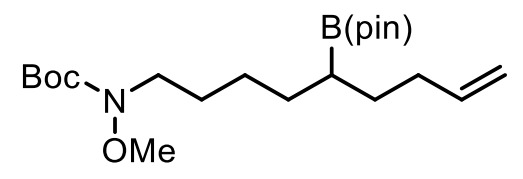

\section{tert-butyl methoxy $(5-(4,4,5,5$-tetramethyl-1,3,2-dioxaborolan-}

2-yl)non-8-en-1-yl)carbamate (S-11) The reaction was performed using a method under development in the group from tert-butyl (5,6-bis(4,4,5,5-tetramethyl-1,3,2-dioxaborolan-2yl)hexyl)(methoxy)carbamate (18) $(0.48 \mathrm{~g}, 1 \mathrm{mmol})$ and this method will be reported separately. The compound was purified with $\mathrm{SiO}_{2}$ chromatography (10\% ethyl acetate in hexane, stain with CAM) as a colorless oil $(0.26 \mathrm{~g}, 65 \%) .{ }^{1} \mathbf{H}$ NMR $\left(600 \mathrm{MHz}, \mathrm{CDCl}_{3}\right) \delta 5.82-5.73(\mathrm{~m}, 1 \mathrm{H}), 4.96$ $(\mathrm{dd}, J=17.1,1.9 \mathrm{~Hz}, 1 \mathrm{H}), 4.89$ (dd, $J=10.2,1.2 \mathrm{~Hz}, 1 \mathrm{H}), 3.64(\mathrm{~s}, 3 \mathrm{H}), 3.37$ (t, $J=7.3 \mathrm{~Hz}, 2 \mathrm{H})$, $2.08-1.95(\mathrm{~m}, 2 \mathrm{H}), 1.61-1.54(\mathrm{~m}, 2 \mathrm{H}), 1.53-1.37(\mathrm{~m}, 12 \mathrm{H}), 1.37-1.25(\mathrm{~m}, 3 \mathrm{H}), 1.21(\mathrm{~s}, 12 \mathrm{H})$, $1.00-0.92(\mathrm{~m}, 1 \mathrm{H}) .{ }^{13} \mathrm{C} \mathbf{N M R}\left(151 \mathrm{MHz}, \mathrm{CDCl}_{3}\right) \delta 156.5,139.3,114.3,83.0,81.1,62.4,49.3$, 33.5, 31.0, 30.7, 28.4, 27.6, 26.5, 24.9. IR (neat) $v_{\max } 2976(\mathrm{w}), 2930$ (w), 2856 (w), 1727 (w), 
1702 (m), 1640 (w), 1458 (w), 1387 (m), 1367 (m), 1315 (w), 1234 (w), 1143 (s), 994 (w), 967 (w), 908 (w), 859 (w), $766(w), 689$ (w), $670(w) \mathrm{cm}^{-1}$. HRMS (DART+) for $\mathrm{C}_{21} \mathrm{H}_{44} \mathrm{BN}_{2} \mathrm{O}_{5}$ $\left[\mathrm{M}+\mathrm{NH}_{4}\right]^{+}:$Calc'd: 415.3338, found: 415.3326 .

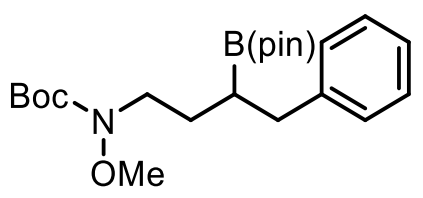

tert-butyl methoxy(4-phenyl-3-(4,4,5,5-tetramethyl-1,3,2-

dioxaborolan-2-yl)butyl)carbamate (S-12) The reaction was performed according to General procedure $\quad E$ with tert-butyl (3,4-bis(4,4,5,5-tetramethyl-1,3,2-dioxaborolan-2yl)butyl)(methoxy)carbamate (14) (0.46 g, $1 \mathrm{mmol}, 1.0$ equiv.), solid potassium hydroxide (0.17 g, $3 \mathrm{mmol}, 3$ equiv.), Pd(OAc) 2 (5.6 mg, $0.025 \mathrm{mmol}, 2.5 \mathrm{~mol} \%$ ), RuPhos (11.7 mg, $0.025 \mathrm{mmol}$, $2.5 \mathrm{~mol} \%)$ and bromobenzene ( $0.24 \mathrm{~g}, 1.5 \mathrm{mmol}, 1.5$ equiv.) in THF $(10 \mathrm{~mL})$ and $\mathrm{H}_{2} \mathrm{O}(1 \mathrm{~mL})$. The compound was purified with $\mathrm{SiO}_{2}$ chromatography (10\% ethyl acetate in hexane, stain with CAM) as a colorless oil $(0.24 \mathrm{~g}, 60 \%) .{ }^{1} \mathbf{H}$ NMR $\left(500 \mathrm{MHz}, \mathrm{CDCl}_{3}\right) \delta 7.24-7.17(\mathrm{~m}, 4 \mathrm{H}), 7.16$ $-7.10(\mathrm{~m}, 1 \mathrm{H}), 3.61(\mathrm{~s}, 3 \mathrm{H}), 3.50-3.37(\mathrm{~m}, 2 \mathrm{H}), 2.78-2.64(\mathrm{~m}, 2 \mathrm{H}), 1.79-1.63(\mathrm{~m}, 2 \mathrm{H}), 1.46$ (s, 9H), $1.39-1.32(\mathrm{~m}, 1 \mathrm{H}), 1.16(\mathrm{~s}, 6 \mathrm{H}), 1.14(\mathrm{~s}, 6 \mathrm{H}) .{ }^{\mathbf{1 3}} \mathbf{C} \mathbf{N M R}\left(151 \mathrm{MHz}, \mathrm{CDCl}_{3}\right) \delta 156.4$, 142.0, 129.0, 128.2, 125.8, 83.3, 81.1, 62.4, 48.9, 37.1, 28.5, 28.3, 24.9, 24.8. IR (neat) $v_{\max } 2976$ (w), $2931(\mathrm{w}), 1724(\mathrm{w}), 1700(\mathrm{~m}), 1454(\mathrm{w}), 1380(\mathrm{~m}), 1366(\mathrm{~s}), 1321(\mathrm{~m}), 1242(\mathrm{~m}), 1142(\mathrm{~s})$, 1111 (w), 967 (w), 880 (w), 747 (w), 699 (w) cm ${ }^{-1}$. HRMS (DART+) for $\mathrm{C}_{22} \mathrm{H}_{37} \mathrm{BNO}_{5}[\mathrm{M}+\mathrm{H}]^{+}$: Calc'd: 406.2759, found: 406.2761.<smiles>CON(CCC(Br)CC1=CCCC1)C(C)(C)C</smiles>

tert-butyl (4-(cyclopent-1-en-1-yl)-3-(4,4,5,5-tetramethyl-1,3,2dioxaborolan-2-yl)butyl)(methoxy)carbamate (S-13) The reaction was performed according to General procedure $E$ with tert-butyl (3,4-bis(4,4,5,5-tetramethyl-1,3,2-dioxaborolan-2yl)butyl)(methoxy)carbamate (14) (0.46 g, $1 \mathrm{mmol}, 1.0$ equiv.), solid potassium hydroxide (0.17 $\mathrm{g}, 3 \mathrm{mmol}, 3$ equiv.), $\mathrm{Pd}(\mathrm{OAc})_{2}(5.6 \mathrm{mg}, 0.025 \mathrm{mmol}, 2.5 \mathrm{~mol} \%)$, RuPhos (11.7 mg, $0.025 \mathrm{mmol}$, $2.5 \mathrm{~mol} \%)$ and 1-chlorocyclopentene $(0.15 \mathrm{~g}, 1.5 \mathrm{mmol}, 1.5$ equiv. $)$ in THF (10 mL) and $\mathrm{H}_{2} \mathrm{O}$ (1 $\mathrm{mL})$. The compound was purified with $\mathrm{SiO}_{2}$ chromatography (10\% ethyl acetate in hexane, stain with CAM) as a colorless oil (0.35 g, 89\%). ${ }^{1} \mathbf{H} \mathbf{~ N M R}\left(600 \mathrm{MHz}, \mathrm{CDCl}_{3}\right) \delta 5.35(\mathrm{~s}, 1 \mathrm{H}), 3.66(\mathrm{~s}$, $3 \mathrm{H}), 3.46-3.37(\mathrm{~m}, 2 \mathrm{H}), 2.28-2.17(\mathrm{~m}, 5 \mathrm{H}), 2.17-2.11(\mathrm{~m}, 1 \mathrm{H}), 1.86-1.79(\mathrm{~m}, 2 \mathrm{H}), 1.75-$ $1.61(\mathrm{~m}, 2 \mathrm{H}), 1.48(\mathrm{~s}, 9 \mathrm{H}), 1.26-1.17(\mathrm{~m}, 13 \mathrm{H}) .{ }^{13} \mathrm{C} \mathrm{NMR}\left(151 \mathrm{MHz}, \mathrm{CDCl}_{3}\right) \delta 156.4,144.4$, 124.3, 83.2, 81.1, 62.3, 49.0, 35.2, 32.6, 32.5, 28.6, 28.5, 25.0, 24.9, 23.7. IR (neat) $v_{\max } 2976(\mathrm{w})$, 2931 (w), 2843 (w), 1725 (w), 1701 (m), 1438 (w), 1379 (m), 1366 (m), 1321 (m), 1241 (m), 1142 (s), 1091 (w), 1061 (w), 1034 (w), 967 (w), 860 (w), 835 (w), 766 (w), 669 (w) cm cm $^{-1}$ HRMS (DART+) for $\mathrm{C}_{21} \mathrm{H}_{39} \mathrm{BNO}_{5}[\mathrm{M}+\mathrm{H}]^{+}$: Calc'd: 396.2916, found: 396.2913. 
<smiles>CON(CCC(Br)Cc1ccoc1)C(=O)OCc1ccccc1</smiles>

tert-butyl (4-(furan-3-yl)-3-(4,4,5,5-tetramethyl-1,3,2-dioxaborolan-2yl)butyl)(methoxy)carbamate (S-14) The reaction was performed according to General procedure $\quad E \quad$ with tert-butyl (3,4-bis(4,4,5,5-tetramethyl-1,3,2-dioxaborolan-2yl)butyl)(methoxy)carbamate (14) (0.46 g, $1 \mathrm{mmol}, 1.0$ equiv.), solid potassium hydroxide $(0.17$ $\mathrm{g}, 3 \mathrm{mmol}, 3$ equiv.), Pd(OAc) 2 (5.6 mg, $0.025 \mathrm{mmol}, 2.5 \mathrm{~mol} \%)$, RuPhos (11.7 mg, $0.025 \mathrm{mmol}$, $2.5 \mathrm{~mol} \%$ ), 3-bromofuran ( $0.22 \mathrm{~g}, 1.5 \mathrm{mmol}, 1.5$ equiv.) and $\mathrm{LiCl}$ (42 mg, $1.0 \mathrm{mmol}, 1.0$ equiv.) in THF $(10 \mathrm{~mL})$ and $\mathrm{H}_{2} \mathrm{O}(1 \mathrm{~mL})$. The compound was purified with $\mathrm{SiO}_{2}$ chromatography $(10 \%$ ethyl acetate in hexane, stain with CAM) as a colorless oil $(0.20 \mathrm{~g}, 50 \%) .{ }^{1} \mathbf{H}$ NMR $(600 \mathrm{MHz}$, $\left.\mathrm{CDCl}_{3}\right) \delta 7.26(\mathrm{~s}, 1 \mathrm{H}), 7.18(\mathrm{~s}, 1 \mathrm{H}), 6.24(\mathrm{~s}, 1 \mathrm{H}), 3.60(\mathrm{~s}, 3 \mathrm{H}), 3.46-3.35(\mathrm{~m}, 2 \mathrm{H}), 2.57-2.41$ $(\mathrm{m}, 2 \mathrm{H}), 1.76-1.61(\mathrm{~m}, 2 \mathrm{H}), 1.44(\mathrm{~s}, 9 \mathrm{H}), 1.26-1.18(\mathrm{~m}, 1 \mathrm{H}), 1.14(\mathrm{~s}, 12 \mathrm{H}) .{ }^{13} \mathrm{C}$ NMR $(151$ $\left.\mathrm{MHz}, \mathrm{CDCl}_{3}\right) \delta 156.3,142.5,139.4,124.4,111.4,83.2,81.0,62.2,48.7,28.4,28.2,25.9,24.8$, 24.8. IR (neat) $v_{\max } 2976(\mathrm{w}), 2931(\mathrm{w}), 1698(\mathrm{~m}), 1456(\mathrm{w}), 1366(\mathrm{~m}), 1319(\mathrm{~m}), 1241(\mathrm{~m}), 1151$ (m), 1141 (s), 1093 (w), 1063 (w), 1023 (m), 967 (w), 872 (m), 860 (w), 774 (w), 737 (w), 670 (w), $599(\mathrm{~m}) \mathrm{cm}^{-1}$. HRMS (DART+) for $\mathrm{C}_{20} \mathrm{H}_{35} \mathrm{BNO}_{6}[\mathrm{M}+\mathrm{H}]^{+}$: Calc'd: 396.2552, found: 396.2550 .<smiles>CON(CCC(Br)CCCCBr)C(=O)OCc1ccccc1</smiles>

tert-butyl (3,7-bis $(4,4,5,5$-tetramethyl-1,3,2-dioxaborolan-2-

yl)heptyl)(methoxy)carbamate (S-15) The reaction was performed according to General procedure $D$ with tert-butyl methoxy(3-(4,4,5,5-tetramethyl-1,3,2-dioxaborolan-2-yl)hept-6-en-1yl)carbamate (S-9) (0.37 g, $1.0 \mathrm{mmol}, 1.0$ equiv.), [ $\operatorname{Ir}(\mathrm{cod}) \mathrm{Cl}]_{2}(10.1 \mathrm{mg}, 0.015 \mathrm{mmol}, 1.5 \mathrm{~mol} \%)$, bis(diphenylphosphino)methane (11.5 mg, $0.03 \mathrm{mmol}, 3 \mathrm{~mol} \%)$, pinacol borane $(0.17 \mathrm{~mL}, 1.2$ mmol, 1.2 equiv.) in DCM (4 mL). The compound was purified with $\mathrm{SiO}_{2}$ chromatography (15\% ethyl acetate in hexane, stain with CAM) as a colorless oil $(0.42 \mathrm{~g}, 85 \%) .{ }^{1} \mathbf{H} \mathbf{~ N M R}(500 \mathrm{MHz}$, $\left.\mathrm{CDCl}_{3}\right) \delta 3.63(\mathrm{~s}, 3 \mathrm{H}), 3.43-3.31(\mathrm{~m}, 2 \mathrm{H}), 1.73-1.56(\mathrm{~m}, 2 \mathrm{H}), 1.45(\mathrm{~s}, 9 \mathrm{H}), 1.42-1.30(\mathrm{~m}, 4 \mathrm{H})$, $1.30-1.23(\mathrm{~m}, 2 \mathrm{H}), 1.20(\mathrm{~s}, 24 \mathrm{H}), 0.96-0.89(\mathrm{~m}, 1 \mathrm{H}), 0.75-0.69(\mathrm{~m}, 2 \mathrm{H}) .{ }^{13} \mathbf{C ~ N M R}(126 \mathrm{MHz}$, $\left.\mathrm{CDCl}_{3}\right) \delta 156.4,83.0,82.9,81.0,62.3,49.1,32.0,31.0,28.5,28.4,24.9,24.9,24.9,24.4$. IR (neat) $v_{\max } 2976(\mathrm{w}), 2929(\mathrm{w}), 2860(\mathrm{w}), 1742(\mathrm{w}), 1701(\mathrm{~m}), 1459(\mathrm{w}), 1367(\mathrm{~s}), 1315(\mathrm{~s}), 1242(\mathrm{~m})$, 1414 (m), 1144 (s), 1111 (m), 1089 (w), 1031 (w), 967 (m), 859 (w), 846 (m), 766 (w), 671 (w), $578(\mathrm{w}) \mathrm{cm}^{-1}$. HRMS (DART+) for $\mathrm{C}_{25} \mathrm{H}_{50} \mathrm{~B}_{2} \mathrm{NO}_{7}[\mathrm{M}+\mathrm{H}]^{+}$: Calc'd: 498.3768, found: 498.3785. 


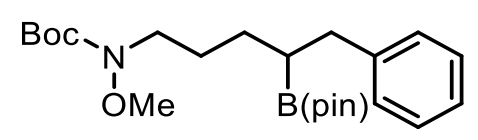

tert-butyl

methoxy (5-phenyl-4-(4,4,5,5-tetramethyl-1,3,2-

dioxaborolan-2-yl)pentyl)carbamate (S-16) The reaction was performed according to General procedure $E$ with tert-butyl (4,5-bis(4,4,5,5-tetramethyl-1,3,2-dioxaborolan-2yl)pentyl)(methoxy)carbamate (16) ( $0.47 \mathrm{~g}, 1 \mathrm{mmol}, 1.0$ equiv.), solid potassium hydroxide (0.17 g, $3 \mathrm{mmol}, 3$ equiv.), $\mathrm{Pd}(\mathrm{OAc})_{2}(5.6 \mathrm{mg}, 0.025 \mathrm{mmol}, 2.5 \mathrm{~mol} \%)$, RuPhos (11.7 mg, $0.025 \mathrm{mmol}$, $2.5 \mathrm{~mol} \%)$ and bromobenzene $(0.24 \mathrm{~g}, 1.5 \mathrm{mmol}, 1.5$ equiv. $)$ in THF $(10 \mathrm{~mL})$ and $\mathrm{H}_{2} \mathrm{O}(1 \mathrm{~mL})$. The compound was purified with $\mathrm{SiO}_{2}$ chromatography (10\% ethyl acetate in hexane, stain with CAM) as a colorless oil $(0.25 \mathrm{~g}, 60 \%) .{ }^{1} \mathbf{H} \mathbf{~ N M R}\left(500 \mathrm{MHz}, \mathrm{CDCl}_{3}\right) \delta 7.24-7.16(\mathrm{~m}, 4 \mathrm{H}), 7.15$ $-7.10(\mathrm{~m}, 1 \mathrm{H}), 3.63(\mathrm{~s}, 3 \mathrm{H}), 3.37(\mathrm{t}, J=7.3,2 \mathrm{H}), 2.75-2.61(\mathrm{~m}, 2 \mathrm{H}), 1.69-1.55(\mathrm{~m}, 2 \mathrm{H}), 1.47$ (s, 9H), $1.45-1.33(\mathrm{~m}, 3 \mathrm{H}), 1.15(\mathrm{~s}, 6 \mathrm{H}), 1.12(\mathrm{~s}, 6 \mathrm{H}) .{ }^{13} \mathrm{C} \mathbf{~ N M R}\left(151 \mathrm{MHz}, \mathrm{CDCl}_{3}\right) \delta 156.5$, $142.2,129.0,128.2,125.8,83.2,81.1,62.3,49.6,37.4,28.5,28.4,26.8,24.9,24.9$. IR (neat) $v_{\max }$ 2976 (w), 2931 (w), 2857 (w), $1701(\mathrm{~m}), 1603$ (w), 1454 (w), 1380 (m), 1367 (m), $1320(\mathrm{~m}), 1270$ (w), 1250 (w), 1141 (s), 1112 (w), 1033 (w), 989 (w), 968 (w), 857 (w), 748 (w), 699 (w), 579 (w) $\mathrm{cm}^{-1}$. HRMS (DART+) for $\mathrm{C}_{23} \mathrm{H}_{42} \mathrm{BN}_{2} \mathrm{O}_{5}\left[\mathrm{M}+\mathrm{NH}_{4}\right]^{+}$: Calc'd: 437.3181, found: 437.3176.<smiles>COc1ccc(CC(Br)CCCN(C)C(C)(C)C)cc1</smiles>

tert-butyl methoxy(5-(4-methoxyphenyl)-4-(4,4,5,5tetramethyl-1,3,2-dioxaborolan-2-yl)pentyl)carbamate (S-17) The reaction was performed according to General procedure E with tert-butyl (4,5-bis(4,4,5,5-tetramethyl-1,3,2-dioxaborolan2-yl)pentyl)(methoxy)carbamate (16) $(0.47 \mathrm{~g}, 1 \mathrm{mmol}, 1.0$ equiv.), solid potassium hydroxide (0.17 g, 3 mmol, 3 equiv.), Pd(OAc) 2 (5.6 mg, $0.025 \mathrm{mmol}, 2.5 \mathrm{~mol} \%$ ), RuPhos (11.7 mg, 0.025 $\mathrm{mmol}, 2.5 \mathrm{~mol} \%)$ and 1-bromo-4-methoxybenzene ( $0.28 \mathrm{~g}, 1.5 \mathrm{mmol}, 1.5$ equiv. $)$ in THF (10 mL) and $\mathrm{H}_{2} \mathrm{O}(1 \mathrm{~mL})$. The compound was purified with $\mathrm{SiO}_{2}$ chromatography (12\% ethyl acetate in hexane, stain with CAM) as a colorless oil $(0.35 \mathrm{~g}, 79 \%) .{ }^{1} \mathbf{H} \mathbf{~ N M R}\left(500 \mathrm{MHz}, \mathrm{CDCl}_{3}\right) \delta 7.09$ (d, $J=8.2 \mathrm{~Hz}, 2 \mathrm{H}), 6.77(\mathrm{~d}, J=8.2 \mathrm{~Hz}, 2 \mathrm{H}), 3.75(\mathrm{~s}, 3 \mathrm{H}), 3.63(\mathrm{~s}, 3 \mathrm{H}), 3.37$ (t, $J=7.3,2 \mathrm{H}), 2.70-$ $2.55(\mathrm{~m}, 2 \mathrm{H}), 1.68-1.54(\mathrm{~m}, 2 \mathrm{H}), 1.47(\mathrm{~s}, 9 \mathrm{H}), 1.43-1.28(\mathrm{~m}, 3 \mathrm{H}), 1.15(\mathrm{~s}, 6 \mathrm{H}), 1.13(\mathrm{~s}, 6 \mathrm{H})$. ${ }^{13} \mathbf{C}$ NMR $\left(126 \mathrm{MHz}, \mathrm{CDCl}_{3}\right) \delta 157.8,156.4,134.3,129.8,113.6,83.1,81.0,62.3,55.3,49.5$, 36.4, 28.4, 28.3, 26.8, 26.0, 24.9, 24.8. IR (neat) $v_{\max } 2976(\mathrm{w}), 2931$ (w), $2857(\mathrm{w}), 1700(\mathrm{~m})$, $1611(\mathrm{w}), 1511(\mathrm{~m}), 1458(\mathrm{w}), 1366(\mathrm{~s}), 1319(\mathrm{~m}), 1299(\mathrm{~m}), 1245(\mathrm{~s}), 1140(\mathrm{~s}), 1112(\mathrm{~m}), 1061$ (w), $1036(\mathrm{~m}), 967$ (w), $854(\mathrm{~m}), 839(\mathrm{~m}), 762(\mathrm{w}), 686(\mathrm{w}), 670(\mathrm{w}) \mathrm{cm}^{-1}$. HRMS (DART+) for $\mathrm{C}_{24} \mathrm{H}_{44} \mathrm{BN}_{2} \mathrm{O}_{6}\left[\mathrm{M}+\mathrm{NH}_{4}\right]^{+}$: Calc'd: 467.3287, found: 467.3293 .<smiles>CON(CCCC(CBr)Cc1ccccc1)C(=O)OCc1ccccc1</smiles>

tert-butyl (4-benzyl-5-(4,4,5,5-tetramethyl-1,3,2-dioxaborolan-2yl)pentyl)(methoxy)carbamate (S-18) In an oven-dried $20 \mathrm{~mL}$ vial, tert-butyl methoxy(5- 
phenyl-4-(4,4,5,5-tetramethyl-1,3,2-dioxaborolan-2-yl)pentyl)carbamate (S-16) (0.42 g, 1.0 mmol, 1.0 equiv.) and bromochloromethane $(0.20 \mathrm{~mL}, 3.0 \mathrm{mmol}, 3.0$ equiv.) were dissolved in anhydrous THF (7.5 mL). After the solution was cooled to $-78^{\circ} \mathrm{C}, n$-butyllithium in hexane (1.6 M, $1.56 \mathrm{~mL}, 2.5 \mathrm{mmol}, 2.5$ equiv.) was added dropwise, and the reaction mixture was stirred at this temperature for 20 mins before the cooling bath was removed. The mixture was allowed to warm up to r.t. and stirred for $2 \mathrm{~h}$. The reaction mixture was quenched by the addition of saturated ammonium chloride solution, extracted with ethyl acetate $(3 \times 20 \mathrm{~mL})$, dried over anhydrous sodium sulfate, and the solvent was removed under reduced pressure. compound was purified with $\mathrm{SiO}_{2}$ chromatography (10\% ethyl acetate in hexane, stain with CAM) as a colorless oil (0.35 g, $81 \%)$. The isolated compound contained $9 \%$ of unreacted starting material $(\mathbf{S}-\mathbf{2 3})$ and was used without further purification. ${ }^{1} \mathbf{H}$ NMR $\left(400 \mathrm{MHz}, \mathrm{CDCl}_{3}\right) \delta 7.26-7.19(\mathrm{~m}, 2 \mathrm{H}), 7.18-7.11(\mathrm{~m}$, $3 \mathrm{H}), 3.62(\mathrm{~s}, 3 \mathrm{H}), 3.33(\mathrm{t}, J=7.3 \mathrm{~Hz}, 2 \mathrm{H}), 2.55(\mathrm{~d}, J=7.7 \mathrm{~Hz}, 2 \mathrm{H}), 1.96-1.85(\mathrm{~m}, 1 \mathrm{H}), 1.72-$ $1.51(\mathrm{~m}, 2 \mathrm{H}), 1.46(\mathrm{~s}, 9 \mathrm{H}), 1.38-1.26(\mathrm{~m}, 1 \mathrm{H}), 1.21(\mathrm{~s}, 12 \mathrm{H}), 0.76(\mathrm{~d}, J=6.0 \mathrm{~Hz}, 2 \mathrm{H}) .{ }^{\mathbf{1 3}} \mathbf{C} \mathbf{~ N M R}$ $\left(151 \mathrm{MHz}, \mathrm{CDCl}_{3}\right) \delta 156.5,141.5,129.5,128.2,128.2,125.7,83.0,81.1,62.4,49.6,43.1,36.4$, 33.1, 28.5, 25.0, 24.7. IR (neat) $v_{\max } 2976(\mathrm{w}), 2930(\mathrm{w}), 1701(\mathrm{~m}), 1454(\mathrm{w}), 1366(\mathrm{~s}), 1317(\mathrm{~m})$, 1248 (m), 1142 (s), 1113 (m), 1032 (w), 968 (m), 847 (m), 740 (m), 700 (m), 577 (w) cm c $^{-1}$ HRMS (DART+) for $\mathrm{C}_{24} \mathrm{H}_{41} \mathrm{BNO}_{5}[\mathrm{M}+\mathrm{H}]^{+}$: Calc'd: 434.3072, found: 434.3079.<smiles>CON(CCCCC(Br)Cc1ccccc1Br)C(C)(C)C</smiles>

tert-butyl methoxy(6-phenyl-5-(4,4,5,5-tetramethyl-1,3,2dioxaborolan-2-yl)hexyl)carbamate (S-19) The reaction was performed according to General procedure $\quad E$ with tert-butyl (5,6-bis(4,4,5,5-tetramethyl-1,3,2-dioxaborolan-2yl)hexyl)(methoxy)carbamate (18) (0.48 g, $1.0 \mathrm{mmol}, 1.0$ equiv.), solid potassium hydroxide (0.17 $\mathrm{g}, 3 \mathrm{mmol}, 3$ equiv.), $\mathrm{Pd}(\mathrm{OAc})_{2}(5.6 \mathrm{mg}, 0.025 \mathrm{mmol}, 2.5 \mathrm{~mol} \%)$, RuPhos (11.7 mg, $0.025 \mathrm{mmol}$, $2.5 \mathrm{~mol} \%)$ and bromobenzene $(0.24 \mathrm{~g}, 1.5 \mathrm{mmol}, 1.5$ equiv. $)$ in THF $(10 \mathrm{~mL})$ and $\mathrm{H}_{2} \mathrm{O}(1 \mathrm{~mL})$. The compound was purified with $\mathrm{SiO}_{2}$ chromatography (10\% ethyl acetate in hexane, stain with CAM) as a colorless oil $(0.34 \mathrm{~g}, 79 \%) .{ }^{1} \mathbf{H} \mathbf{~ N M R}\left(600 \mathrm{MHz}, \mathrm{CDCl}_{3}\right) \delta 7.26-7.16(\mathrm{~m}, 4 \mathrm{H}), 7.16$ $-7.10(\mathrm{~m}, 1 \mathrm{H}), 3.65(\mathrm{~s}, 3 \mathrm{H}), 3.41-3.35(\mathrm{~m}, 2 \mathrm{H}), 2.74-2.62(\mathrm{~m}, 2 \mathrm{H}), 1.63-1.54(\mathrm{~m}, 2 \mathrm{H}), 1.48$ $(\mathrm{s}, 9 \mathrm{H}), 1.46-1.30(\mathrm{~m}, 5 \mathrm{H}), 1.15(\mathrm{~s}, 6 \mathrm{H}), 1.12(\mathrm{~s}, 6 \mathrm{H}) .{ }^{13} \mathbf{C ~ N M R}\left(151 \mathrm{MHz}, \mathrm{CDCl}_{3}\right) \delta 156.5$, 142.3, 129.0, 128.2, 125.7, 83.1, 81.1, 62.4, 49.3, 37.4, 31.0, 28.5, 27.6, 26.5, 24.9, 24.8. IR (neat) $v_{\max } 2976(\mathrm{w}), 2930(\mathrm{w}), 2856(\mathrm{w}), 1723(\mathrm{w}), 1701(\mathrm{~m}), 1454(\mathrm{w}), 1380(\mathrm{~m}), 1366(\mathrm{~m}), 1319(\mathrm{~m})$, 1252 (w), 1239 (w), 1214 (w), 1142 (s), $1111(\mathrm{w}), 1074$ (w), 1031 (w), 966 (w), 861 (m), 749 (w), 699 (m), 670 (w), 578 (w) cm ${ }^{-1}$. HRMS (DART+) for $\mathrm{C}_{24} \mathrm{H}_{44} \mathrm{BN}_{2} \mathrm{O}_{5}\left[\mathrm{M}+\mathrm{NH}_{4}\right]^{+}$: Calc'd: 451.3338 , found: 451.3351 . 


\section{Representative Procedure of Intramolecular Amination}

\section{Intramolecular amination followed by Boc protection (Method A):}

The corresponding tert-butyl(methoxy)carbamate substrate $(0.2 \mathrm{mmol})$ was dissolved in dichloromethane $(2 \mathrm{~mL})$. Trifluoroacetic acid $(2.0 \mathrm{mmol}, 0.15 \mathrm{~mL})$ was added dropwise. The mixture was allowed to stir at r.t. for $90 \mathrm{~min}$ and monitored by TLC. The mixture was quenched carefully with saturated sodium bicarbonate solution, extracted with ethyl acetate, and dried over anhydrous sodium sulfate and concentrated. The product was used without further purification.

The deprotected methoxy amine substrate was transferred into an oven-dried $20 \mathrm{~mL}$ vial loaded with a stir bar, and brought into the glovebox. Potassium tert-butoxide $(0.22 \mathrm{mmol}, 24.5 \mathrm{mg})$ was added, followed by toluene $(3.6 \mathrm{~mL})$ and THF $(0.36 \mathrm{~mL})$. The vial was sealed with a PTFE screwcap, removed from the glovebox, and heated at $110{ }^{\circ} \mathrm{C}$ (reaction time: 24 hours for azetidines, and 14 hours for pyrrolidines and piperidines). After the reaction was cooled to r.t., a solution of ditert-butyl dicarbonate $(0.3 \mathrm{mmol}, 35 \mathrm{mg})$ in THF $(1.5 \mathrm{~mL})$ was added and the mixture was allowed to stir for 3 hours. The mixture was washed with water, extracted with ethyl acetate, dried over anhydrous sodium sulfate and concentrated. The crude mixture was then purified using $\mathrm{SiO}_{2}$ chromatography.

\section{Intramolecular amination followed by Ts protection (Method B):}

The corresponding tert-butyl(methoxy)carbamate substrate $(0.2 \mathrm{mmol})$ was dissolved in dichloromethane $(2 \mathrm{~mL})$. Trifluoroacetic acid $(2.0 \mathrm{mmol}, 0.15 \mathrm{~mL})$ was added dropwise. The mixture was allowed to stir at r.t. for $90 \mathrm{~min}$ and monitored by TLC. The mixture was quenched carefully with saturated sodium bicarbonate solution, extracted with ethyl acetate, and dried over anhydrous sodium sulfate and concentrated. The product was used without further purification.

The deprotected methoxy amine substrate was transferred into an oven-dried $20 \mathrm{~mL}$ vial loaded with a stir bar, and brought into the glovebox. Potassium tert-butoxide $(0.22 \mathrm{mmol}, 24.5 \mathrm{mg})$ was added, followed by toluene $(3.6 \mathrm{~mL})$ and THF $(0.36 \mathrm{~mL})$. The vial was sealed with a PTFE screwcap, removed from the glovebox, and heated at $110^{\circ} \mathrm{C}$ (reaction time: 24 hours for azetidines, and 14 hours for pyrrolidines and piperidines). After the reaction was cooled to r.t., a solution of tosyl chloride $(0.4 \mathrm{mmol}, 28 \mathrm{mg})$ in THF $(2 \mathrm{~mL})$ was added, followed by triethylamine $(0.5 \mathrm{mmol}, 0.07$ $\mathrm{mL}$ ) and the mixture was allowed to stir for 3 hours. The mixture was washed with water, extracted with ethyl acetate, dried over anhydrous sodium sulfate and concentrated. The crude mixture was then purified using $\mathrm{SiO}_{2}$ chromatography. 


\section{Procedures and Characterization for Intramolecular Amination Product}

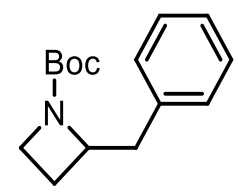

tert-butyl 2-benzylazetidine-1-carboxylate (1) The reaction was performed according to the general procedure above (Method $\boldsymbol{A}$ ) with tert-butyl methoxy(4-phenyl-3(4,4,5,5-tetramethyl-1,3,2-dioxaborolan-2-yl)butyl)carbamate (S-12) (83.9 mg, $0.20 \mathrm{mmol}, 1.0$ equiv.), and potassium tert-butoxide $(24.7 \mathrm{mg}, 0.22 \mathrm{mmol}, 1.1$ equiv.) in toluene and THF (3.6/0.36 mL, $0.05 \mathrm{M}$ ) for 24 hours. The compound was purified with $\mathrm{SiO}_{2}$ chromatography (10\% ethyl acetate in hexane, stain with Ninhydrin) as a colorless oil $(33.6 \mathrm{mg}, 68 \%)$. All spectral data was in accordance with previously published results. ${ }^{10}$

tert-butyl 2-(but-3-en-1-yl)azetidine-1-carboxylate (2) The reaction was performed according to the general procedure above (Method $\boldsymbol{A})$ with tert-butyl methoxy(3(4,4,5,5-tetramethyl-1,3,2-dioxaborolan-2-yl)hept-6-en-1-yl)carbamate $(\mathbf{S - 9}) \quad(73.9 \mathrm{mg}, 0.20$ mmol, 1.0 equiv.), and potassium tert-butoxide (24.7 $\mathrm{mg}, 0.22 \mathrm{mmol}, 1.1$ equiv.) in toluene and THF (3.6/0.36 mL, 0.05 M) for 24 hours. The compound was purified with $\mathrm{SiO}_{2}$ chromatography ( $10 \%$ ethyl acetate in hexane, stain with $\left.\mathrm{KMnO}_{4}\right)$ as a colorless oil (19.8 mg, 47\%). ${ }^{1} \mathbf{H}$ NMR (500 $\left.\mathrm{MHz}, \mathrm{CDCl}_{3}\right) \delta 5.90-5.74(\mathrm{~m}, 1 \mathrm{H}), 5.02(\mathrm{~d}, J=17.1 \mathrm{~Hz}, 1 \mathrm{H}), 4.95(\mathrm{~d}, J=10.3 \mathrm{~Hz}, 1 \mathrm{H}), 4.26-$ $4.15(\mathrm{~m}, 1 \mathrm{H}), 3.88-3.73(\mathrm{~m}, 2 \mathrm{H}), 2.29-2.19(\mathrm{~m}, 1 \mathrm{H}), 2.13-2.04(\mathrm{~m}, 2 \mathrm{H}), 2.03-1.93(\mathrm{~m}, 1 \mathrm{H})$, $1.88-1.78(\mathrm{~m}, 1 \mathrm{H}), 1.74-1.64(\mathrm{~m}, 1 \mathrm{H}), 1.43(\mathrm{~s}, 9 \mathrm{H}) .{ }^{13} \mathbf{C} \mathbf{N M R}\left(126 \mathrm{MHz}, \mathrm{CDCl}_{3}\right) \delta 156.8$, 138.3, 114.7, 79.2, 61.8, 46.3, 34.8, 30.4, 29.1, 28.6, 22.1. IR (neat) $v_{\max } 2928$ (w), 2891 (w), 2861

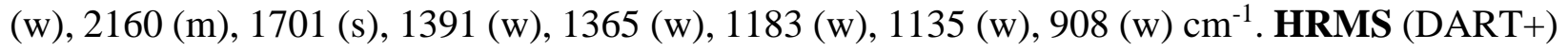
for $\mathrm{C}_{12} \mathrm{H}_{22} \mathrm{NO}_{2}[\mathrm{M}+\mathrm{H}]^{+}$: Calc'd: 212.1645, found: 212.1643 .

tert-butyl 2-(cyclopent-1-en-1-ylmethyl)azetidine-1-carboxylate (3) The reaction was performed according to the general procedure above (Method A) with tert-butyl (4(cyclopent-1-en-1-yl)-3-(4,4,5,5-tetramethyl-1,3,2-dioxaborolan-2-yl)butyl)(methoxy)carbamate (S-13) (79.0 mg, $0.20 \mathrm{mmol}, 1.0$ equiv.), and potassium tert-butoxide (24.7 $\mathrm{mg}, 0.22 \mathrm{mmol}, 1.1$ equiv.) in toluene and THF (3.6/0.36 mL, $0.05 \mathrm{M})$ for 24 hours. The compound was purified with $\mathrm{SiO}_{2}$ chromatography (10\% ethyl acetate in hexane, stain with $\mathrm{KMnO}_{4}$ ) as a colorless oil (28.5 mg, 60\%). ${ }^{1} \mathbf{H}$ NMR (500 MHz, $\left.\mathrm{CDCl}_{3}\right) \delta 5.32(\mathrm{~s}, 1 \mathrm{H}), 4.34-4.24(\mathrm{~m}, 1 \mathrm{H}), 3.85-3.72(\mathrm{~m}, 2 \mathrm{H})$, $2.72(\mathrm{~d}, J=14.6 \mathrm{~Hz}, 1 \mathrm{H}), 2.36(\mathrm{dd}, J=14.0,9.3 \mathrm{~Hz}, 1 \mathrm{H}), 2.31-2.25(\mathrm{~m}, 2 \mathrm{H}), 2.25-2.19$ (m, $3 \mathrm{H}), 1.89-1.79(\mathrm{~m}, 3 \mathrm{H}), 1.43(\mathrm{~s}, 9 \mathrm{H}) .{ }^{13} \mathbf{C} \mathbf{N M R}\left(126 \mathrm{MHz}, \mathrm{CDCl}_{3}\right) \delta 156.5,140.2,125.7,79.2$, 
60.9, 37.0, 35.9, 32.6, 28.6, 23.5, 22.4. IR (neat) $v_{\max } 2929$ (w), 2890 (w), 2845 (w), 1698 (s), 1478 (w), 1455 (w), 1388 (s), 1363 (s), 1254 (w), 1181 (w), 1131 (s), 1082 (w), 864 (w), 777 (w), 564 (w) $\mathrm{cm}^{-1}$. HRMS (DART+) for $\mathrm{C}_{14} \mathrm{H}_{24} \mathrm{NO}_{2}[\mathrm{M}+\mathrm{H}]^{+}$: Calc'd: 238.1802, found: 238.1815.

tert-butyl 2-(furan-3-ylmethyl)azetidine-1-carboxylate (4) The reaction was performed according to the general procedure above (Method A) with tert-butyl (4-(furan-3-yl)3-(4,4,5,5-tetramethyl-1,3,2-dioxaborolan-2-yl)butyl)(methoxy)carbamate (S-14) (79.1 mg, 0.20 mmol, 1.0 equiv.), and potassium tert-butoxide (24.7 $\mathrm{mg}, 0.22 \mathrm{mmol}, 1.1$ equiv.) in toluene and THF (3.6/0.36 mL, $0.05 \mathrm{M})$ for 24 hours. The compound was purified with $\mathrm{SiO}_{2}$ chromatography (10\% ethyl acetate in hexane, stain with CAM) as a colorless oil (31.0 mg, 66\%). ${ }^{\mathbf{1}} \mathbf{H}$ NMR (600 $\left.\mathrm{MHz} \mathrm{CDCl}_{3}\right) \delta 7.36(\mathrm{~s}, 1 \mathrm{H}), 7.25(\mathrm{~s}, 1 \mathrm{H}), 6.28(\mathrm{~s}, 1 \mathrm{H}), 4.39-4.33(\mathrm{~m}, 1 \mathrm{H}), 3.82-3.75(\mathrm{~m}, 1 \mathrm{H})$, $3.69-3.62(\mathrm{~m}, 1 \mathrm{H}), 2.91(\mathrm{dd}, J=14.3,3.6 \mathrm{~Hz}, 1 \mathrm{H}), 2.80(\mathrm{dd}, J=14.5,8.2 \mathrm{~Hz}, 1 \mathrm{H}), 2.24-2.09$ $(\mathrm{m}, 1 \mathrm{H}), 1.90-1.78(\mathrm{~m}, 1 \mathrm{H}), 1.45(\mathrm{~s}, 9 \mathrm{H}) .{ }^{13} \mathbf{C} \mathbf{N M R}\left(151 \mathrm{MHz}, \mathrm{CDCl}_{3}\right) \delta 156.4,143.0,140.1$, 120.0, 111.7, 79.4, 61.4, 46.3, 30.0, 28.6, 21.2. IR (neat) $v_{\max } 2973$ (w), 2927 (w), 1692 (s), 1500 (w), 1478 (w), $1455(\mathrm{w}), 1389$ (s), 1364 (s), 1306 (w), 1254 (w), 1182 (m), 1133 (s), 1069 (w), $1022(\mathrm{~m}), 972(\mathrm{w}), 947(\mathrm{w}), 872(\mathrm{~m}), 770(\mathrm{~m}), 727(\mathrm{w}), 600(\mathrm{~m}), 564(\mathrm{w}) \mathrm{cm}^{-1}$. HRMS (DART+) for $\mathrm{C}_{13} \mathrm{H}_{20} \mathrm{NO}_{3}[\mathrm{M}+\mathrm{H}]^{+}$: Calc'd: 238.1438, found: 238.1430 .

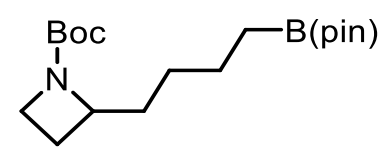

tert-butyl 2-(4-(4,4,5,5-tetramethyl-1,3,2-dioxaborolan-2yl)butyl)azetidine-1-carboxylate (5) The reaction was performed according to the general procedure above (Method $\boldsymbol{A}$ ) with tert-butyl (3,7-bis(4,4,5,5-tetramethyl-1,3,2-dioxaborolan-2yl)heptyl)(methoxy)carbamate (S-15) $(99.5 \mathrm{mg}, 0.20 \mathrm{mmol}, 1.0$ equiv.), and potassium tertbutoxide (24.7 mg, $0.22 \mathrm{mmol}, 1.1$ equiv.) in toluene and THF (3.6/0.36 mL, $0.05 \mathrm{M}$ ) for 24 hours. The compound was purified with $\mathrm{SiO}_{2}$ chromatography (10\% ethyl acetate in hexane, stain with CAM) as a colorless oil (37.3 mg, 55\%). ${ }^{1} \mathbf{H}$ NMR (400 MHz, $\left.\mathrm{CDCl}_{3}\right) \delta 4.20-4.10(\mathrm{~m}, 1 \mathrm{H}), 3.84$ - $3.72(\mathrm{~m}, 2 \mathrm{H}), 2.29-2.14(\mathrm{~m}, 1 \mathrm{H}), 1.92-1.73(\mathrm{~m}, 2 \mathrm{H}), 1.63-1.52(\mathrm{~m}, 1 \mathrm{H}), 1.46-1.37(\mathrm{~m}$, $11 \mathrm{H}), 1.32-1.25(\mathrm{~m}, 2 \mathrm{H}), 1.23(\mathrm{~s}, 12 \mathrm{H}), 0.77(\mathrm{t}, J=7.8 \mathrm{~Hz}, 2 \mathrm{H}) .{ }^{13} \mathbf{C} \mathbf{N M R}\left(151 \mathrm{MHz}, \mathrm{CDCl}_{3}\right) \delta$ 156.7, 83.0, 79.1, 62.3, 35.3, 30.5, 28.6, 27.4, 24.9, 24.2, 22.1. ${ }^{11} \mathbf{B}$ NMR $\left(160 \mathrm{MHz}, \mathrm{CDCl}_{3}\right) \delta$ 33.9. IR (neat) $v_{\max } 2976(\mathrm{w}), 2930(\mathrm{w}), 2890(\mathrm{w}), 2860(\mathrm{w}), 1698(\mathrm{~s}), 1456(\mathrm{w}), 1364(\mathrm{~s}), 1317$ (m), 1255 (w), 1142 (s), 968 (w), 881 (w), 846 (w), 777 (w) cm $\mathrm{cm}^{-1}$. HRMS (DART+) for $\mathrm{C}_{18} \mathrm{H}_{35} \mathrm{BNO}_{4}[\mathrm{M}+\mathrm{H}]^{+}$: Calc'd: 340.2654 , found: 340.2658 . 


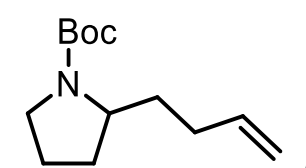

tert-butyl 2-(but-3-en-1-yl)pyrrolidine-1-carboxylate (6) The reaction was performed according to the general procedure above (Method $\boldsymbol{A})$ with tert-butyl methoxy(4(4,4,5,5-tetramethyl-1,3,2-dioxaborolan-2-yl)oct-7-en-1-yl)carbamate (S-10) (76.7 mg, 0.20 mmol, 1.0 equiv.), and potassium tert-butoxide $(24.7 \mathrm{mg}, 0.22 \mathrm{mmol}, 1.1$ equiv.) in toluene and THF (3.6/0.36 mL, $0.05 \mathrm{M})$ for 14 hours. The compound was purified with $\mathrm{SiO}_{2}$ chromatography (10\% ethyl acetate in hexane, stain with $\left.\mathrm{KMnO}_{4}\right)$ as a colorless oil $(24.0 \mathrm{mg}, 53 \%)$. ${ }^{\mathbf{1}} \mathbf{H} \mathbf{~ N M R}(500$ $\left.\mathrm{MHz} \mathrm{CDCl}_{3}\right) \delta 5.87-5.77(\mathrm{~m}, 1 \mathrm{H}), 5.06-4.98(\mathrm{~m}, 1 \mathrm{H}), 4.97-4.91(\mathrm{~m}, 1 \mathrm{H}), 3.76(\mathrm{br}, 1 \mathrm{H}), 3.36$ (br, 1H), $3.33-3.26(\mathrm{~m}, 1 \mathrm{H}), 2.11-1.96(\mathrm{~m}, 2 \mathrm{H}), 1.96-1.73(\mathrm{~m}, 4 \mathrm{H}), 1.69-1.60(\mathrm{~m}, 1 \mathrm{H}), 1.46$ $(\mathrm{s}, 9 \mathrm{H}), 1.41-1.33(\mathrm{~m}, 1 \mathrm{H}) .{ }^{13} \mathbf{C}$ NMR $\left(151 \mathrm{MHz}, \mathrm{CDCl}_{3}\right.$, rotamers) $\delta 154.8,138.5,114.6$, $79.1 \& 79.0,57.0 \& 56.9,46.6 \& 46.2,34.0 \& 33.4,30.8,29.9 \& 29.8,28.7,23.9 \& 23.2$. IR (neat) $v_{\max }$ 2972 (w), 2928 (w), 2873 (w), 1694 (s), 1641 (w), 1478 (w), 1454 (w), 1392 (m), 1365 (w), 1250 (w), $1171(\mathrm{w}), 1107(\mathrm{w}), 909(\mathrm{w}), 868(\mathrm{w}), 771(\mathrm{w}) \mathrm{cm}^{-1}$. HRMS (DART+) for $\mathrm{C}_{13} \mathrm{H}_{24} \mathrm{NO}_{2}$ $[\mathrm{M}+\mathrm{H}]^{+}$: Calc'd: 226.1802 , found: 226.1805 .

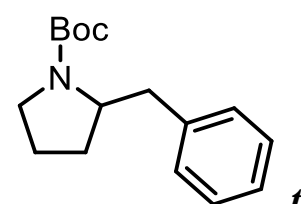

tert-butyl 2-benzylpyrrolidine-1-carboxylate (7) The reaction was performed according to the general procedure above (Method A) with tert-butyl methoxy(5-phenyl-4(4,4,5,5-tetramethyl-1,3,2-dioxaborolan-2-yl)pentyl)carbamate (S-16) (83.9 mg, $0.20 \mathrm{mmol}, 1.0$ equiv.), and potassium tert-butoxide $(24.7 \mathrm{mg}, 0.22 \mathrm{mmol}, 1.1 \mathrm{equiv}$.$) in toluene and THF$ (3.6/0.36 mL, $0.05 \mathrm{M}$ ) for 14 hours. The compound was purified with $\mathrm{SiO}_{2}$ chromatography (10\% ethyl acetate in hexane, stain with Ninhydrin) as a colorless oil (38.1 mg, 73\%). All spectral data was in accordance with previously published results. ${ }^{11}$

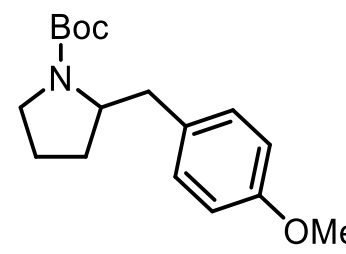

tert-butyl 2-(4-methoxybenzyl)pyrrolidine-1-carboxylate (8) The reaction was performed according to the general procedure above (Method $\boldsymbol{A}$ ) with tert-butyl methoxy(5-(4-methoxyphenyl)-4-(4,4,5,5-tetramethyl-1,3,2-dioxaborolan-2-yl)pentyl)carbamate (S-17) (89.9 mg, $0.20 \mathrm{mmol}, 1.0$ equiv.), and potassium tert-butoxide (24.7 $\mathrm{mg}, 0.22 \mathrm{mmol}, 1.1$ equiv.) in toluene and THF (3.6/0.36 mL, $0.05 \mathrm{M})$ for 14 hours. The compound was purified with $\mathrm{SiO}_{2}$ chromatography (10\% ethyl acetate in hexane, stain with Ninhydrin) as a colorless oil (38.4 $\mathrm{mg}, 66 \%$ ). All spectral data was in accordance with previously published results. ${ }^{11}$ 


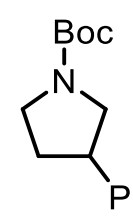

tert-butyl 3-phenylpyrrolidine-1-carboxylate (9) The reaction was performed according to the general procedure above (Method $\boldsymbol{A}$ ) with tert-butyl methoxy(2-phenyl-4(4,4,5,5-tetramethyl-1,3,2-dioxaborolan-2-yl)butyl)carbamate (S-7) (81.6 mg, $0.20 \mathrm{mmol}, 1.0$ equiv.), and potassium tert-butoxide $(24.7 \mathrm{mg}, 0.22 \mathrm{mmol}, 1.1$ equiv.) in toluene and THF (3.6/0.36 mL, $0.05 \mathrm{M}$ ) for 14 hours. The compound was purified with $\mathrm{SiO}_{2}$ chromatography (10\% ethyl acetate in hexane, stain with Ninhydrin) as a colorless oil (39.5 mg, 80\%). All spectral data was in accordance with previously published results. ${ }^{12}$<smiles>C=CCCC1CCCCN1C(=O)OC(C)(C)C</smiles>

tert-butyl 2-(but-3-en-1-yl)piperidine-1-carboxylate (10) The reaction was performed according to the general procedure above (Method $\boldsymbol{A}$ ) with tert-butyl methoxy(5(4,4,5,5-tetramethyl-1,3,2-dioxaborolan-2-yl)non-8-en-1-yl)carbamate (S-11) (79.5 mg, 0.20 mmol, 1.0 equiv.), and potassium tert-butoxide $(24.7 \mathrm{mg}, 0.22 \mathrm{mmol}, 1.1$ equiv.) in toluene and THF (3.6/0.36 mL, $0.05 \mathrm{M}$ ) for 14 hours. The compound was purified with $\mathrm{SiO}_{2}$ chromatography ( $10 \%$ ethyl acetate in hexane, stain with $\mathrm{KMnO}_{4}$ ) as a colorless oil (29.7 mg, 62\%). All spectral data was in accordance with previously published results. ${ }^{13}$<smiles>CC(C)(C)OC(=O)N1CCCCC1Cc1ccccc1</smiles>

tert-butyl 2-benzylpiperidine-1-carboxylate (11) The reaction was performed according to the general procedure above (Method $\boldsymbol{A}$ ) with tert-butyl methoxy(6-phenyl-5(4,4,5,5-tetramethyl-1,3,2-dioxaborolan-2-yl)hexyl)carbamate (S-19) (86.7 mg, 0.20 mmol, 1.0 equiv.), and potassium tert-butoxide $(24.7 \mathrm{mg}, 0.22 \mathrm{mmol}, 1.1$ equiv.) in toluene and THF (3.6/0.36 mL, $0.05 \mathrm{M}$ ) for 14 hours. The compound was purified with $\mathrm{SiO}_{2}$ chromatography (10\% ethyl acetate in hexane, stain with Ninhydrin) as a colorless oil (39.5 mg, 84\%). IR (neat) $v_{\max }$ 2974 (w), 2931 (w), 2858 (w), 1686 (s), 1454 (w), 1411 (m), 1363 (m), 1270 (m), 1160 (s), 1034 $(\mathrm{w}), 871(\mathrm{w}), 742(\mathrm{w}), 700(\mathrm{w}) \mathrm{cm}^{-1}$. All other spectral data was in accordance with previously published results. $^{14}$ 
<smiles>CC(C)(C)OC(=O)N1CCCC(c2ccccc2)C1</smiles>

tert-butyl 3-phenylpiperidine-1-carboxylate (12) The reaction was performed according to the general procedure above (Method $\boldsymbol{A}$ ) with tert-butyl methoxy(2-phenyl-5(4,4,5,5-tetramethyl-1,3,2-dioxaborolan-2-yl)pentyl)carbamate (S-8) $(83.9 \mathrm{mg}, 0.20 \mathrm{mmol}, 1.0$ equiv.), and potassium tert-butoxide $(24.7 \mathrm{mg}, 0.22 \mathrm{mmol}, 1.1$ equiv.) in toluene and THF (3.6/0.36 $\mathrm{mL}, 0.05 \mathrm{M}$ ) for 14 hours. The compound was purified with $\mathrm{SiO}_{2}$ chromatography (10\% ethyl acetate in hexane, stain with Ninhydrin) as a colorless oil (33.2 mg, 63\%). All spectral data was in accordance with previously published results. ${ }^{12}$<smiles>CC(C)(C)OC(=O)N1CCCC(Cc2ccccc2)C1</smiles>

tert-butyl 3-benzylpiperidine-1-carboxylate (13) The reaction was performed according to the general procedure above (Method $\boldsymbol{A}$ ) with tert-butyl (4-benzyl-5-(4,4,5,5tetramethyl-1,3,2-dioxaborolan-2-yl)pentyl)(methoxy)carbamate (S-18) (86.7 mg, 0.20 mmol, 1.0 equiv.), and potassium tert-butoxide $(24.7 \mathrm{mg}, 0.22 \mathrm{mmol}, 1.1$ equiv.) in toluene and THF (3.6/0.36 mL, $0.05 \mathrm{M}$ ) for 14 hours. The compound was purified with $\mathrm{SiO}_{2}$ chromatography (10\% ethyl acetate in hexane, stain with Ninhydrin) as a colorless oil (46.2 mg, 84\%). ${ }^{1} \mathbf{H}$ NMR (500 $\left.\mathrm{MHz} \mathrm{CDCl}_{3}\right) \delta 7.30$ - $7.24(\mathrm{~m}, 2 \mathrm{H}), 7.21-7.12$ (m, 3H), 4.17 - 3.66 (brm, 2H), 2.78 (brs, 1H), $2.68-2.37$ (brm, 3H), $1.79-1.69$ (m, 2H), $1.68-1.57$ (brm, 1H), 1.42 (s, 10H), 1.13 (brs, 1H). ${ }^{13}$ C NMR $\left(126 \mathrm{MHz}, \mathrm{CDCl}_{3}\right) \delta 155.0,140.1,129.2,128.4,126.1,79.3,49.7,44.6,40.3,37.8$, 30.8, 28.6, 25.0. IR (neat) $v_{\max } 2975(\mathrm{w}), 2929(\mathrm{w}), 2851$ (w), $1688(\mathrm{~s}), 1419(\mathrm{~m}), 1364(\mathrm{~m}), 1266$ (m), $1240(\mathrm{~m}), 1167(\mathrm{~m}), 1130(\mathrm{~m}), 886(\mathrm{w}), 745(\mathrm{w}), 700(\mathrm{~m}) \mathrm{cm}^{-1}$. HRMS (DART+) for $\mathrm{C}_{17} \mathrm{H}_{26} \mathrm{NO}_{2}[\mathrm{M}+\mathrm{H}]^{+}$: Calc'd: 276.1958, found: 276.1971 .

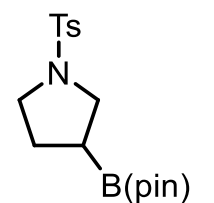

3-(4,4,5,5-tetramethyl-1,3,2-dioxaborolan-2-yl)-1-tosylpyrrolidine (15) The reaction was performed according to the general procedure above (Method B) with tert-butyl (3,4bis(4,4,5,5-tetramethyl-1,3,2-dioxaborolan-2-yl)butyl)(methoxy)carbamate (14) (91.0 mg, 0.20 mmol, 1.0 equiv.), and potassium tert-butoxide (29.2 $\mathrm{mg}, 0.26 \mathrm{mmol}, 1.3$ equiv.) in toluene and THF (3.6/0.36 mL, $0.05 \mathrm{M}$ ) for 24 hours. The compound was purified with $\mathrm{SiO}_{2}$ chromatography (15\% ethyl acetate in hexane, stain with CAM) as a white solid (35.8 mg, 51\%). ${ }^{\mathbf{1}} \mathbf{H}$ NMR (500 $\left.\mathrm{MHz} \mathrm{CDCl}_{3}\right) \delta 7.71(\mathrm{~d}, J=7.9 \mathrm{~Hz}, 2 \mathrm{H}), 7.30(\mathrm{~d}, J=7.9 \mathrm{~Hz}, 2 \mathrm{H}), 3.47$ (t, $\left.J=9.2 \mathrm{~Hz}, 1 \mathrm{H}\right), 3.37-$ $3.27(\mathrm{~m}, 1 \mathrm{H}), 3.19-3.09(\mathrm{~m}, 2 \mathrm{H}), 2.42(\mathrm{~s}, 3 \mathrm{H}), 1.97-1.87(\mathrm{~m}, 1 \mathrm{H}), 1.75-1.63(\mathrm{~m}, 1 \mathrm{H}), 1.48-$ $1.38(\mathrm{~m}, 1 \mathrm{H}), 1.18$ (s, 12H). ${ }^{13} \mathbf{C}$ NMR $\left(126 \mathrm{MHz} \mathrm{CDCl}_{3}\right) \delta 143.2,134.5,129.7,127.7,83.8,50.1$, 
48.7, 28.0, 24.8, 24.8, 21.6. ${ }^{11} \mathbf{B}$ NMR $\left(160 \mathrm{MHz}, \mathrm{CDCl}_{3}\right) \delta$ 33.9. IR (neat) $v_{\max } 2976(\mathrm{w}), 2929$ (w), 2883 (w), 1597 (w), 1452 (w), 1382 (m), 1326 (s), 1230 (w), 1158 (s), 1142 (s), 1098 (m), 1026 (w), 1015 (w), 972 (w), 855 (w), 816 (w), 775 (w), 709 (w), 661 (s), 591 (s), 548 (s) cm c $^{-1}$ HRMS (DART+) for $\mathrm{C}_{17} \mathrm{H}_{27} \mathrm{BNO}_{4} \mathrm{~S}[\mathrm{M}+\mathrm{H}]^{+}$: Calc'd: 352.1748 , found: 352.1750 . Melting point: $73-74{ }^{\circ} \mathrm{C}$.<smiles>O=C(O)N1CCCC1CBr</smiles>

tert-butyl 2-((4,4,5,5-tetramethyl-1,3,2-dioxaborolan-2-yl)methyl)pyrrolidine-1-carboxylate (17)

The reaction was performed according to the general procedure above (Method $\boldsymbol{A}$ ) with tert-butyl (4,5-bis(4,4,5,5-tetramethyl-1,3,2-dioxaborolan-2-yl)pentyl)(methoxy)carbamate (16) (93.9 mg, $0.20 \mathrm{mmol}, 1.0$ equiv.), and potassium tert-butoxide (38.2 $\mathrm{mg}, 0.34 \mathrm{mmol}, 1.7$ equiv.) in toluene and THF $(0.9 / 0.09 \mathrm{~mL}, 0.2 \mathrm{M})$ for 24 hours. The compound was purified with $\mathrm{SiO}_{2}$ chromatography (10\% ethyl acetate in hexane, stain with CAM) as a white solid (28.6 mg, 46\%). Melting point: $63-66^{\circ} \mathrm{C}$. All spectral data was in accordance with previously published results. ${ }^{15}$<smiles>O=C(O)c1ccccc1CC1CCCCN1C(=O)c1ccccc1</smiles>

\section{tert-butyl 2-((4,4,5,5-tetramethyl-1,3,2-dioxaborolan-2-yl)methyl)piperidine-1-carboxylate (19)}

The reaction was performed according to the general procedure above (Method $\boldsymbol{A}$ ) with tert-butyl (5,6-bis(4,4,5,5-tetramethyl-1,3,2-dioxaborolan-2-yl)hexyl)(methoxy)carbamate (18) (96.7 mg, $0.20 \mathrm{mmol}, 1.0$ equiv.), and potassium tert-butoxide (33.7 $\mathrm{mg}, 0.30 \mathrm{mmol}, 1.5$ equiv.) in toluene and THF $(0.9 / 0.09 \mathrm{~mL}, 0.2 \mathrm{M})$ for 24 hours. The compound was purified with $\mathrm{SiO}_{2}$ chromatography (10\% ethyl acetate in hexane, stain with CAM) as a colorless oil (32.0 mg, 49\%). ${ }^{1} \mathbf{H}$ NMR $\left(600 \mathrm{MHz}, \mathrm{CDCl}_{3}\right) \delta 4.49$ (brs, 1H), 3.88 (brd, $\left.J=12.2 \mathrm{~Hz}, 1 \mathrm{H}\right), 2.81$ (t, $J=13.3,13.3$ $\mathrm{Hz}, 1 \mathrm{H}), 1.63-1.48(\mathrm{~m}, 5 \mathrm{H}), 1.44(\mathrm{~s}, 9 \mathrm{H}), 1.39-1.30(\mathrm{~m}, 1 \mathrm{H}), 1.29-1.22(\mathrm{~m}, 1 \mathrm{H}), 1.22(\mathrm{~s}, 6 \mathrm{H})$, $1.22(\mathrm{~s}, 6 \mathrm{H}), 1.00(\mathrm{dd}, J=15.3,5.8 \mathrm{~Hz}, 1 \mathrm{H}) .{ }^{13} \mathbf{C ~ N M R}\left(126 \mathrm{MHz}, \mathrm{CDCl}_{3}\right) \delta 154.9,83.2,79.0$, 47.8, 38.8, 30.1, 28.7, 25.9, 25.0, 24.9, 18.8. ${ }^{11} \mathbf{B} \mathbf{N M R}\left(160 \mathrm{MHz}, \mathrm{CDCl}_{3}\right) \delta$ 34.0. IR (neat) $v_{\max }$ 2975 (w), 2930 (w), 2856 (w), 1687 (s), 1470 (w), 1447 (w), 1363 (s), 1322 (s), 1269 (m), 1253 (m), 1150 (s), 1141 (s), 1097 (w), 1068 (w), 1039 (w), 1003 (w), 968 (m), 927 (w), 872 (m), 847 (m), $769(\mathrm{w}) \mathrm{cm}^{-1}$. HRMS (DART+) for $\mathrm{C}_{17} \mathrm{H}_{33} \mathrm{BNO}_{4}[\mathrm{M}+\mathrm{H}]^{+}$: Calc'd: 326.2497, found: 326.2501 . 


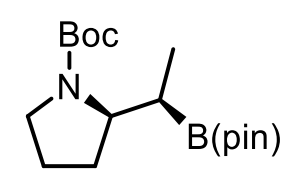

\section{tert-butyl rac-(R)-2-((R)-1-(4,4,5,5-tetramethyl-1,3,2-dioxaborolan-2-yl)ethyl)pyrrolidine-1- carboxylate (31)}

The reaction was performed according to the general procedure above (Method $A$ ) with tert-butyl (rac-(4S,5S)-4,5-bis(4,4,5,5-tetramethyl-1,3,2-dioxaborolan-2-yl)hexyl)(methoxy)carbamate (30) (96.7 mg, $0.20 \mathrm{mmol}, 1.0$ equiv.), and potassium tert-butoxide (33.7 $\mathrm{mg}, 0.30 \mathrm{mmol}, 1.5$ equiv.) in toluene and THF $(0.9 / 0.09 \mathrm{~mL}, 0.2 \mathrm{M})$ for 24 hours. The compound was purified with $\mathrm{SiO}_{2}$ chromatography (10\% ethyl acetate in hexane, stain with CAM) as a colorless oil (34.2 mg, 53\%). ${ }^{1}$ H NMR (500 MHz, $\mathrm{CDCl}_{3}$, rotamers) $\delta 3.98$ (brs, 1H), 3.57 (brs, $0.5 \mathrm{H}$ ), 3.42 (brs, $\left.0.5 \mathrm{H}\right), 3.26$ - $3.12(\mathrm{~m}, 1 \mathrm{H}), 2.06-1.92(\mathrm{~m}, 1 \mathrm{H}), 1.91-1.73(\mathrm{~m}, 2 \mathrm{H}), 1.73-1.53(\mathrm{~m}, 2 \mathrm{H}), 1.44(\mathrm{~s}, 9 \mathrm{H}), 1.21$ $(\mathrm{s}, 12 \mathrm{H}), 0.84(\mathrm{~d}, J=7.4 \mathrm{~Hz}, 3 \mathrm{H}) .{ }^{13} \mathrm{C} \mathrm{NMR}\left(151 \mathrm{MHz}, \mathrm{CDCl}_{3}\right.$, rotamers) $\delta 155.0 \& 154.7$, 83.1\&82.6, 79.0, 59.3\&58.4, 47.6, 29.4, 28.7, 24.9, 24.4\&24.2, 21.1, 9.7, 8.5. ${ }^{11} \mathrm{~B}$ NMR (160 MHz, $\left.\mathrm{cdcl}_{3}\right) \delta 33.9$. IR (neat) $v_{\max } 2975(\mathrm{w}), 2931(\mathrm{w}), 2876(\mathrm{w}), 1692(\mathrm{~s}), 1458(\mathrm{w}), 1392(\mathrm{~s}), 1366(\mathrm{~s})$, 1317 (w), 1269 (w), 1168 (m), 1145 (m), 1107 (m), 868 (w) cm $\mathrm{cm}^{-1}$. HRMS (DART+) for $\mathrm{C}_{17} \mathrm{H}_{33} \mathrm{BNO}_{4}[\mathrm{M}+\mathrm{H}]^{+}$: Calc'd: 326.2497 , found: 326.2505 .

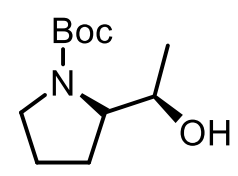

tert-butyl rac-(R)-2-((R)-1-hydroxyethyl)pyrrolidine-1-carboxylate (32) Tertbutyl $\quad r a c-(R)-2-((R)-1-(4,4,5,5$-tetramethyl-1,3,2-dioxaborolan-2-yl)ethyl)pyrrolidine-1carboxylate (31) $(34.2 \mathrm{mg}, 0.105 \mathrm{mmol}, 1.0$ equiv.) was dissolved in THF ( $1 \mathrm{~mL})$. At $0{ }^{\circ} \mathrm{C}$, the solution was treated carefully with $3 \mathrm{M} \mathrm{NaOH}(0.4 \mathrm{~mL}), \mathrm{pH} 7.00$ buffer solution $(0.4 \mathrm{~mL})$ and $30 \%$ $\mathrm{H}_{2} \mathrm{O}_{2}(0.2 \mathrm{~mL})$. The cooling bath was removed, and the reaction was allowed to stir at r.t. for 2 hours. At this time the reaction was again cooled down to $0{ }^{\circ} \mathrm{C}$ and carefully quenched with saturated aqueous sodium thiosulfate $(0.5 \mathrm{~mL})$. The aqueous phase was extracted with ethyl acetate ( 3 x $4 \mathrm{~mL}$ ). The combined organic layers were dried over anhydrous sodium sulfate, filtered, concentrated under reduced pressure. The compound was purified with $\mathrm{SiO}_{2}$ chromatography (40\% ethyl acetate in hexane, stain with ninhydrin) as a colorless oil (18.6 mg, 82\%). ${ }^{1} \mathbf{H}$ NMR $\left(600 \mathrm{MHz}, \mathrm{CDCl}_{3}\right) \delta 3.76-3.69(\mathrm{~m}, 1 \mathrm{H}), 3.69-3.62(\mathrm{~m}, 1 \mathrm{H}), 3.54-3.46(\mathrm{~m}, 1 \mathrm{H}), 3.31-3.23$ $(\mathrm{m}, 1 \mathrm{H}), 2.01-1.91(\mathrm{~m}, 1 \mathrm{H}), 1.86-1.78(\mathrm{~m}, 1 \mathrm{H}), 1.78-1.71(\mathrm{~m}, 1 \mathrm{H}), 1.62(\mathrm{~s}, 1 \mathrm{H}), 1.47(\mathrm{~s}, 9 \mathrm{H})$, $1.16-1.13$ (m, 3H). IR (neat) $v_{\max } 3420$ (br), 2974 (w), 2929 (w), 1683 (s), 1668 (s), 1456 (w), 1401 (s), 1366 (w), 1168 (m), 1106 (w). HRMS (DART+) for $\mathrm{C}_{11} \mathrm{H}_{22} \mathrm{NO}_{3}[\mathrm{M}+\mathrm{H}]^{+}$: Calc'd: 216.1594, found: 216.1600 . 


\section{Synthesis of Enantiomerically Enriched Compounds and Testing of Stereospecificity}<smiles>CON(CCBr)CCC(Br)Br</smiles>

tert-butyl

(S)-(3,4-bis $(4,4,5,5$-tetramethyl-1,3,2-dioxaborolan-2-

yl)butyl)(methoxy)carbamate (21) The reaction was performed according to a procedure reported in the literature ${ }^{1}$ with slight modification. In the glovebox, an oven-dried $20 \mathrm{~mL}$ vial was loaded with bis(pinacolato)diboron ( $0.28 \mathrm{~g}, 1.1 \mathrm{mmol}, 1.1$ equiv.), $\mathrm{Pt}(\mathrm{dba})_{3}(9.0 \mathrm{mg}, 0.01 \mathrm{mmol}$, $1.0 \mathrm{~mol} \%),(S, S)-3,5$-diethylphenylTADDOLPPh $(9.6 \mathrm{mg}, 0.012 \mathrm{mmol}, 1.2 \mathrm{~mol} \%)$ and anhydrous THF ( $5 \mathrm{~mL}$ ). The vial was sealed with a pierceable PTFE cap, taken out of the glovebox and heated at $80{ }^{\circ} \mathrm{C}$ for 20 mins. After the reaction was removed from the oil bath and cooled down to r.t., tert-butyl but-3-en-1-yl(methoxy)carbamate (20) $(0.20 \mathrm{~g}, 1.0 \mathrm{mmol}, 1.0$ equiv) was added via syringe and the reaction was heated at $60{ }^{\circ} \mathrm{C}$ for 14 hours. The vial was cooled down to r.t. and opened to air. The solvent was removed under reduced pressure. The compound was purified with $\mathrm{SiO}_{2}$ chromatography (10\% ethyl acetate in hexane, stain with CAM) as a yellow oil $(0.42 \mathrm{~g}, 92 \%)$. $[\boldsymbol{\alpha}] \mathbf{D}^{\mathbf{2 0}}:+2.2\left(c=0.89, \mathrm{CHCl}_{3}, l=50 \mathrm{~mm}\right)$. All other spectral data are in accordance with the racemic compound reported above (14). 


\section{Analysis of Stereochemistry:}

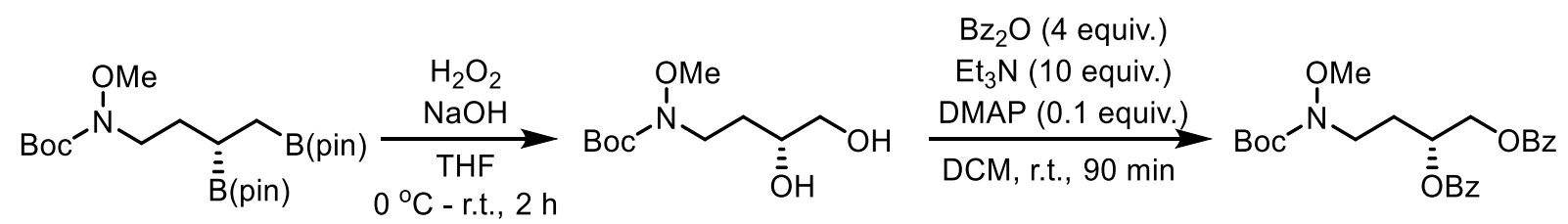

The compound was oxidized with hydrogen peroxide and sodium hydroxide, and the resulting diol was treated with benzoic anhydride, triethylamine and 4-dimethylaminopyridine. The resulting protected diol was compared with the racemic compound prepared using the same method from 14.

Chiral HPLC (Chiracel IF, 10\% isopropanol in hexanes, $0.8 \mathrm{~mL} / \mathrm{min}, 35^{\circ} \mathrm{C}, 210-290 \mathrm{~nm}$ ), analysis of tert-butyl (S)-(3,4-bis(4,4,5,5-tetramethyl-1,3,2-dioxaborolan-2-yl)butyl)(methoxy)carbamate

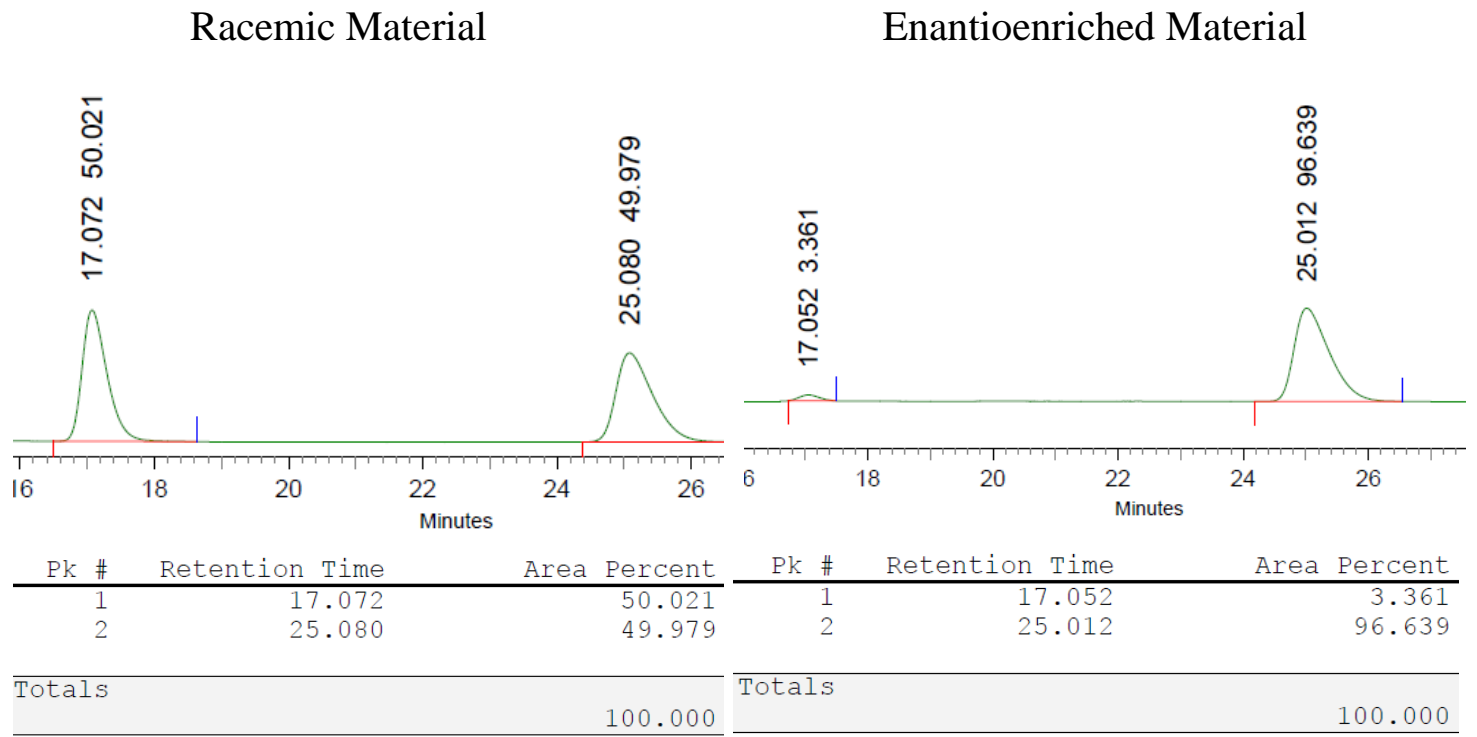


<smiles>CON(CCC[C@@H](Br)CBr)C(=O)OC(C)(C)C</smiles>

tert-butyl (S)-(4,5-bis (4,4,5,5-tetramethyl-1,3,2-dioxaborolan-2yl)pentyl)(methoxy)carbamate (26) The reaction was performed using the same method as (21) with tert-butyl methoxy(pent-4-en-1-yl)carbamate (25) $(0.22 \mathrm{~g}, 1.0 \mathrm{mmol}, 1.0$ equiv), bis(pinacolato)diboron (0.28 g, $1.1 \mathrm{mmol}, 1.1$ equiv.), $\mathrm{Pt}(\mathrm{dba})_{3}(9.0 \mathrm{mg}, 0.01 \mathrm{mmol}, 1.0 \mathrm{~mol} \%)$, $(S, S)$-3,5-diethylphenylTADDOLPPh $(9.6 \mathrm{mg}, 0.012 \mathrm{mmol}, 1.2 \mathrm{~mol} \%)$ and anhydrous THF (5 $\mathrm{mL})$. The compound was purified with $\mathrm{SiO}_{2}$ chromatography $(10 \%$ ethyl acetate in hexane, stain

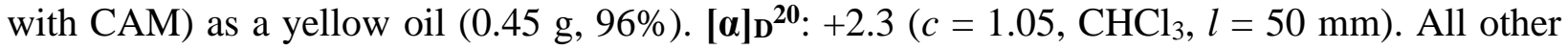
spectral data are in accordance with the racemic compound reported above (16).

\section{Analysis of Stereochemistry:}

The compound was converted to the protected diol using the same method as (21). The resulting protected diol was compared with the racemic compound prepared using the same method from 16.

Chiral HPLC (Chiracel IF, 10\% isopropanol in hexanes, $0.8 \mathrm{~mL} / \mathrm{min}, 35^{\circ} \mathrm{C}, 210-290 \mathrm{~nm}$ ), analysis of tert-butyl (S)-(4,5-bis(4,4,5,5-tetramethyl-1,3,2-dioxaborolan-2-yl)pentyl)(methoxy)carbamate
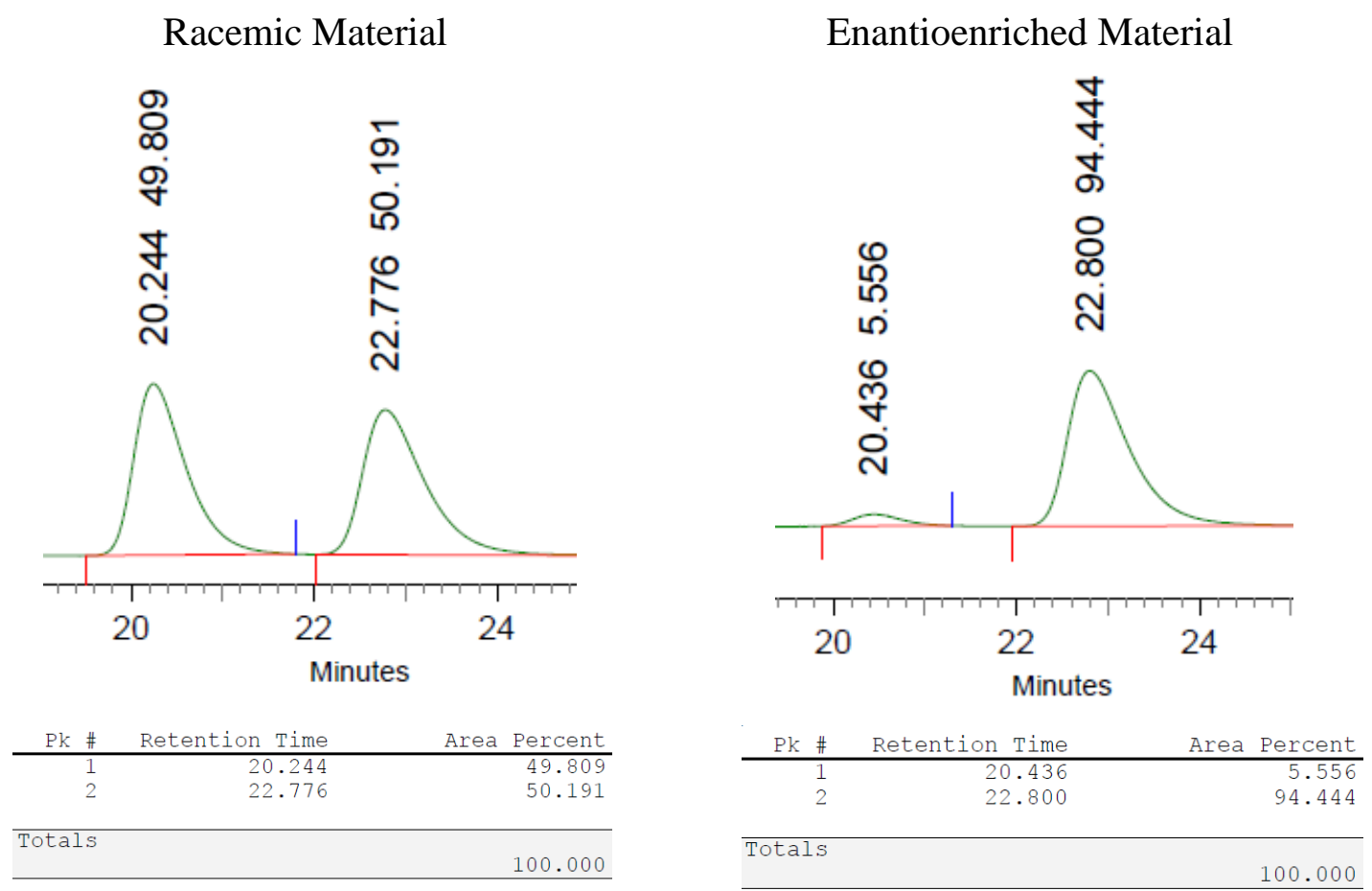
<smiles>CON(CCC(Br)Cc1ccccc1)C(C)(C)C</smiles>

tert-butyl (S)-methoxy(4-phenyl-3-(4,4,5,5-tetramethyl-1,3,2dioxaborolan-2-yl)butyl)carbamate (23) The reaction was performed according to General procedure $\quad E$ with tert-butyl $(S)$-(3,4-bis(4,4,5,5-tetramethyl-1,3,2-dioxaborolan-2yl)butyl)(methoxy)carbamate (21) (0.32 g, $0.7 \mathrm{mmol}, 1.0$ equiv.), solid potassium hydroxide (0.12 g, $2.1 \mathrm{mmol}, 3$ equiv.), $\mathrm{Pd}(\mathrm{OAc})_{2}$ (3.9 mg, $\left.0.018 \mathrm{mmol}, 2.5 \mathrm{~mol} \%\right)$, RuPhos (8.2 mg, $0.018 \mathrm{mmol}$, $2.5 \mathrm{~mol} \%)$ and bromobenzene (0.16 g, $1.5 \mathrm{mmol}, 1.5$ equiv.) in THF (6 mL) and $\mathrm{H}_{2} \mathrm{O}(0.6 \mathrm{~mL})$. The compound was purified with $\mathrm{SiO}_{2}$ chromatography $(10 \%$ ethyl acetate in hexane, stain with $\mathrm{CAM})$ as a colorless oil $(0.24 \mathrm{~g}, 84 \%)$. $[\boldsymbol{\alpha}]_{\mathbf{D}^{\mathbf{2 0}}:}+5.4\left(c=1.00, \mathrm{CHCl}_{3}, l=50 \mathrm{~mm}\right)$. All other spectral data are in accordance with the racemic compound reported above $(\mathbf{S}-12)$. 


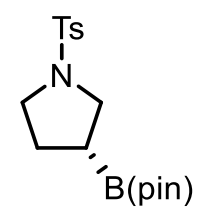

(R)-3-(4,4,5,5-tetramethyl-1,3,2-dioxaborolan-2-yl)-1-tosylpyrrolidine (22) The reaction was performed according to the general procedure above (Method B) with tert-butyl $(S)$ (3,4-bis(4,4,5,5-tetramethyl-1,3,2-dioxaborolan-2-yl)butyl)(methoxy)carbamate (21) (91.0 mg, $0.20 \mathrm{mmol}, 1.0$ equiv.), and potassium tert-butoxide (29.2 $\mathrm{mg}, 0.26 \mathrm{mmol}, 1.3$ equiv.) in toluene and THF $(3.6 / 0.36 \mathrm{~mL}, 0.05 \mathrm{M})$ for 24 hours. The compound was purified with $\mathrm{SiO}_{2}$ chromatography (15\% ethyl acetate in hexane, stain with CAM) as a colorless oil (37.0 mg, 54\%). $[\boldsymbol{\alpha}] \mathbf{D}^{\mathbf{2 0}}:-9.3\left(c=0.50, \mathrm{CHCl}_{3}, l=50 \mathrm{~mm}\right)$. All other spectral data are in accordance with the racemic compound reported above (15).

\section{Analysis of Stereochemistry:}

Enantiomeric ratio was determined in comparison to the racemic compound (15).

Chiral SFC (Chiracel OJ-H, 3\% isopropanol, $3.0 \mathrm{~mL} / \mathrm{min}, 35^{\circ} \mathrm{C}, 210-290 \mathrm{~nm}$ ), analysis of (R)-3(4,4,5,5-tetramethyl-1,3,2-dioxaborolan-2-yl)-1-tosylpyrrolidine

Racemic Material

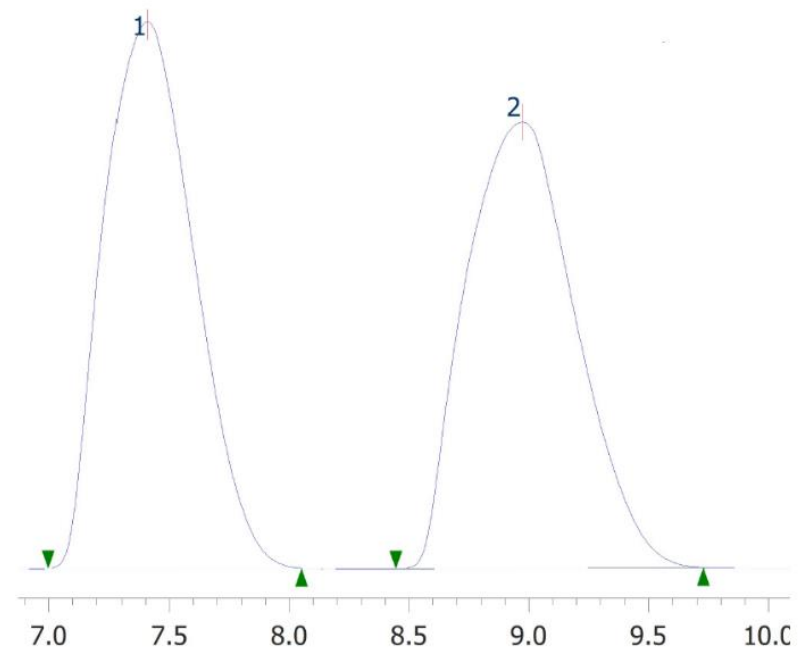

Enantioenriched Material

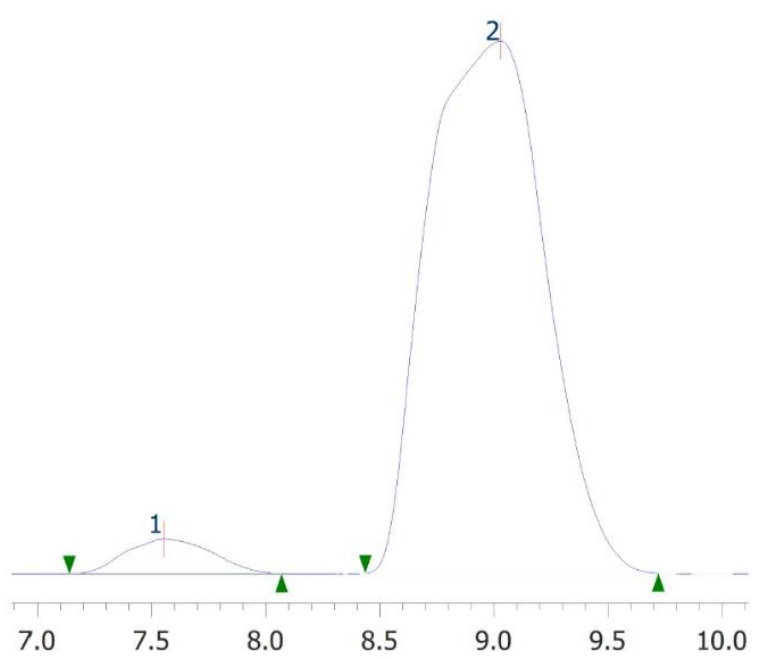

\begin{tabular}{|l|l|l|}
\hline tR [min] & Area $[\mu \mathrm{V} \cdot \mathrm{sec}]$ & Area\% \\
\hline
\end{tabular}

\begin{tabular}{|l|r|r|r|}
\hline 1 & 7.407 & 9294435 & 50.025 \\
\hline
\end{tabular}

\begin{tabular}{|l|l|l|}
\hline$\#$ tR [min] & Area $[\mu \mathrm{V} \cdot \mathrm{sec}]$ & Area\% \\
\hline
\end{tabular}

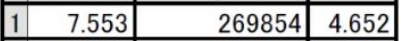

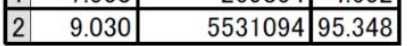




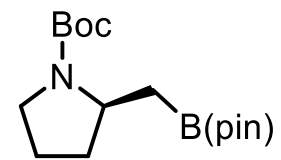

tert-butyl

(S)-2-((4,4,5,5-tetramethyl-1,3,2-dioxaborolan-2-yl)methyl)pyrrolidine-1carboxylate (27)

The reaction was performed according to the general procedure above (Method $A$ ) with tert-butyl (S)-(4,5-bis(4,4,5,5-tetramethyl-1,3,2-dioxaborolan-2-yl)pentyl)(methoxy)carbamate (26) (93.9 $\mathrm{mg}, 0.20 \mathrm{mmol}, 1.0$ equiv.), and potassium tert-butoxide (38.2 $\mathrm{mg}, 0.34 \mathrm{mmol}, 1.7$ equiv.) in toluene and THF $(0.9 / 0.09 \mathrm{~mL}, 0.2 \mathrm{M})$ for 24 hours. The compound was purified with $\mathrm{SiO}_{2}$ chromatography (10\% ethyl acetate in hexane, stain with CAM) as a colorless oil (27.2 mg, 44\%). $[\boldsymbol{\alpha}]_{\mathbf{D}} \mathbf{2 0}^{20}+18.4\left(c=0.79, \mathrm{CHCl}_{3}, l=50 \mathrm{~mm}\right)$. All other spectral data are in accordance with the racemic compound reported above $(\mathbf{1 7})$.

\section{Analysis of Stereochemistry:}

Enantiomeric ratio was determined using the tosyl-protected pyrrolidine synthesized following Method B. Racemic compound was prepared using the same method from $\mathbf{1 6 .}$

Chiral SFC (Chiracel OJ-H, 3\% isopropanol, $3.0 \mathrm{~mL} / \mathrm{min}, 35^{\circ} \mathrm{C}, 210-290 \mathrm{~nm}$ ), analysis of (S)-2((4,4,5,5-tetramethyl-1,3,2-dioxaborolan-2-yl)methyl)pyrrolidine-1-carboxylate

Racemic Material Enantioenriched Material

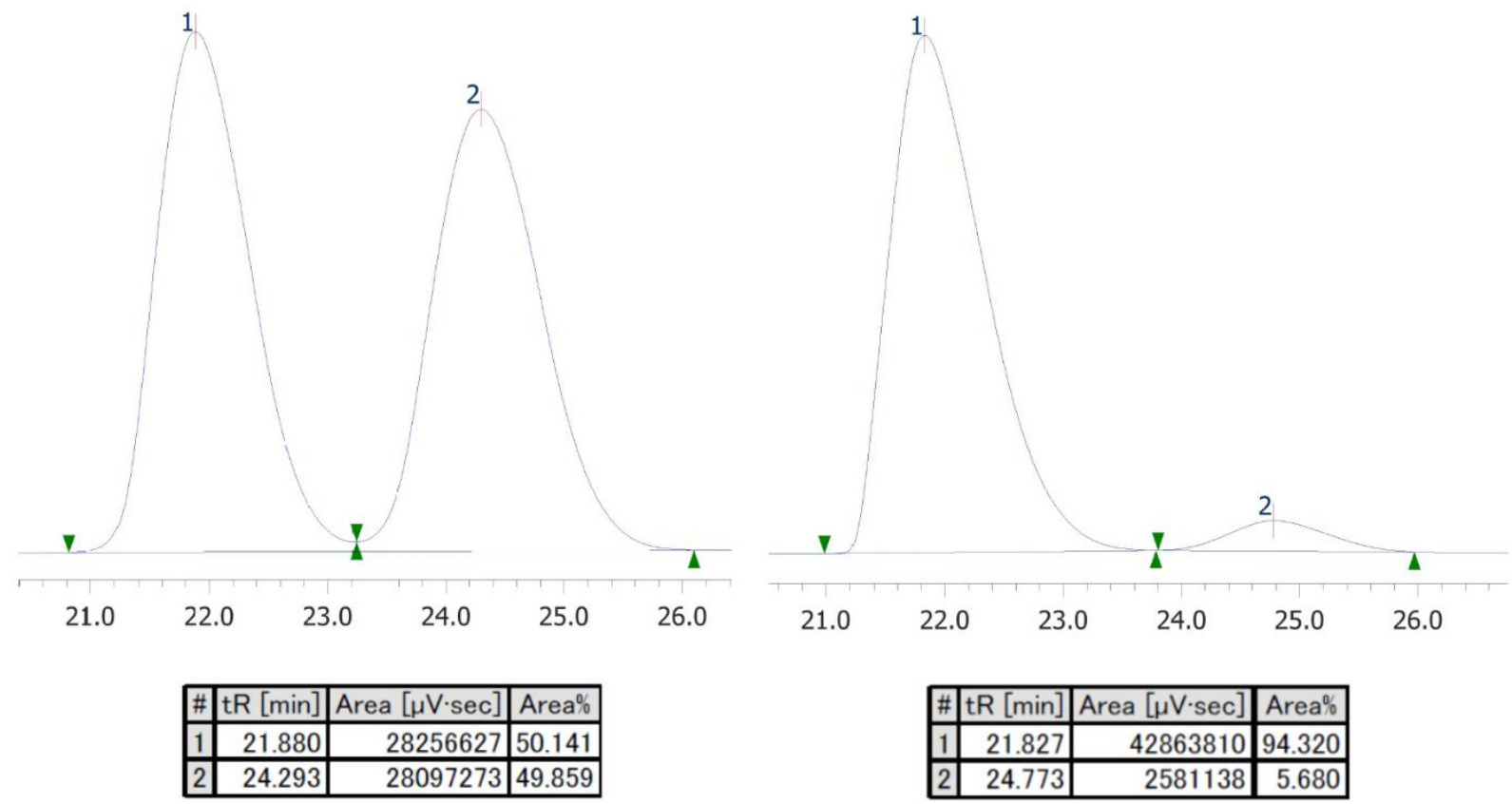


tert-butyl (S)-2-benzylazetidine-1-carboxylate (24) The reaction was performed according to the general procedure above (Method $\boldsymbol{A}$ ) with tert-butyl $(S)$-methoxy(4-phenyl-3(4,4,5,5-tetramethyl-1,3,2-dioxaborolan-2-yl)butyl)carbamate (23) (83.9 mg, $0.20 \mathrm{mmol}, 1.0$ equiv.), and potassium tert-butoxide (24.7 mg, $0.22 \mathrm{mmol}, 1.1$ equiv.) in toluene and THF (3.6/0.36 mL, $0.05 \mathrm{M}$ ) for 24 hours. The compound was purified with $\mathrm{SiO}_{2}$ chromatography (10\% ethyl acetate in hexane, stain with Ninhydrin) as a colorless oil $(32.4 \mathrm{mg}, 66 \%)$. $[\boldsymbol{\alpha}]]^{\mathbf{2 0}}:+90.0(c=$ $\left.0.50, \mathrm{CHCl}_{3}, l=50 \mathrm{~mm}\right)$.

\section{Analysis of Stereochemistry:}

Enantiomeric ratio was determined in comparison to the racemic compound ().

Chiral SFC (Chiracel OJ-H, 5\% isopropanol, $1.0 \mathrm{~mL} / \mathrm{min}, 35^{\circ} \mathrm{C}, 210-290 \mathrm{~nm}$ ), analysis of tertbutyl (S)-2-benzylazetidine-1-carboxylate

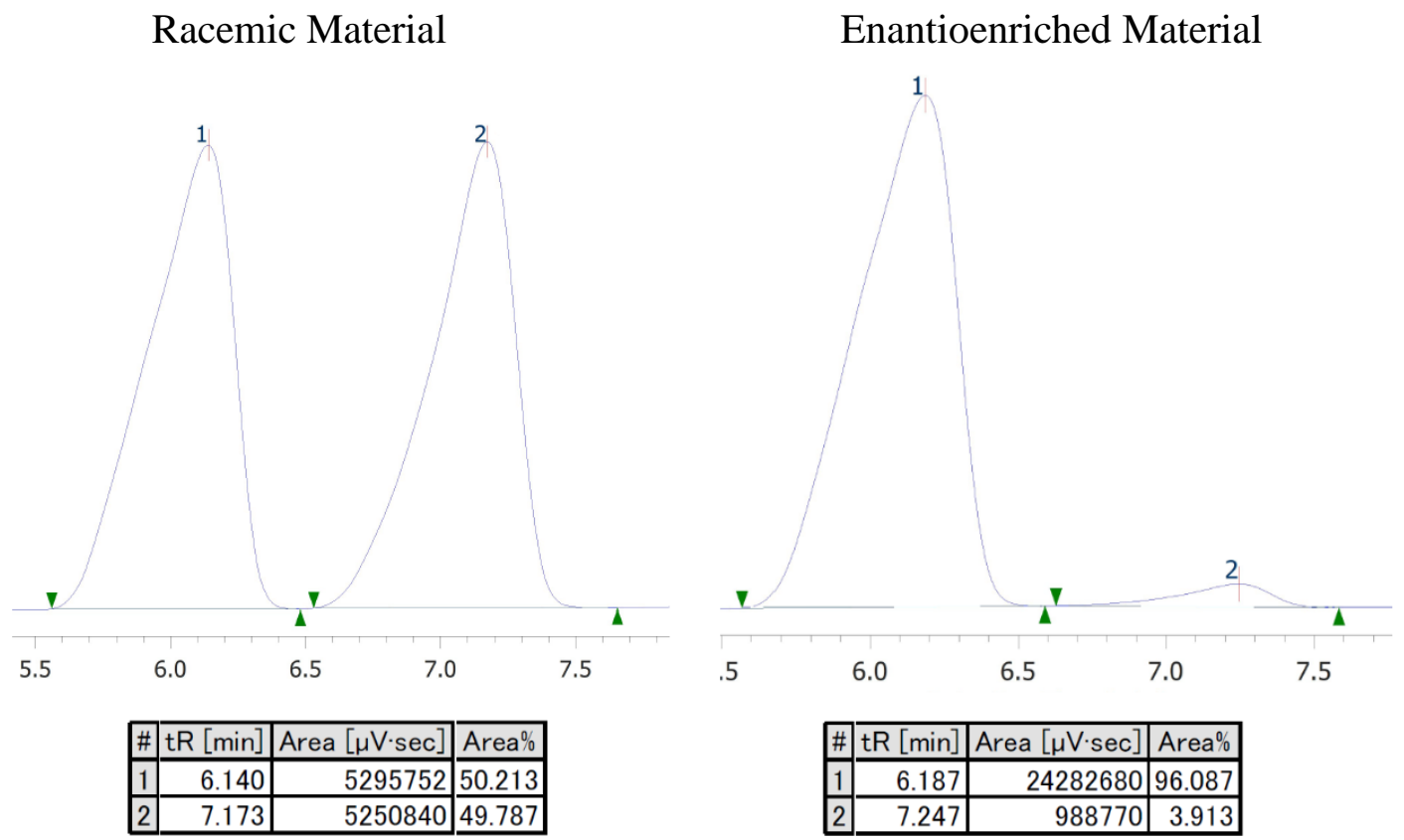


Xu, Zhang, Ingoglia, Allais, Dechert-Schmitt, Singer and Morken Supporting Information

\section{References:}

1. Coombs, J. R.; Haeffner, F.; Kliman, L. T.; Morken, J. P. J. Am. Chem. Soc. 2013, 135, 1122211231.

2. Kliman, L. T.; Mlynarski, S. N.; Morken, J. P. J. Am. Chem. Soc. 2009, 131, 13210-13211.

3. Array Biopharma, Inc.; Ahrendt, K.; Delisle, R.; Hans, J.; Lyssikatos, J. P.; Robinson, J. E.; Wallace, E. M.; Zhao, Q. WO2008/42928, 2008.

4. Joe, C. L.; Blaisdell, T. P.; Geoghan, A. F.; Tan, K. L. J. Am. Chem. Soc. 2014, 136, 8556-8559.

5. Yakura, T.; Horiuchi, Y.; Nishimura, Y.; Yamada, A.; Nambu, H.; Fujiwara, T. Adv. Synth. Catal. 2016, 358, $869-873$.

6. Chen, Y.; Bilban, M.; Foster, C. A.; Boger, D. L. J. Am. Chem. Soc. 2002, 124, 5431-5440.

7. Bonet A.; Pubill-Ulldemolins, C.; Bo, C.; Gulyás, H.; Fernández, E. Angew. Chem. Int. Ed. 2011, 50, 7158-7161.

8. Yamamoto, Y.; Fujiwara, R.; Umemoto, T.; Miyuara, N. Tetrahedron, 2004, 60, 10695-10700.

9. Mlynarshi, S. N.; Schuster, C. H.; Morken, J. P. Nature, 2014, 505, 386-390.

10. Qiu, Z.; Zhu, M.; Zheng, L.; Li, J.; Zou, D.; Wu, Y.; Wu, Y. Tetrahedron. Lett., 2019, 60, 1321-1324.

11. Angelini, L.; Davies, J.; Simonetti, M.; Sanz, L. M.; Sheikh, N. S.; Leonori, D. Angew. Chem. Int. Ed. 2019, 58, 5003-5007.

12. Barré, B.; Gonnard, L.; Campagne, R.; Reymond, S.; Marin, J.; Ciapetti, P.; Brellier, M.; Guérinot, A.; Cossy, J. Org. Lett. 2014, 16, 6160-6163.

13. Cheng, J.; Zheng, X.; Huang, P. Tetrahedron, 2019, 75, 1612-1623.

14. Sun, Z.; Tang, B.; Liu, K.; Zhu, H. Y. Chem. Commun. 2020, 56, 1294-1297.

15. Georgiou, I.; Whiting, A. Eur. J. Org. Chem. 2012, 22, 4110-4113. 


\section{Spectral data}

${ }^{1} \mathbf{H}$ NMR (600 MHz, $\left.\mathrm{CDCl}_{3}\right)(\mathbf{2 0})$

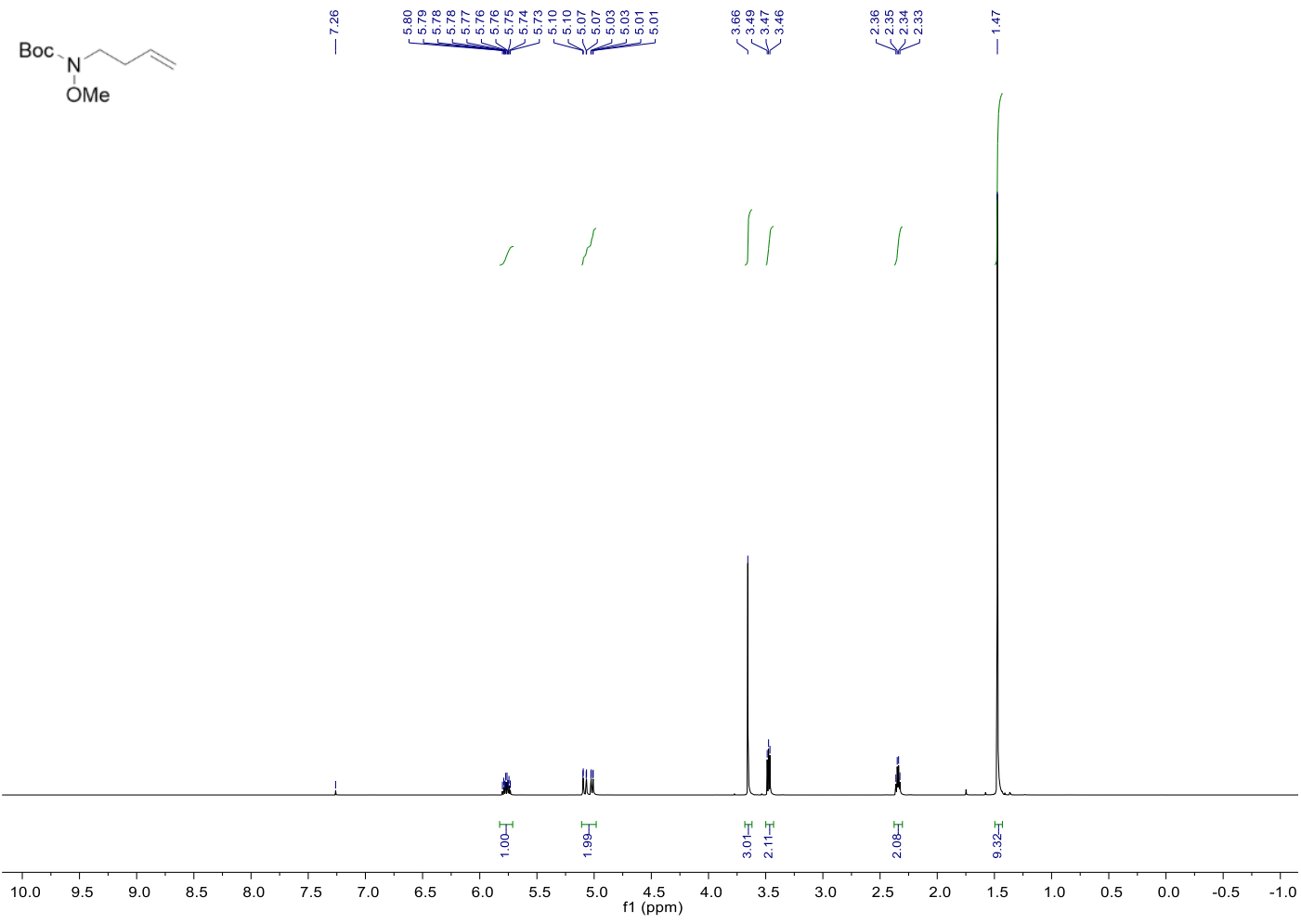

${ }^{13} \mathrm{C}$ NMR (151 MHz, $\left.\mathrm{CDCl}_{3}\right)(\mathbf{2 0})$

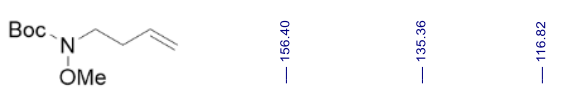

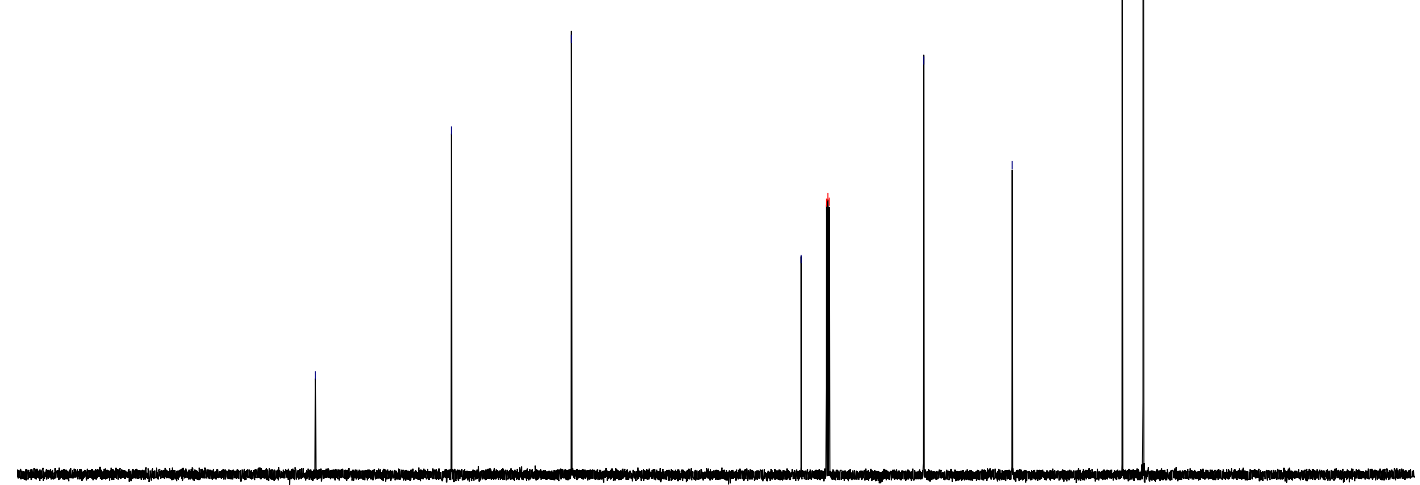

200
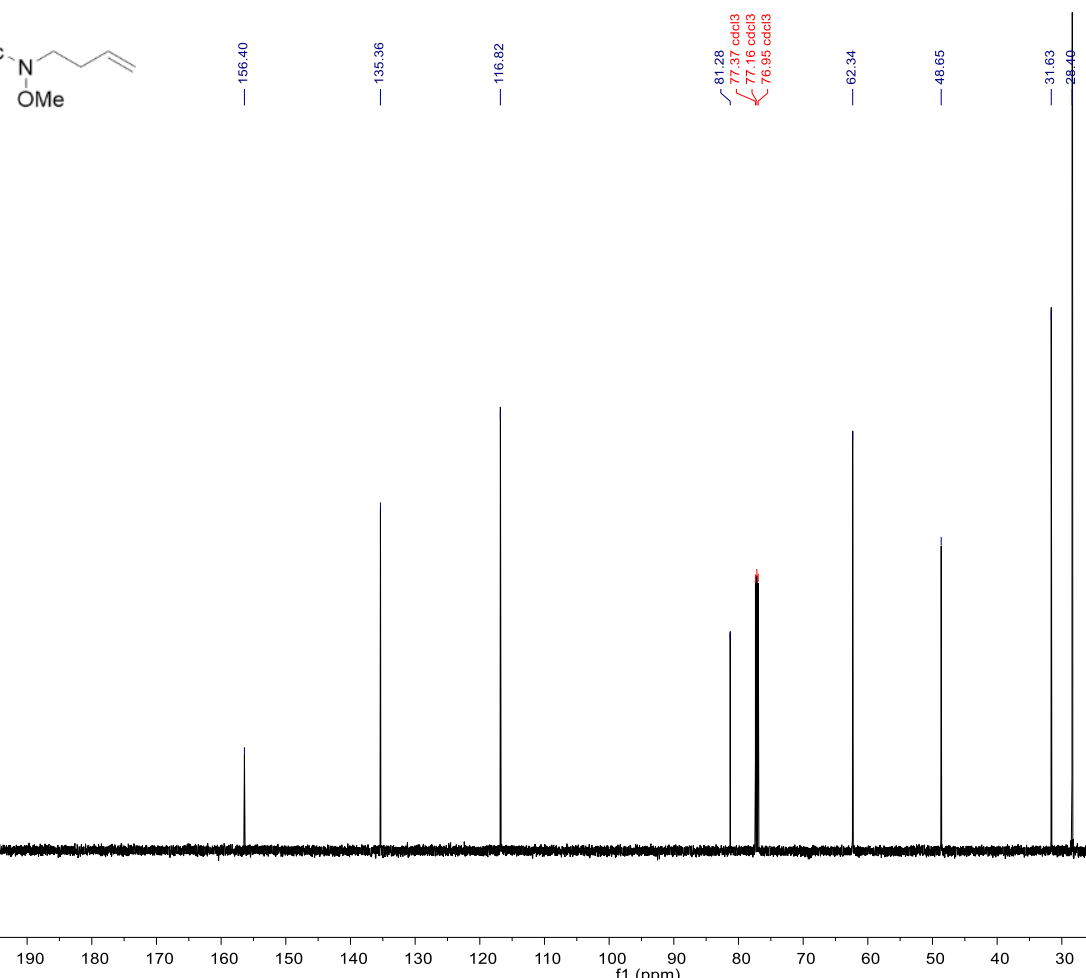


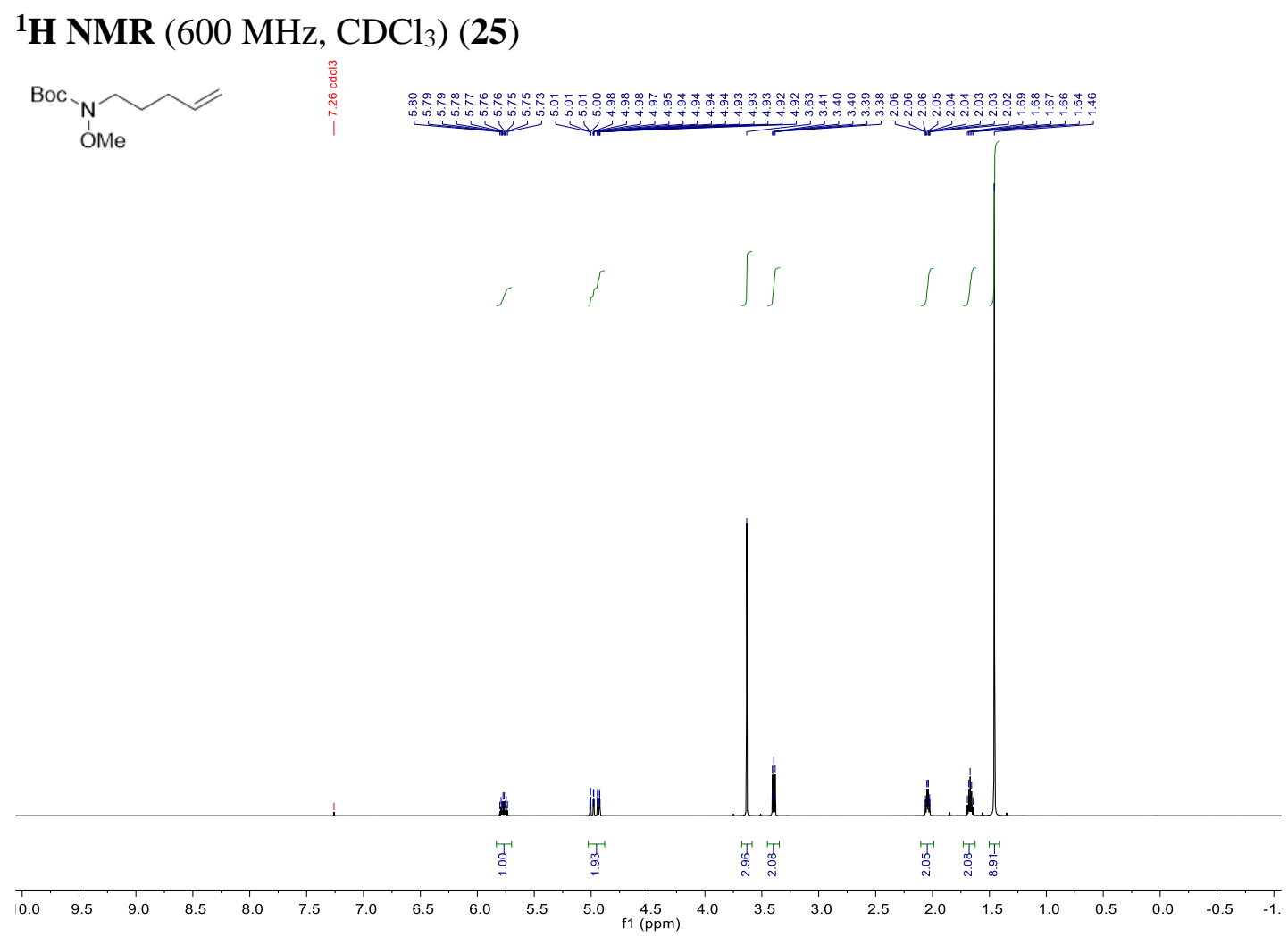

${ }^{13}$ C NMR (151 MHz, $\left.\mathrm{CDCl}_{3}\right)$ (25)
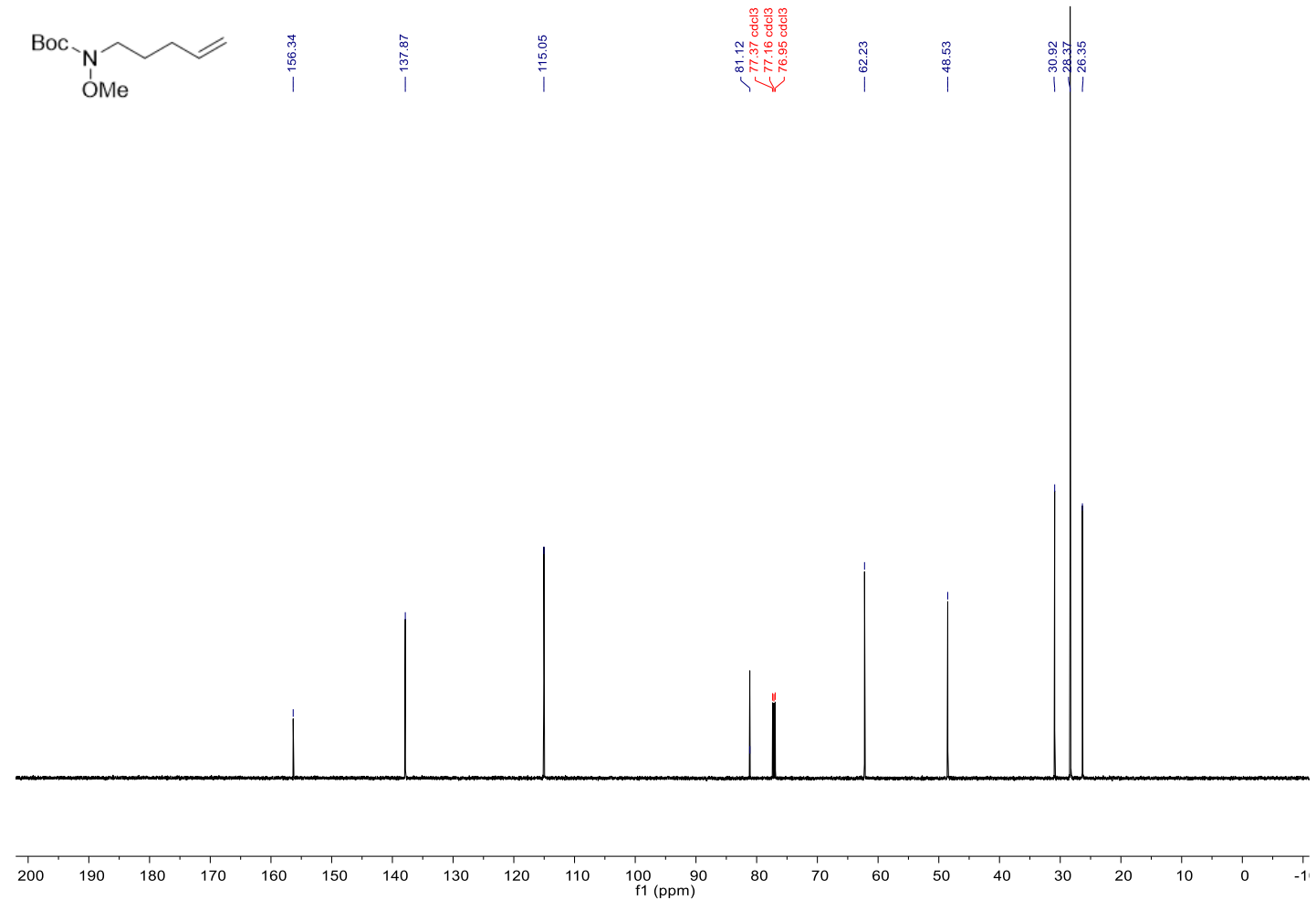


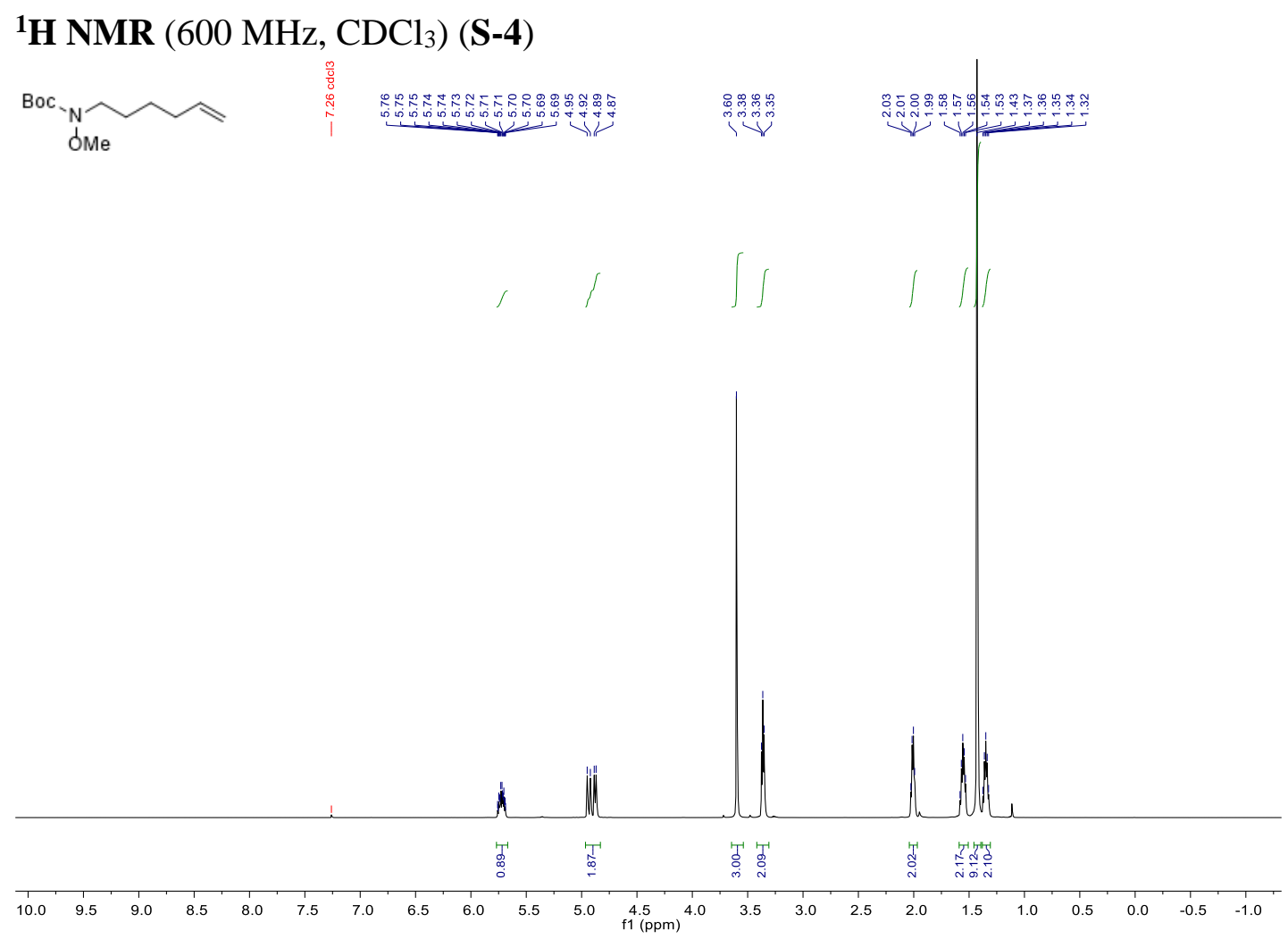

${ }^{13} \mathbf{C}$ NMR $\left(151 \mathrm{MHz}, \mathrm{CDCl}_{3}\right)(\mathbf{S}-\mathbf{4})$
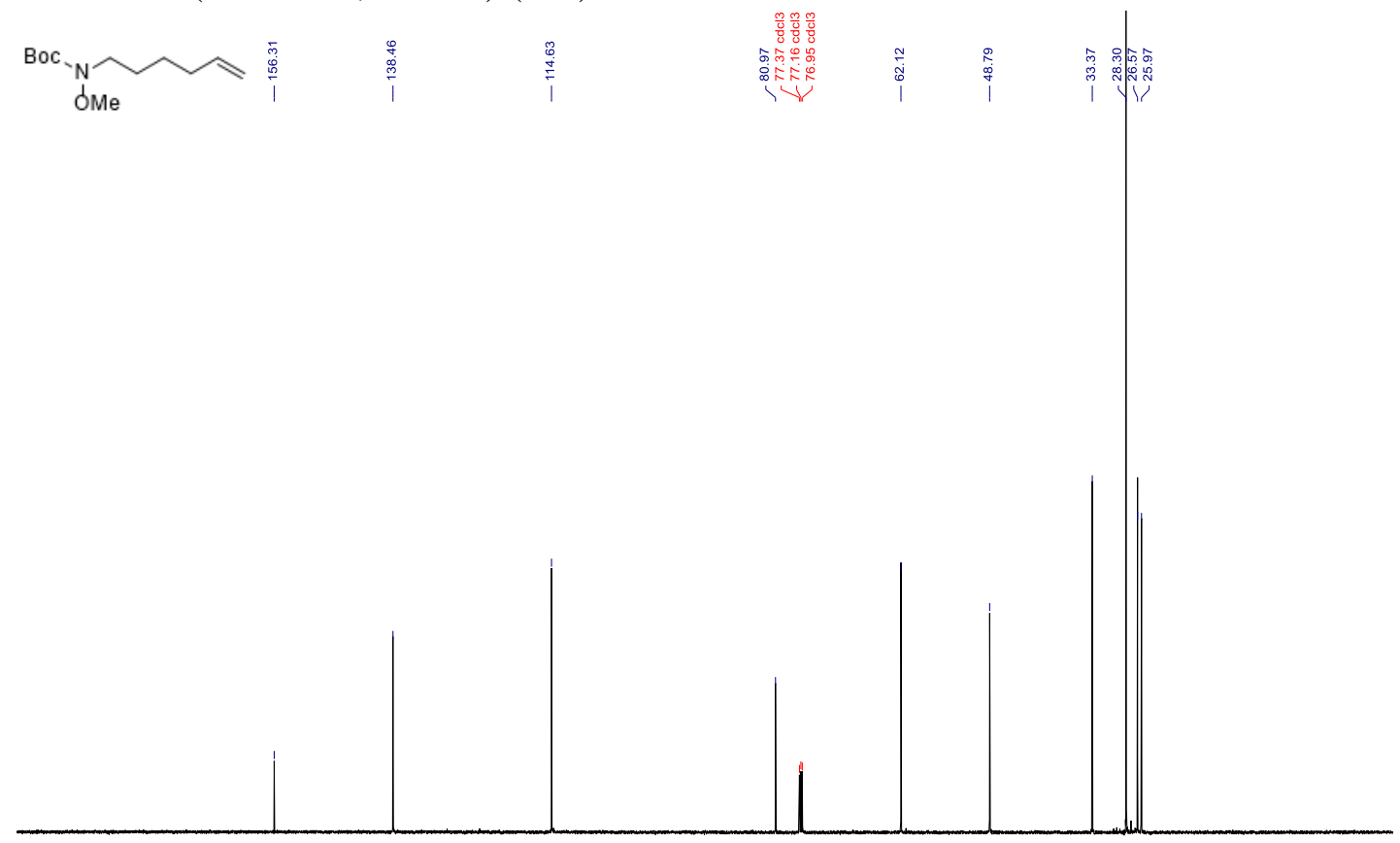

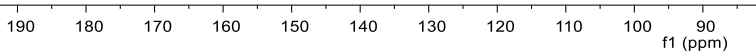




\section{${ }^{1} \mathbf{H}$ NMR (600 MHz, $\left.\mathrm{CDCl}_{3}\right)(\mathbf{S - 5})$}

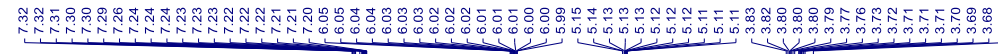
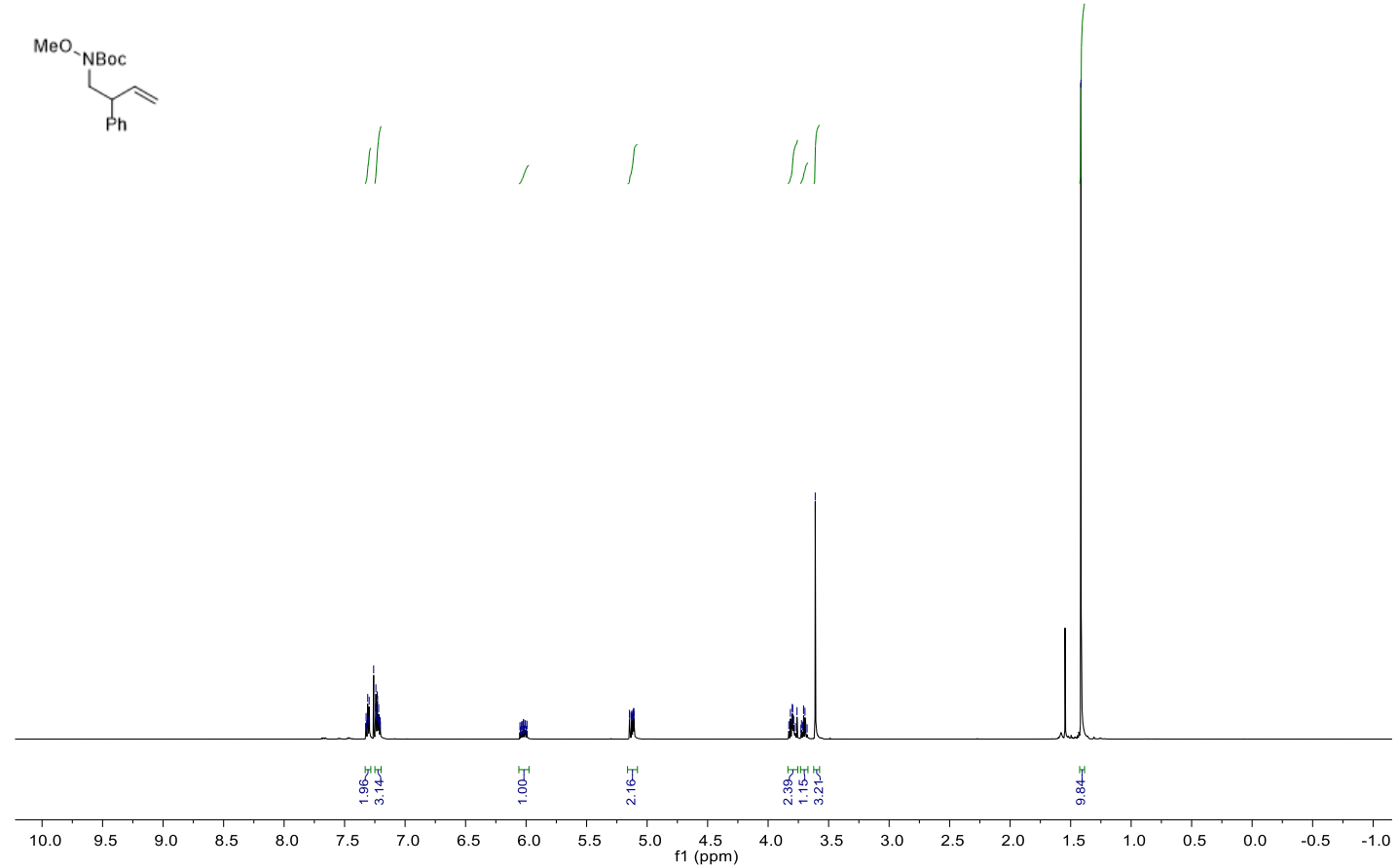

${ }^{13} \mathbf{C ~ N M R}\left(126 \mathrm{MHz}, \mathrm{CDCl}_{3}\right)(\mathbf{S}-5)$
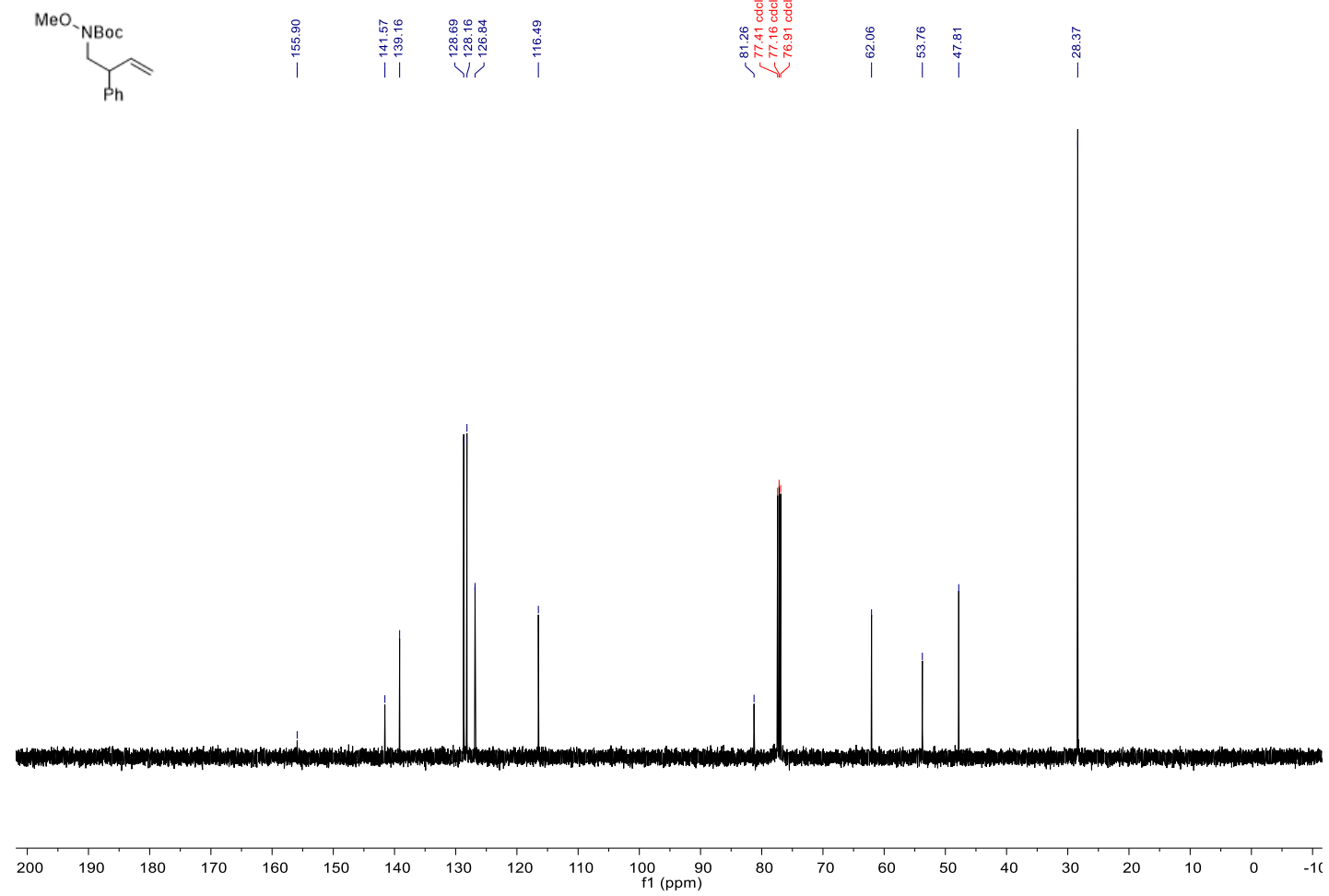
Xu, Zhang, Ingoglia, Allais, Dechert-Schmitt, Singer and Morken Supporting Information

${ }^{1} \mathbf{H}$ NMR $\left(600 \mathrm{MHz}, \mathrm{CDCl}_{3}\right)$ (S-6)

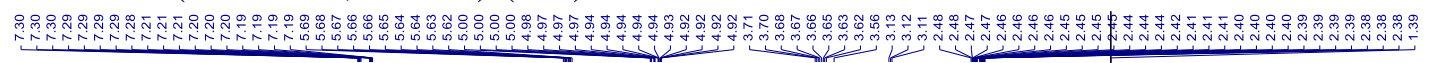
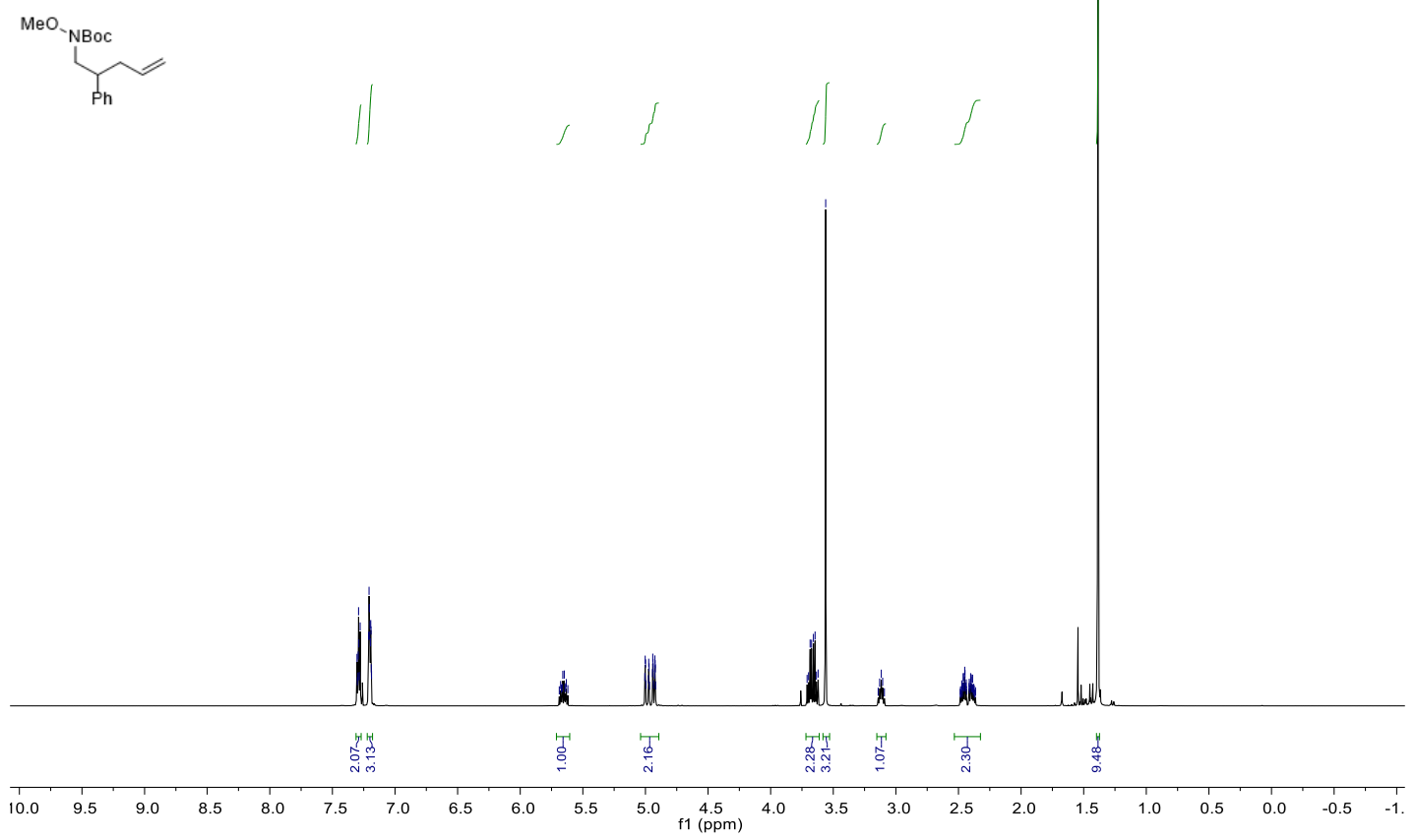

${ }^{13} \mathbf{C}$ NMR $\left(126 \mathrm{MHz}, \mathrm{CDCl}_{3}\right)(\mathbf{S}-\mathbf{6})$
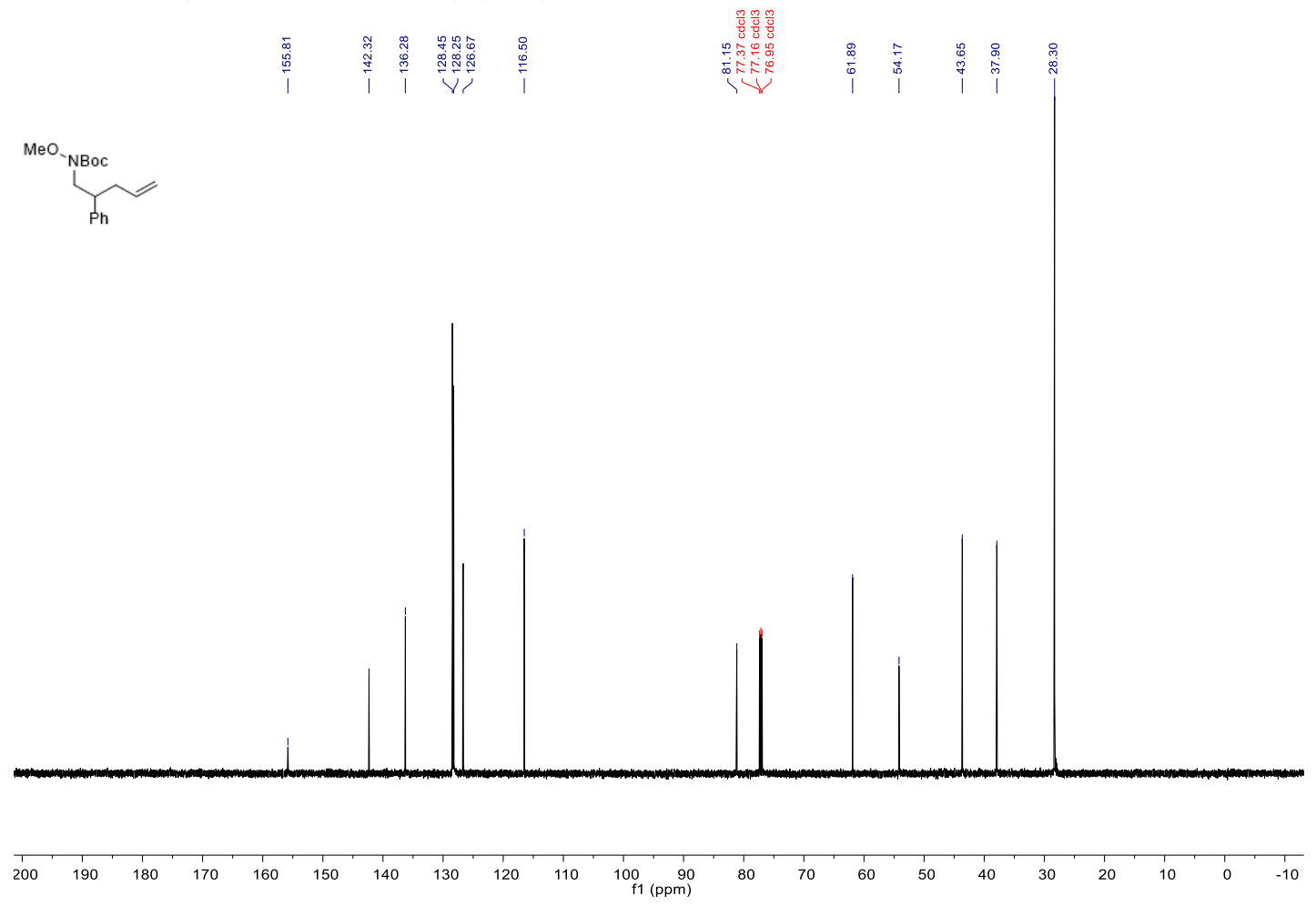

S-36 


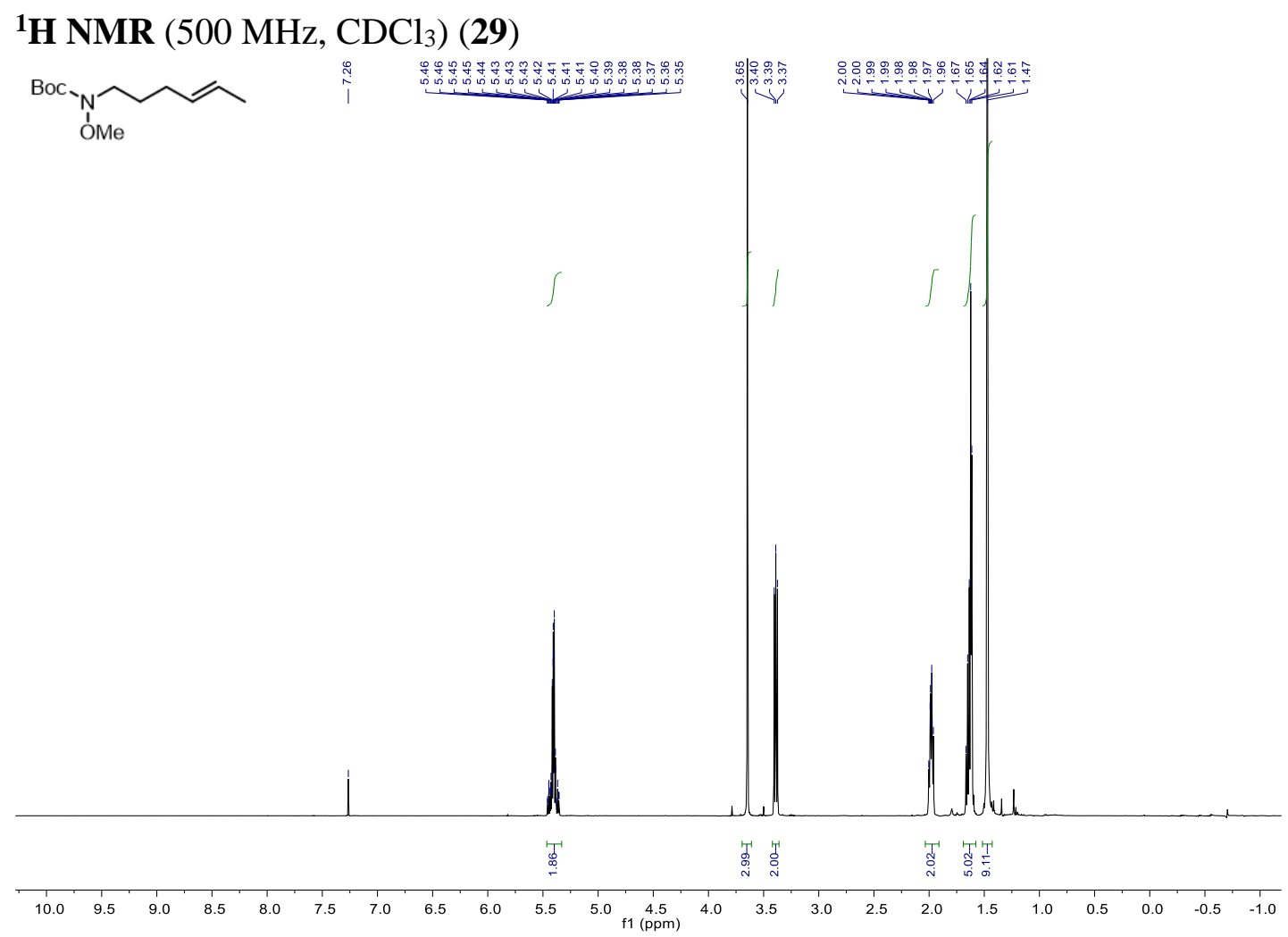

${ }^{13} \mathbf{C ~ N M R}\left(126 \mathrm{MHz}, \mathrm{CDCl}_{3}\right)(\mathbf{2 9})$
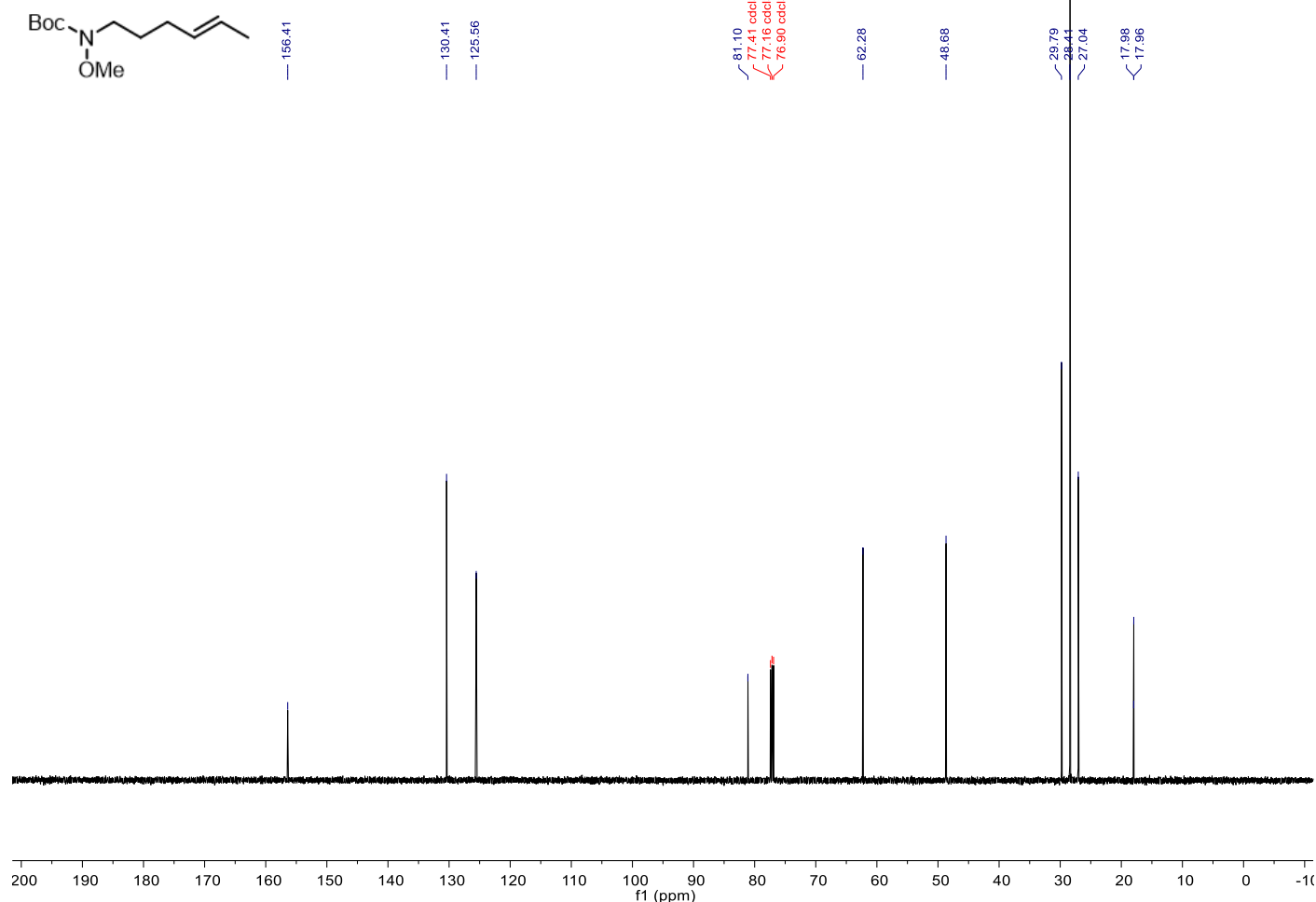


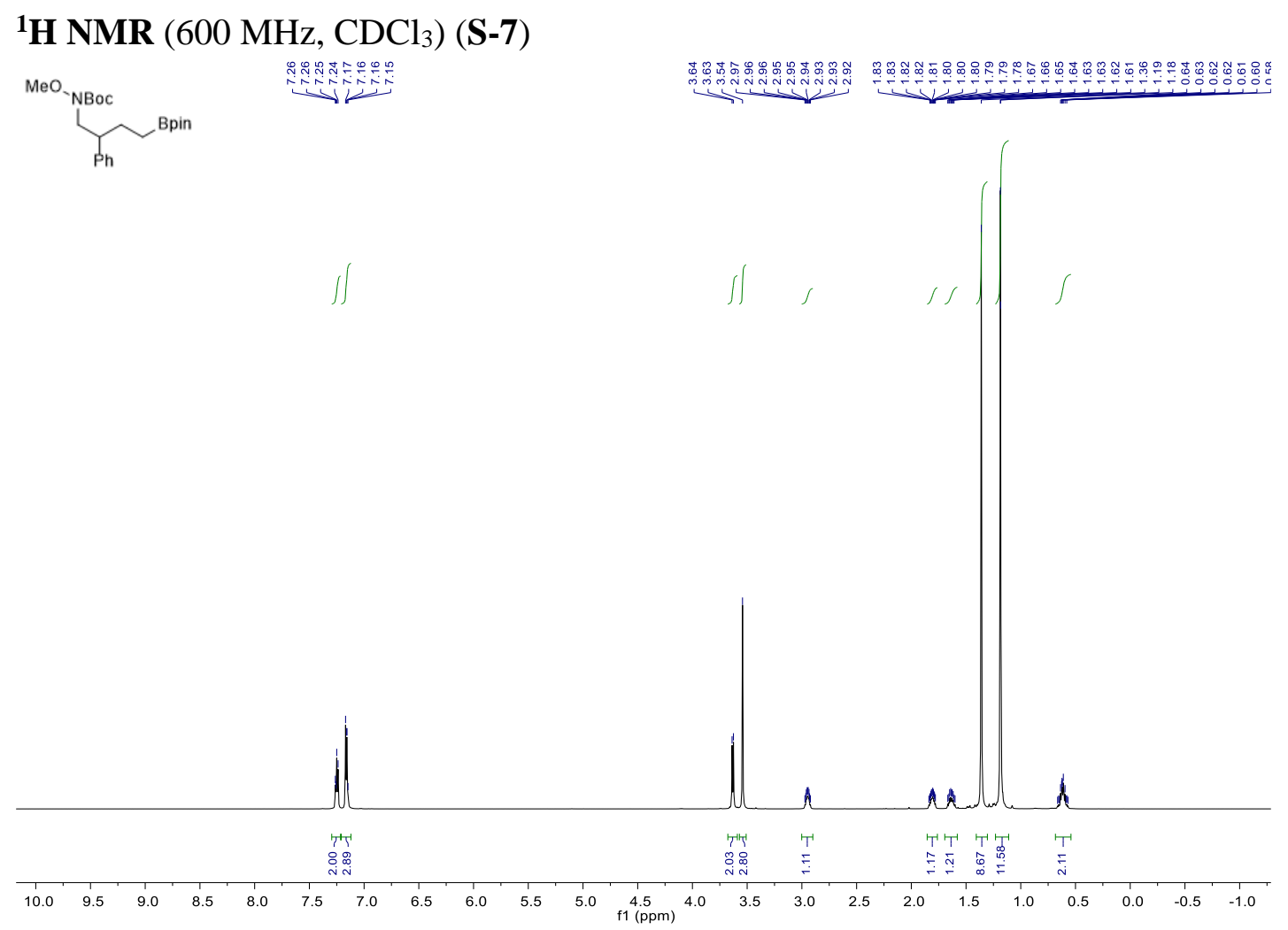

${ }^{13} \mathbf{C}$ NMR (126 MHz, $\left.\mathrm{CDCl}_{3}\right)(\mathbf{S}-\mathbf{7})$
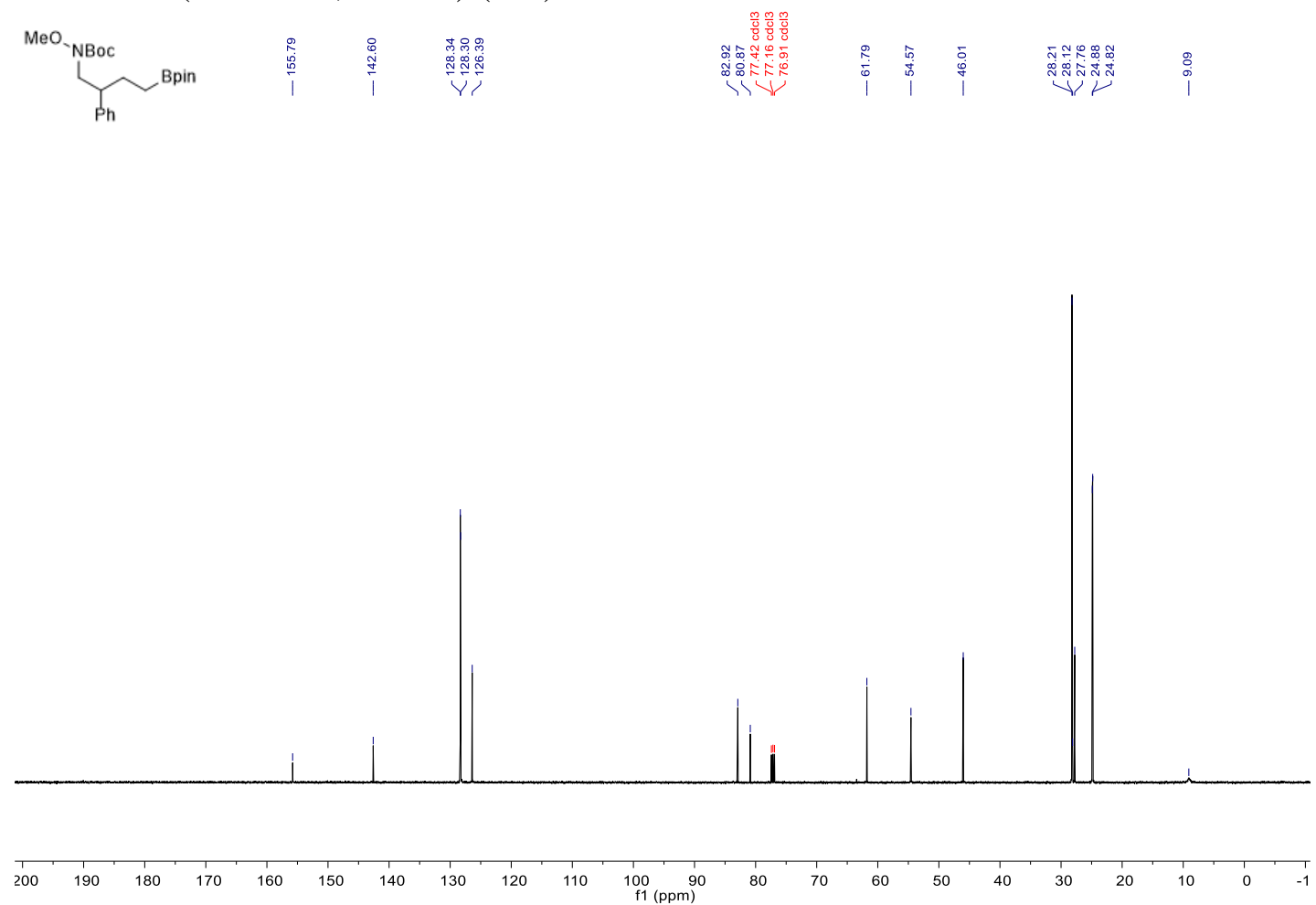


\section{${ }^{1} \mathbf{H} \mathbf{N M R}\left(600 \mathrm{MHz}, \mathrm{CDCl}_{3}\right)(\mathbf{S - 8})$}

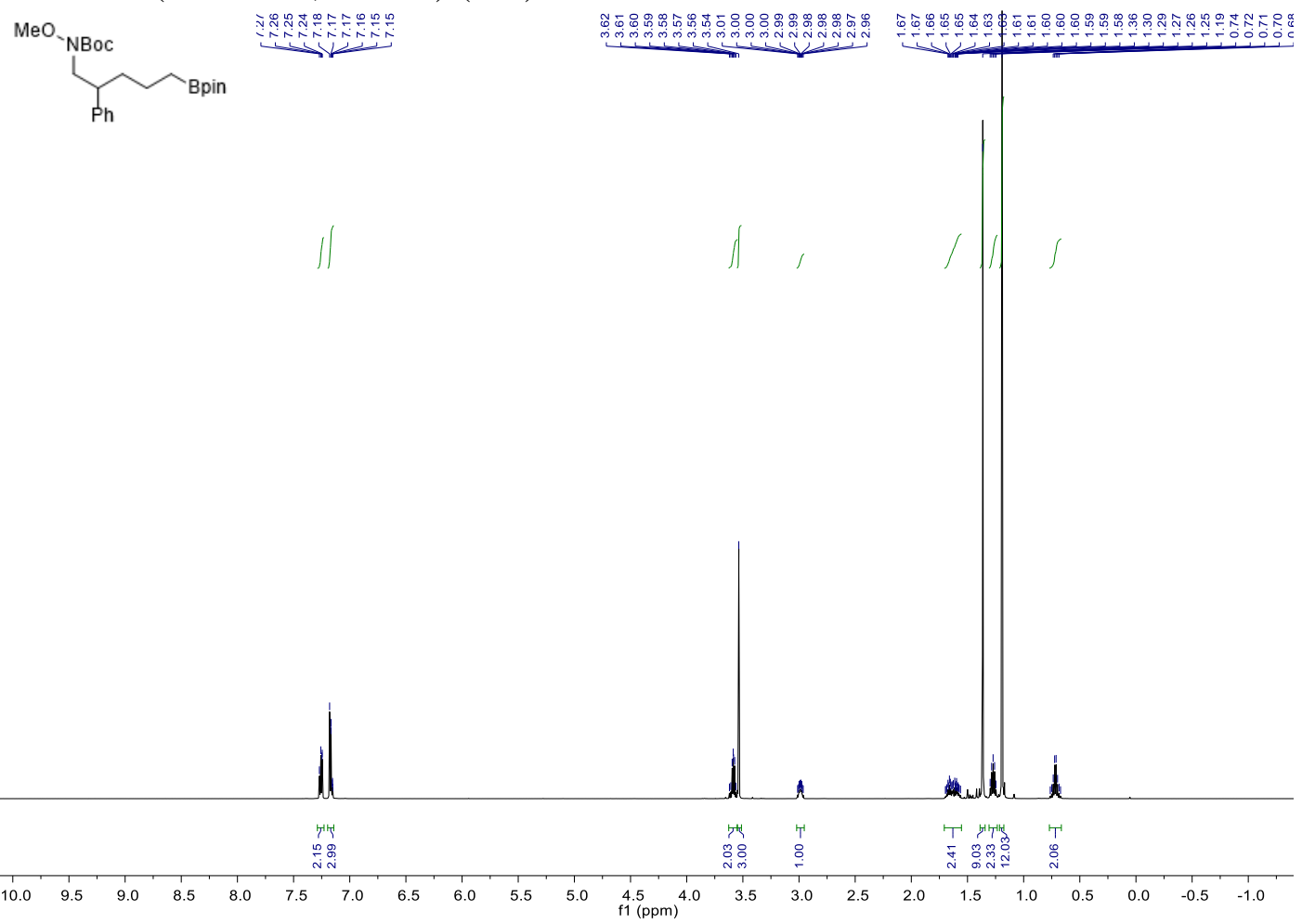

${ }^{13} \mathbf{C ~ N M R}\left(151 \mathrm{MHz}, \mathrm{CDCl}_{3}\right)(\mathbf{S - 8})$
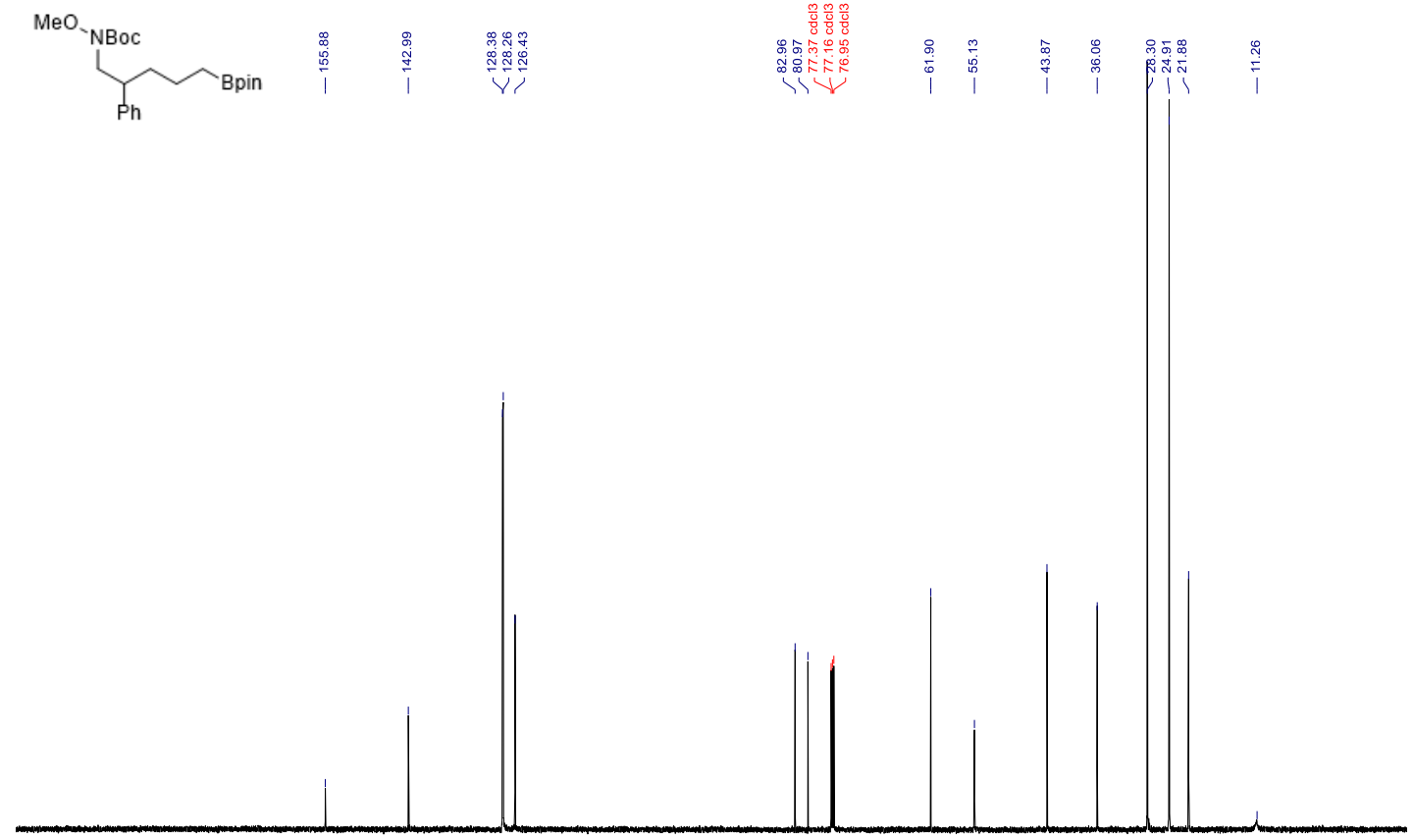

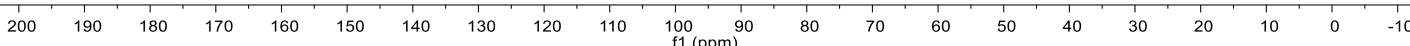




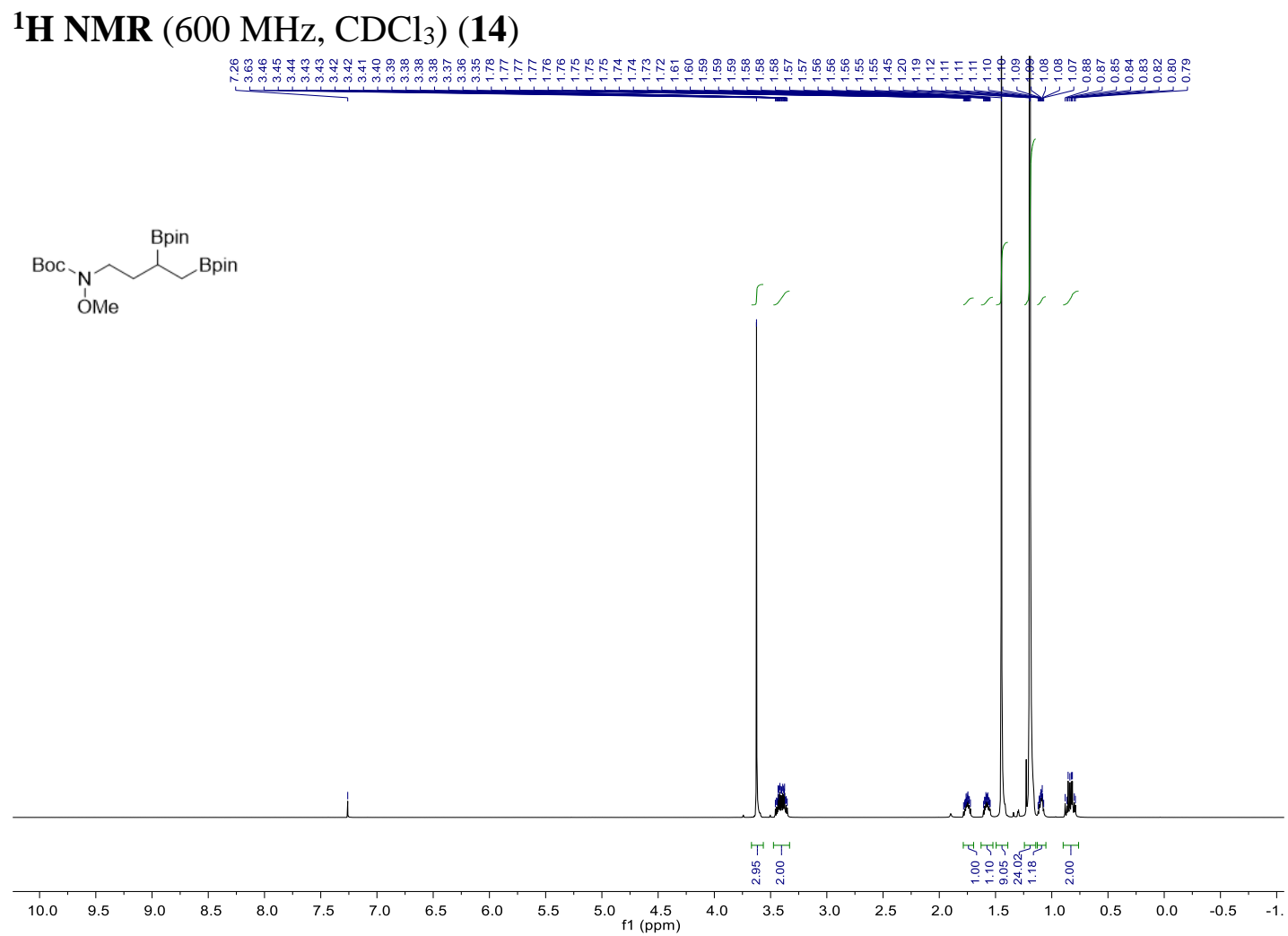

${ }^{13}$ C NMR (151 MHz, $\left.\mathrm{CDCl}_{3}\right)(\mathbf{1 4})$
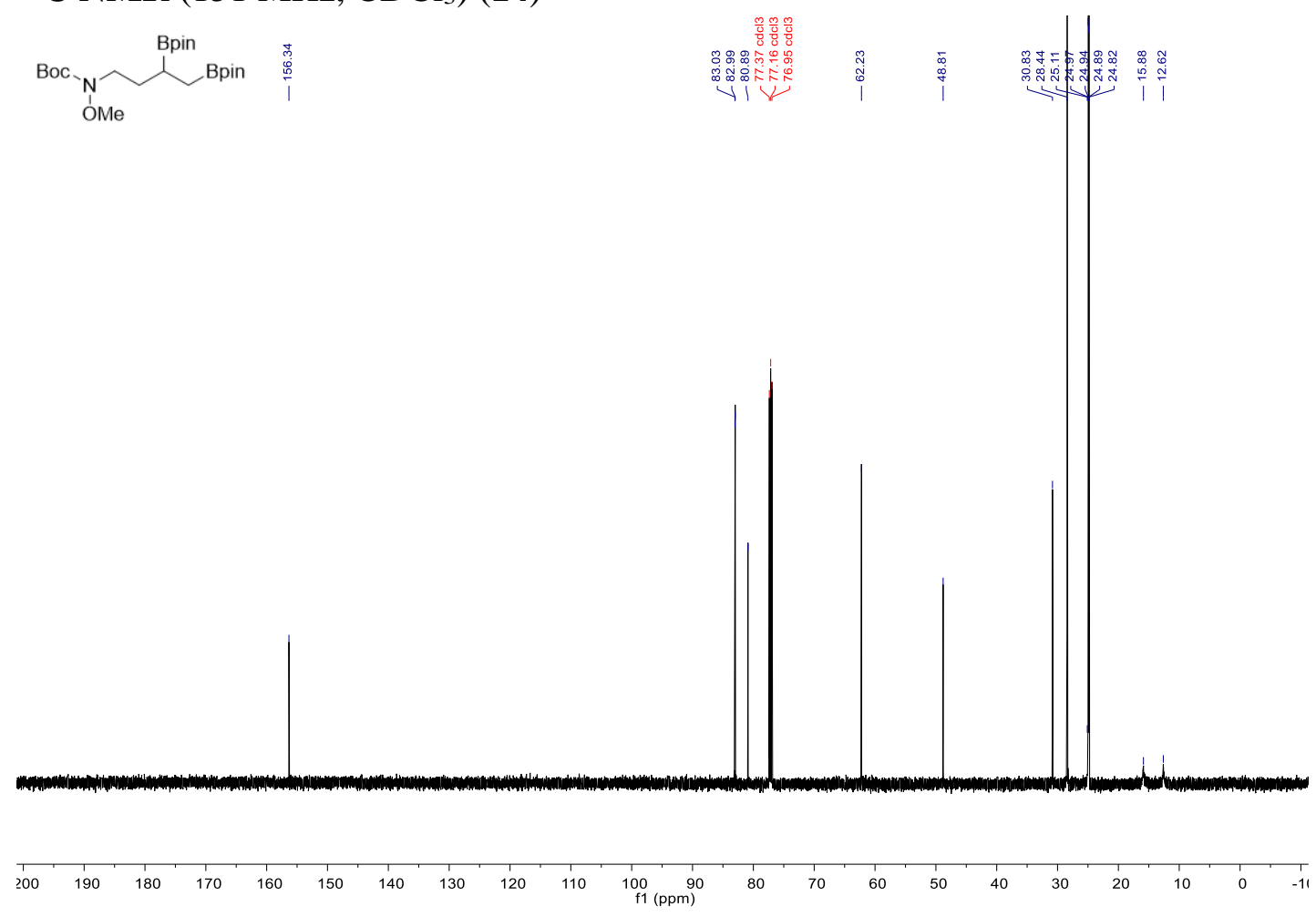


\section{${ }^{1} \mathbf{H}$ NMR (600 MHz, $\left.\mathrm{CDCl}_{3}\right)(\mathbf{1 6})$}

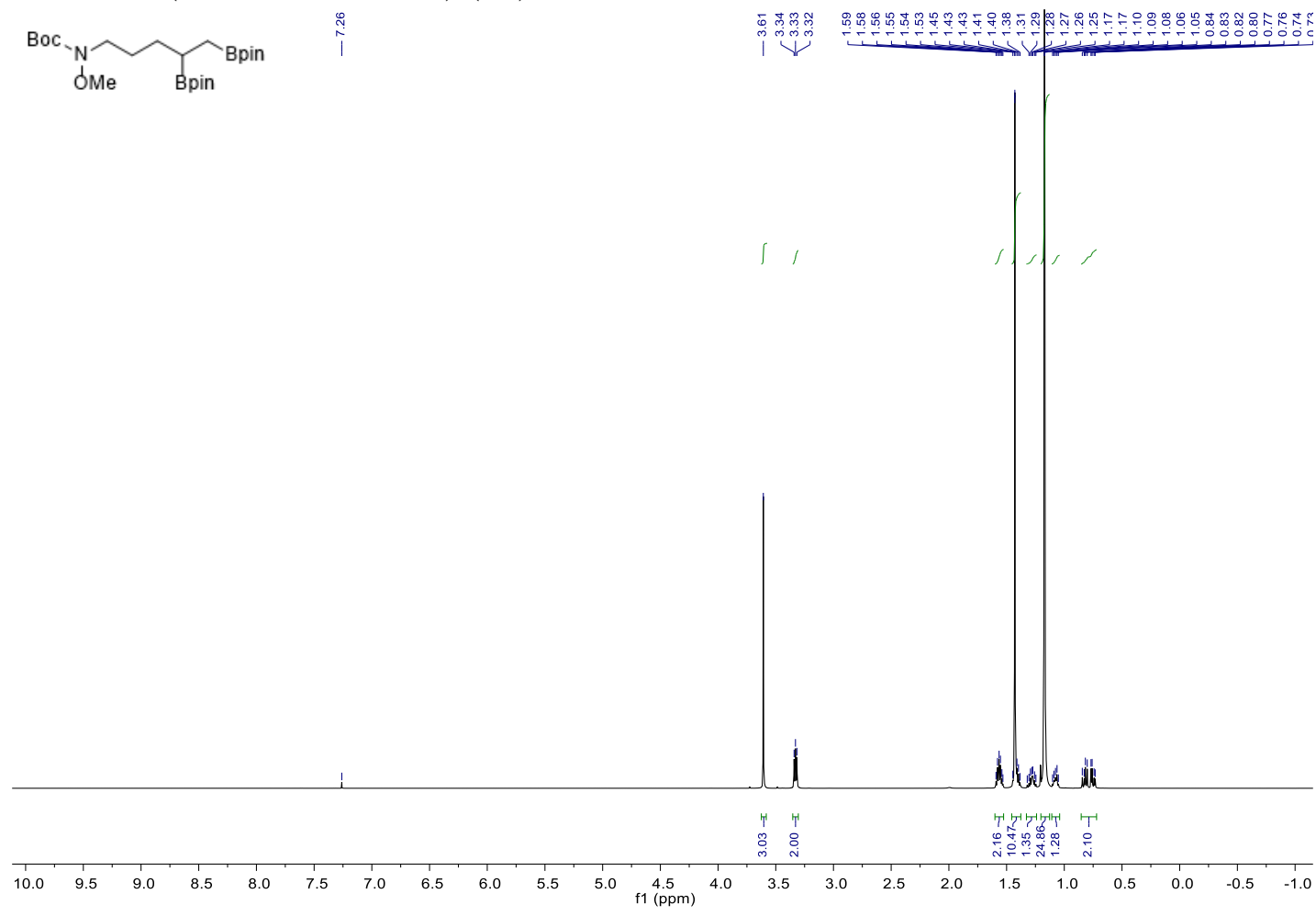

${ }^{13} \mathbf{C ~ N M R}\left(151 \mathrm{MHz}, \mathrm{CDCl}_{3}\right)(\mathbf{1 6})$
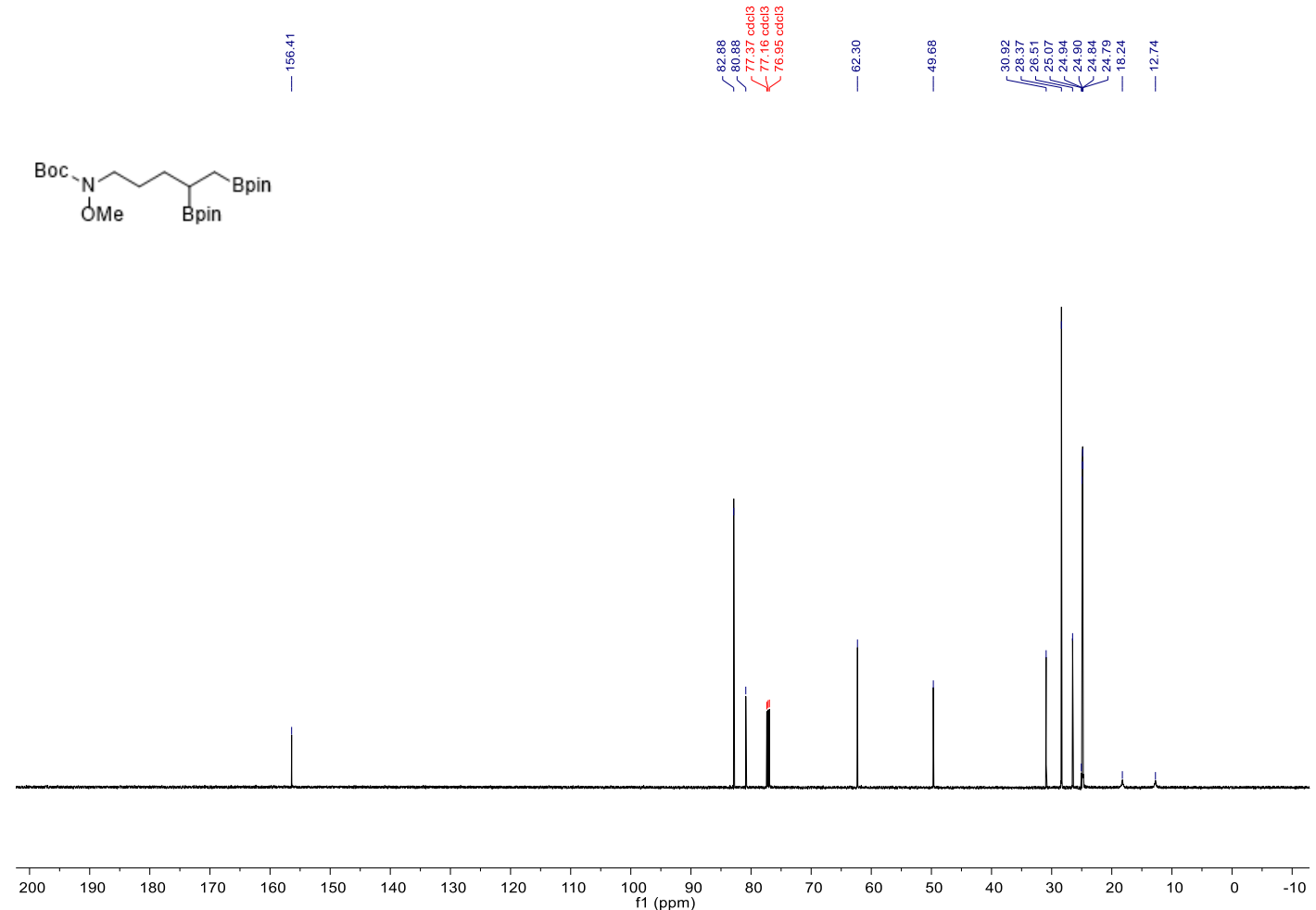


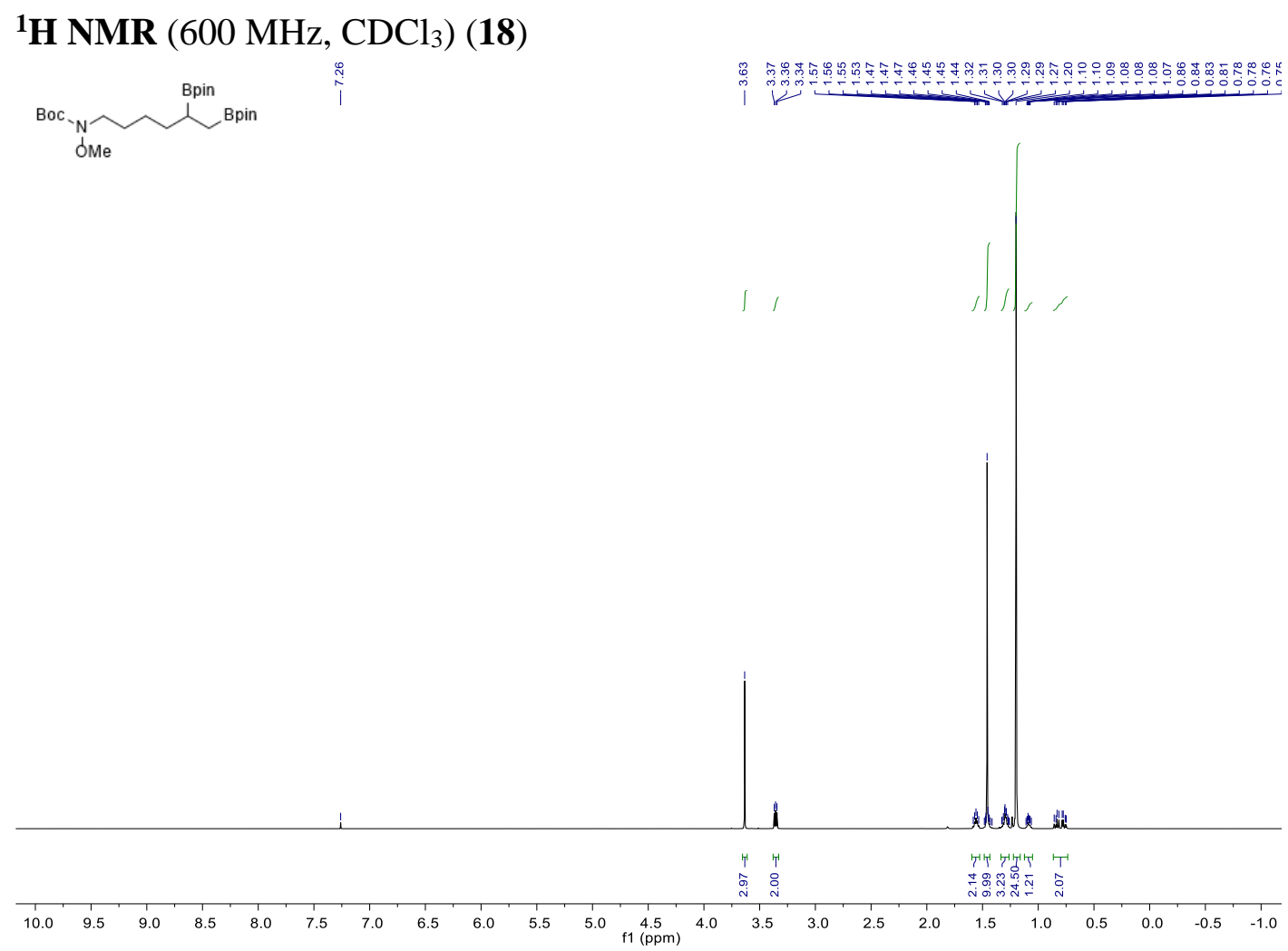

${ }^{13}$ C NMR (151 MHz, $\left.\mathrm{CDCl}_{3}\right)$ (18)
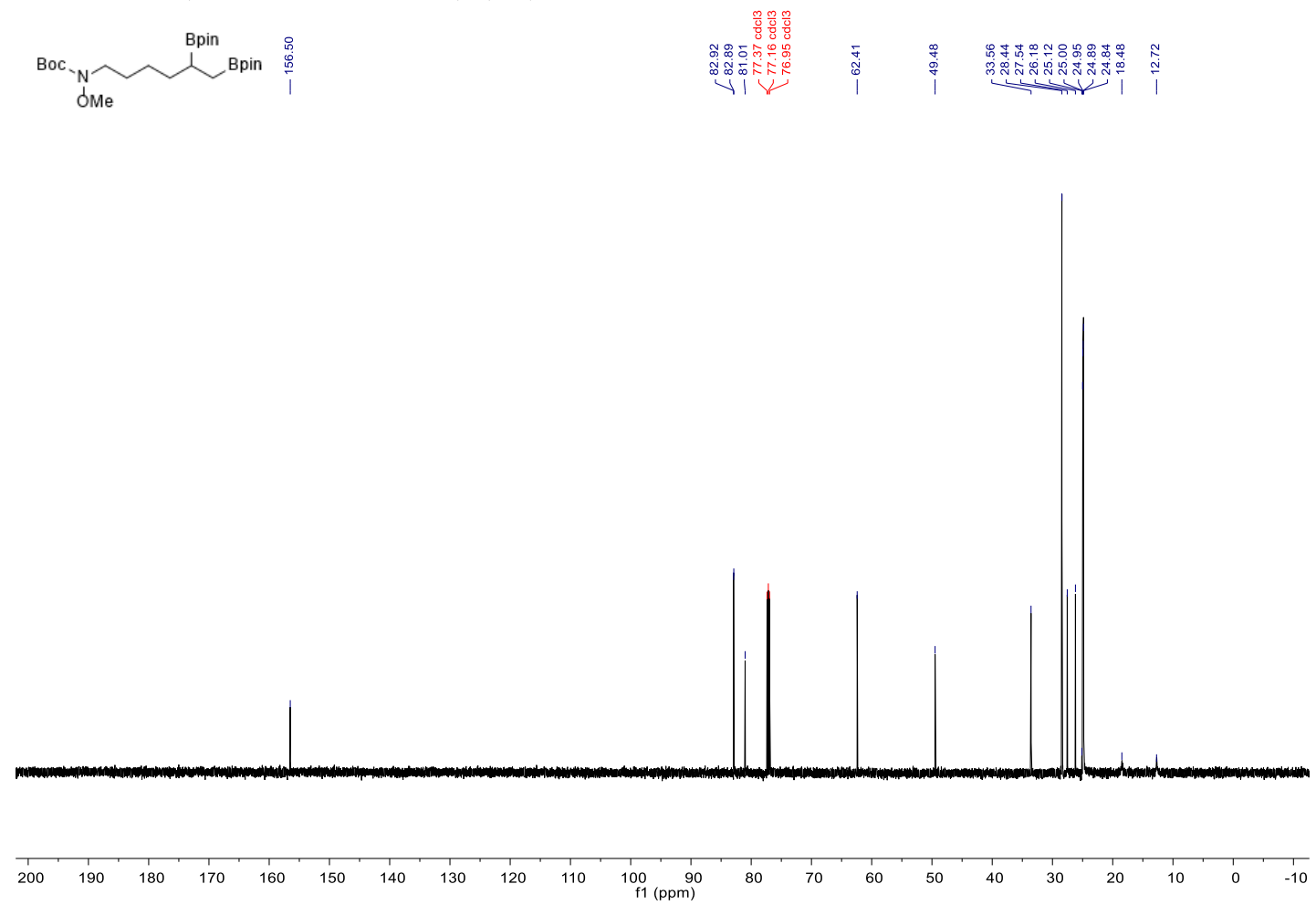


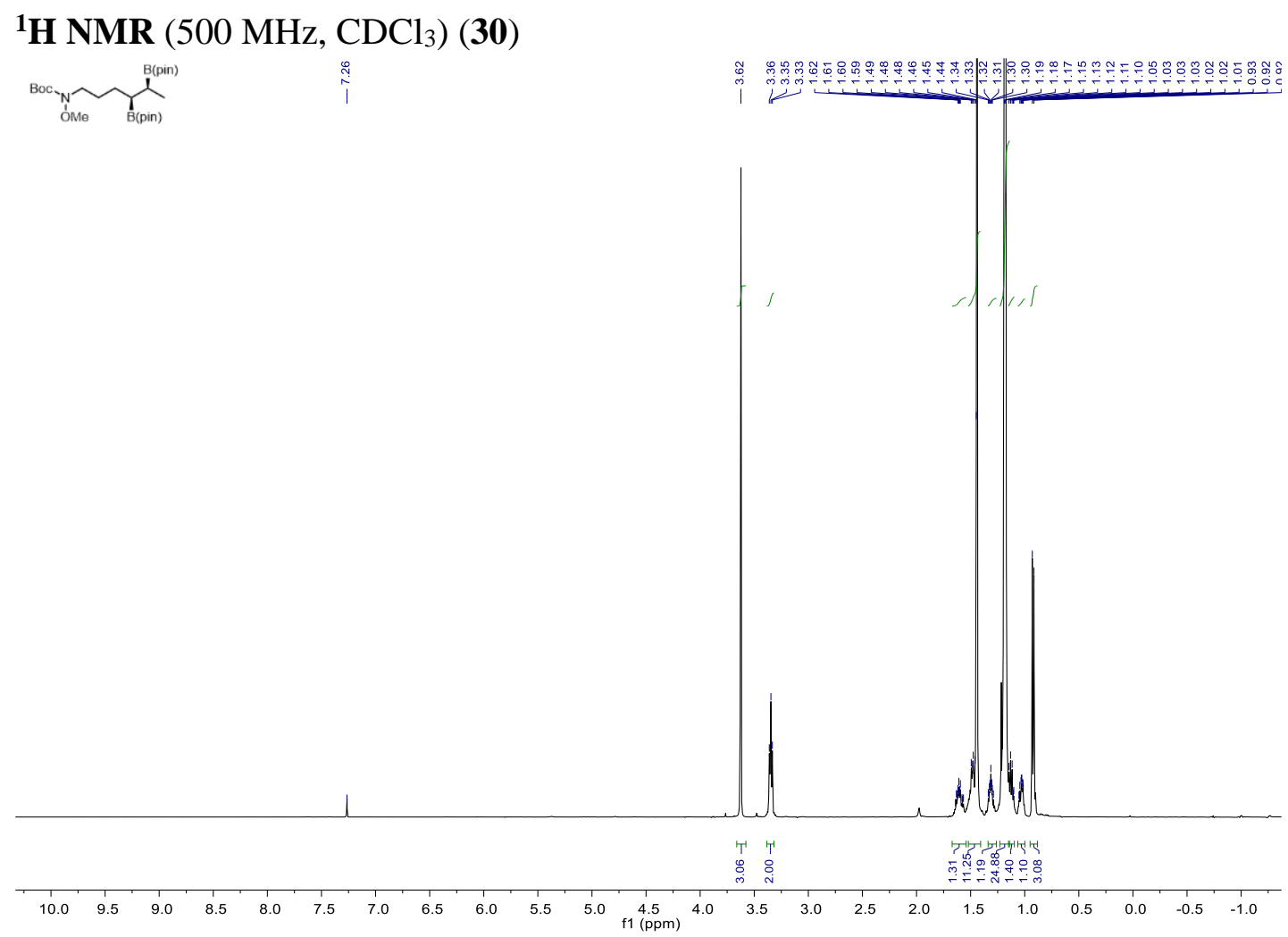

${ }^{13} \mathbf{C ~ N M R}\left(126 \mathrm{MHz}, \mathrm{CDCl}_{3}\right)(\mathbf{3 0})$
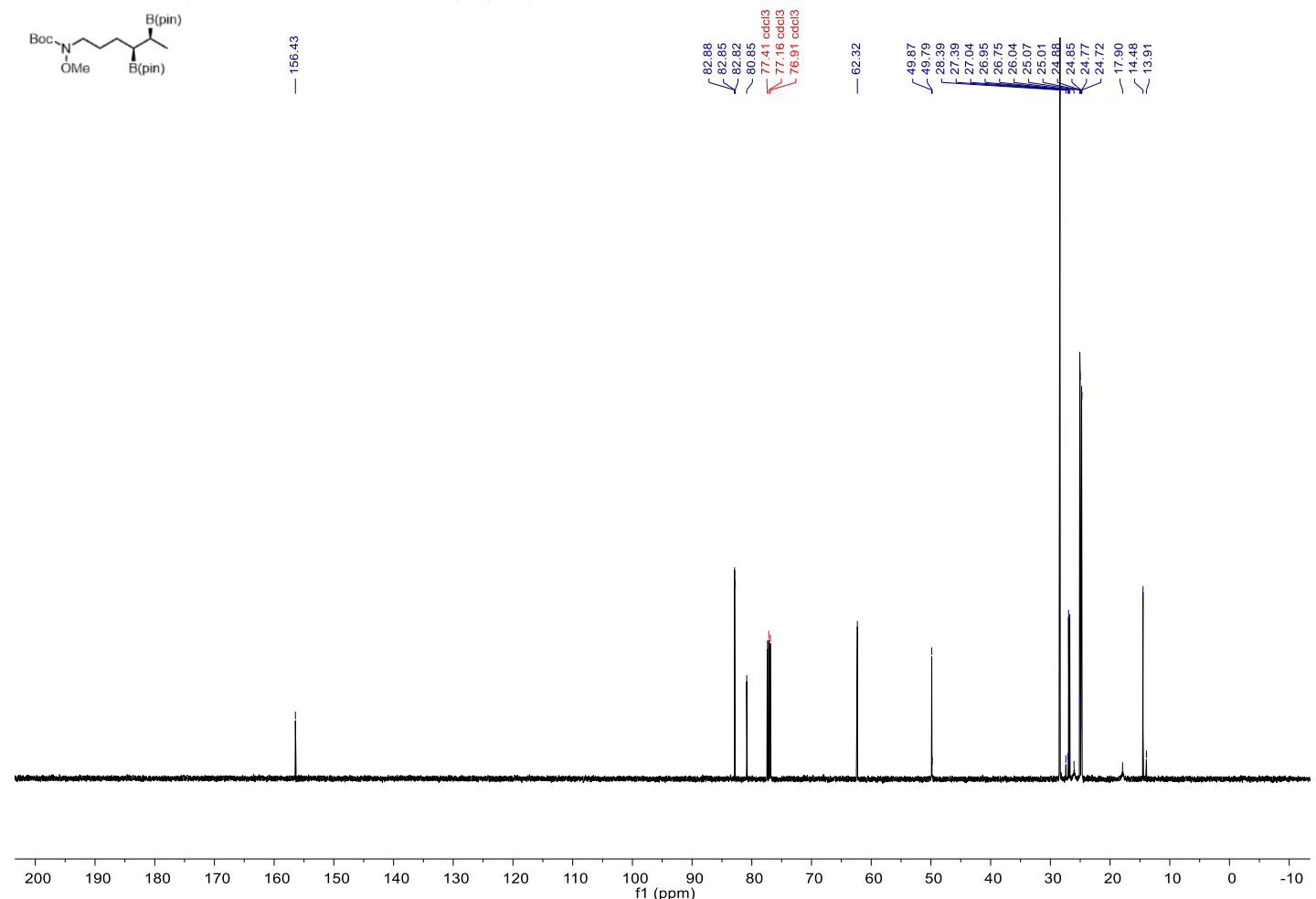


\section{${ }^{\mathbf{1}} \mathbf{H}$ NMR $\left(600 \mathrm{MHz}, \mathrm{CDCl}_{3}\right)(\mathbf{S - 9})$}

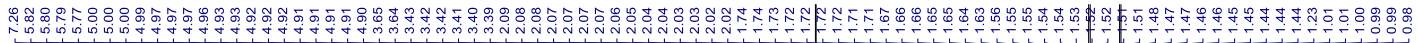
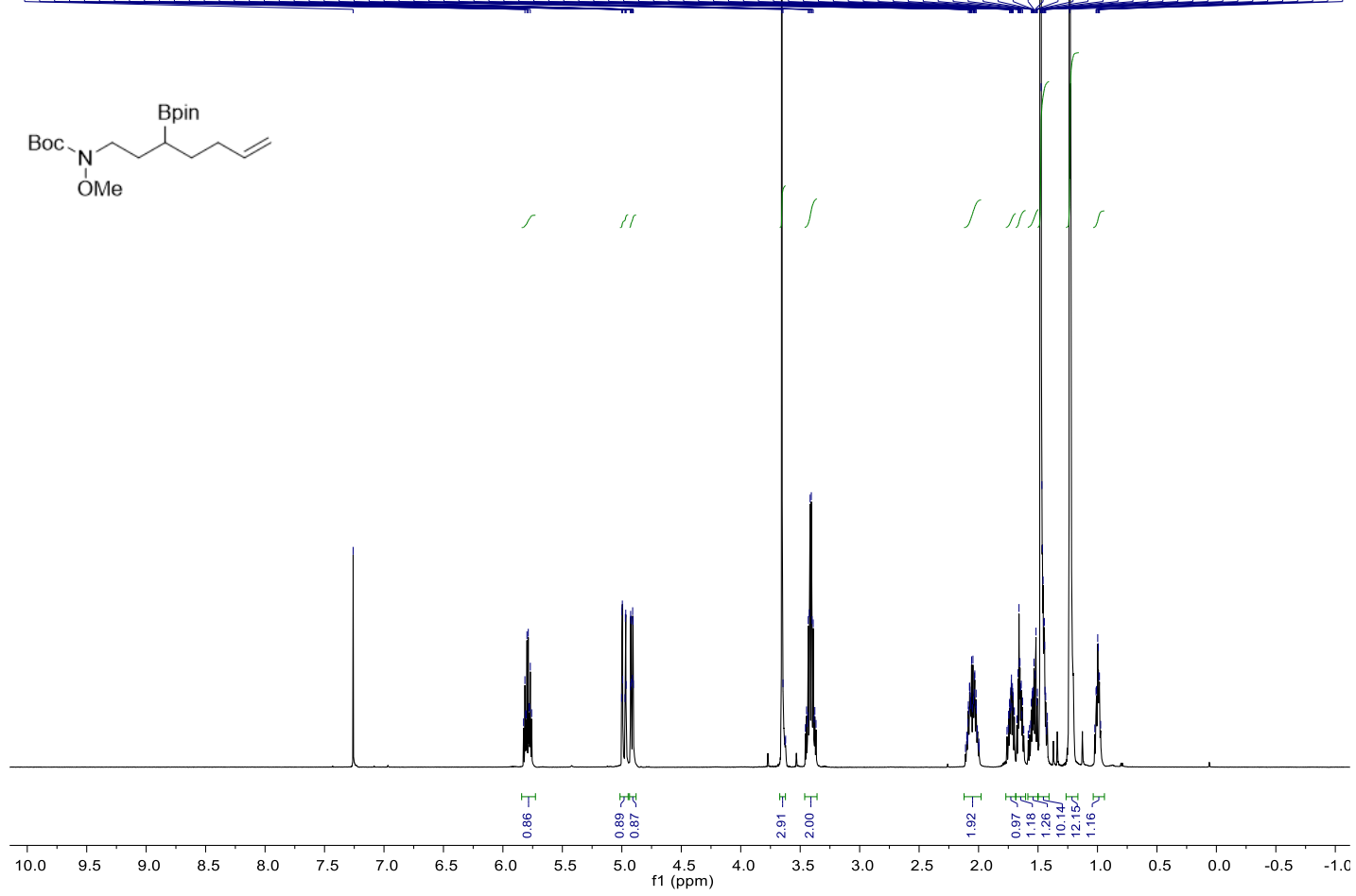

${ }^{13} \mathbf{C}$ NMR (126 MHz, $\left.\mathrm{CDCl}_{3}\right)(\mathbf{S - 9})$
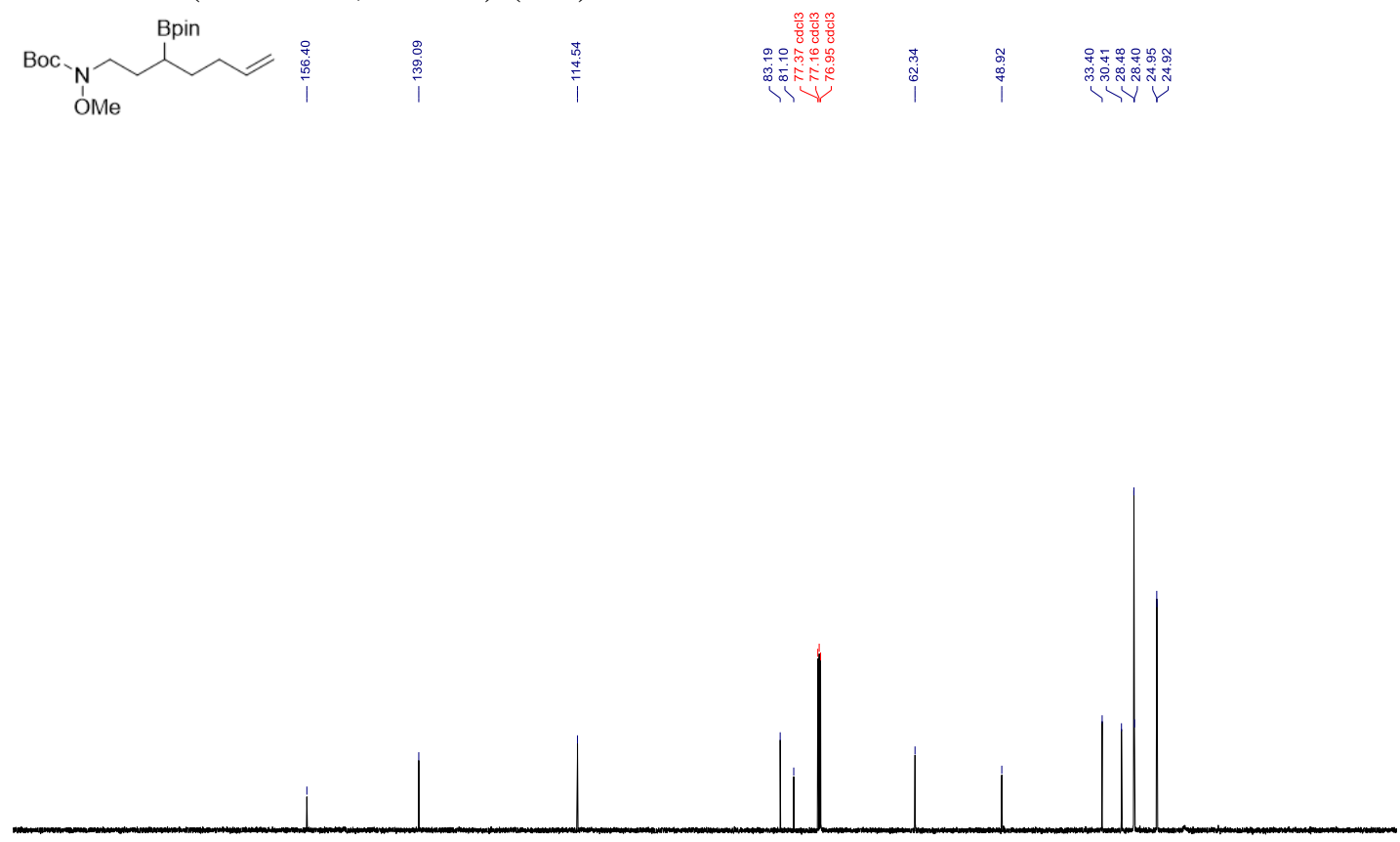

200

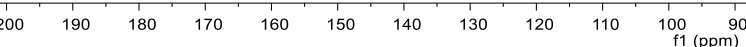


${ }^{1} \mathbf{H}$ NMR $\left(500 \mathrm{MHz}, \mathrm{CDCl}_{3}\right)(\mathbf{S - 1 0})$

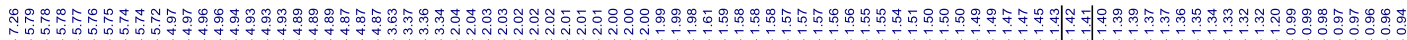

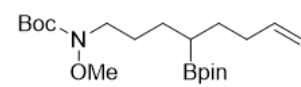

OMe Bpin
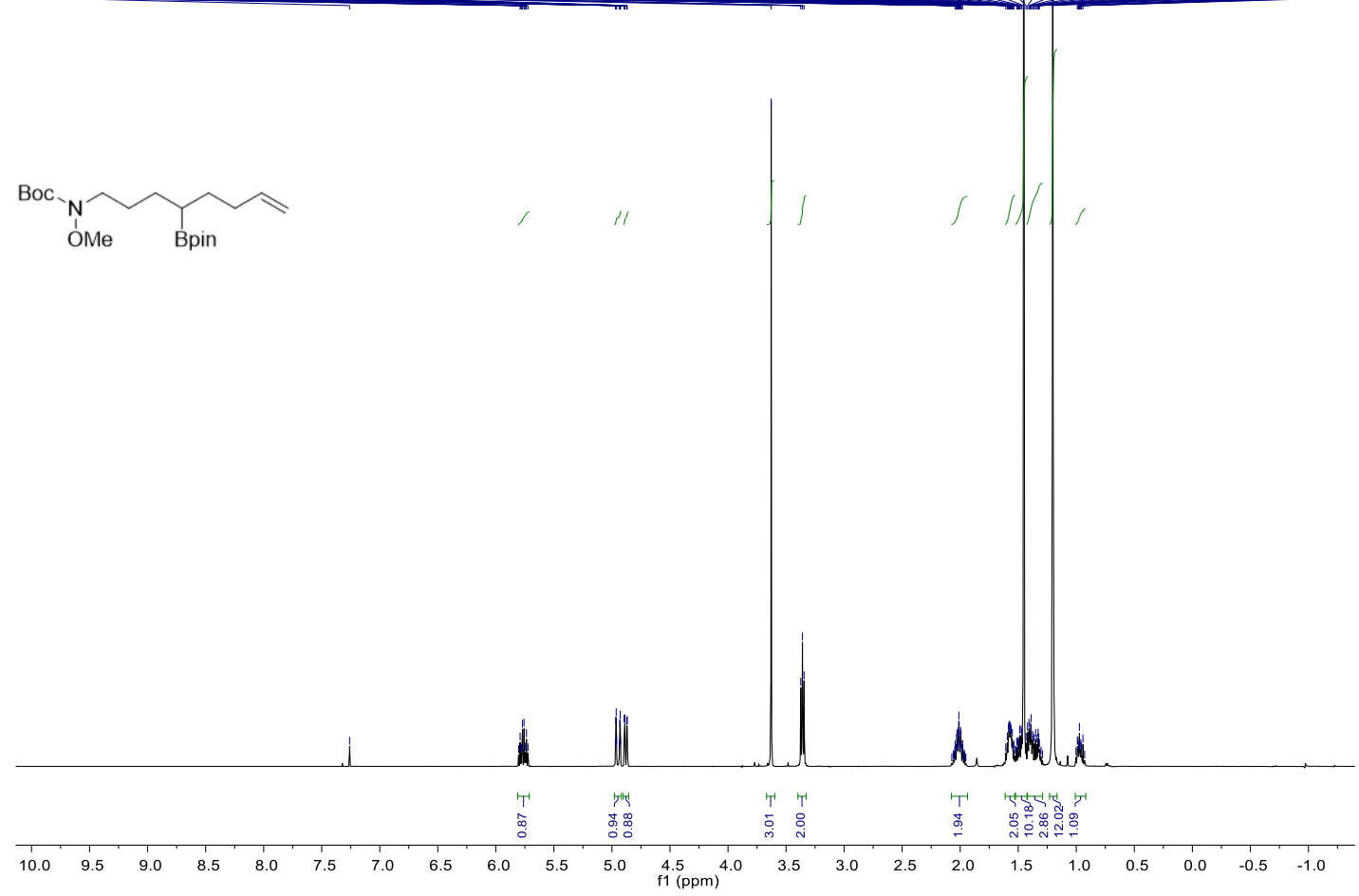

${ }^{13} \mathbf{C} \mathbf{N M R}\left(126 \mathrm{MHz}, \mathrm{CDCl}_{3}\right)(\mathbf{S}-\mathbf{1 0})$
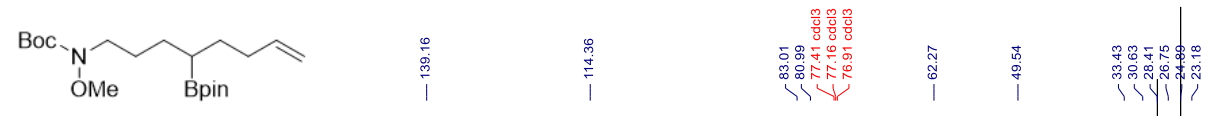

200

$\begin{array}{llllll}190 & 180 & 170 & 160 & 150\end{array}$
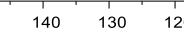

10090

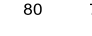




\section{${ }^{1} \mathbf{H}$ NMR (600 MHz, $\left.\mathrm{CDCl}_{3}\right)$ (S-11)}

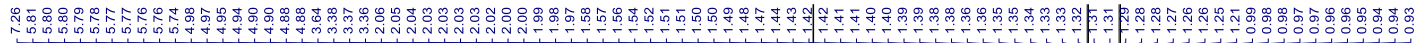
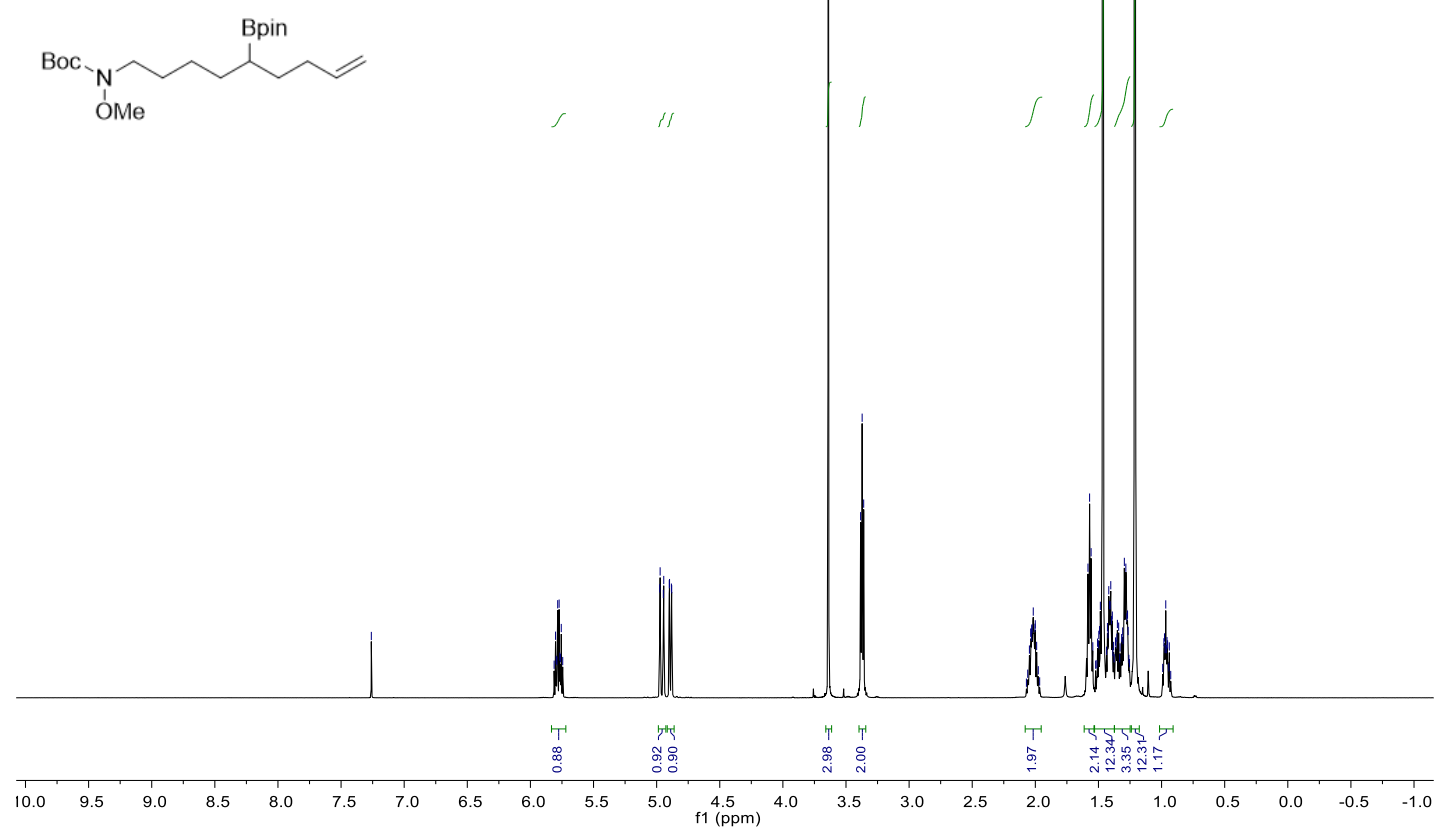

${ }^{13} \mathbf{C} \mathbf{N M R}\left(151 \mathrm{MHz}, \mathrm{CDCl}_{3}\right)(\mathbf{S}-\mathbf{1 1})$
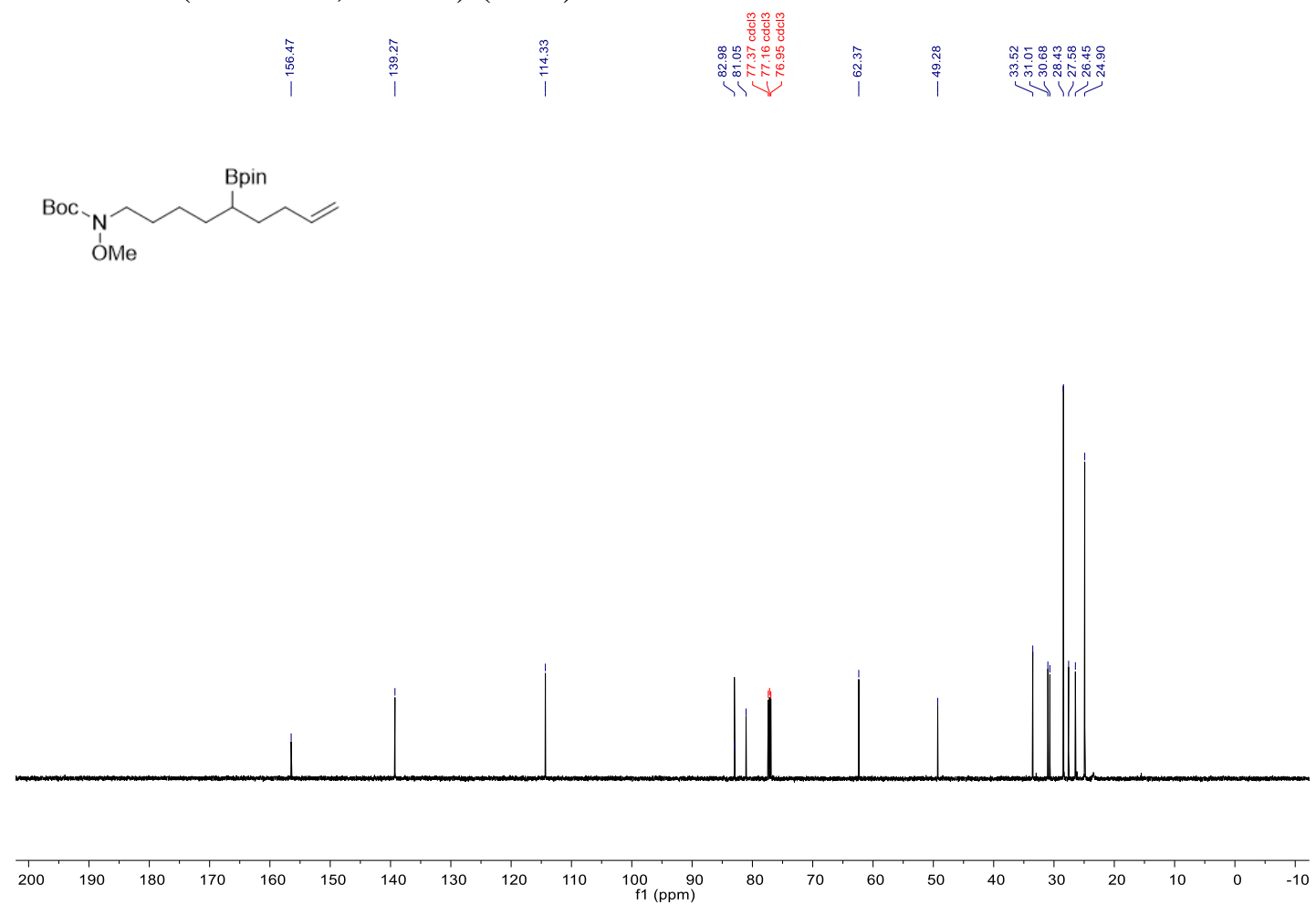
${ }^{1}$ H NMR $\left(500 \mathrm{MHz}, \mathrm{CDCl}_{3}\right)(\mathbf{S - 1 2})$

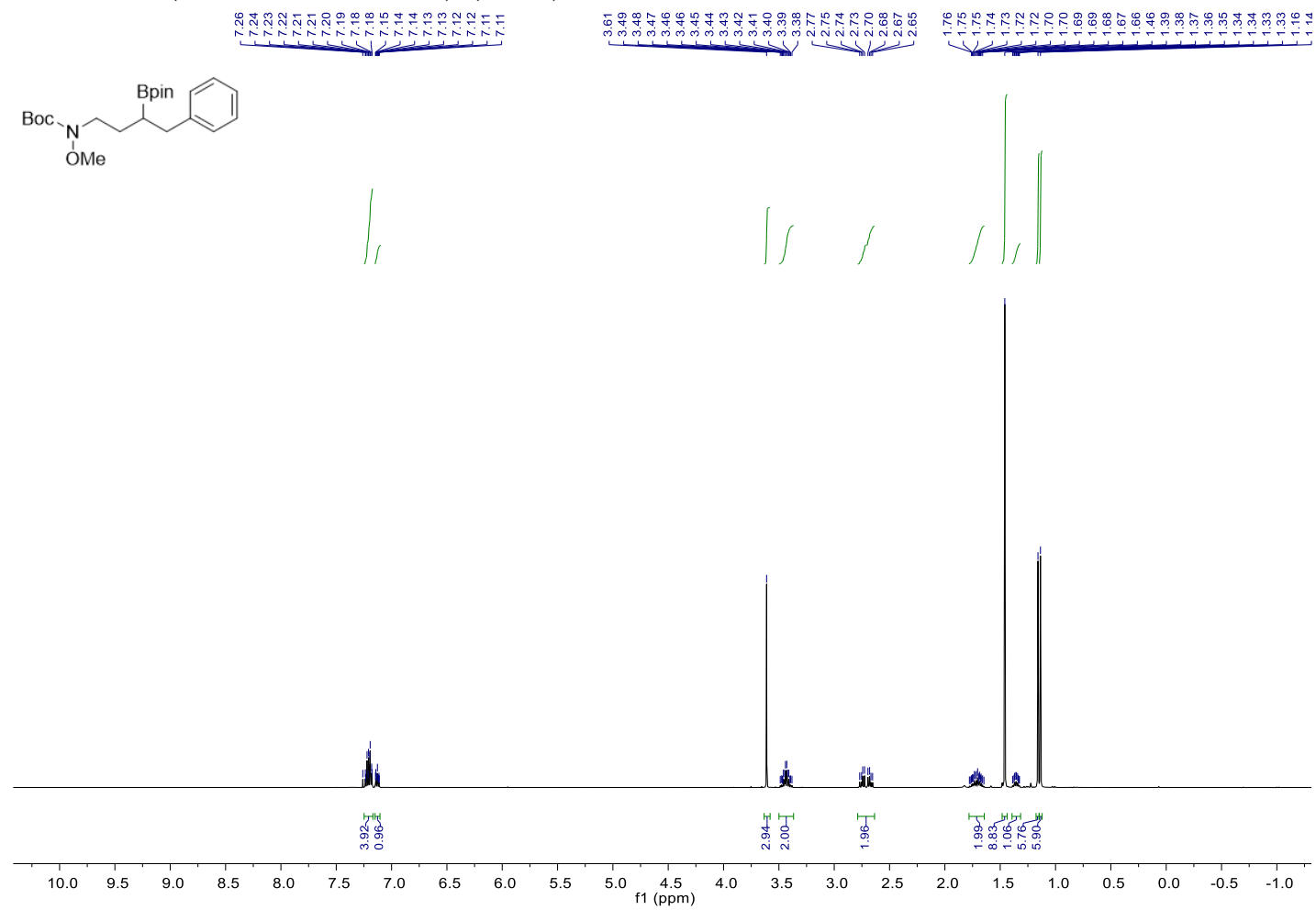

${ }^{13} \mathbf{C}$ NMR (126 MHz, $\left.\mathrm{CDCl}_{3}\right)$ (S-12)
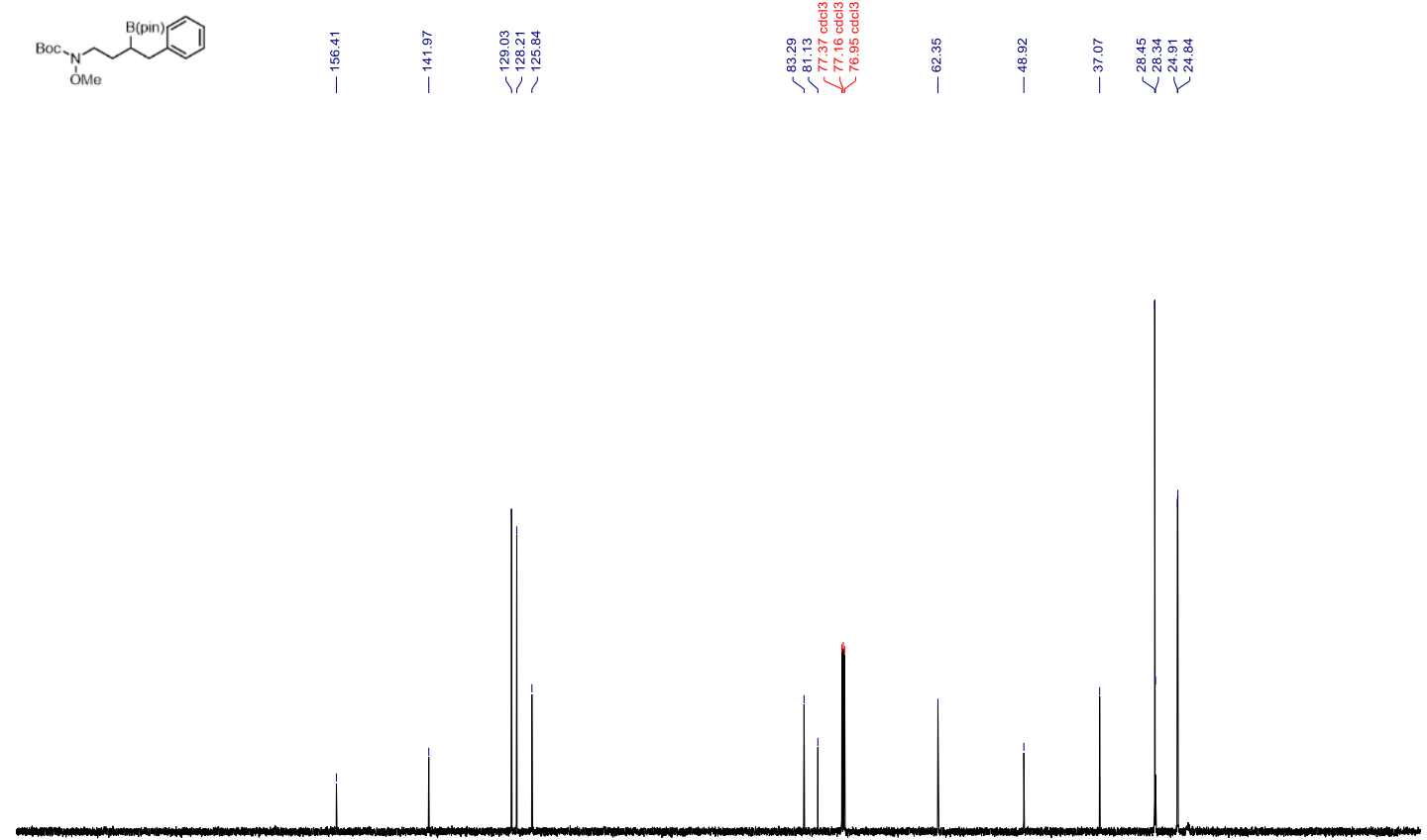

200

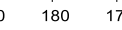

$160 \quad 150$

140

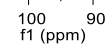




\section{${ }^{1} \mathbf{H}$ NMR $\left(600 \mathrm{MHz}, \mathrm{CDCl}_{3}\right)(\mathbf{S}-\mathbf{1 3})$}

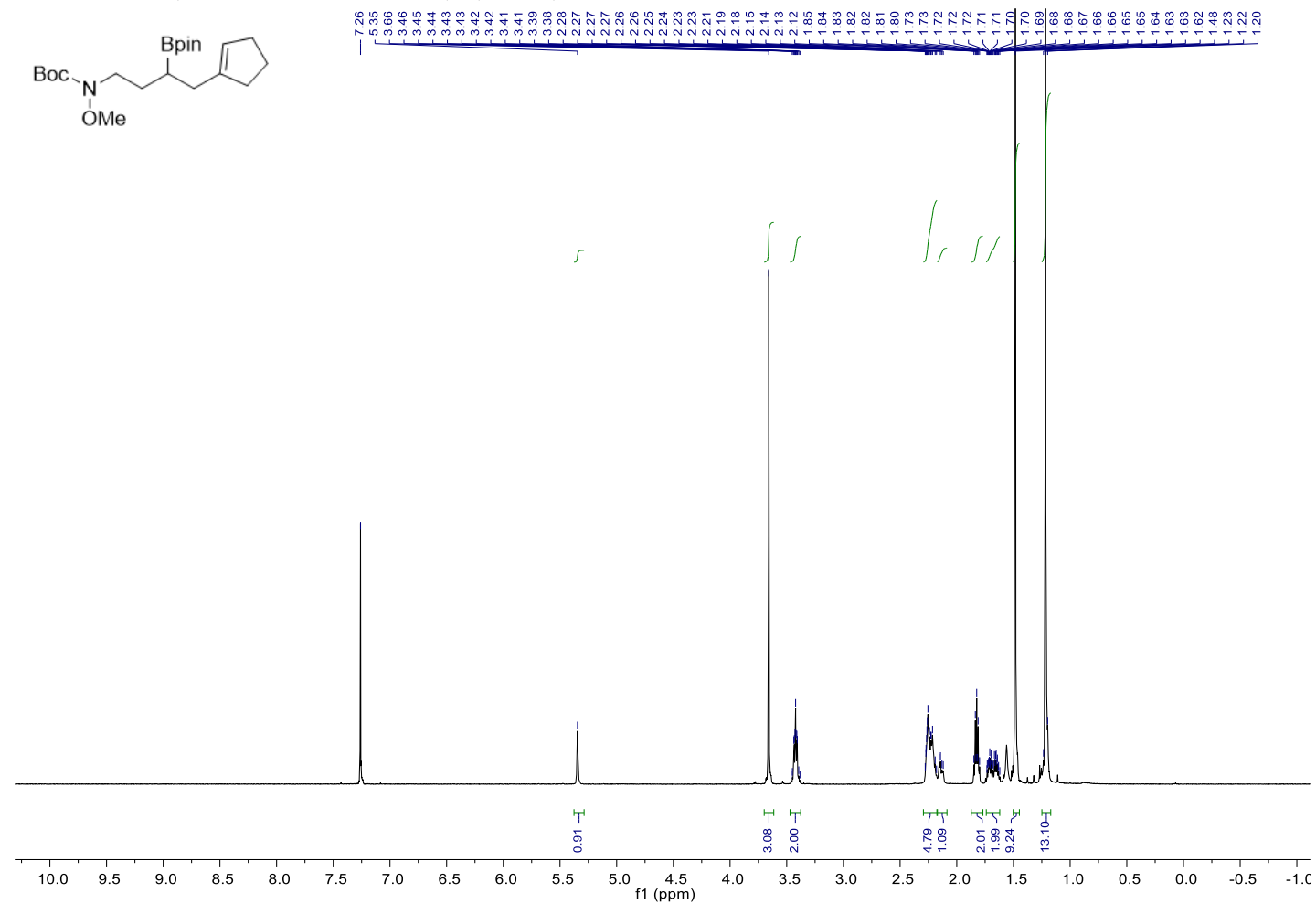

${ }^{13}$ C NMR (151 MHz, $\left.\mathrm{CDCl}_{3}\right)(\mathbf{S}-13)$

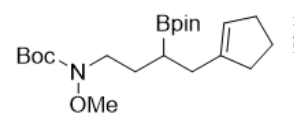

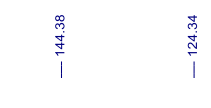
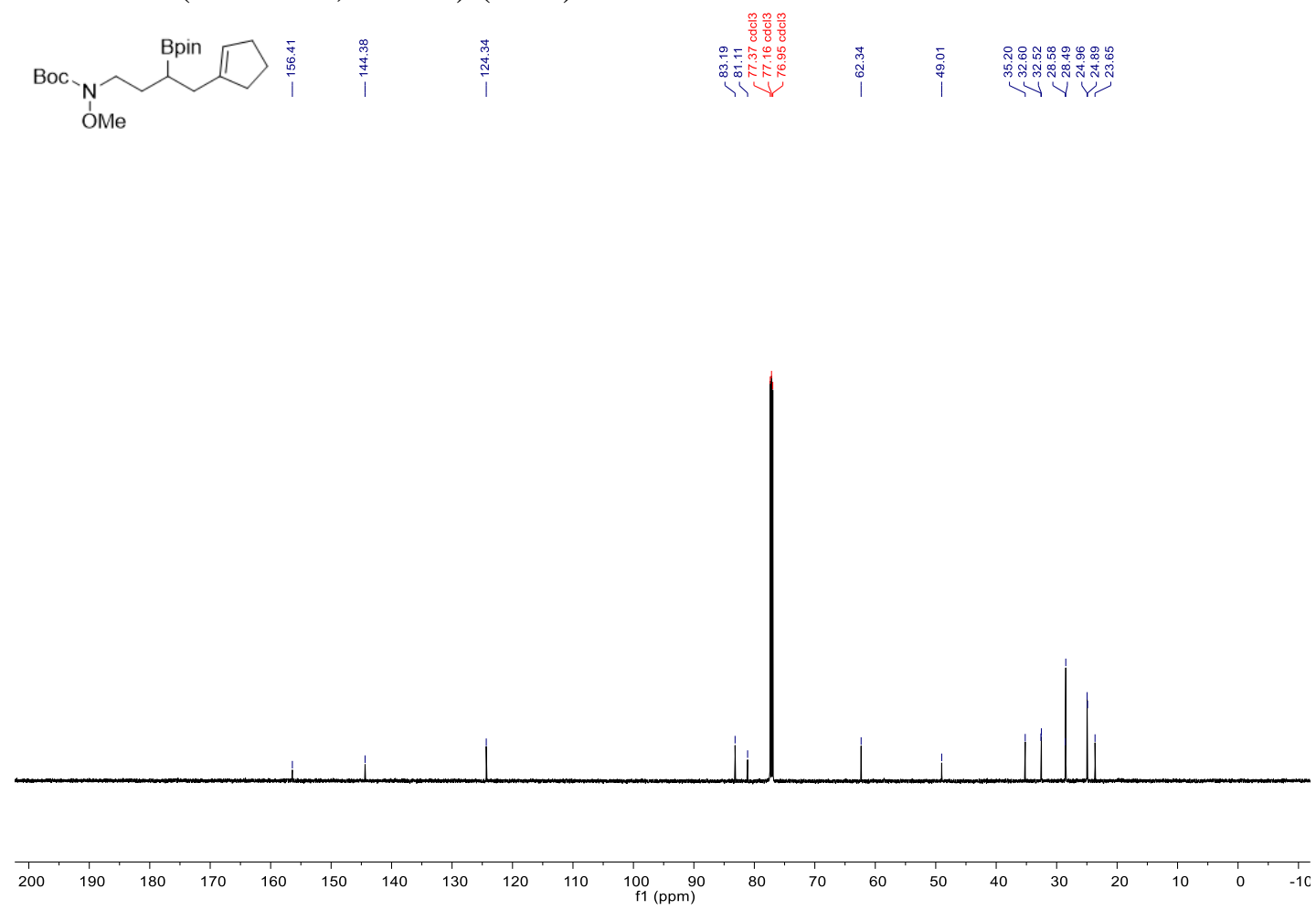


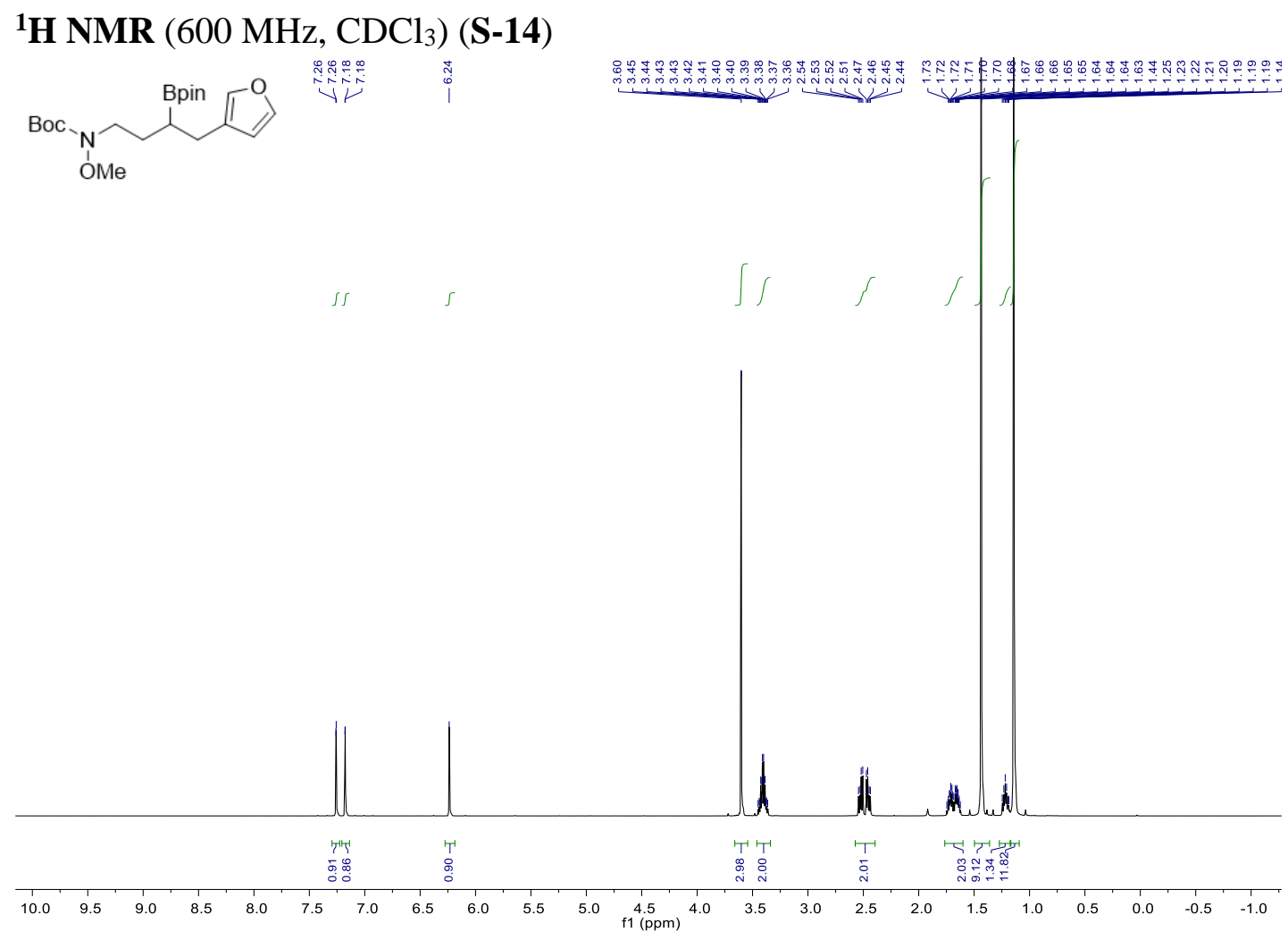

${ }^{13} \mathbf{C}$ NMR (151 MHz, $\left.\mathrm{CDCl}_{3}\right)(\mathbf{S}-14)$
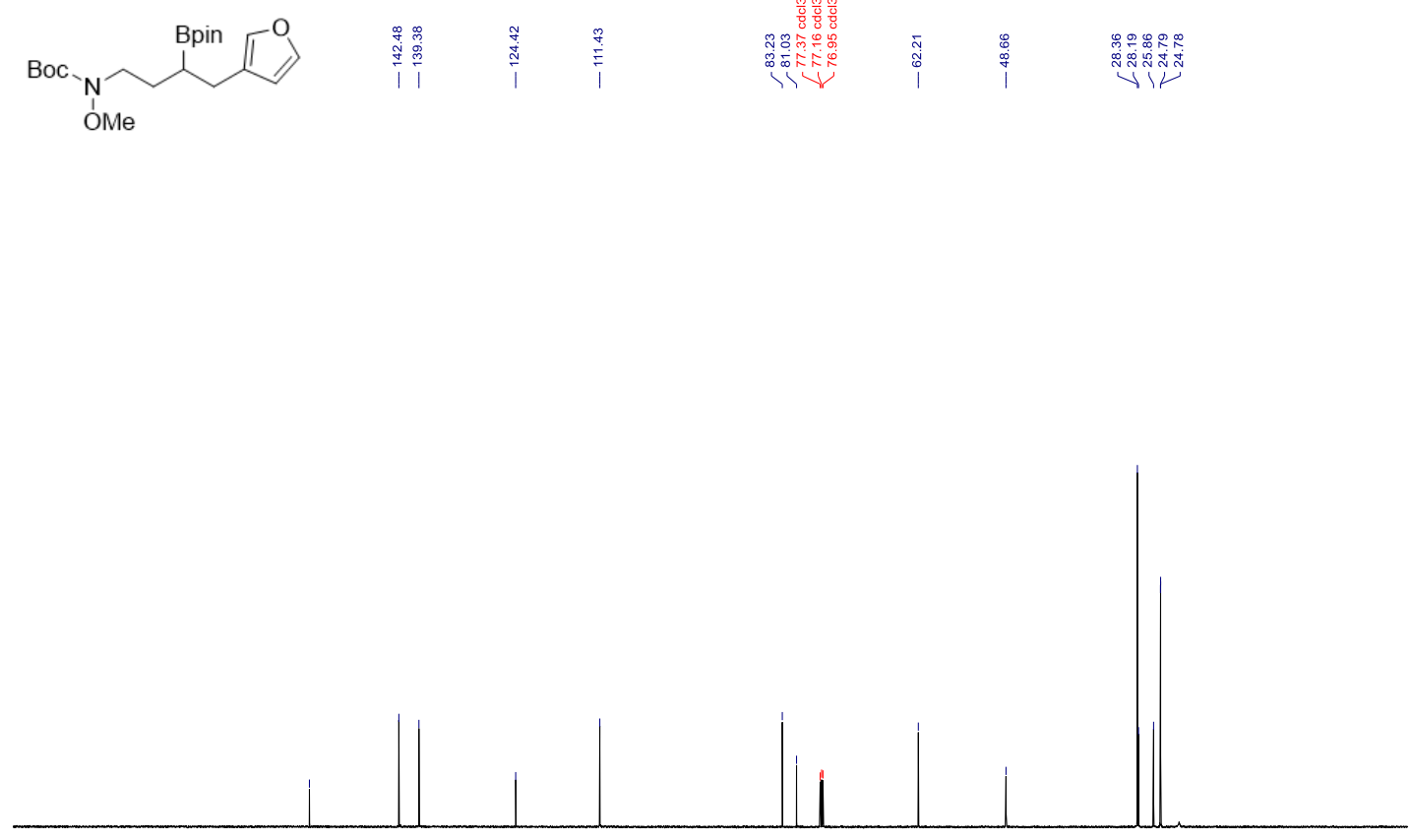

200

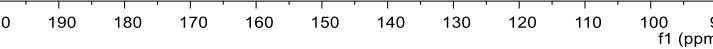




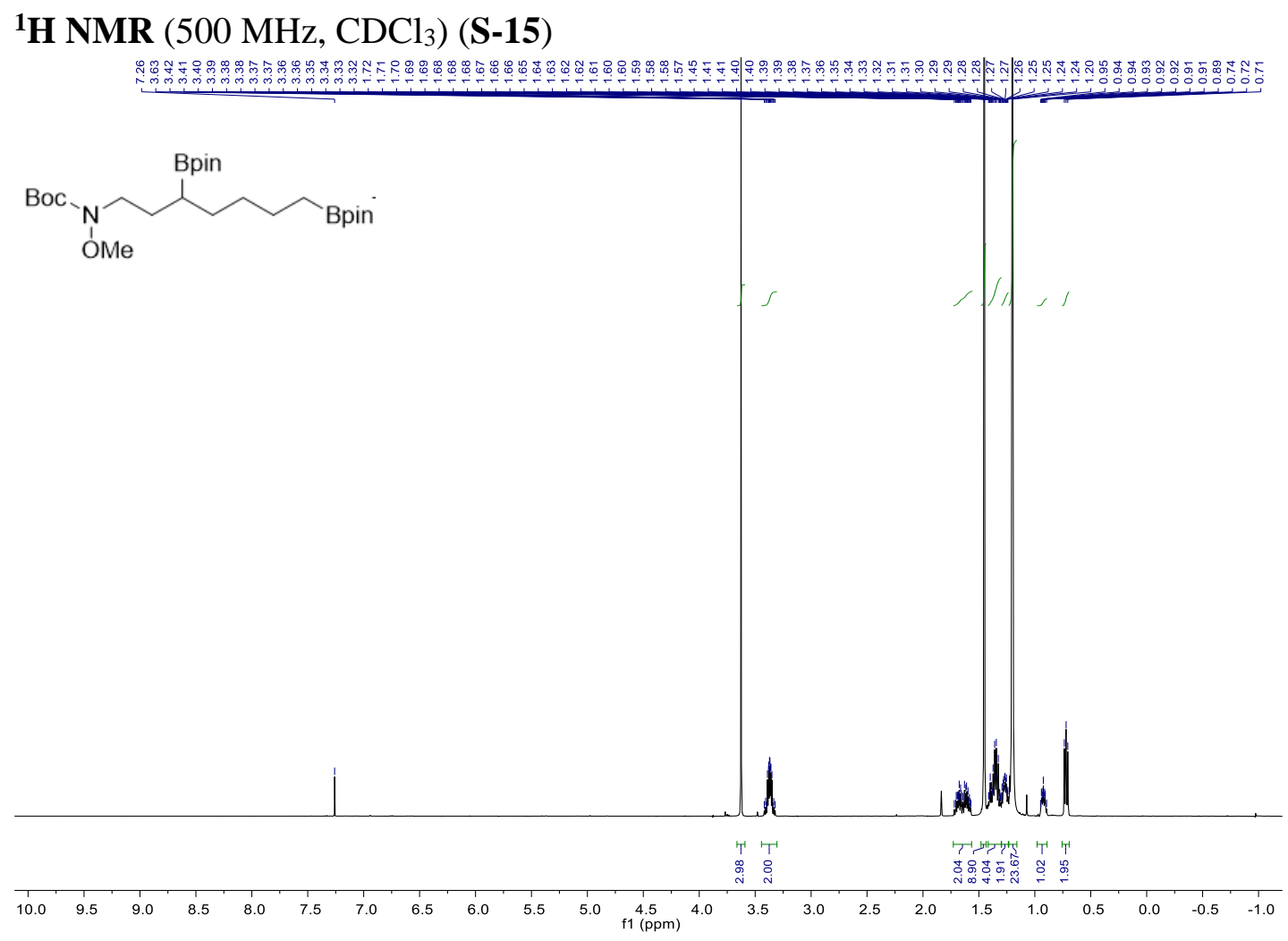

$\left.{ }^{13} \mathbf{C ~ N M R ~ ( 1 2 6 ~ M H z , ~} \mathrm{CDCl}_{3}\right)(\mathbf{S - 1 5})$
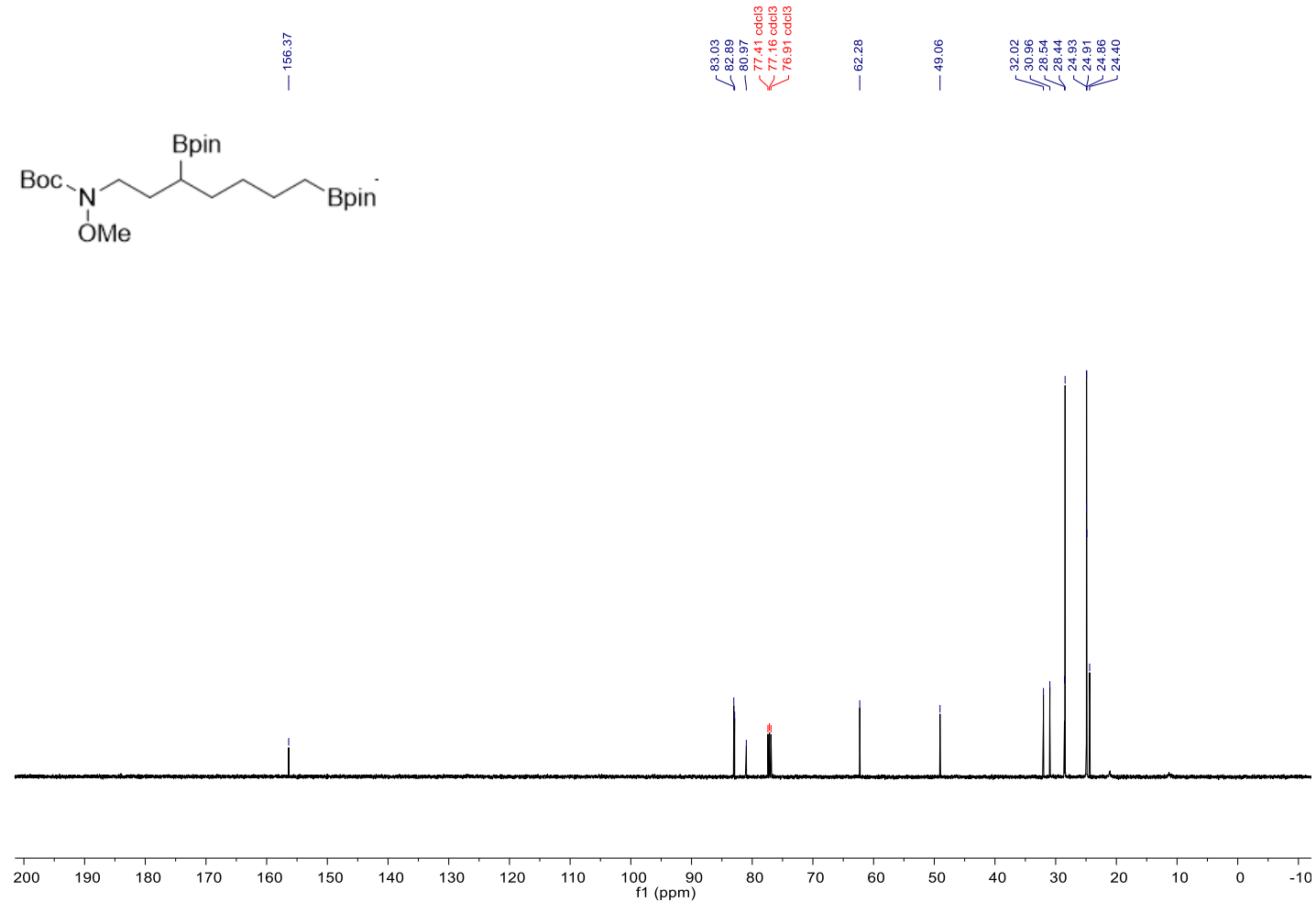


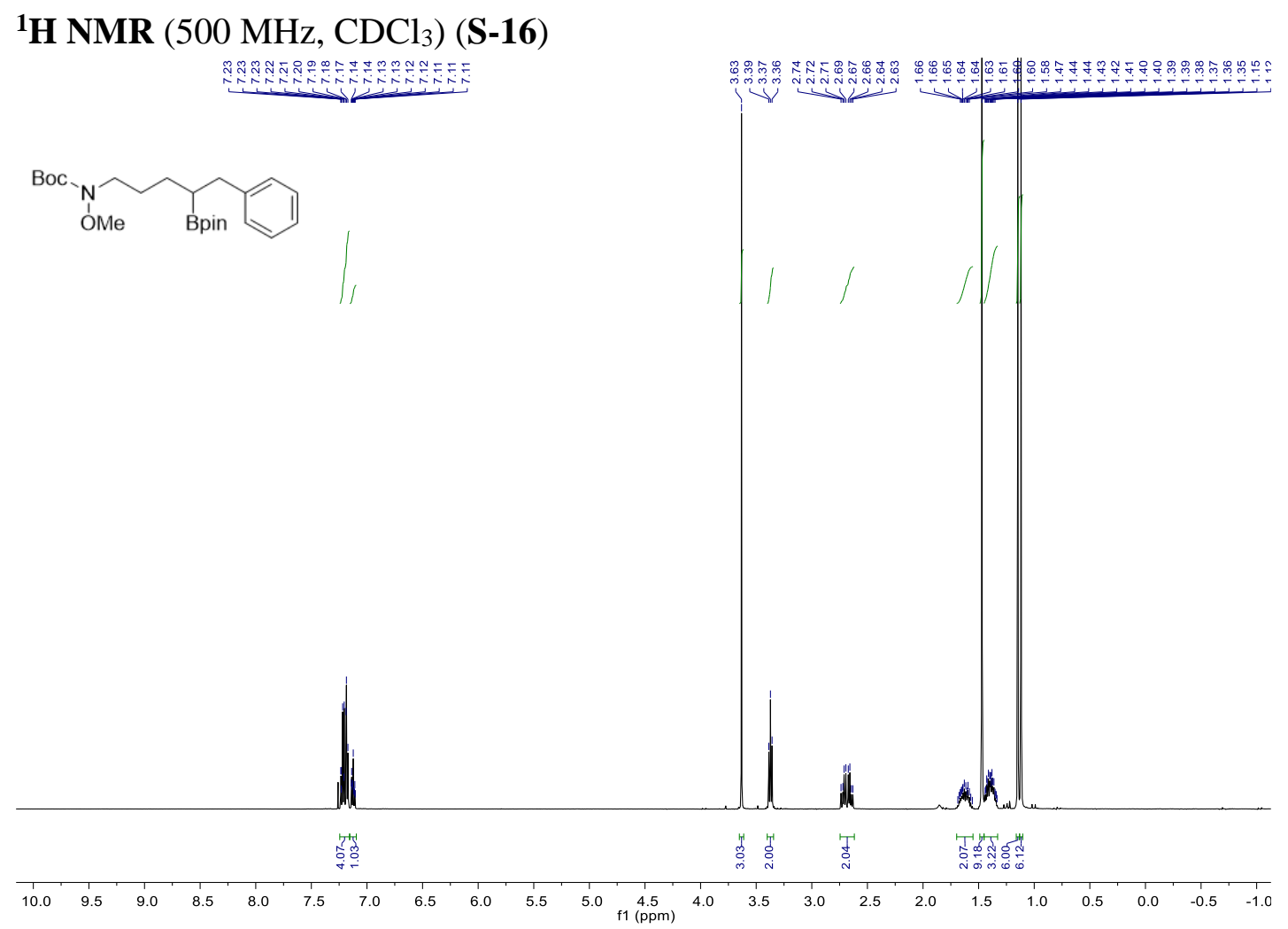

${ }^{13} \mathbf{C ~ N M R}\left(126 \mathrm{MHz}, \mathrm{CDCl}_{3}\right)(\mathbf{S - 1 6})$
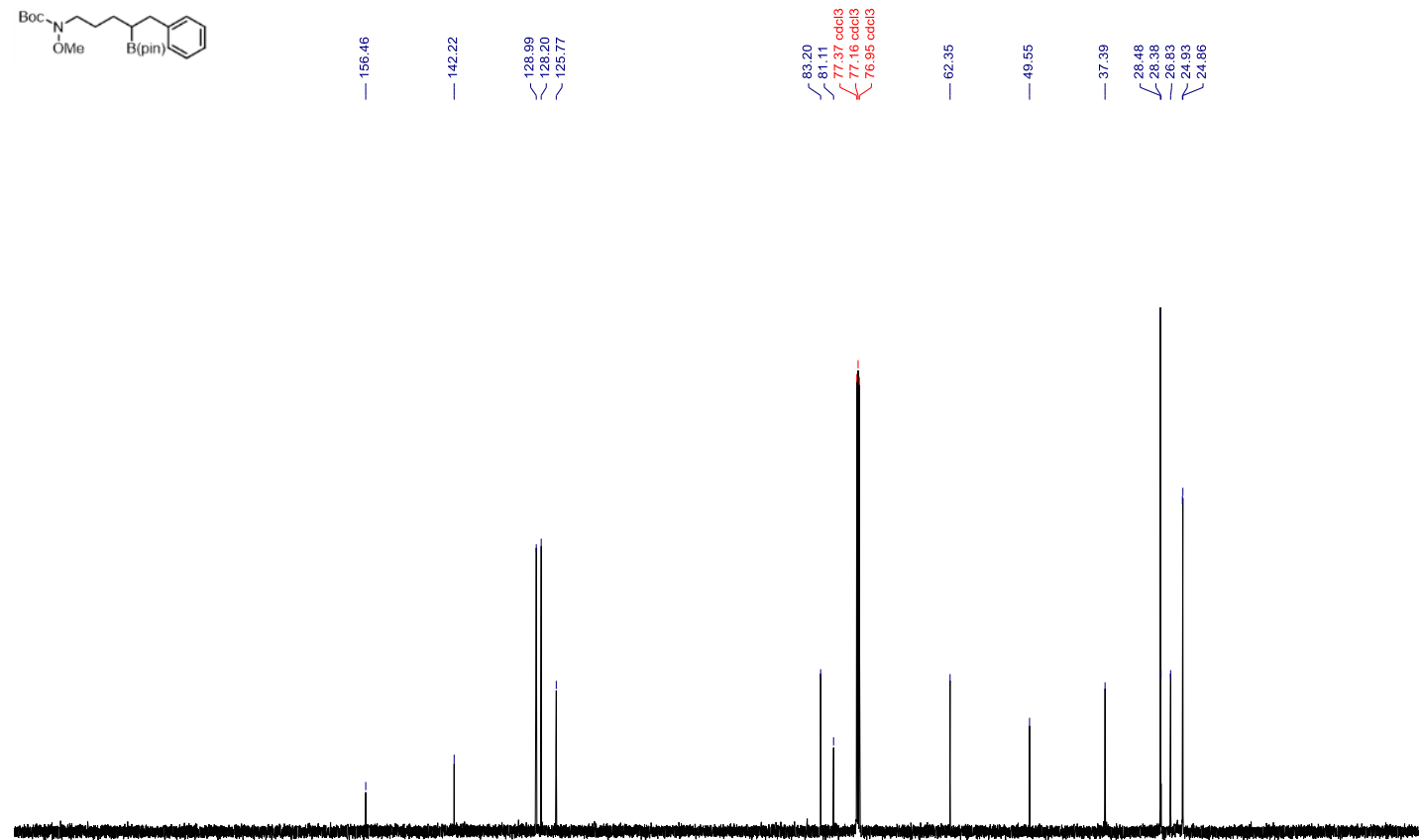

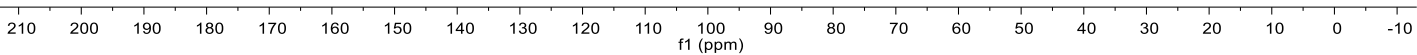




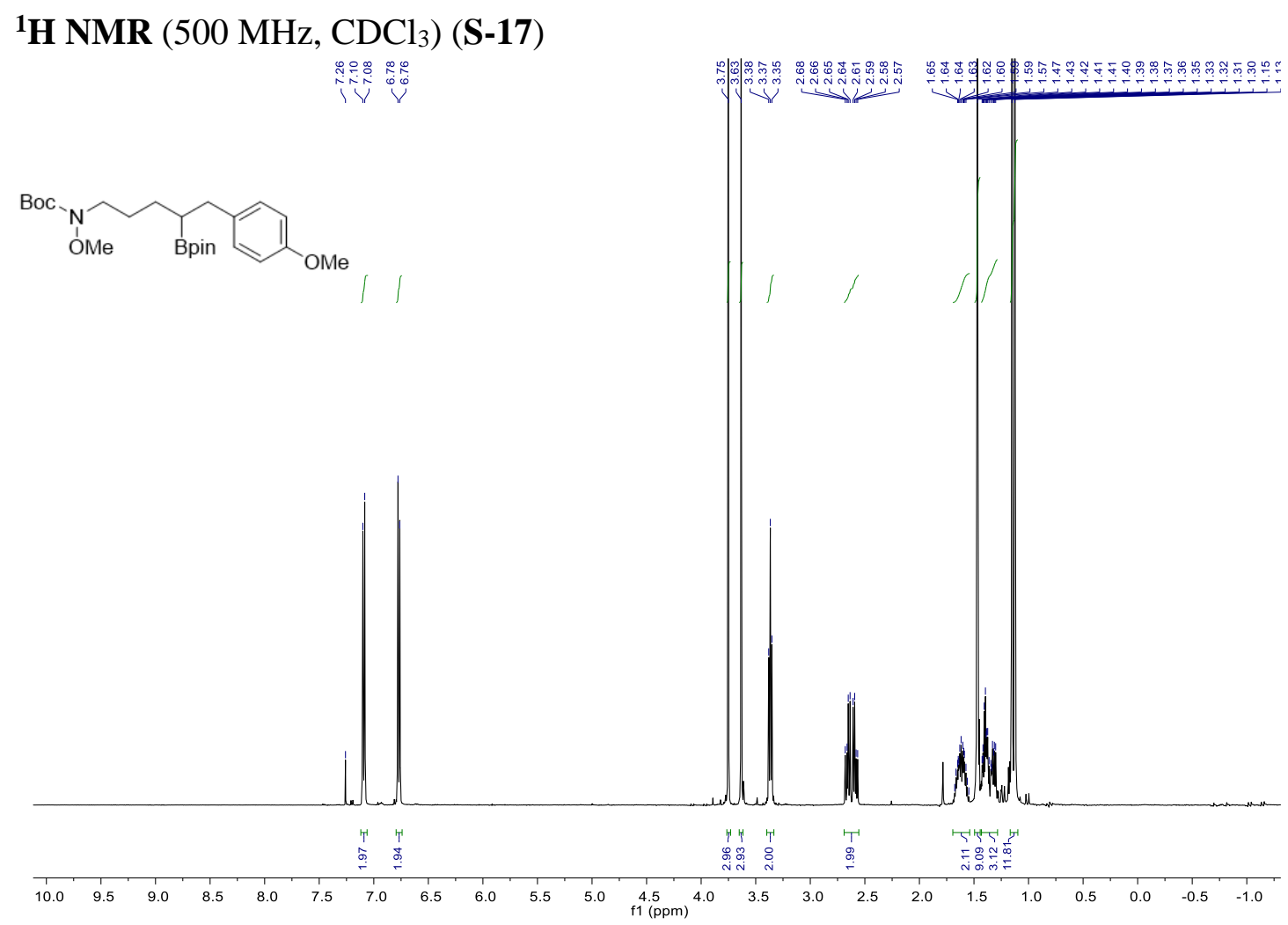

${ }^{13} \mathbf{C ~ N M R}\left(126 \mathrm{MHz}, \mathrm{CDCl}_{3}\right)$ (S-17)
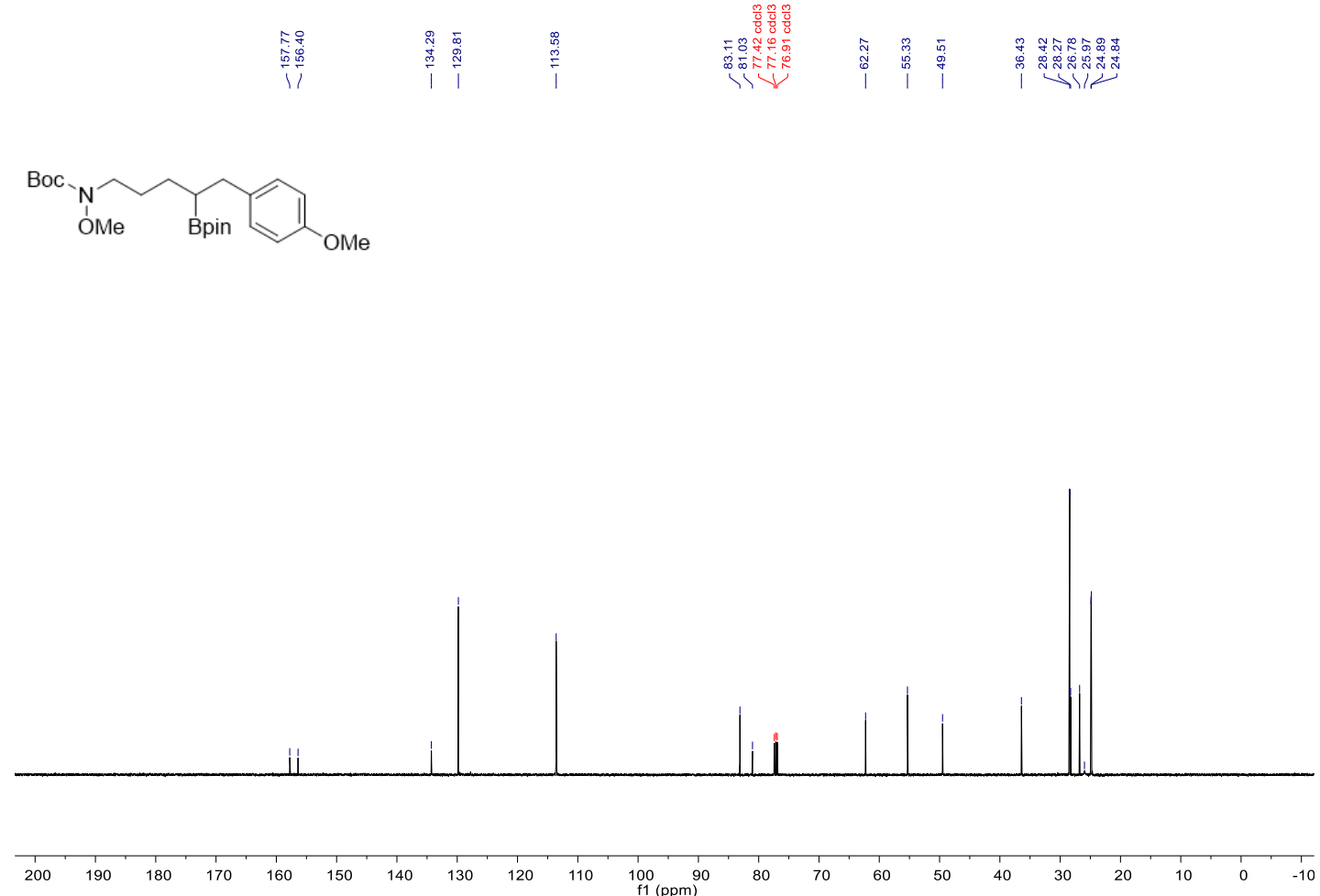


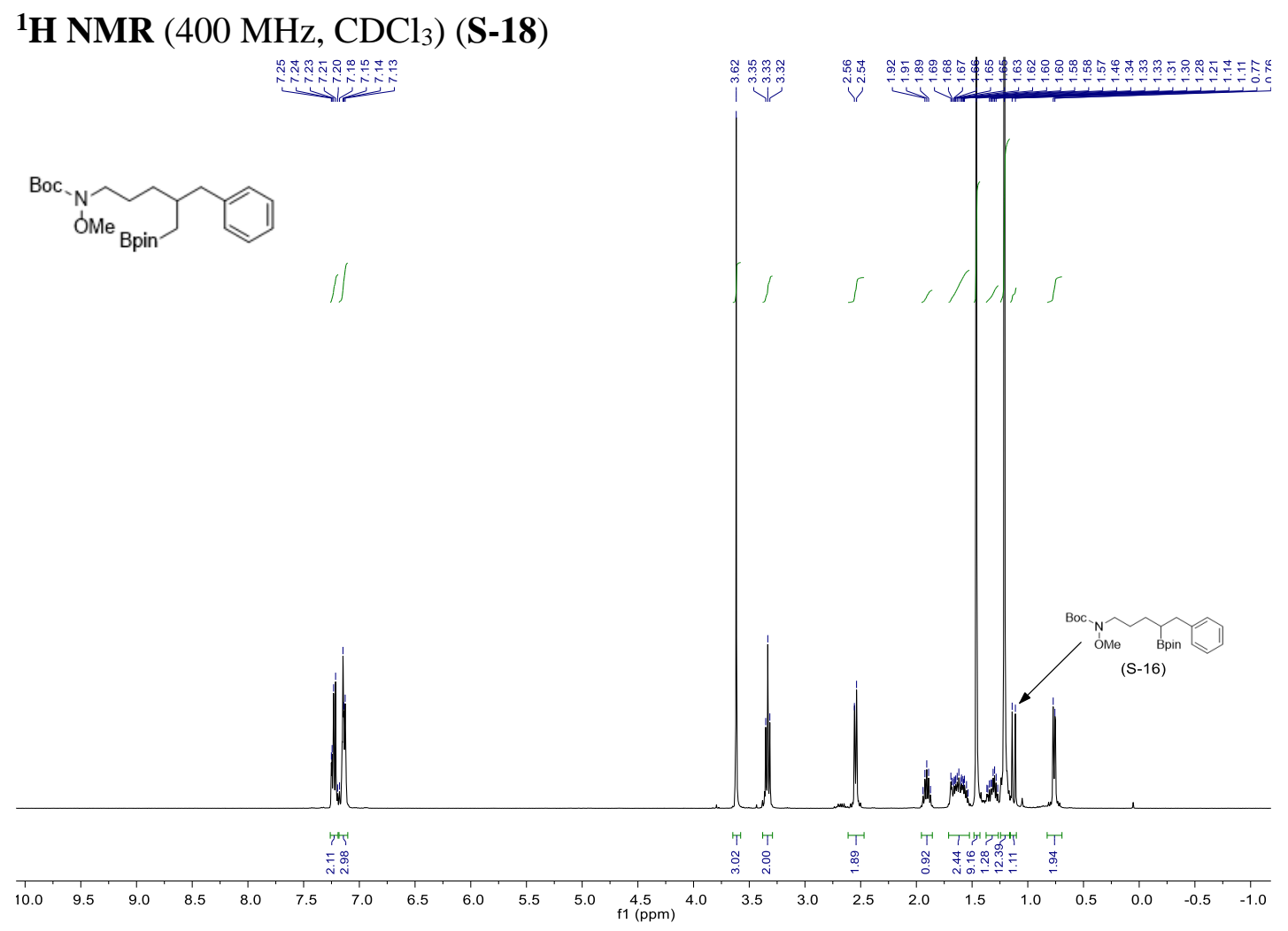

${ }^{13} \mathbf{C ~ N M R}\left(151 \mathrm{MHz}, \mathrm{CDCl}_{3}\right)(\mathbf{S}-\mathbf{1 8})$
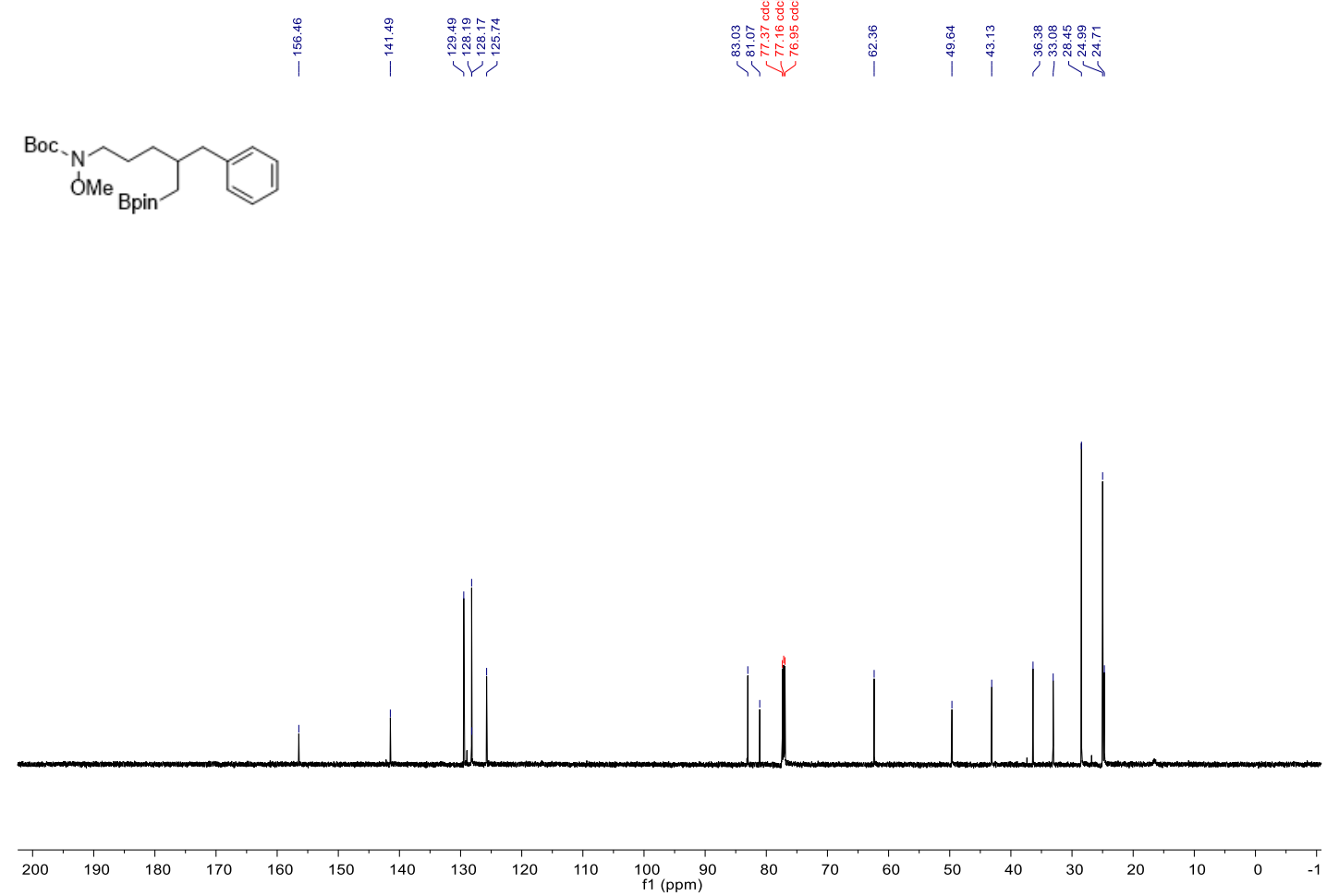
${ }^{1} \mathbf{H}$ NMR (600 MHz, $\left.\mathrm{CDCl}_{3}\right)(\mathbf{S - 1 9})$

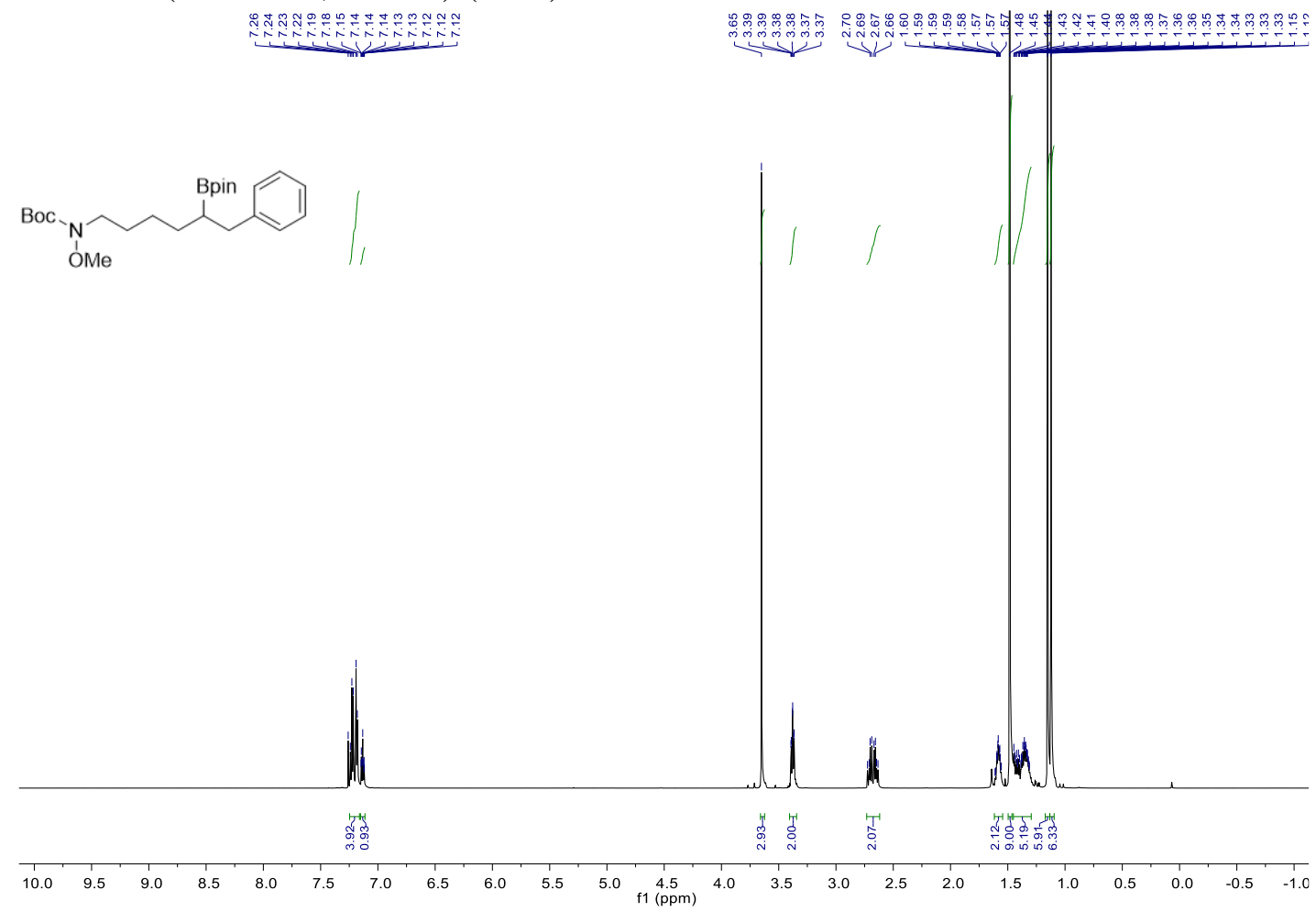

${ }^{13}$ C NMR (151 MHz, $\left.\mathrm{CDCl}_{3}\right)$ (S-19)
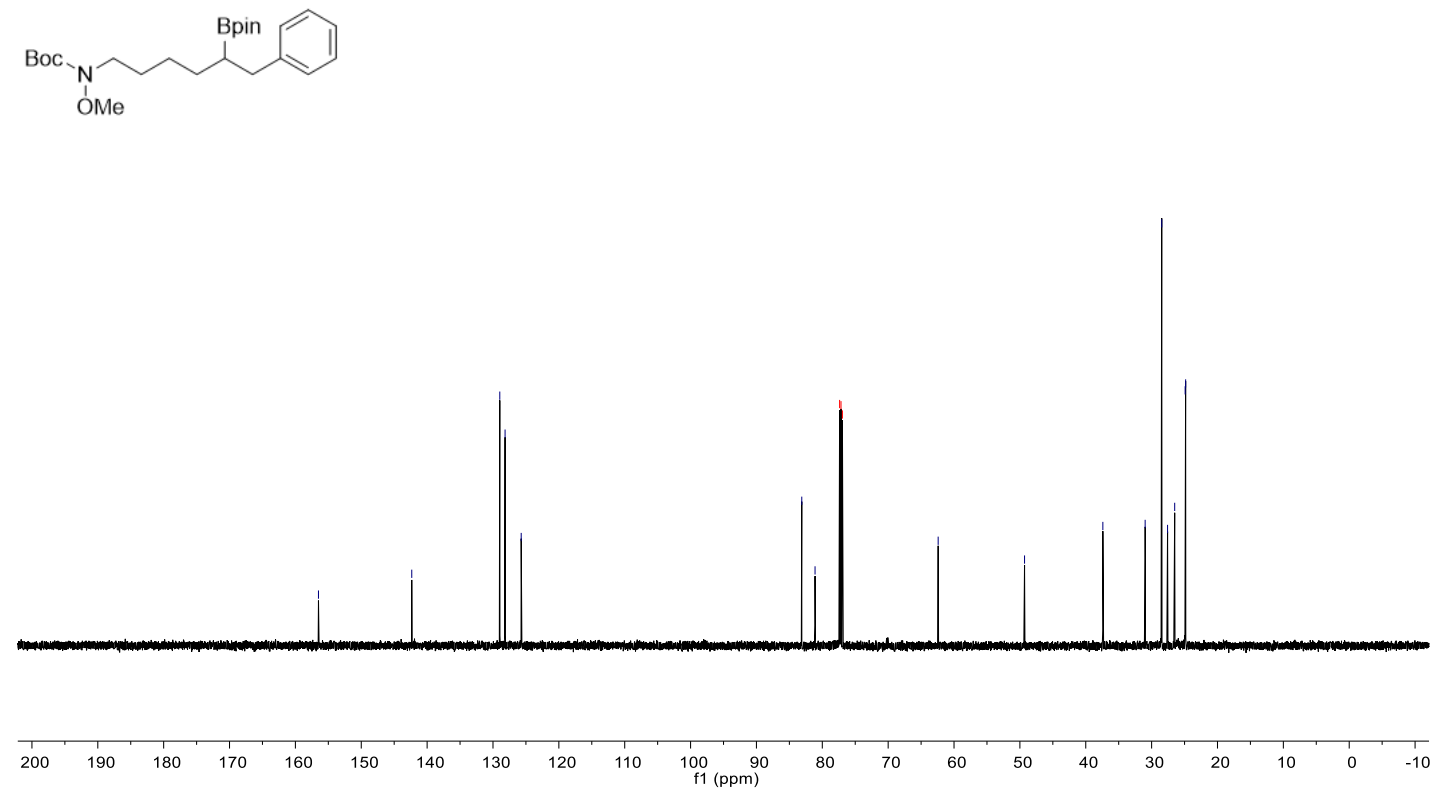


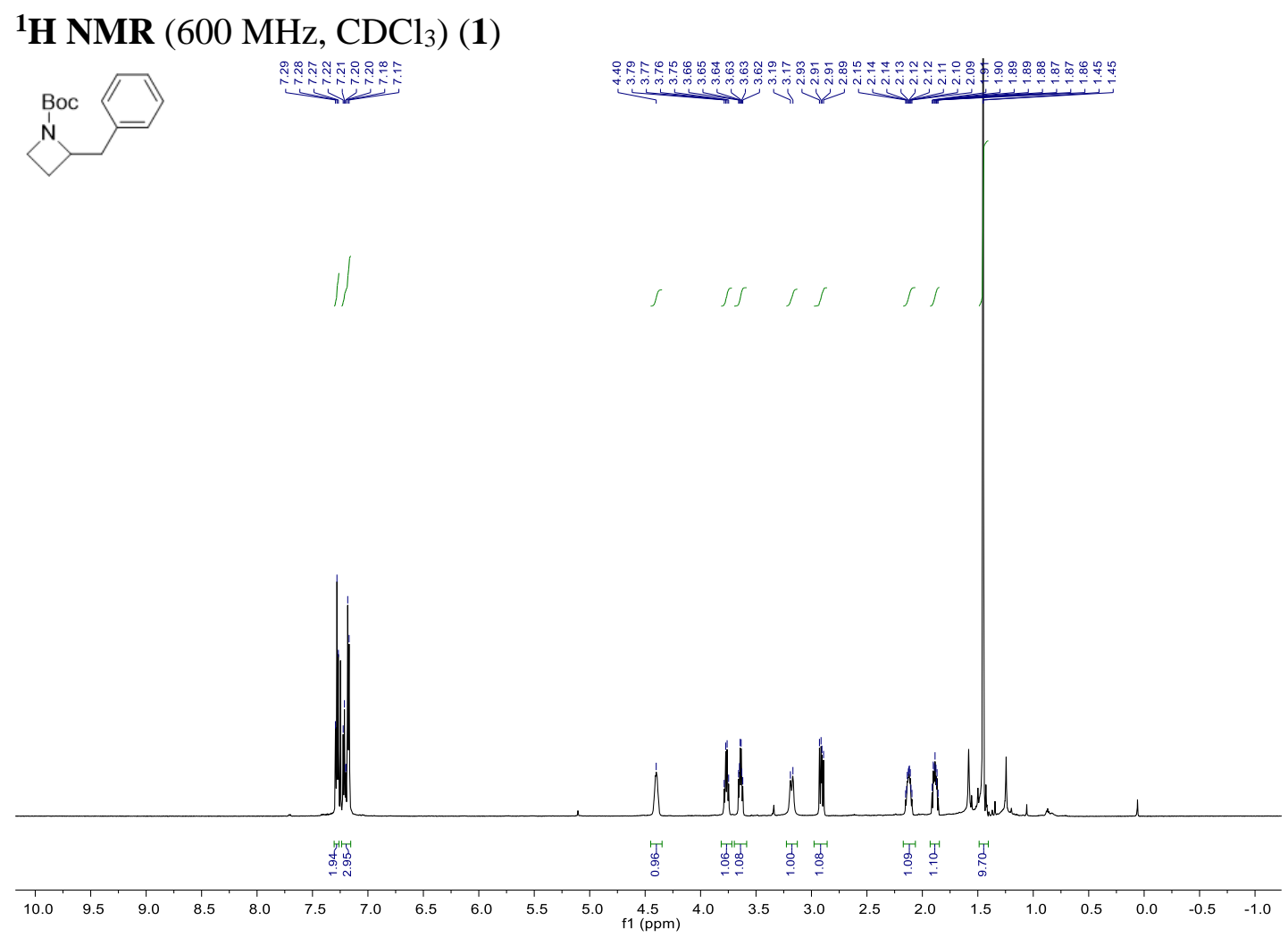

${ }^{13}$ C NMR (151 MHz, $\left.\mathrm{CDCl}_{3}\right)$ (1)
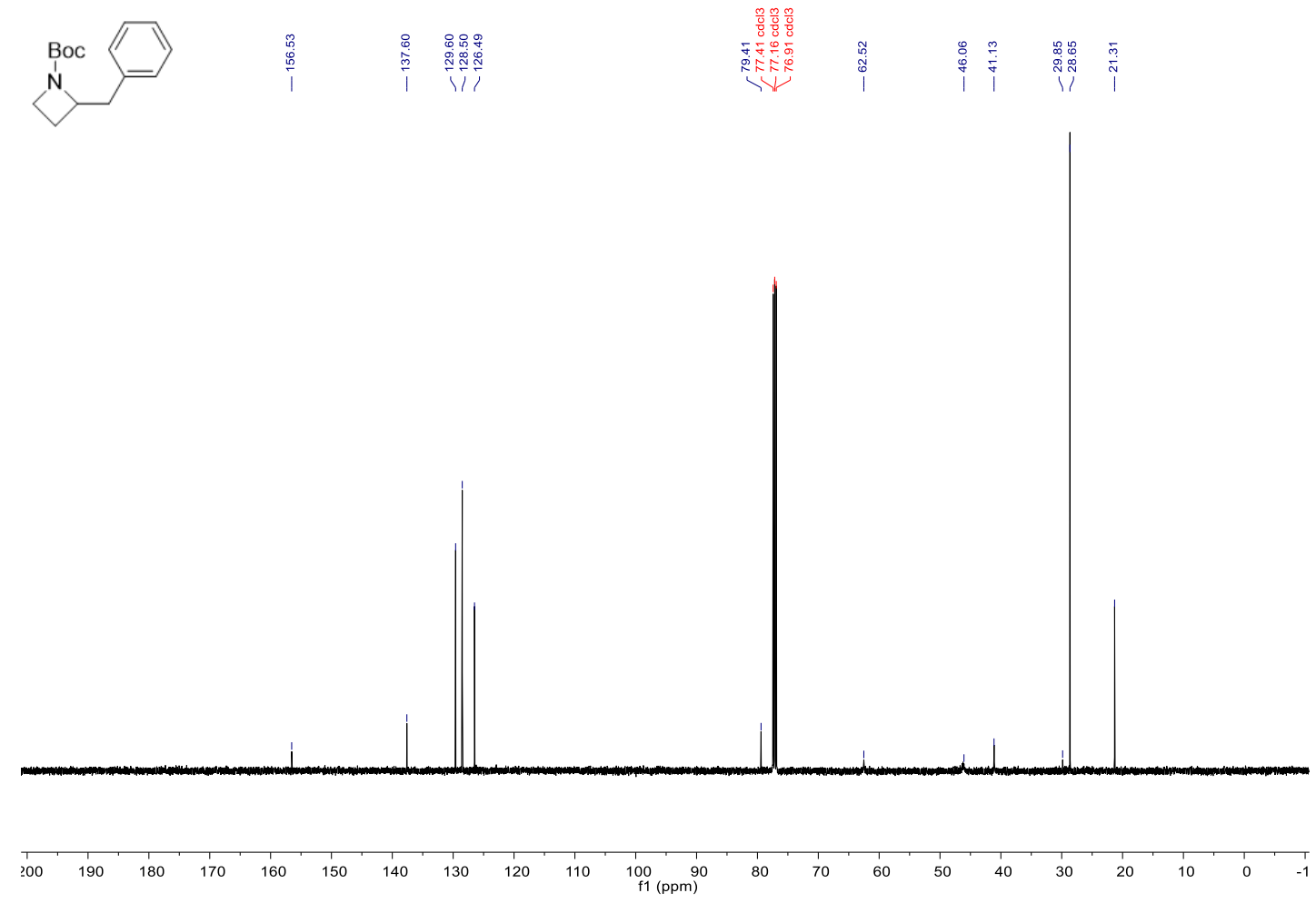


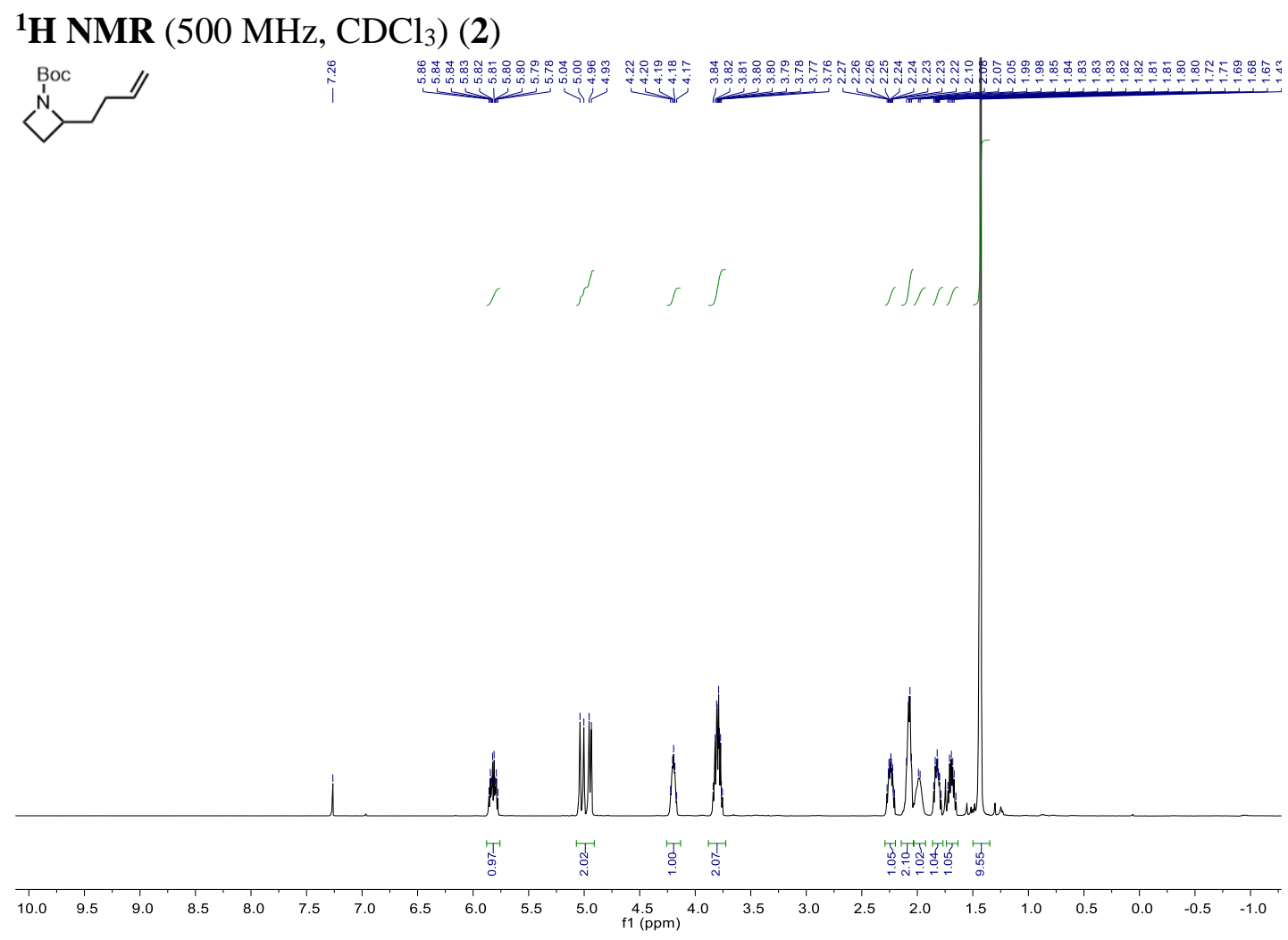

${ }^{13} \mathbf{C ~ N M R}\left(126 \mathrm{MHz}, \mathrm{CDCl}_{3}\right)$ (2)
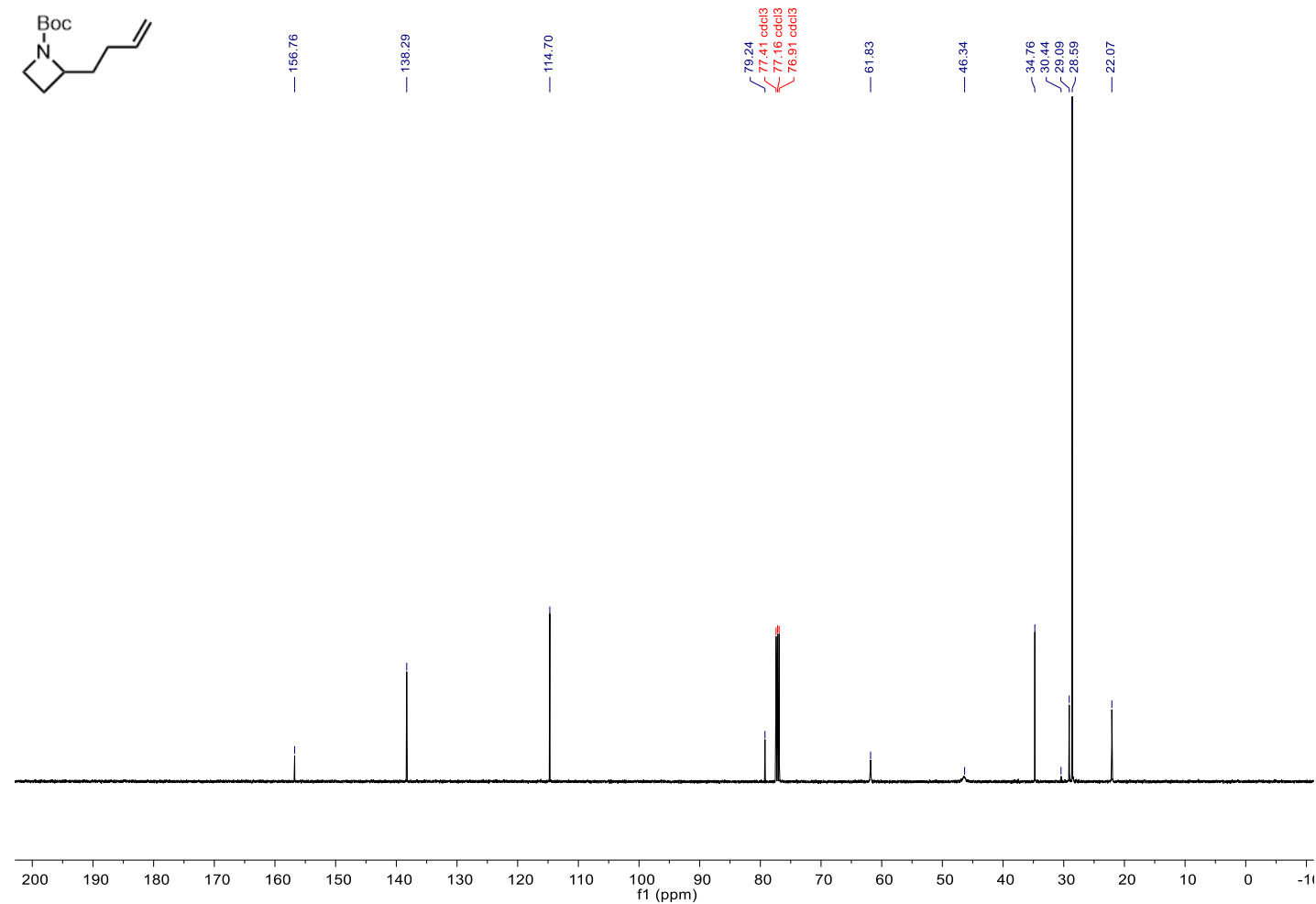


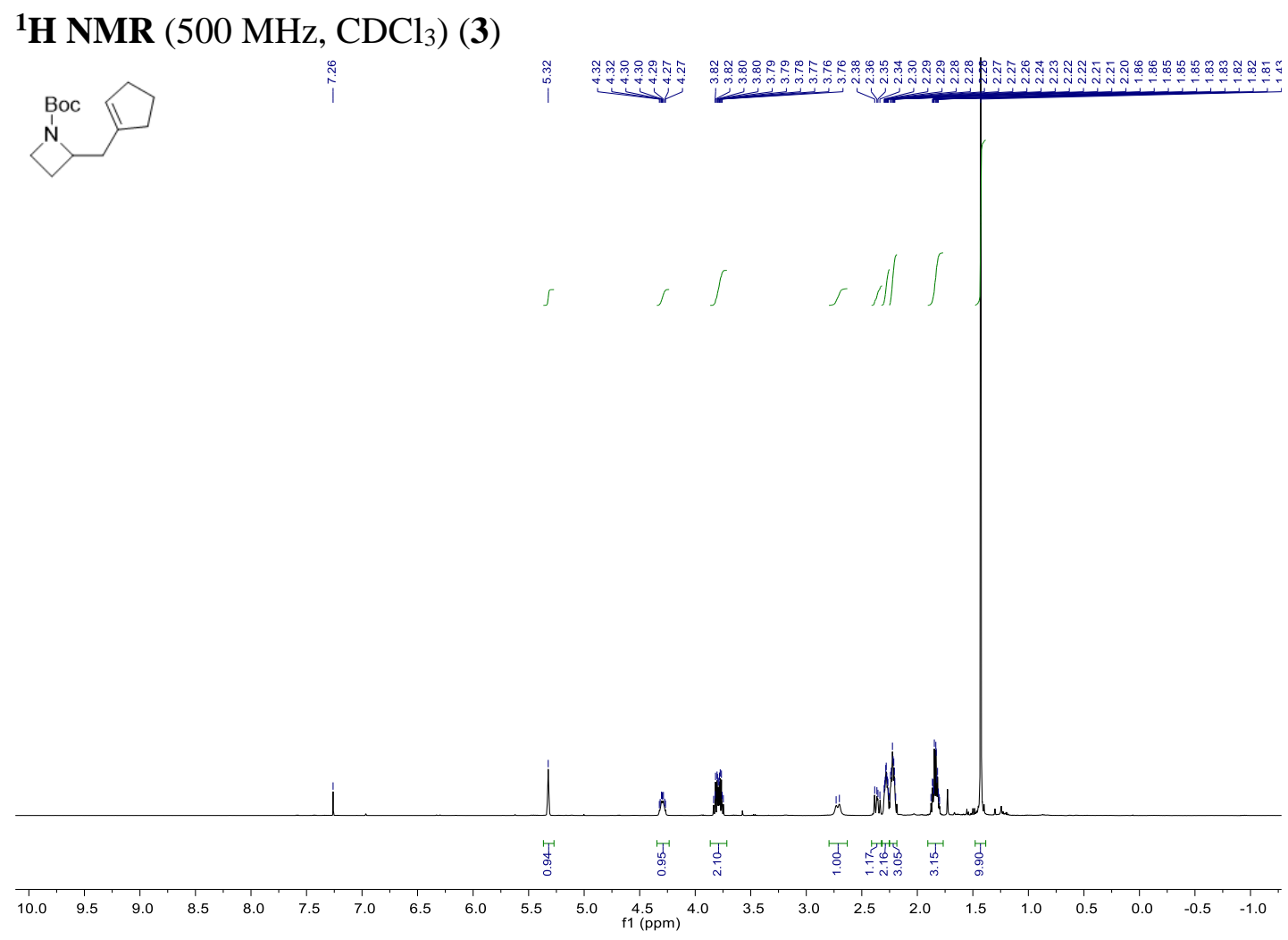

${ }^{13} \mathrm{C}$ NMR (126 MHz, $\left.\mathrm{CDCl}_{3}\right)$ (3)
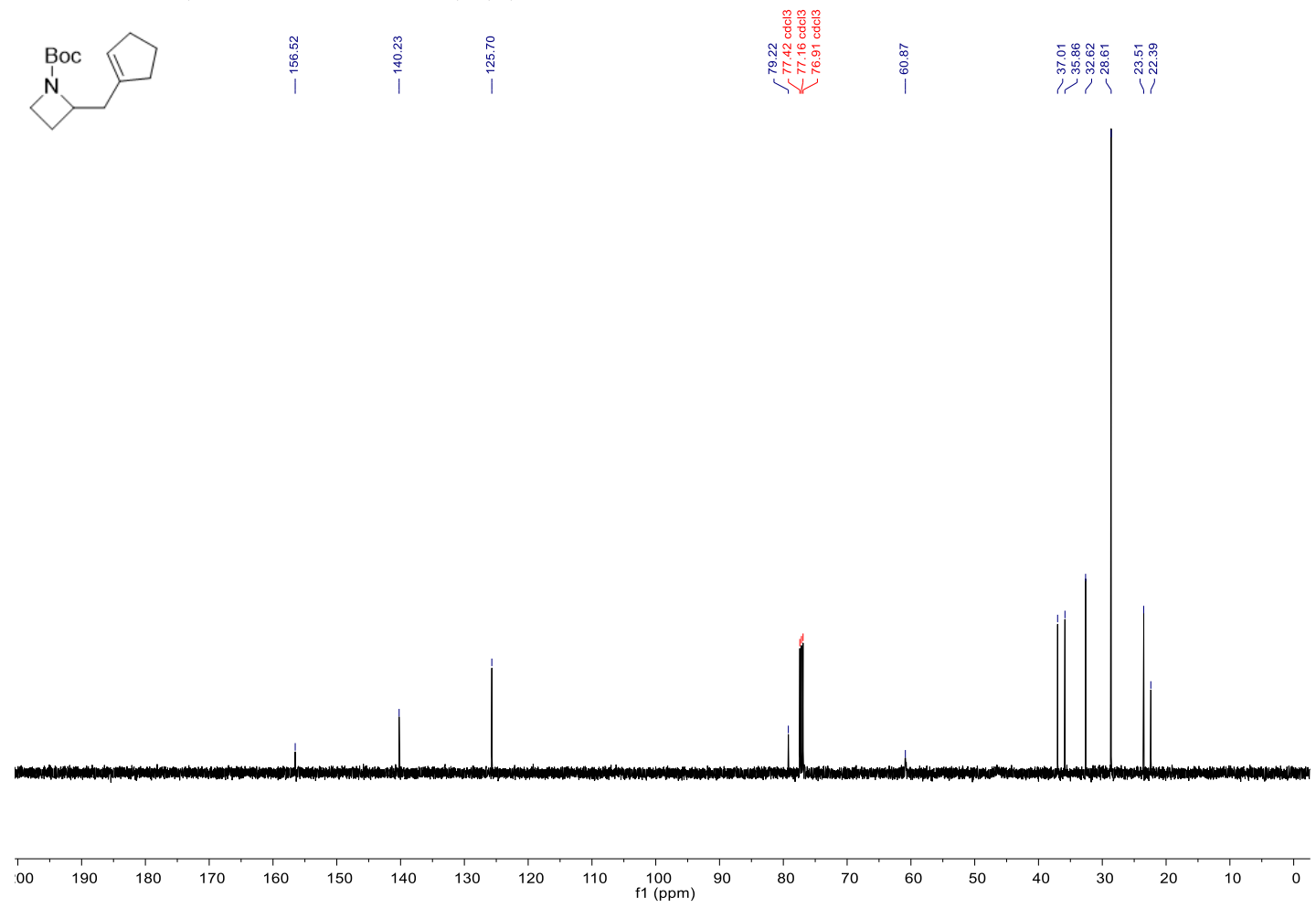
Xu, Zhang, Ingoglia, Allais, Dechert-Schmitt, Singer and Morken Supporting Information

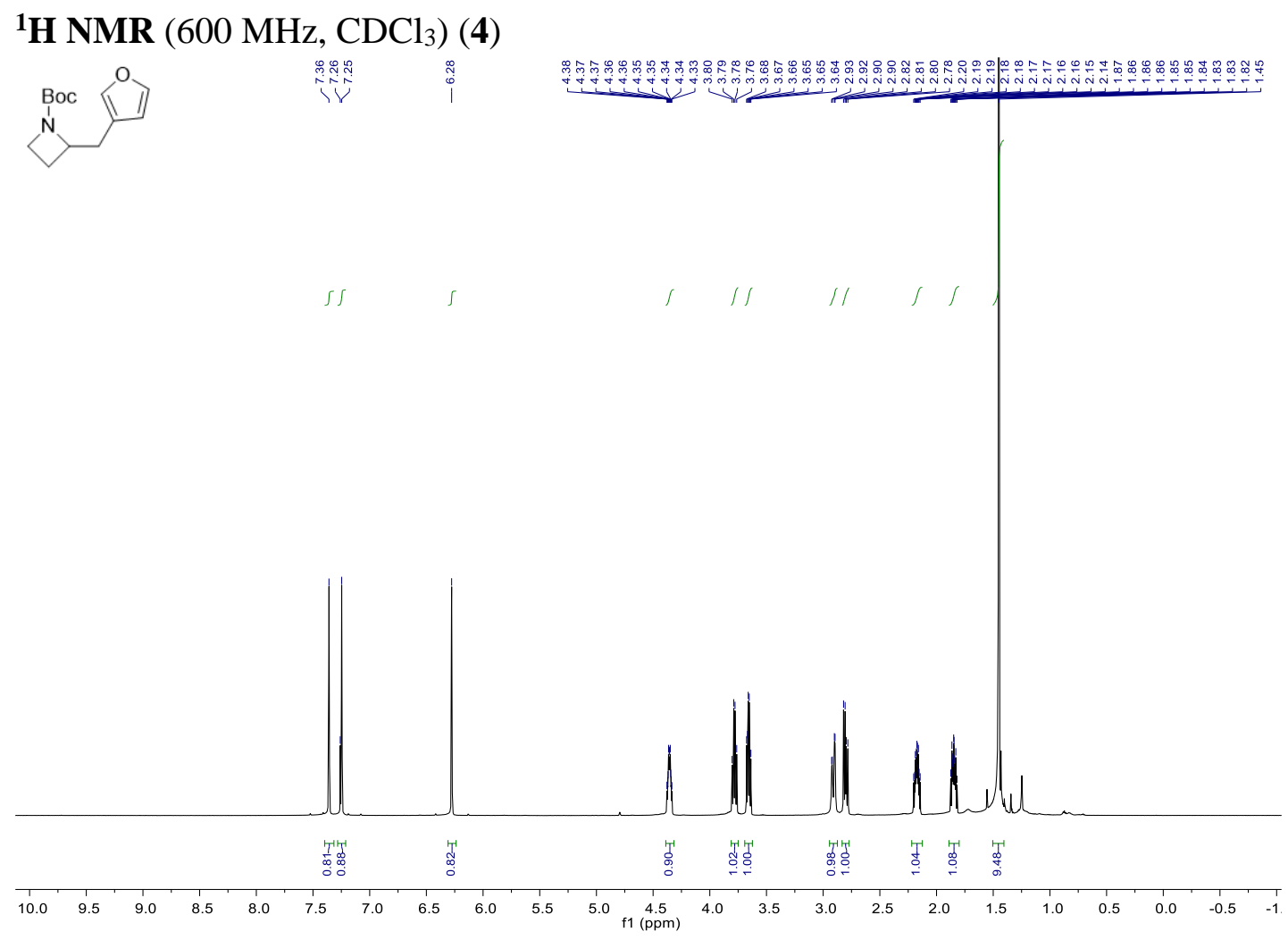

${ }^{13} \mathrm{C}$ NMR (151 MHz, $\mathrm{CDCl}_{3}$ ) (4)
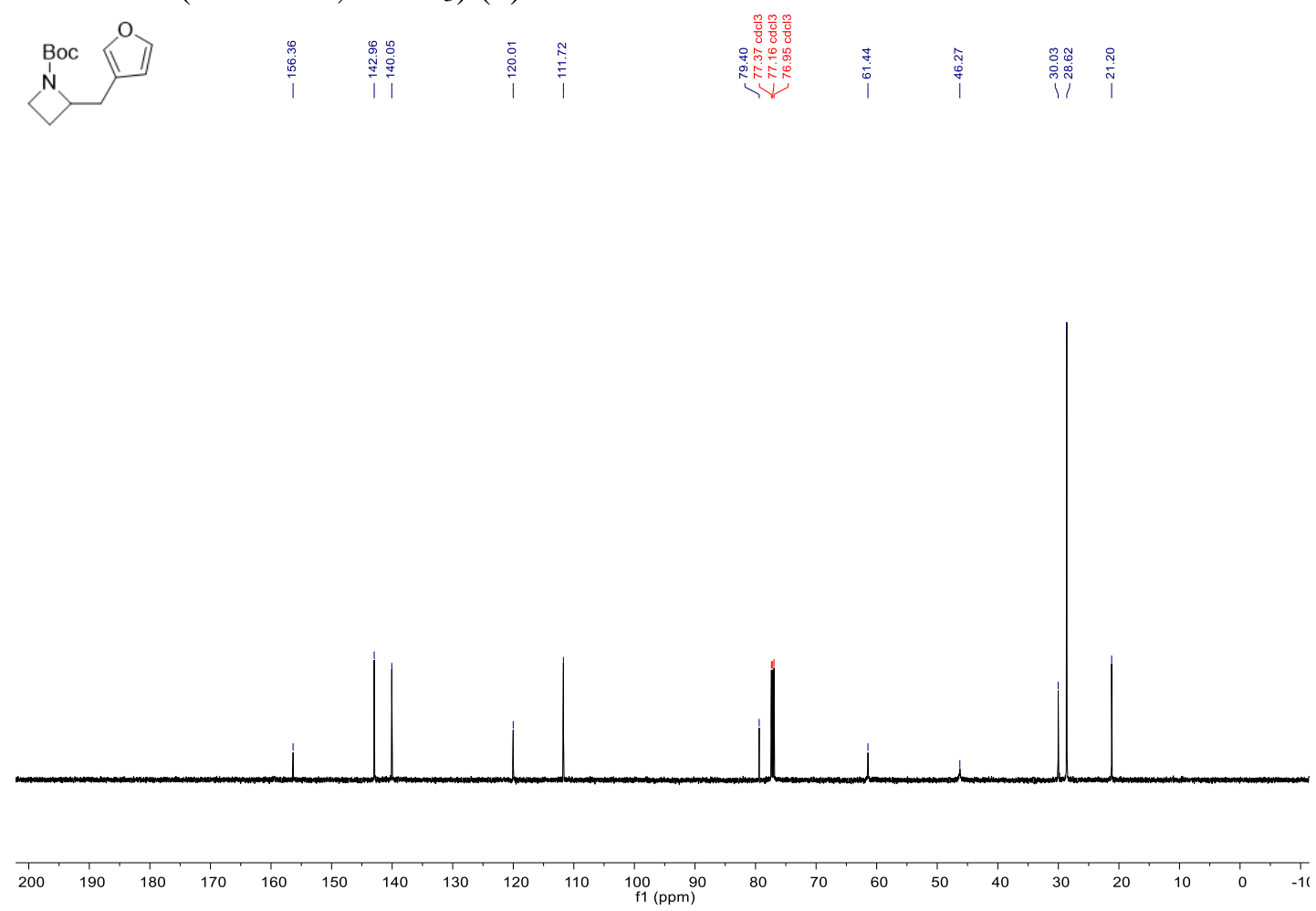
Xu, Zhang, Ingoglia, Allais, Dechert-Schmitt, Singer and Morken Supporting Information

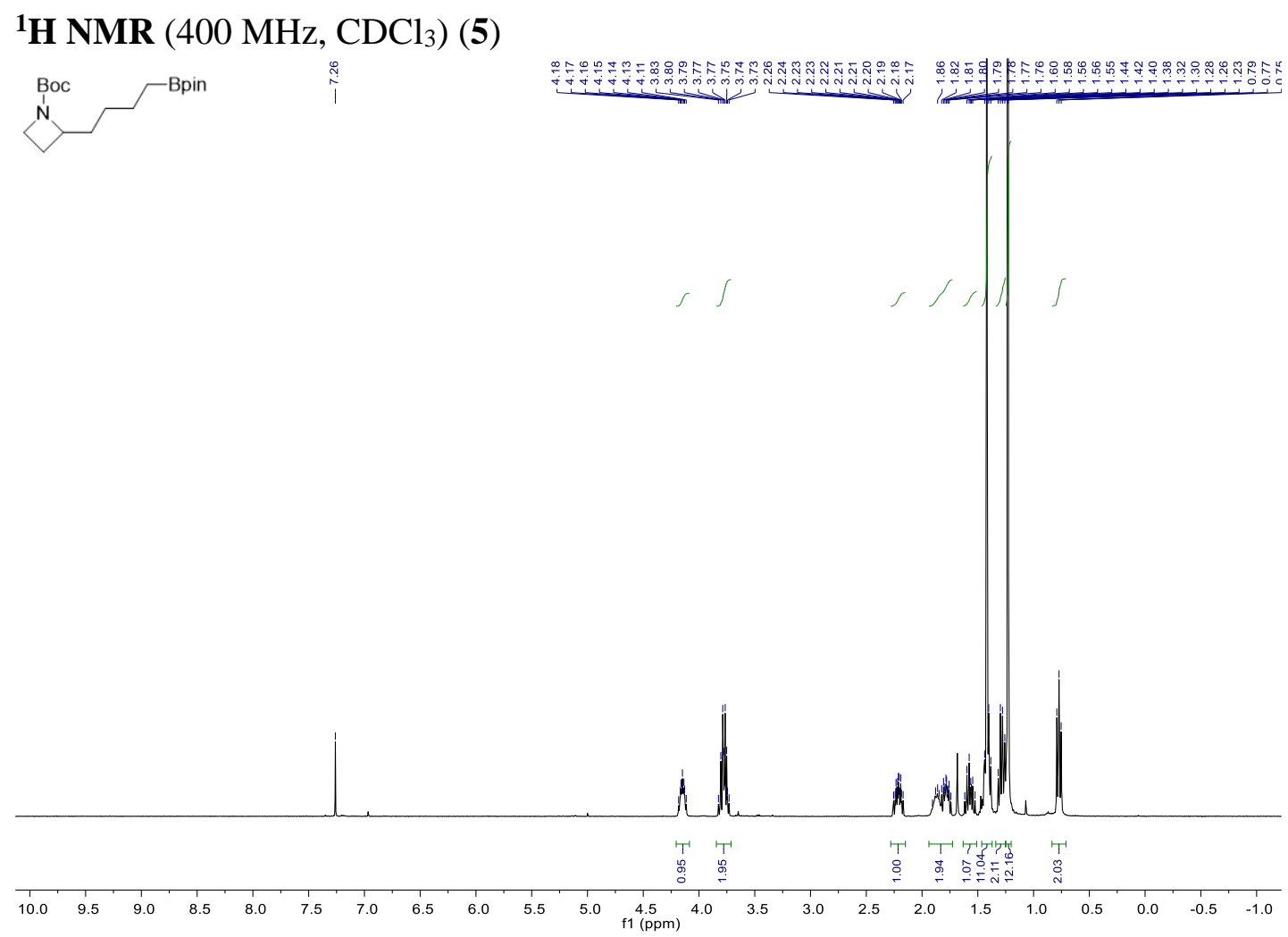

${ }^{13} \mathrm{C}$ NMR (151 MHz, $\mathrm{CDCl}_{3}$ ) (5)
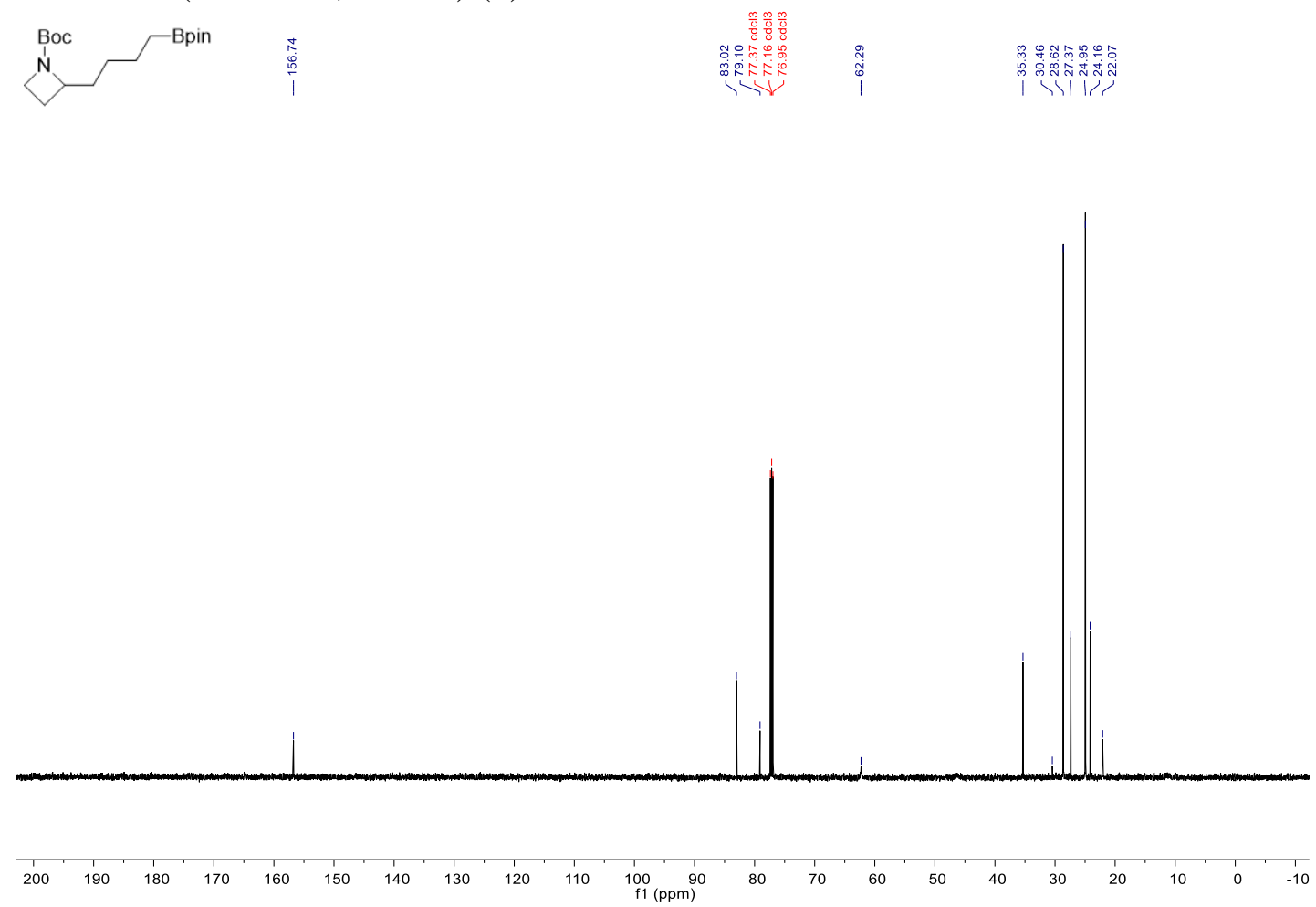
Xu, Zhang, Ingoglia, Allais, Dechert-Schmitt, Singer and Morken Supporting Information

\section{${ }^{1} \mathbf{H}$ NMR $\left(500 \mathrm{MHz}, \mathrm{CDCl}_{3}\right)(\mathbf{6})$}

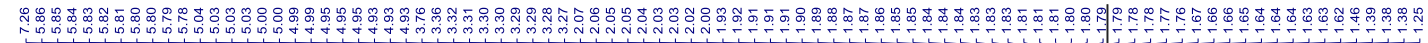

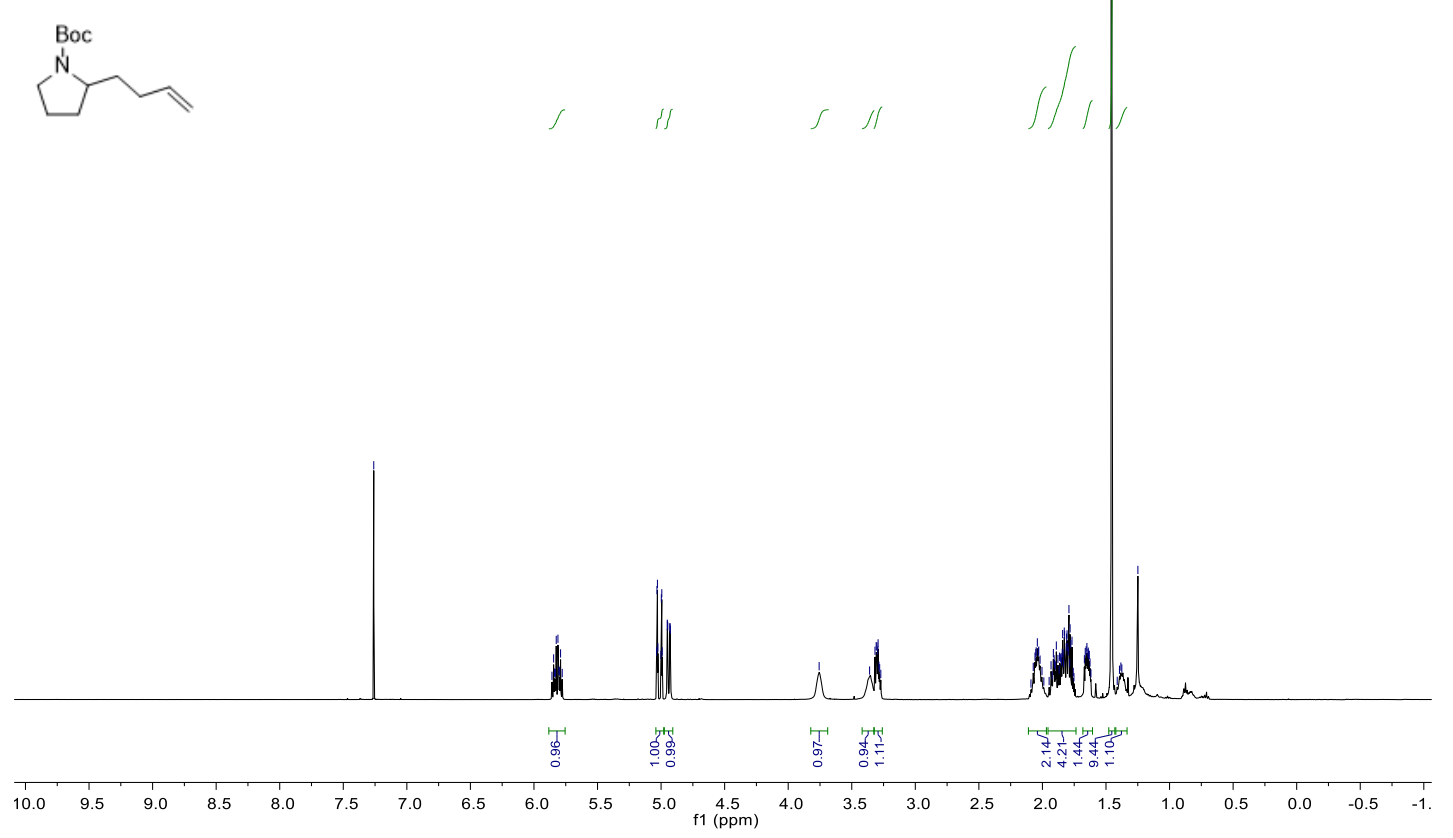

${ }^{13} \mathbf{C}$ NMR (151 MHz, $\left.\mathrm{CDCl}_{3}\right)(\mathbf{6})$

$\bigsqcup^{\text {Boc }}$

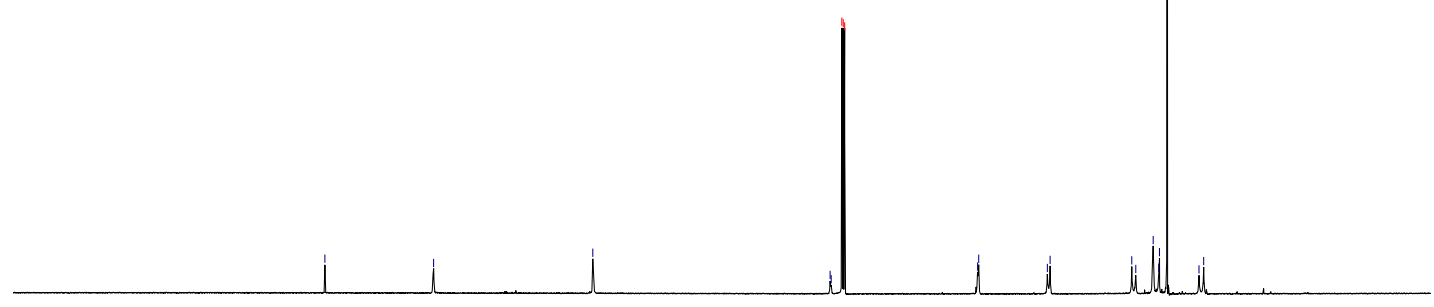

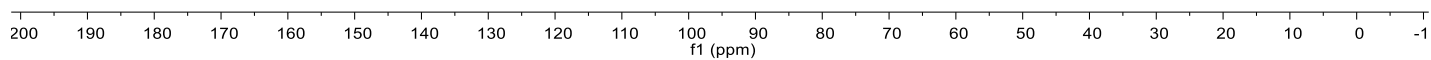




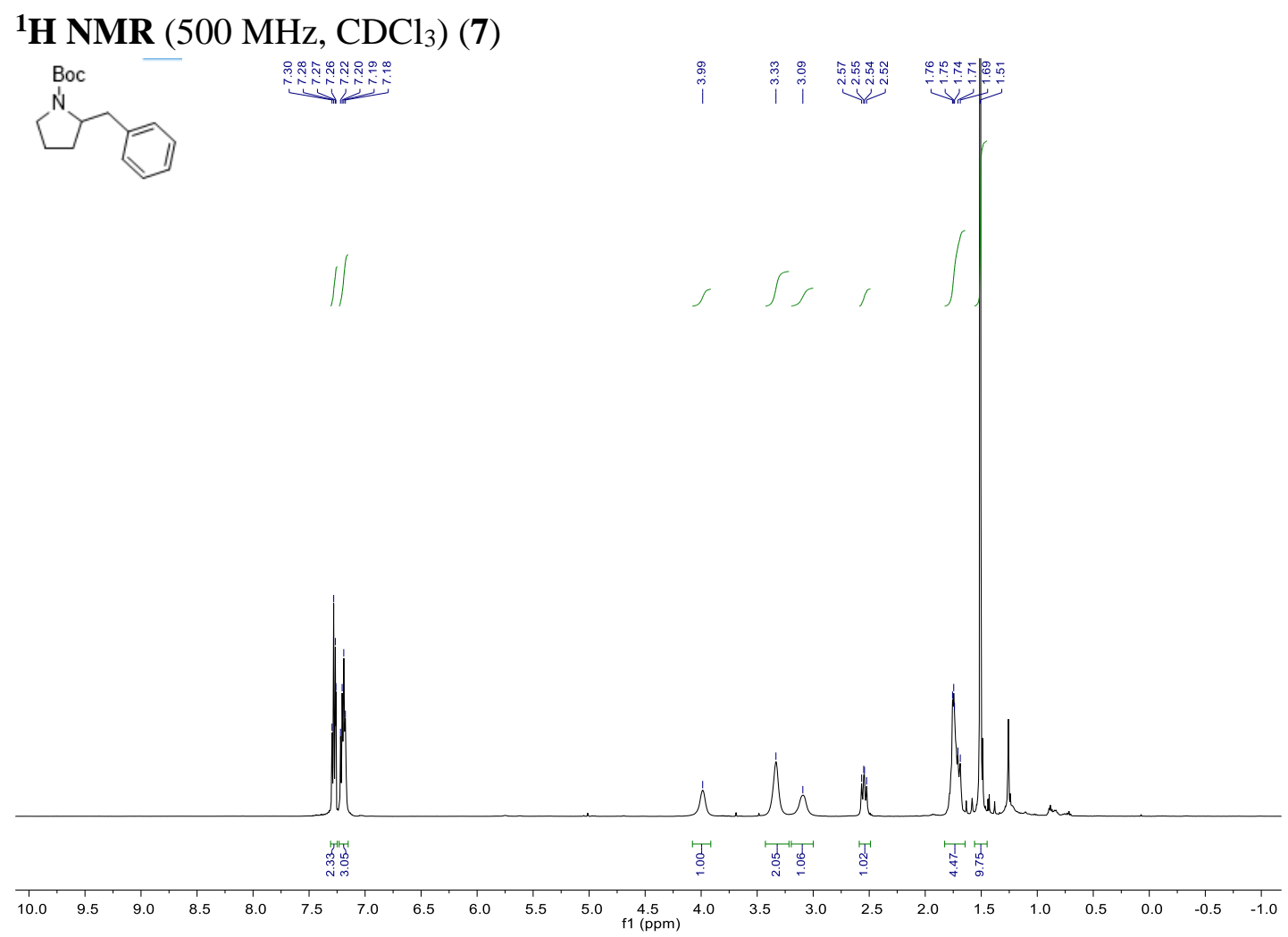

${ }^{13}$ C NMR (151 MHz, $\left.\mathrm{CDCl}_{3}\right)$ (7)
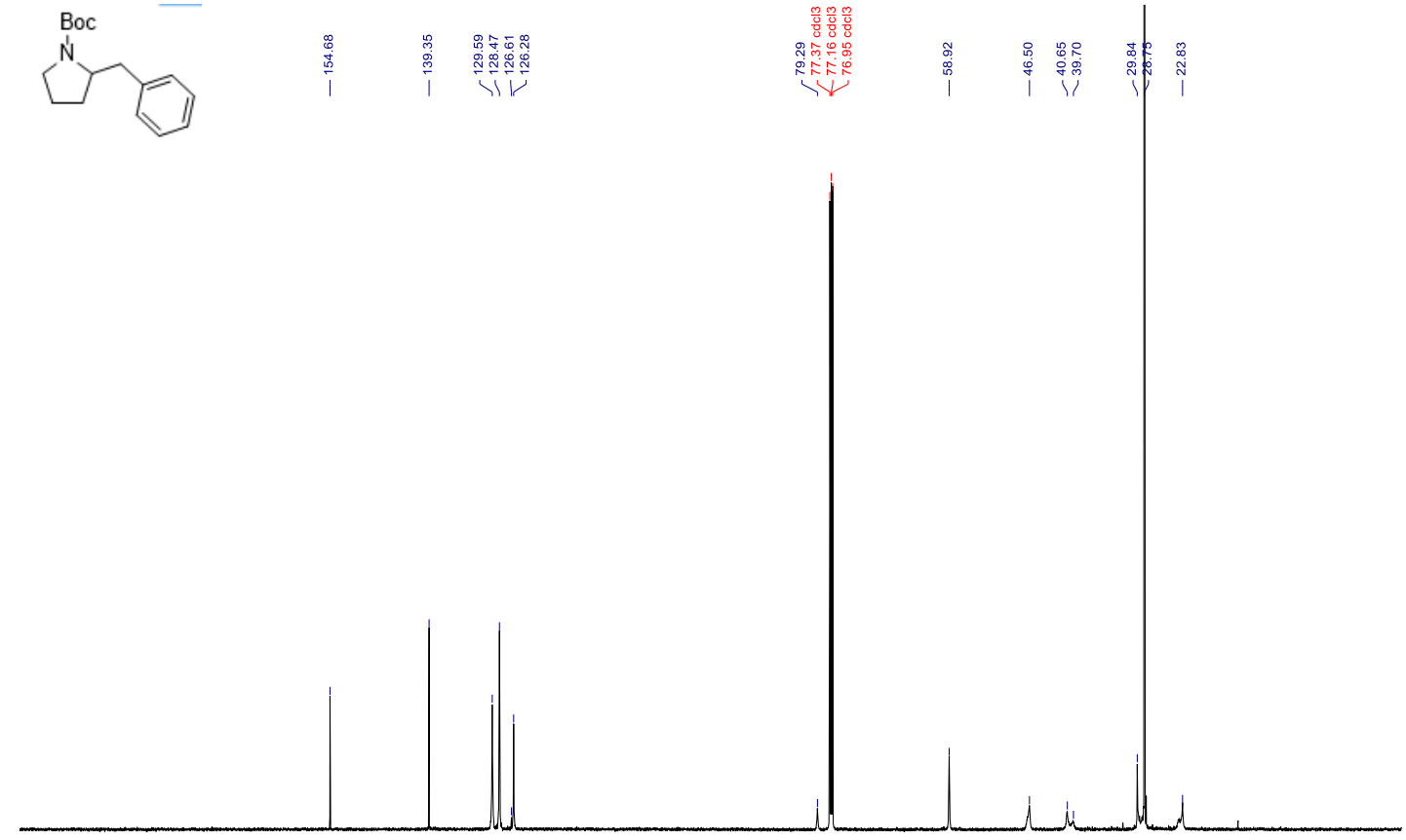

200
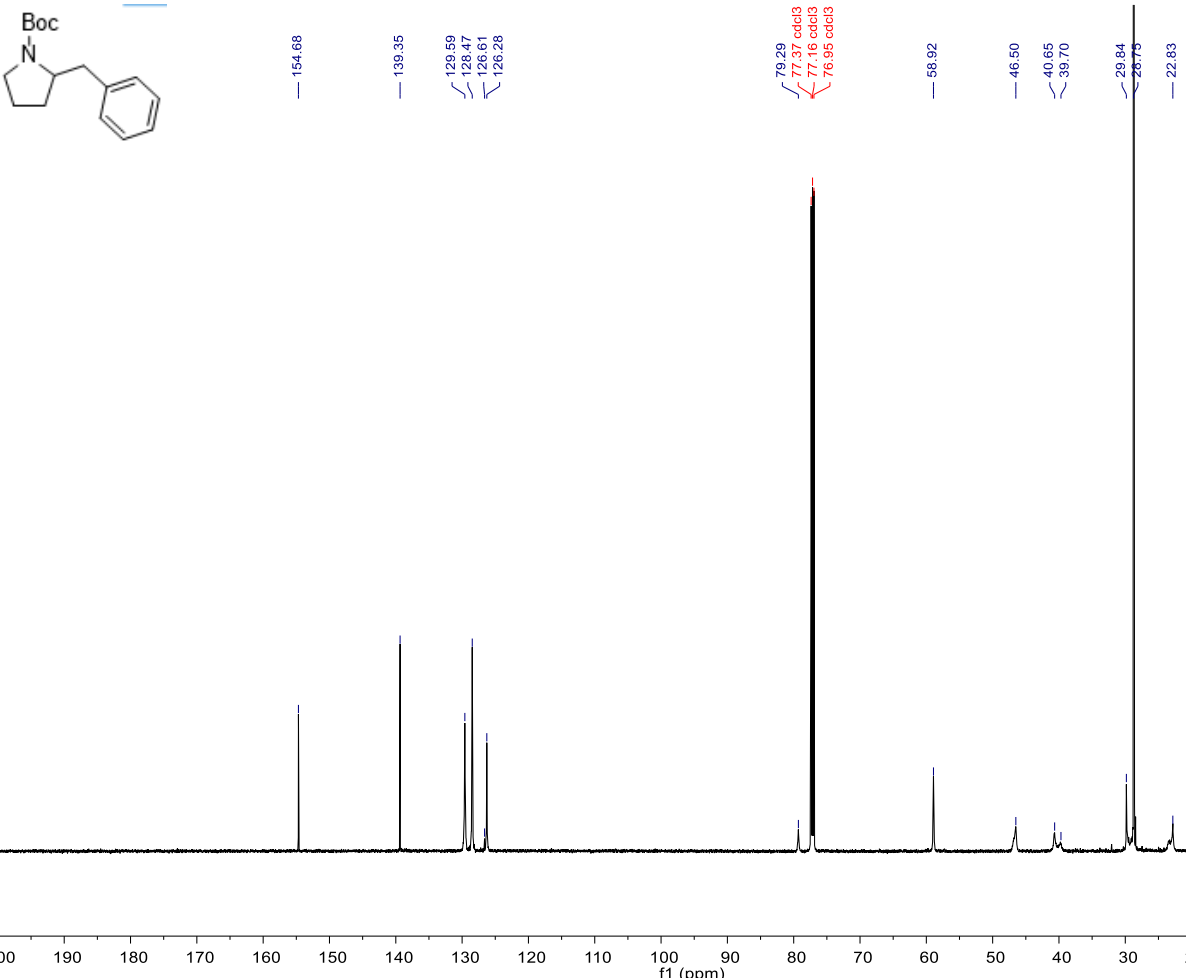


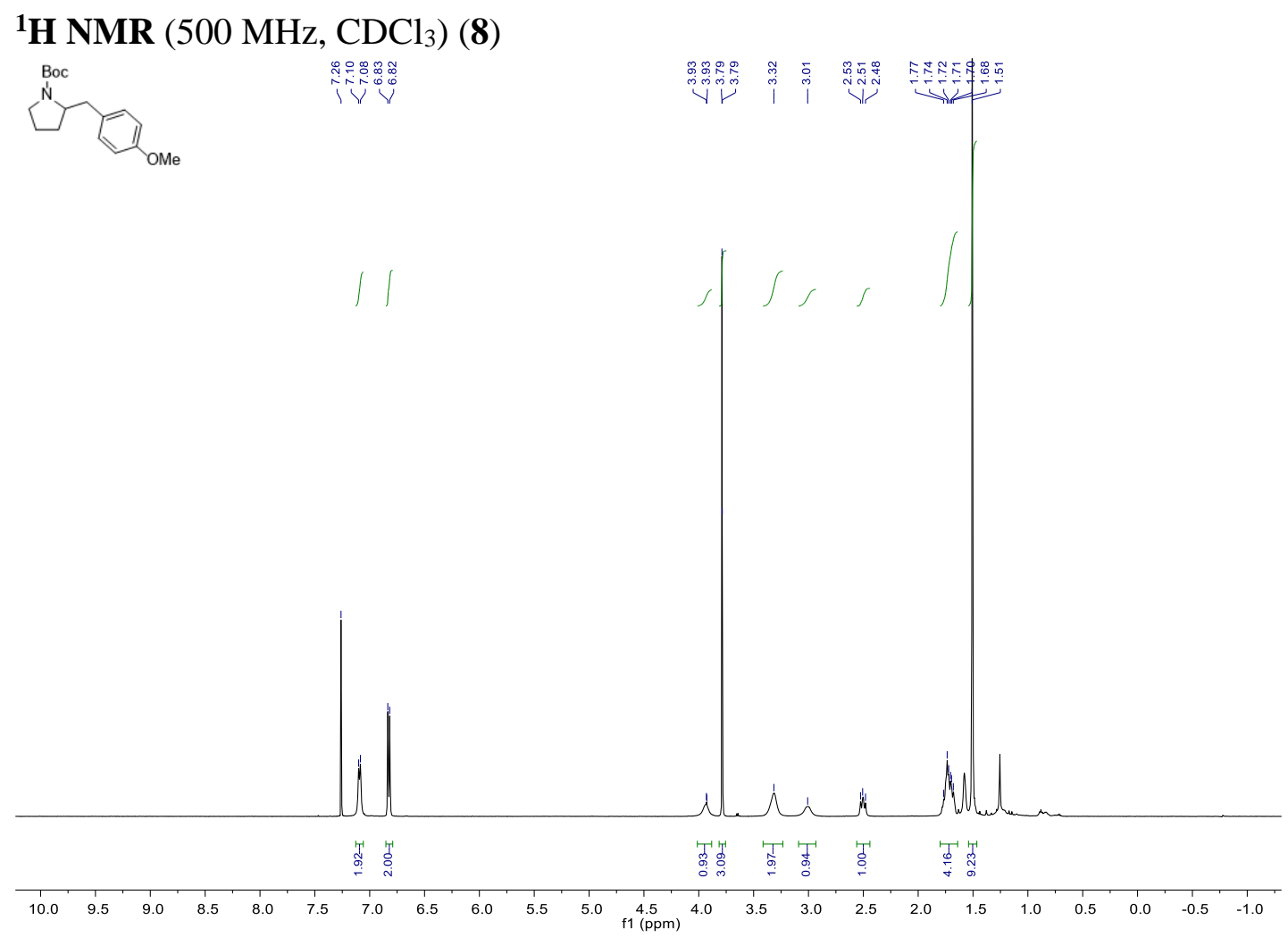

${ }^{13} \mathbf{C}$ NMR (151 MHz, $\left.\mathrm{CDCl}_{3}\right)(8)$
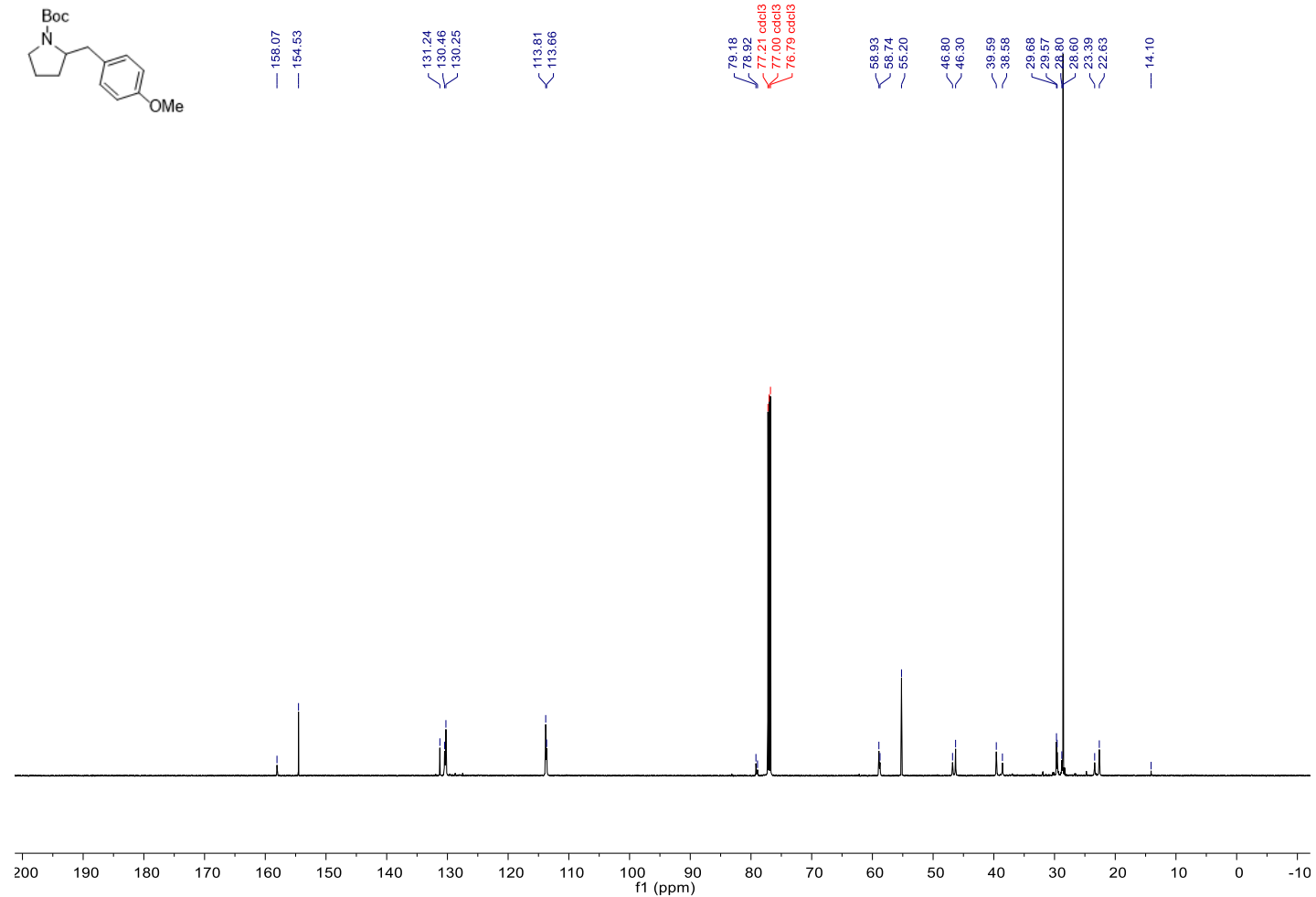


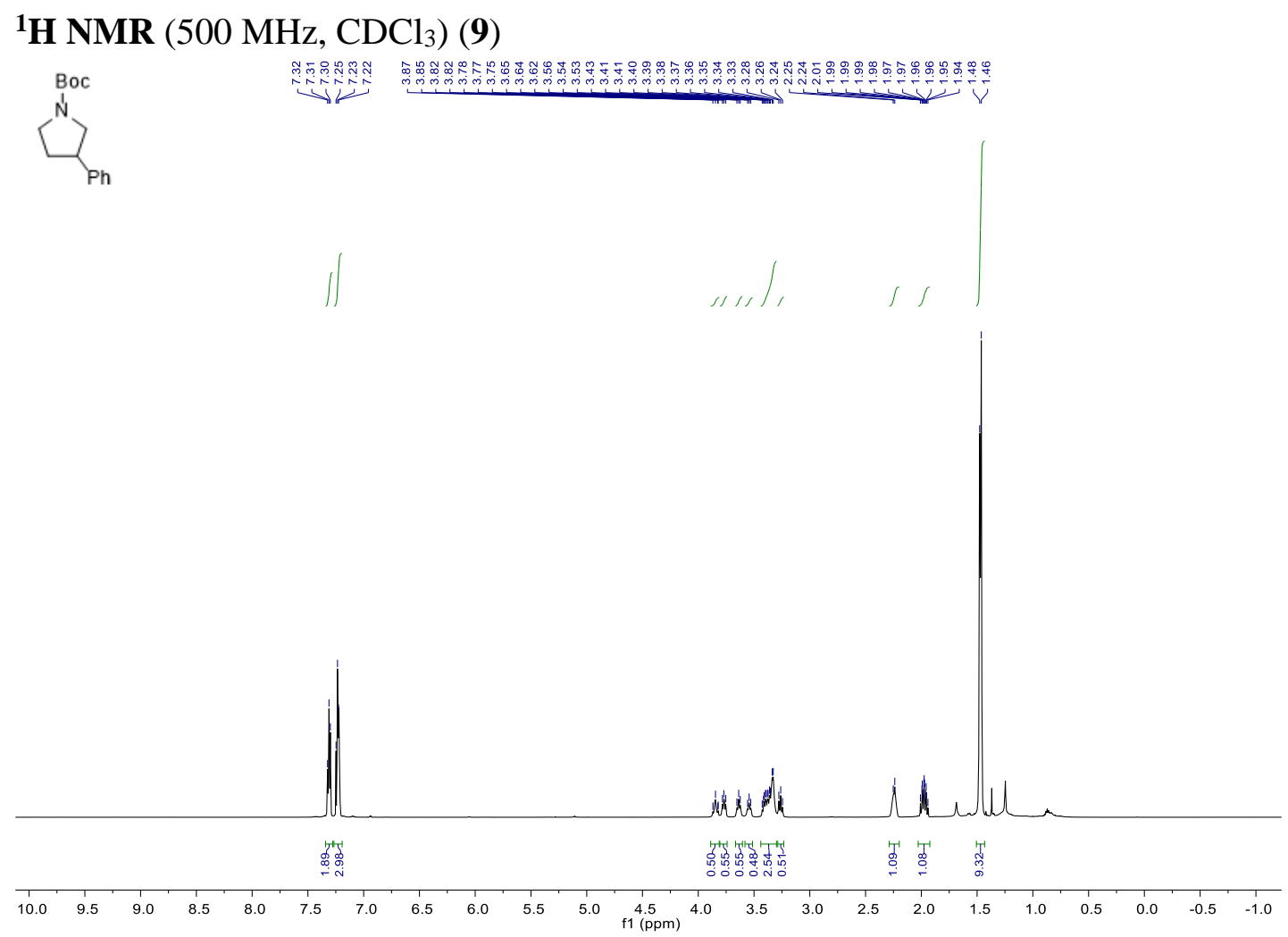

${ }^{13} \mathrm{C}$ NMR (151 MHz, $\left.\mathrm{CDCl}_{3}\right)$ (9)
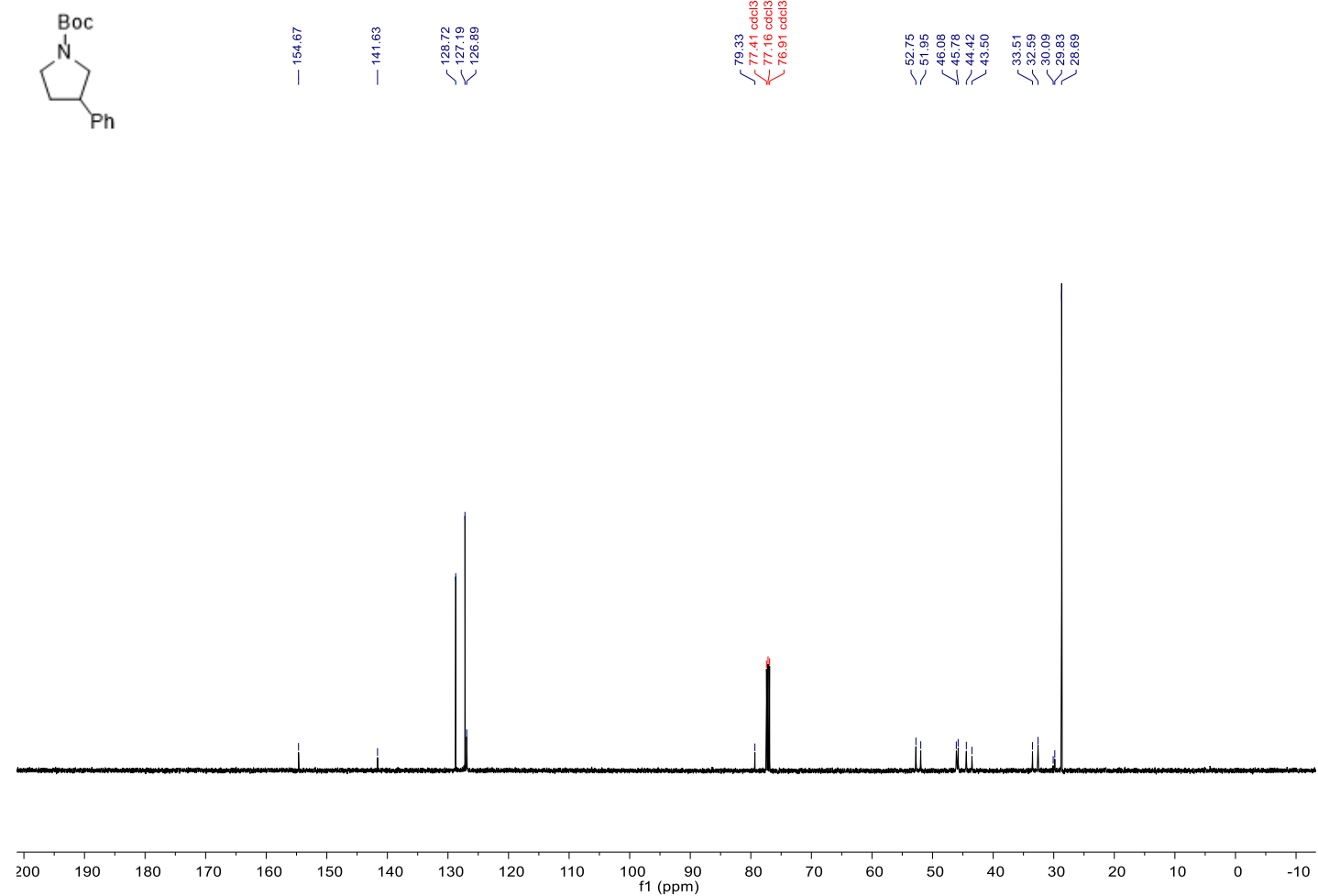
Xu, Zhang, Ingoglia, Allais, Dechert-Schmitt, Singer and Morken Supporting Information

${ }^{1} \mathbf{H}$ NMR (500 MHz, $\left.\mathrm{CDCl}_{3}\right)(\mathbf{1 0})$

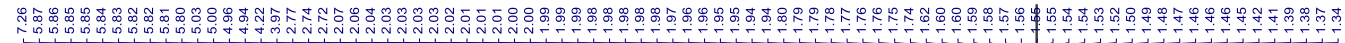
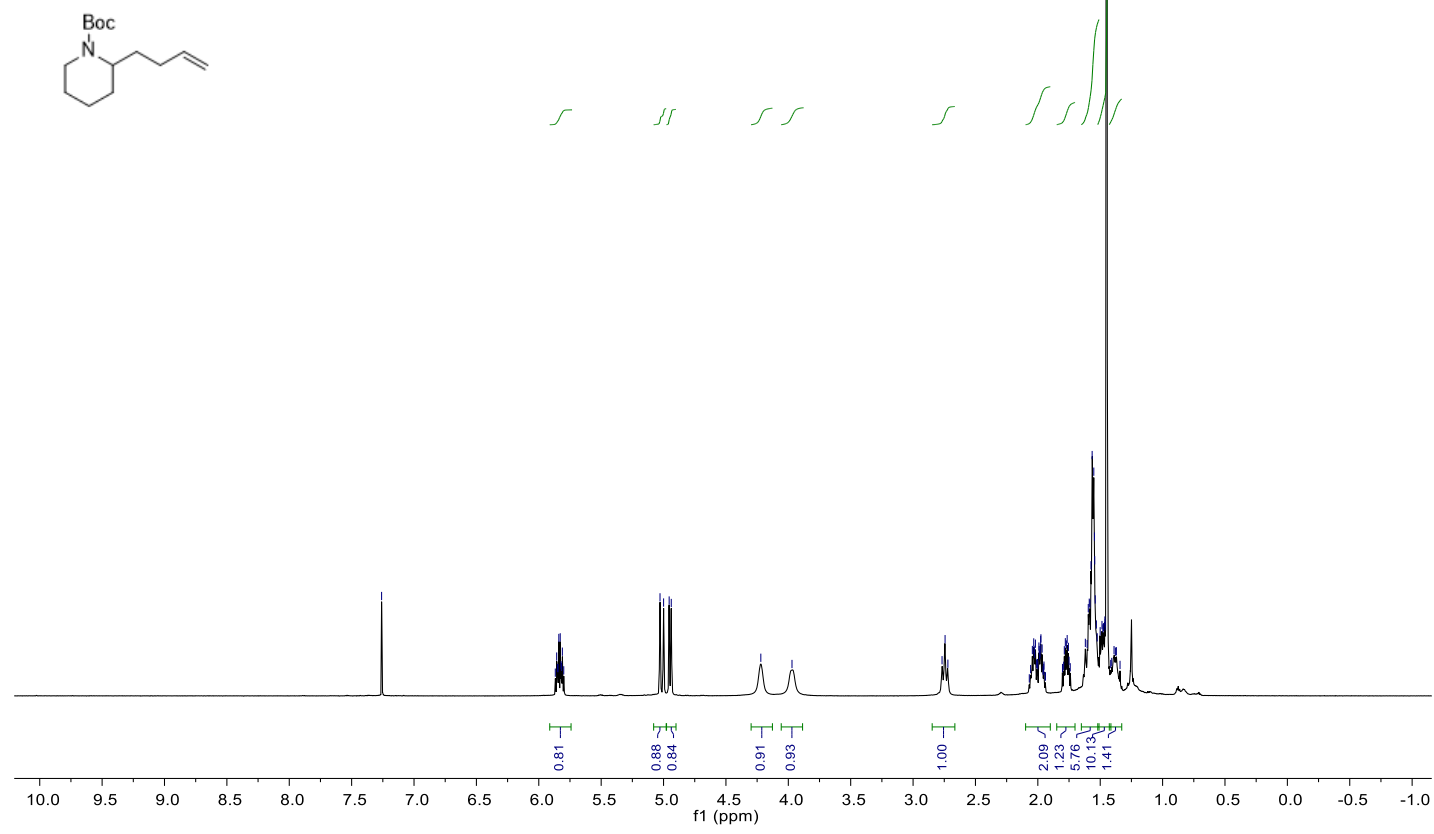

${ }^{13}$ C NMR (151 MHz, $\left.\mathrm{CDCl}_{3}\right)$ (10)
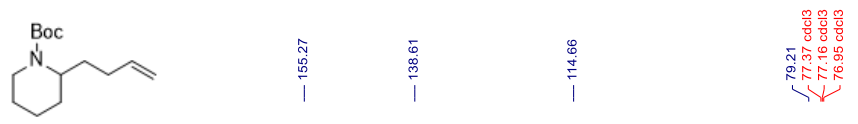

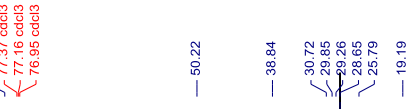
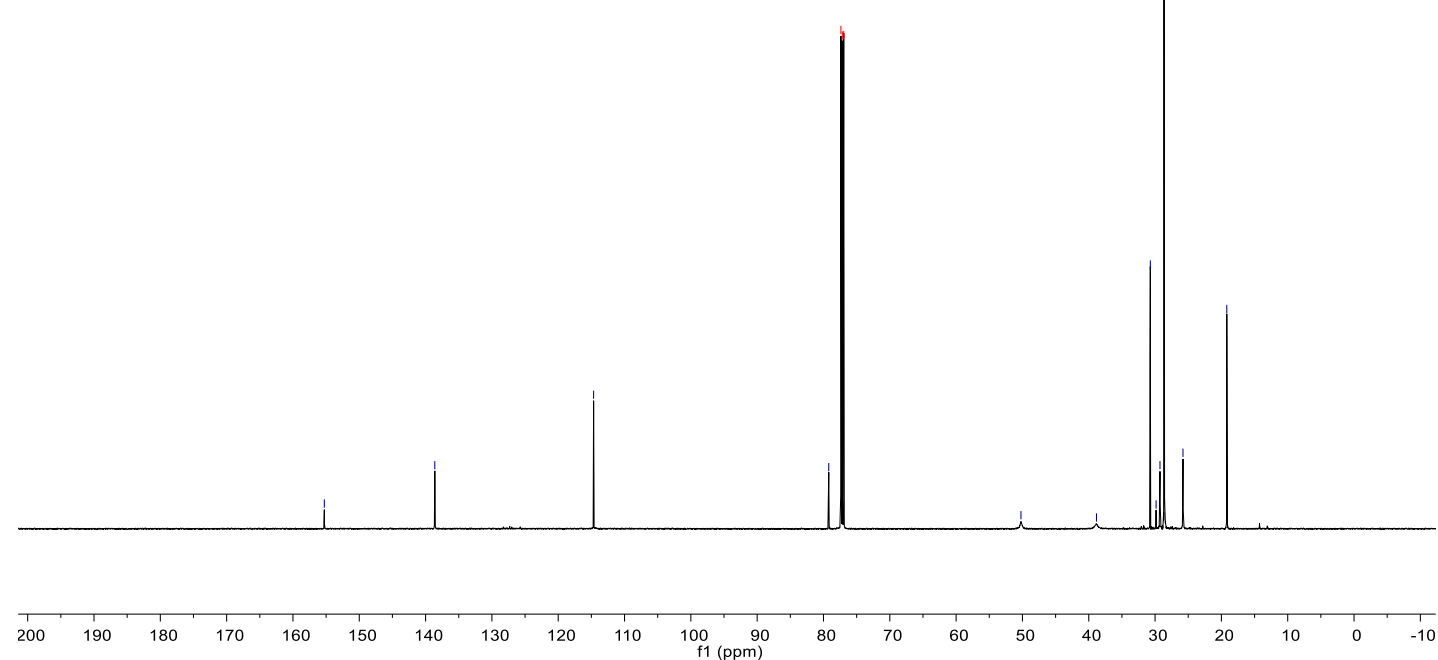


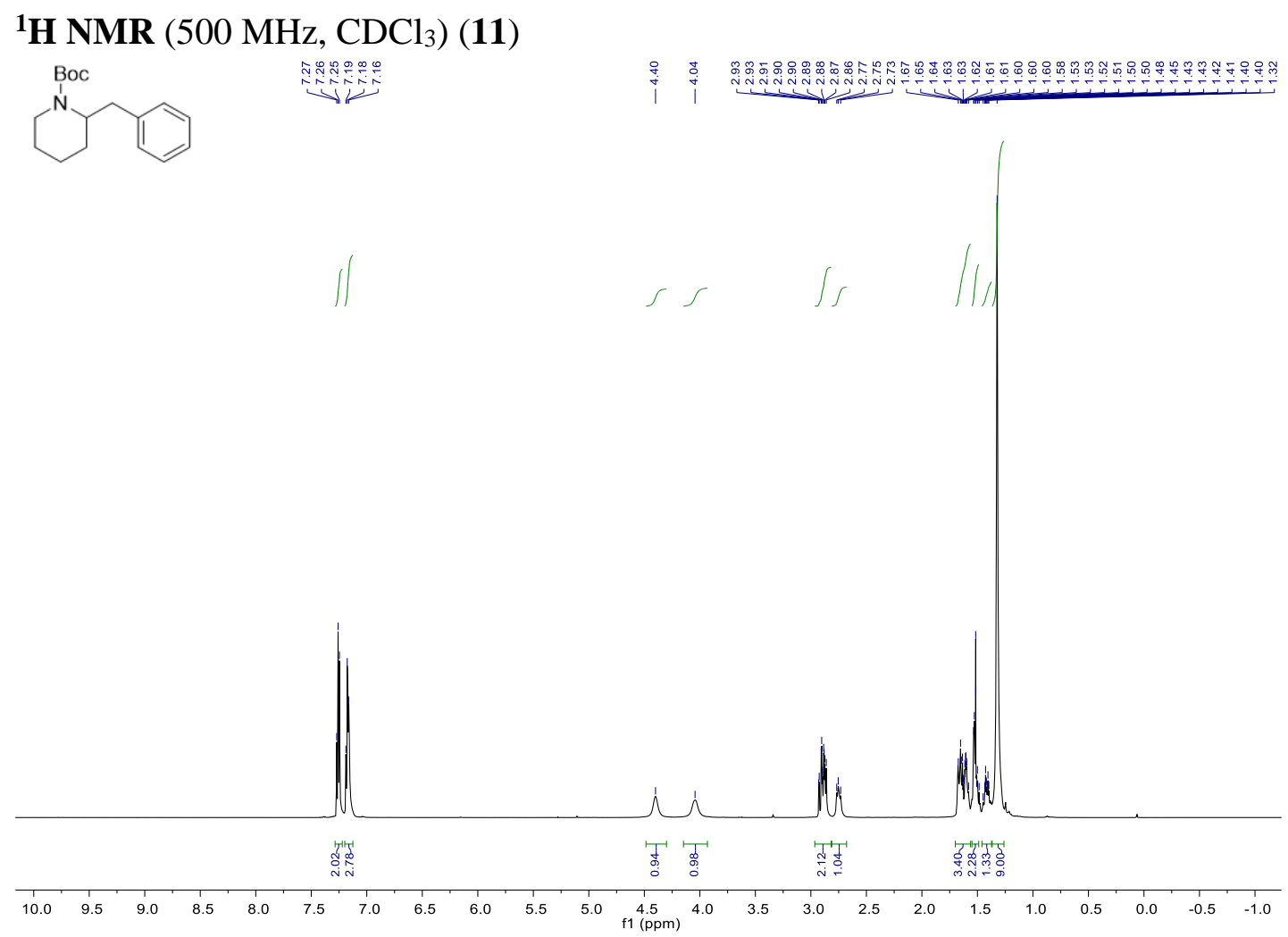

${ }^{13}$ C NMR (151 MHz, $\left.\mathrm{CDCl}_{3}\right)$ (11)
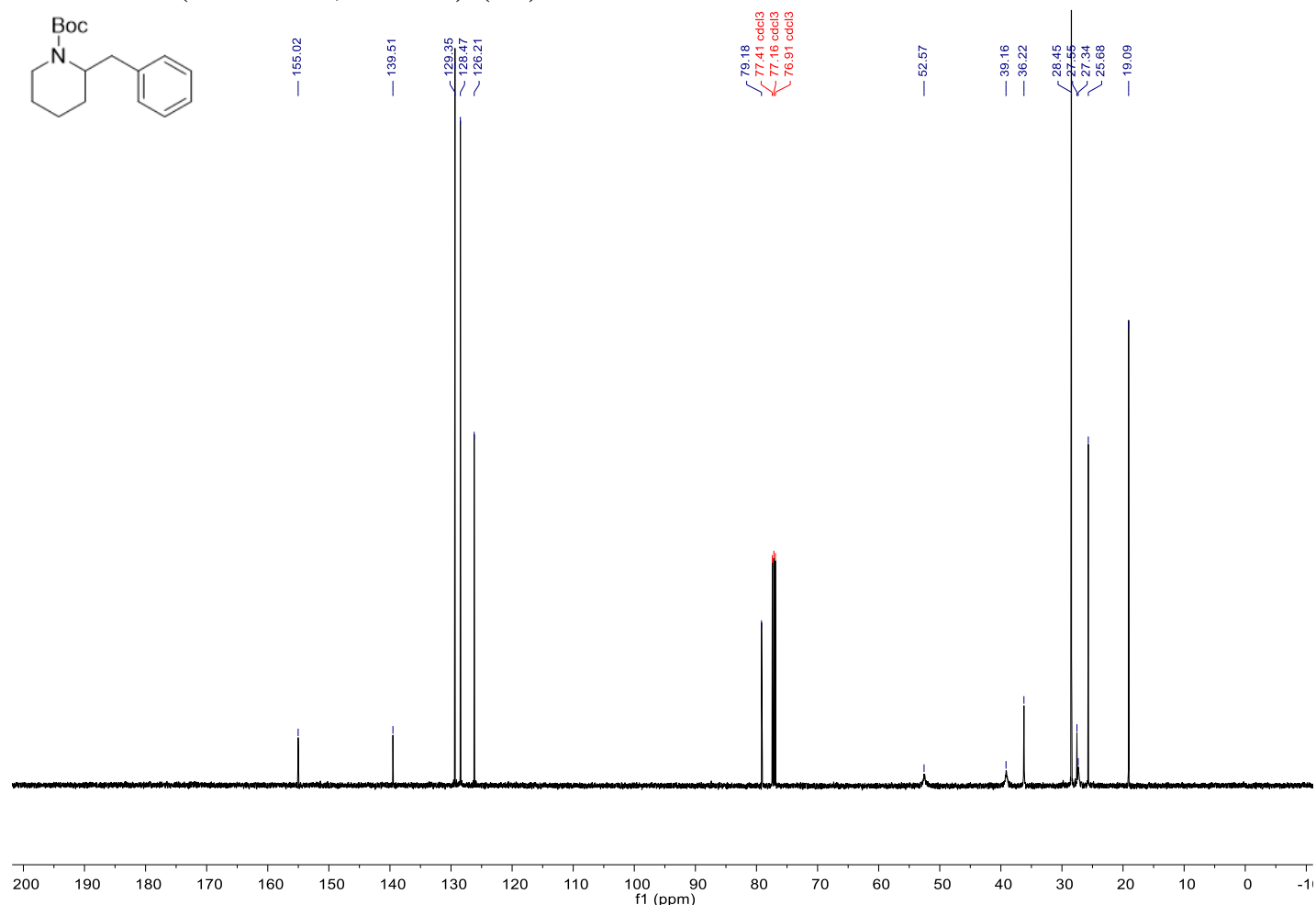


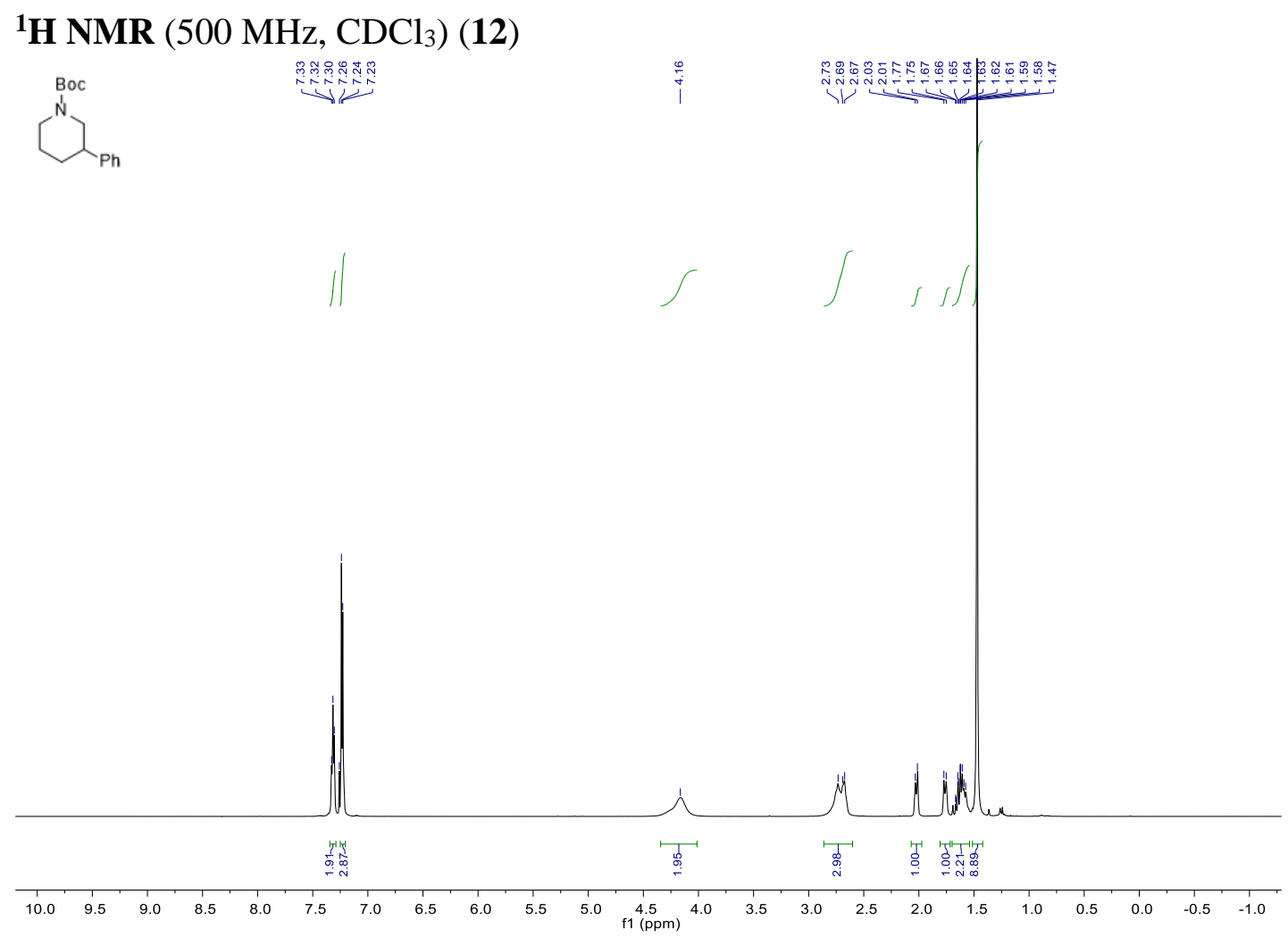

${ }^{13}$ C NMR (151 MHz, $\left.\mathrm{CDCl}_{3}\right)$ (12)
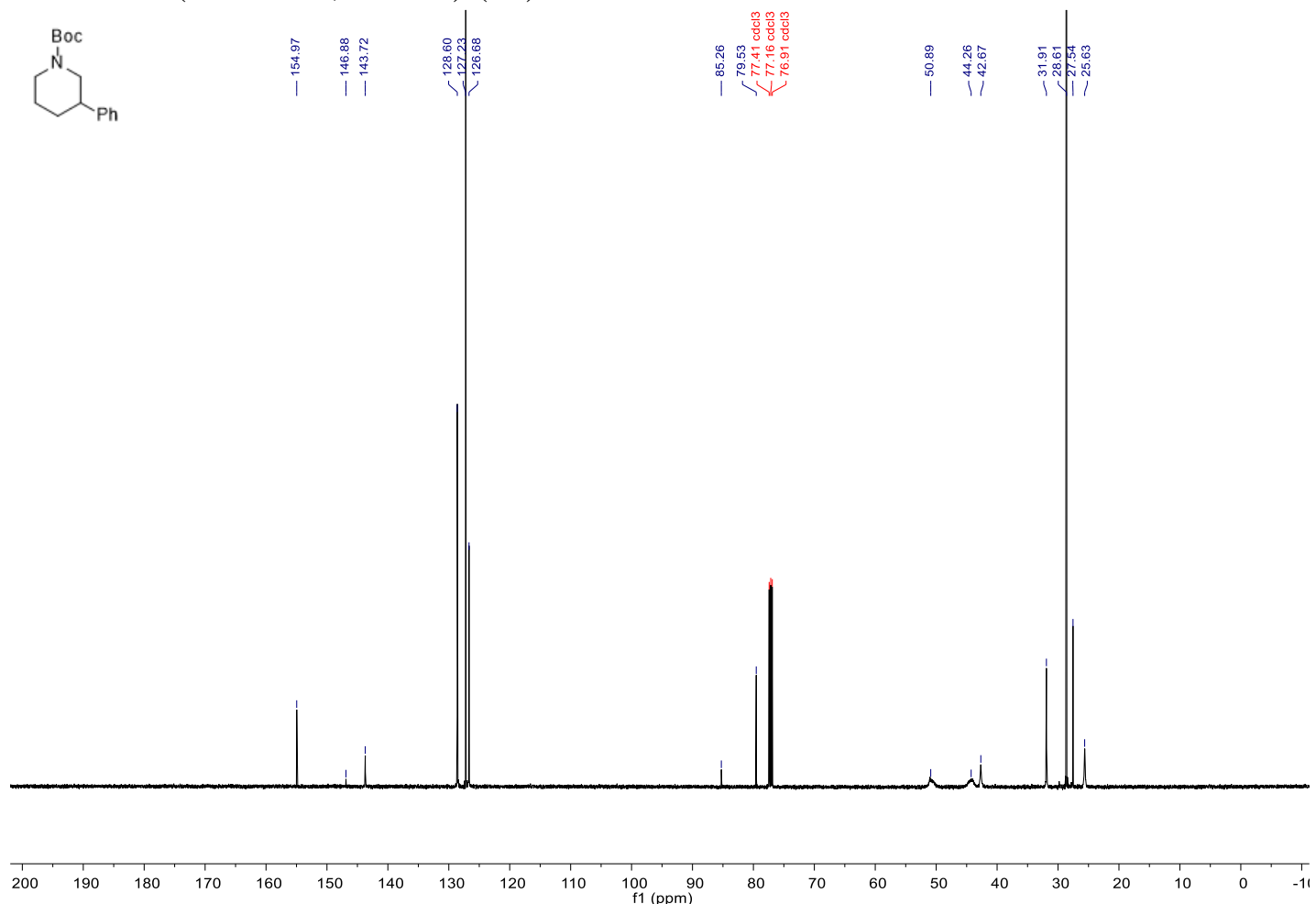
Xu, Zhang, Ingoglia, Allais, Dechert-Schmitt, Singer and Morken Supporting Information

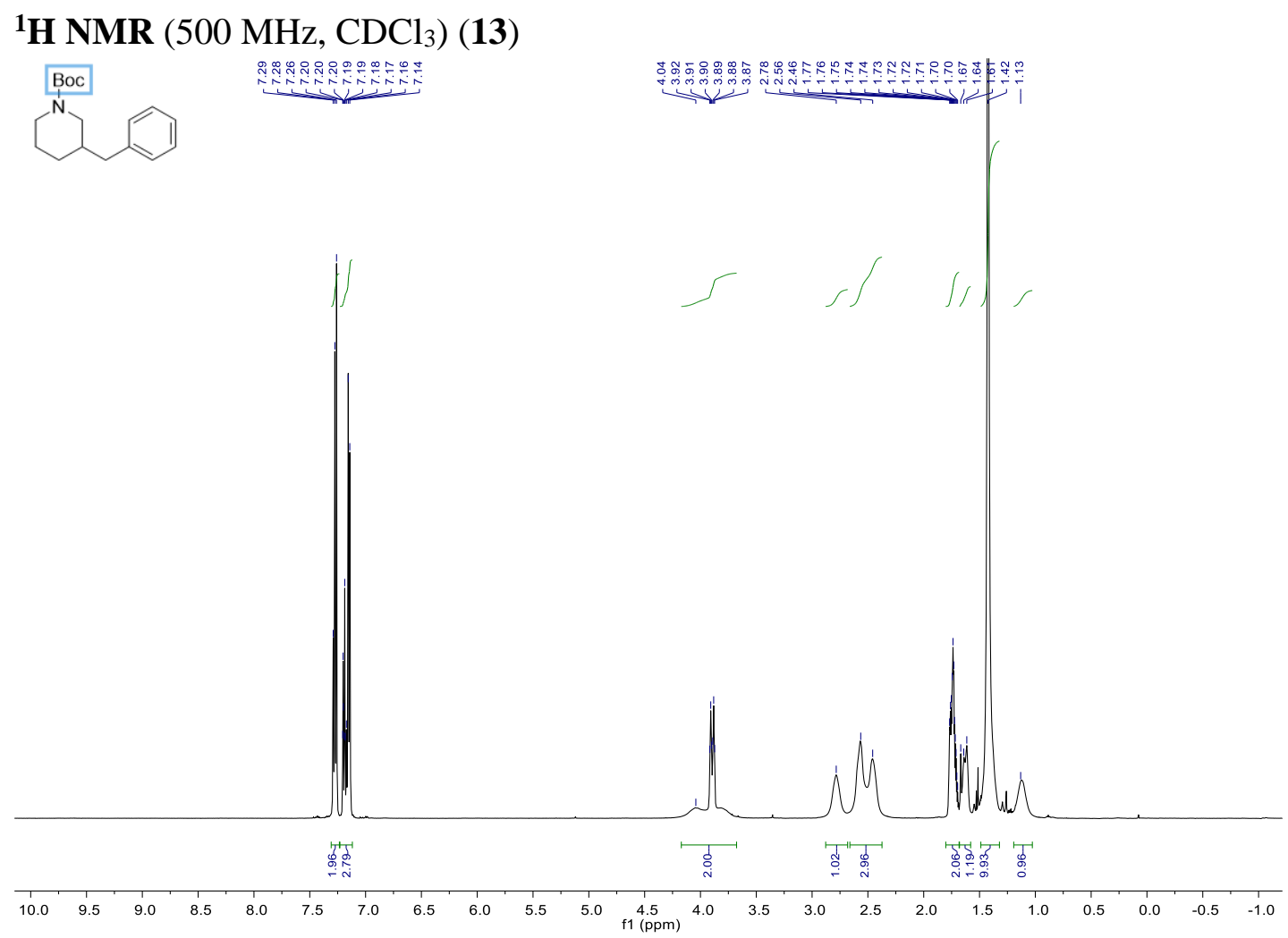

${ }^{13}$ C NMR (151 MHz, $\left.\mathrm{CDCl}_{3}\right)(\mathbf{1 3})$
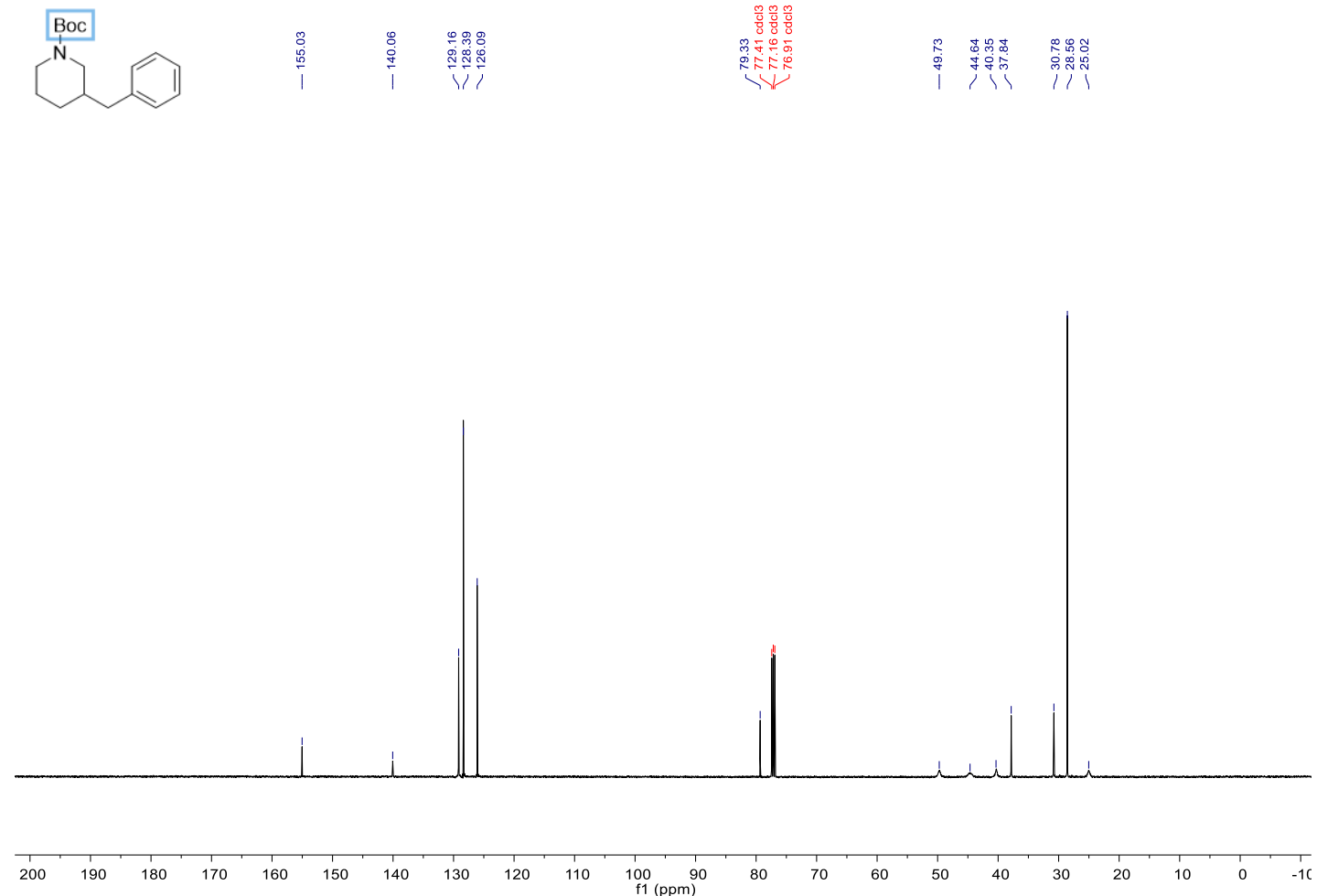


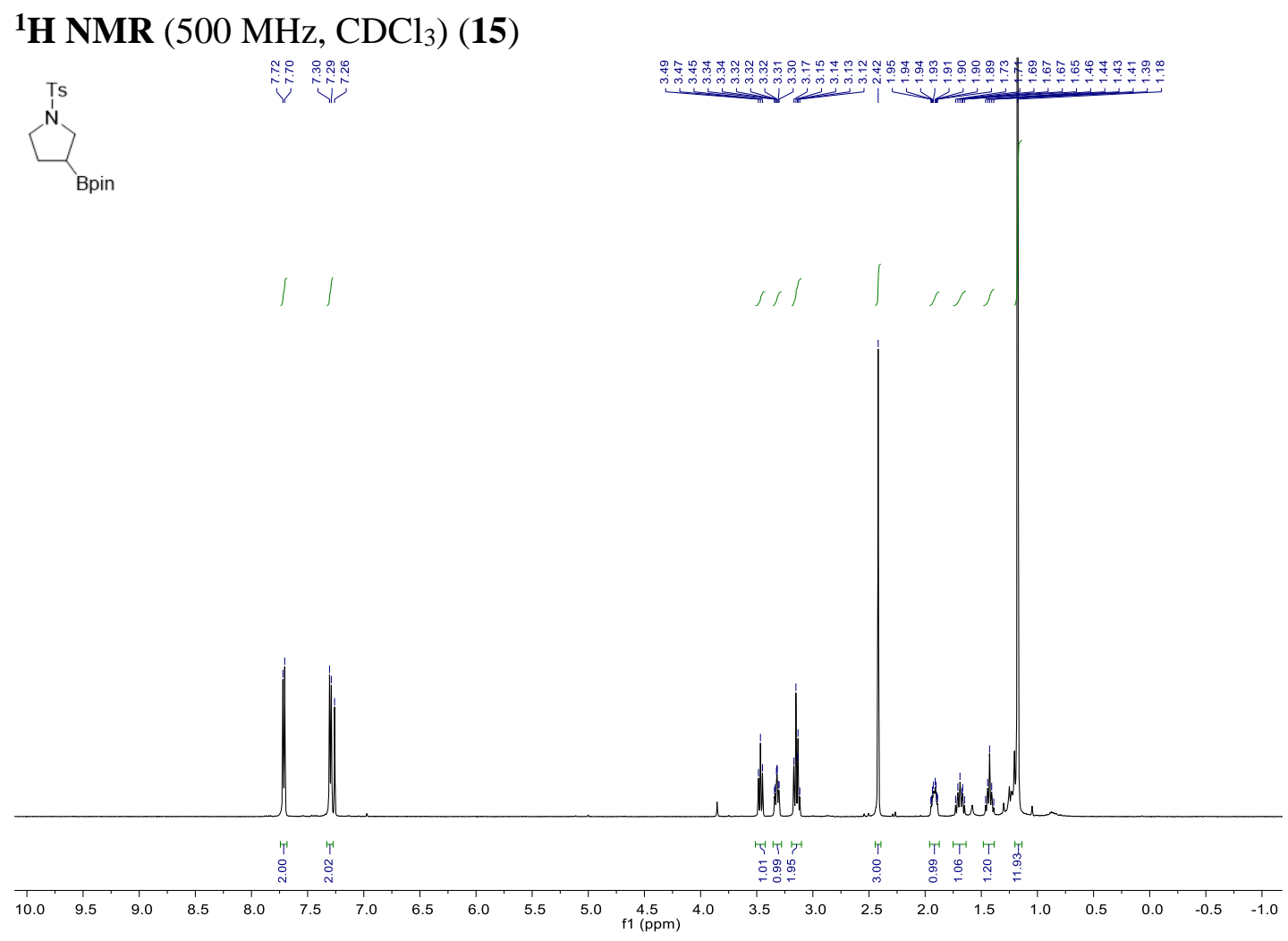

${ }^{13} \mathbf{C}$ NMR (126 MHz, $\left.\mathrm{CDCl}_{3}\right)$ (15)
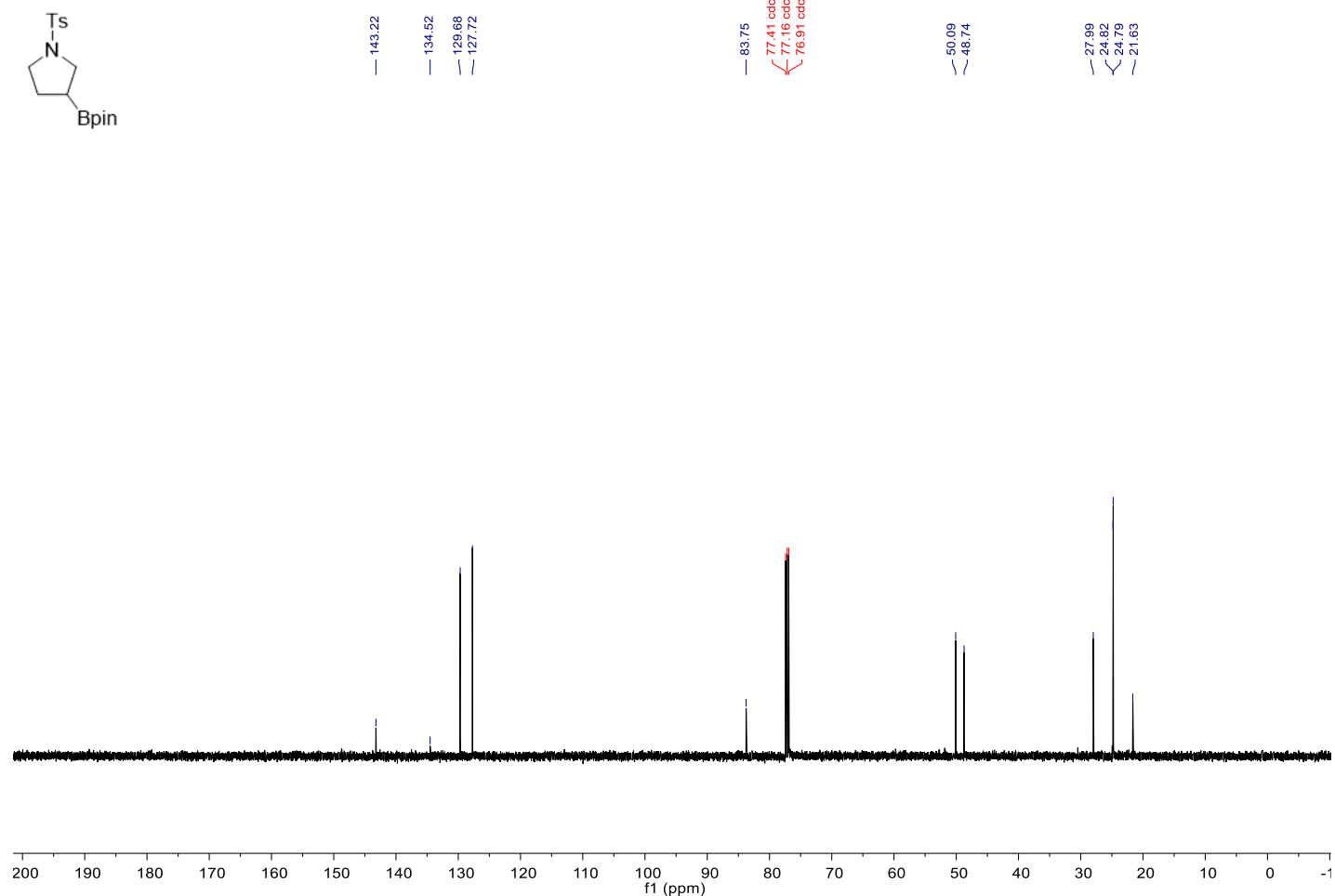
Xu, Zhang, Ingoglia, Allais, Dechert-Schmitt, Singer and Morken Supporting Information

${ }^{\mathbf{1}} \mathbf{H}$ NMR (500 MHz, $\left.\mathrm{CDCl}_{3}\right)(\mathbf{1 7})$
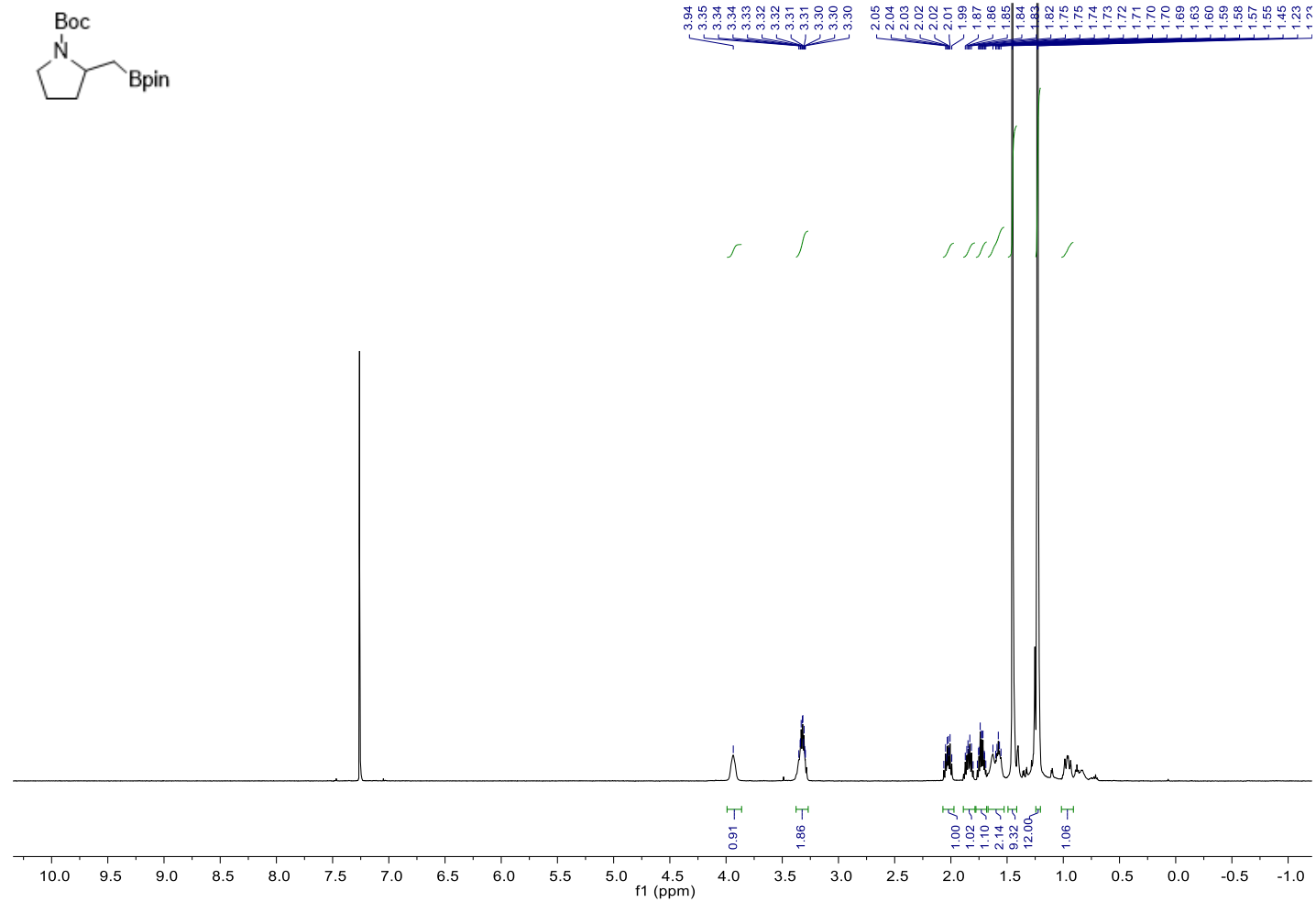

${ }^{13}$ C NMR (151 MHz, $\left.\mathrm{CDCl}_{3}\right)$ (17)

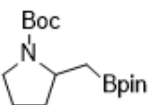

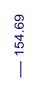
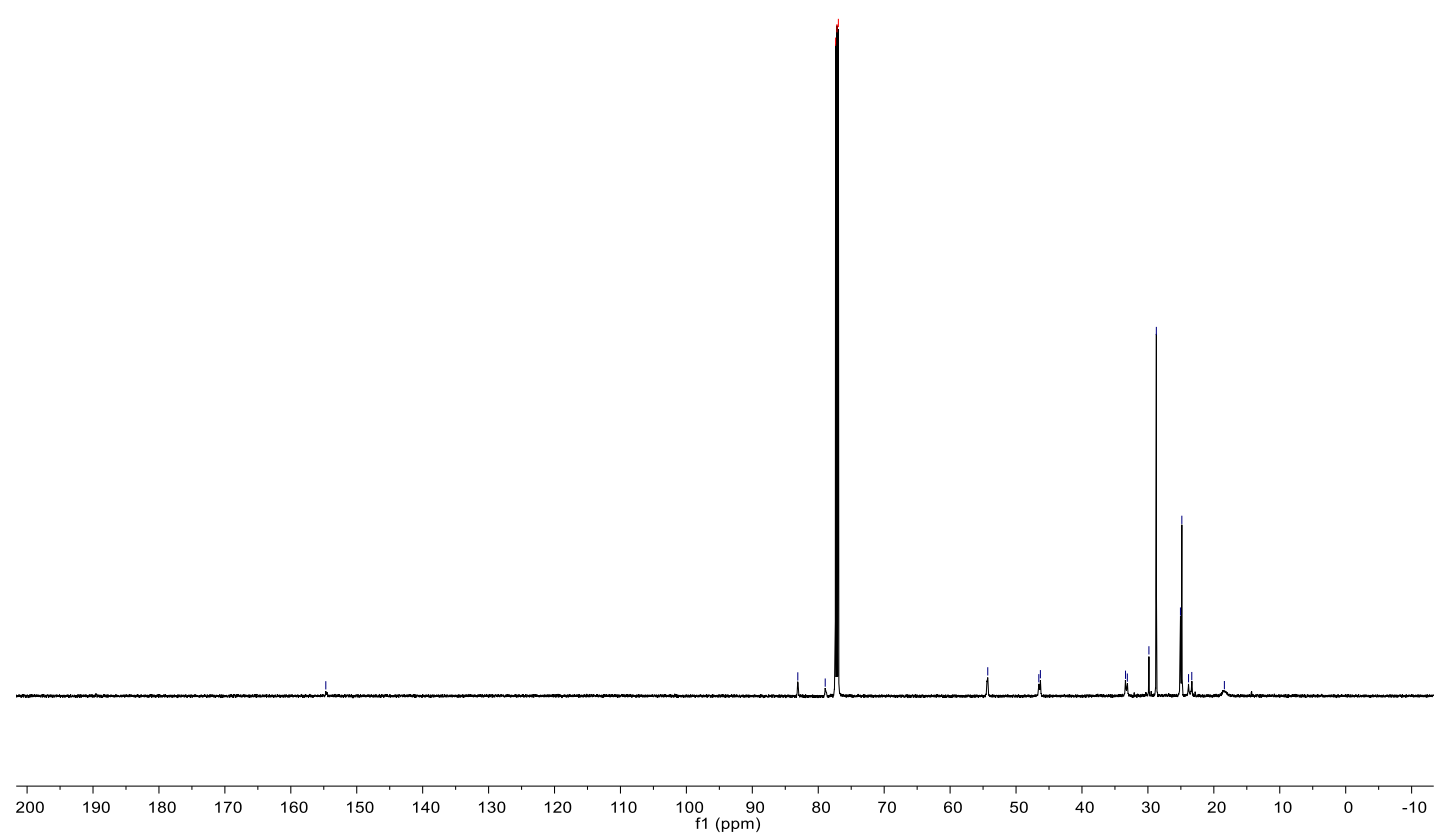
Xu, Zhang, Ingoglia, Allais, Dechert-Schmitt, Singer and Morken Supporting Information

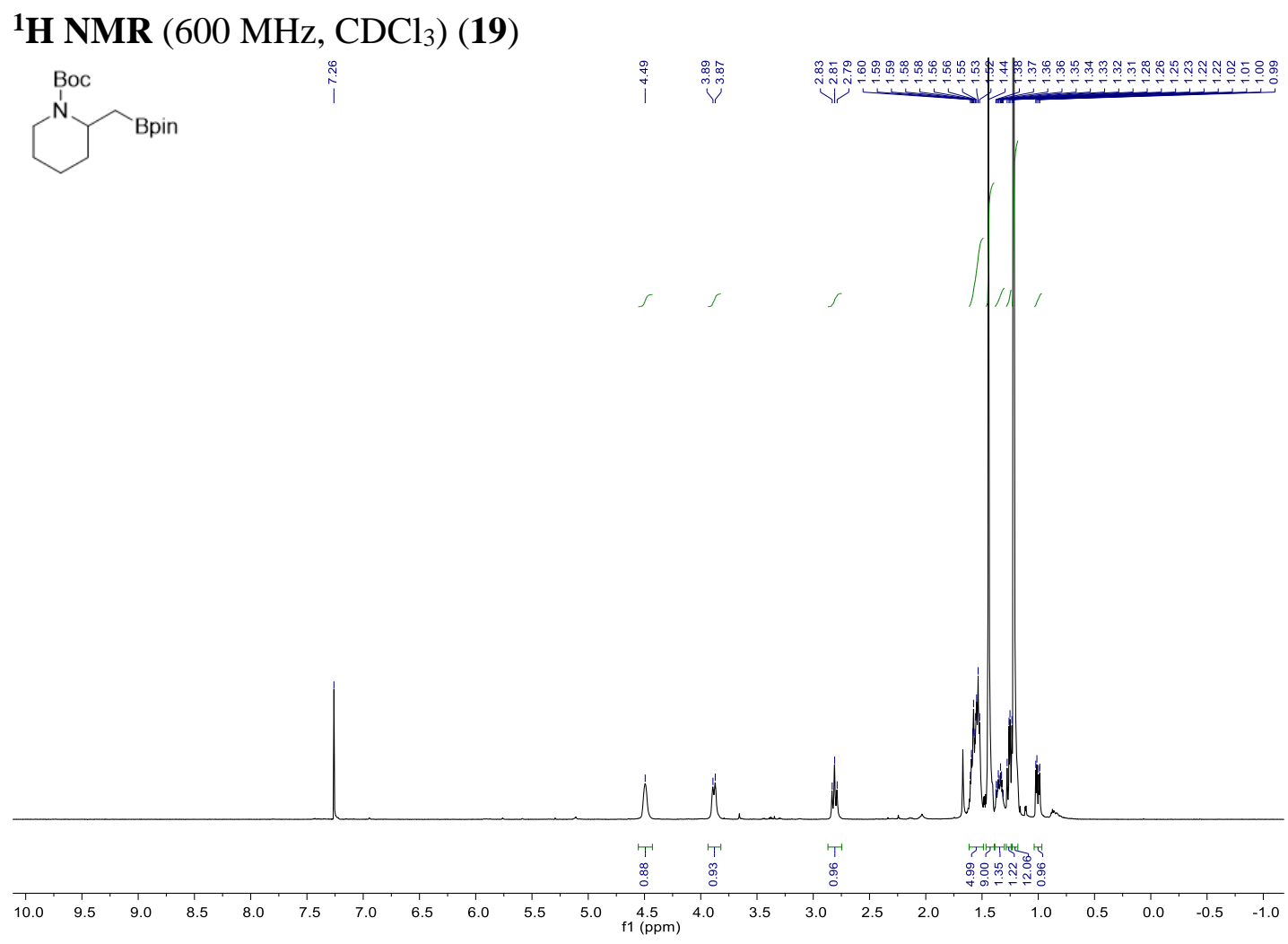

${ }^{13} \mathbf{C ~ N M R}\left(126 \mathrm{MHz}, \mathrm{CDCl}_{3}\right)$ (19)
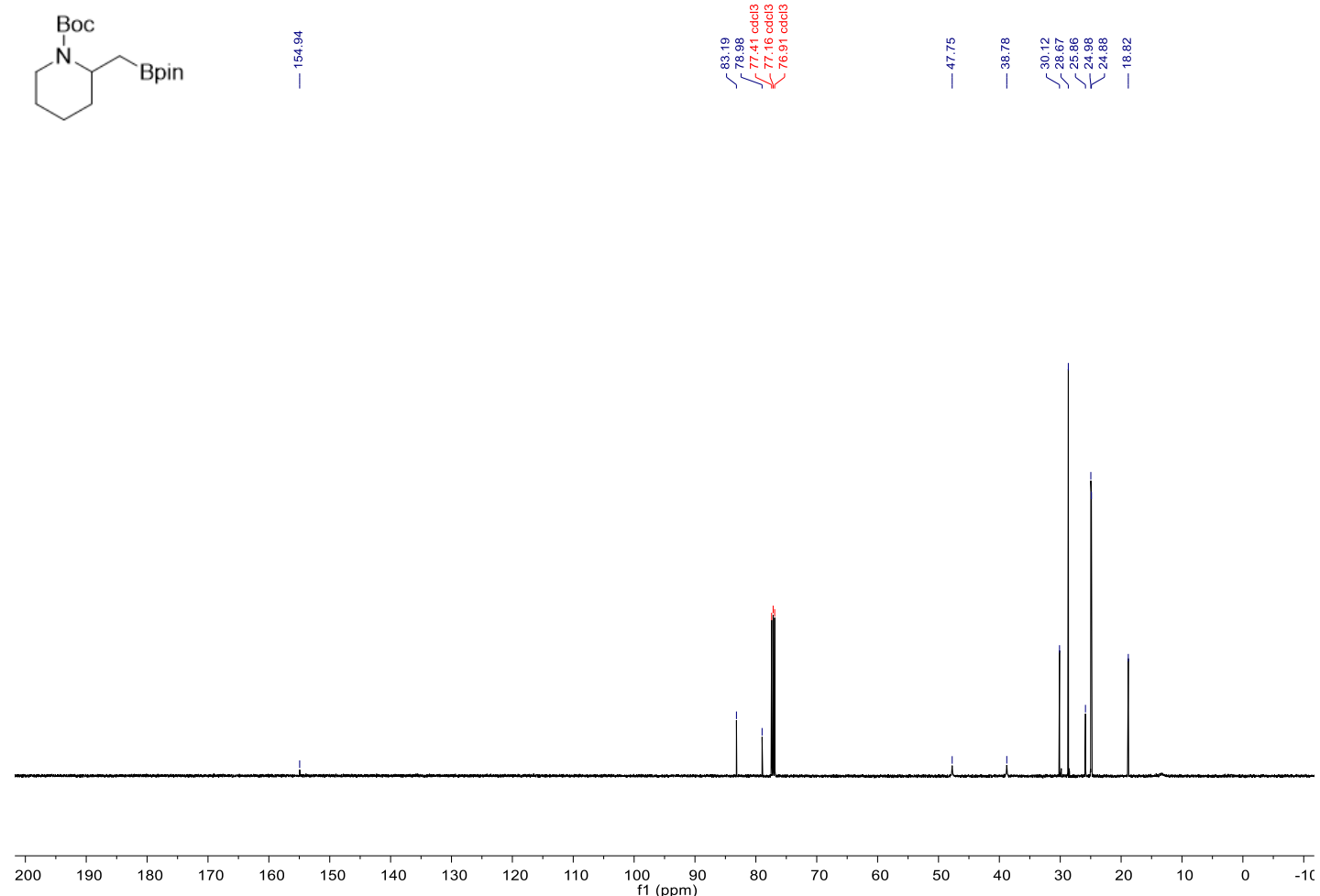
Xu, Zhang, Ingoglia, Allais, Dechert-Schmitt, Singer and Morken Supporting Information

${ }^{\mathbf{1}} \mathbf{H}$ NMR $\left(500 \mathrm{MHz}, \mathrm{CDCl}_{3}\right)(\mathbf{3 1})$<smiles>CC(C)[C@H]1CCN1</smiles>

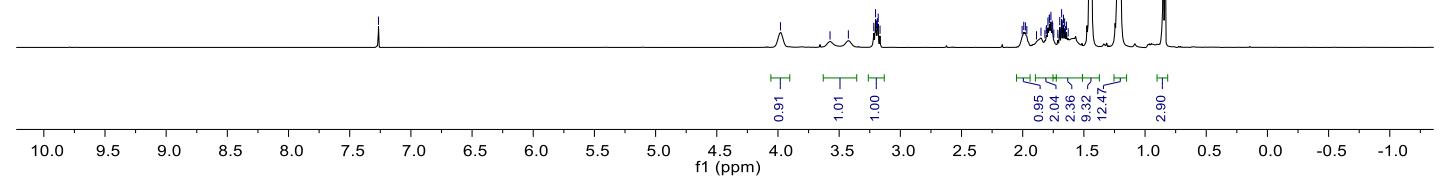

${ }^{13} \mathbf{C}$ NMR (151 MHz, $\left.\mathrm{CDCl}_{3}\right)$ (31)<smiles>CCC(C)C1CO1</smiles> 


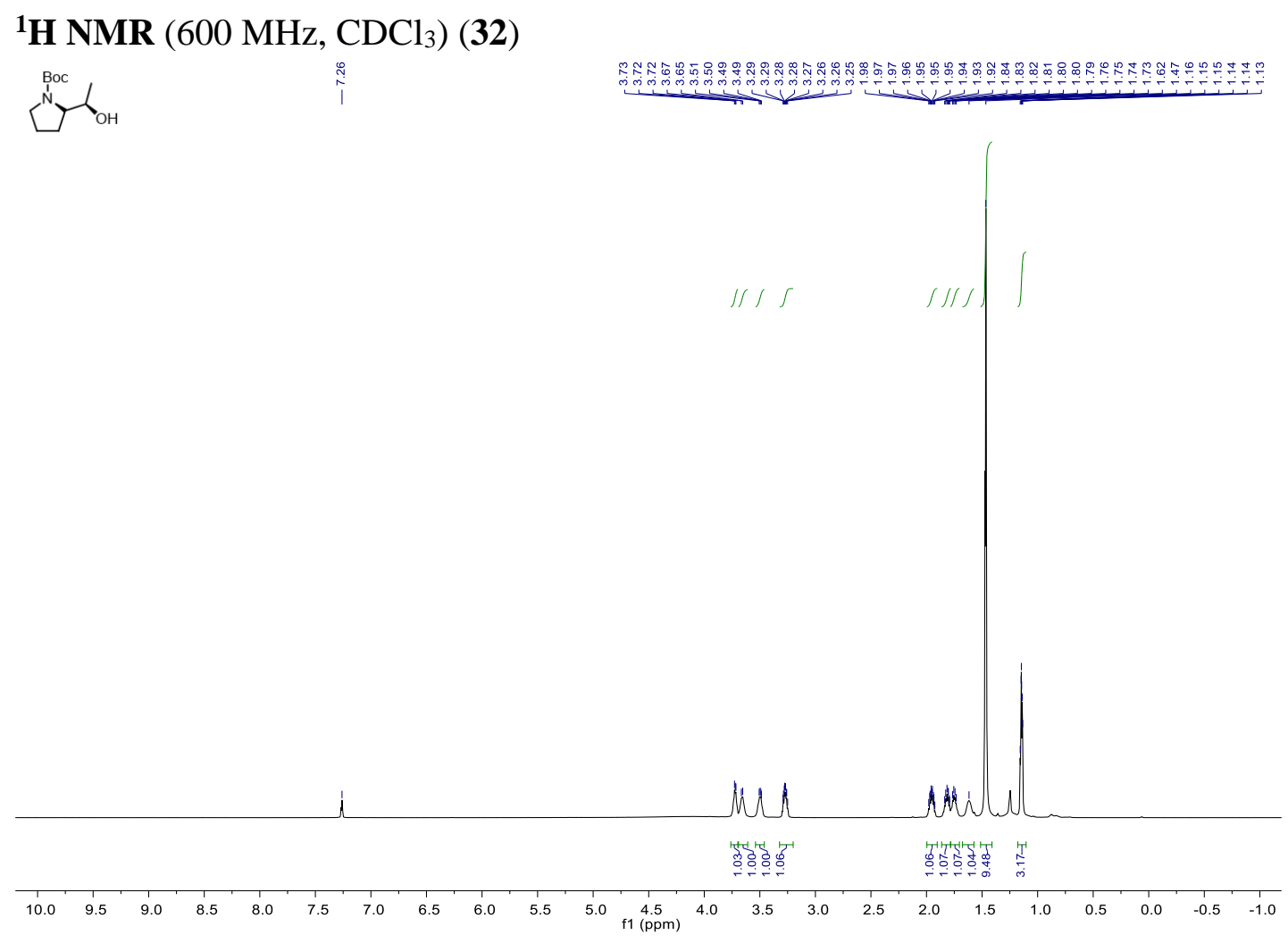

${ }^{13}$ C NMR (151 MHz, $\left.\mathrm{CDCl}_{3}\right)$ (32)
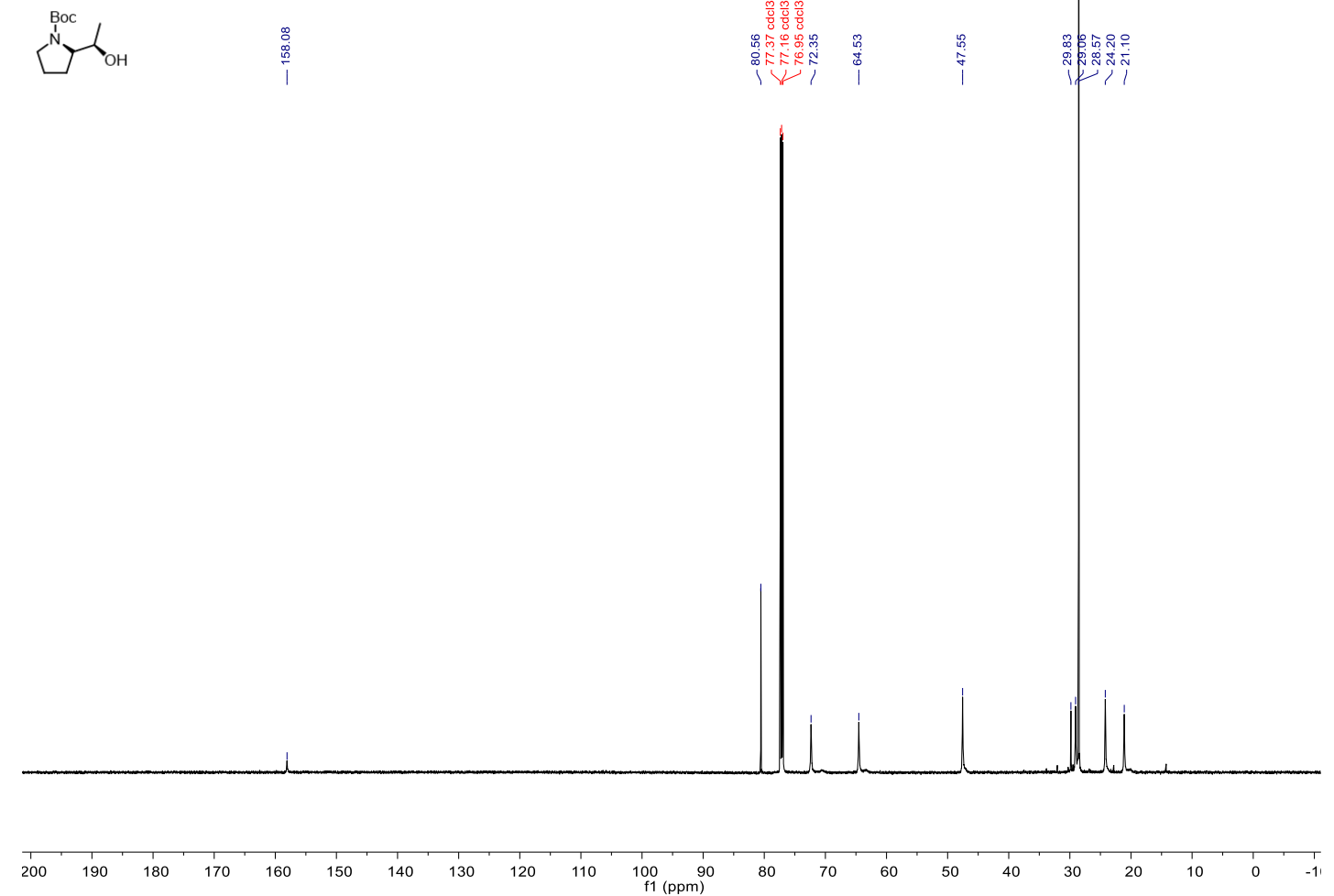
Xu, Zhang, Ingoglia, Allais, Dechert-Schmitt, Singer and Morken Supporting Information

${ }^{\mathbf{1}} \mathbf{H} \mathbf{N M R}\left(600 \mathrm{MHz}, \mathrm{CDCl}_{3}\right)(\mathbf{2 1})$

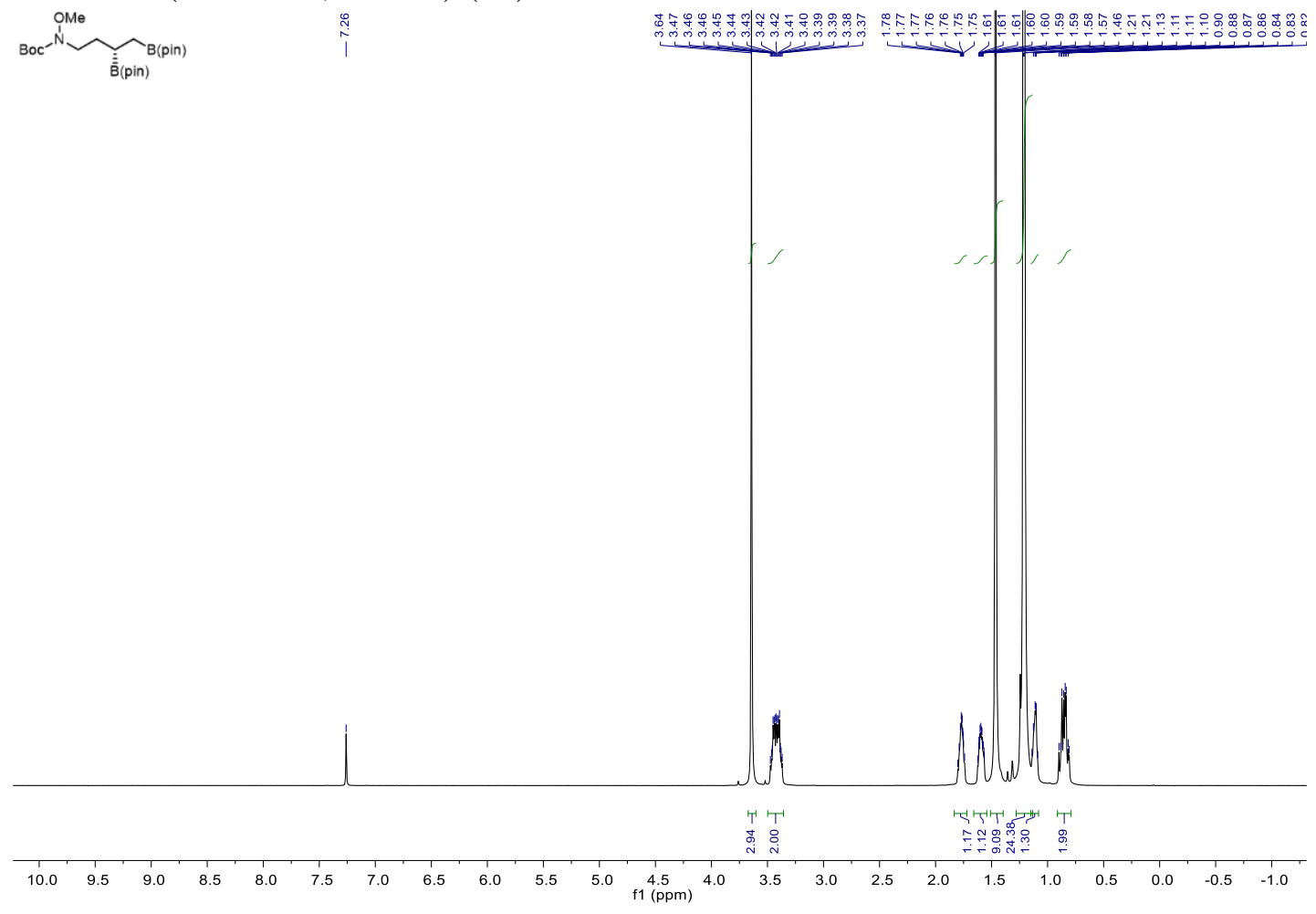

${ }^{13}$ C NMR (151 MHz, $\left.\mathrm{CDCl}_{3}\right)$ (21)
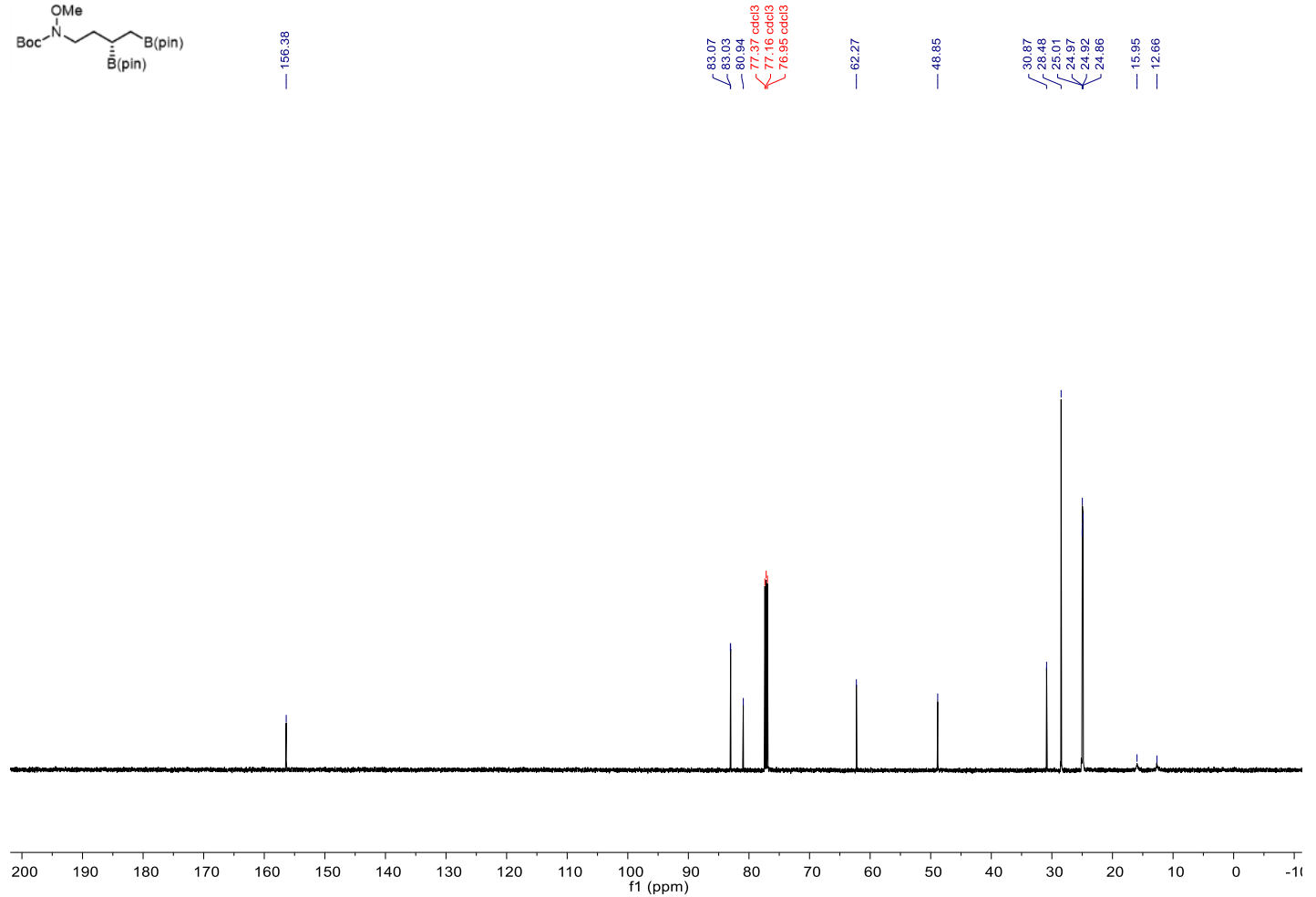


\section{${ }^{1} \mathbf{H}$ NMR (600 MHz, $\left.\mathrm{CDCl}_{3}\right)(\mathbf{2 6})$}

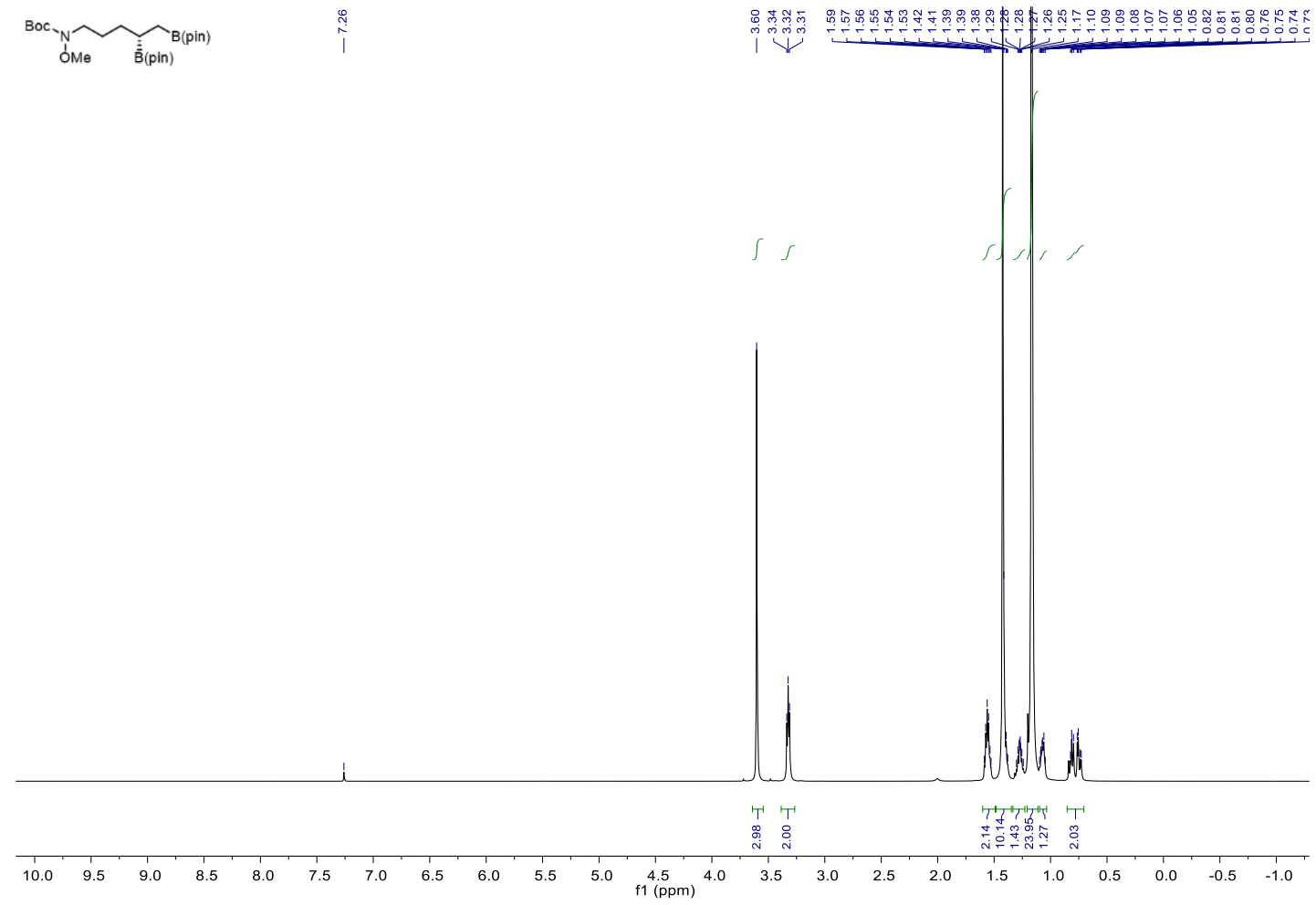

${ }^{13}$ C NMR (151 MHz, $\left.\mathrm{CDCl}_{3}\right)$ (26)
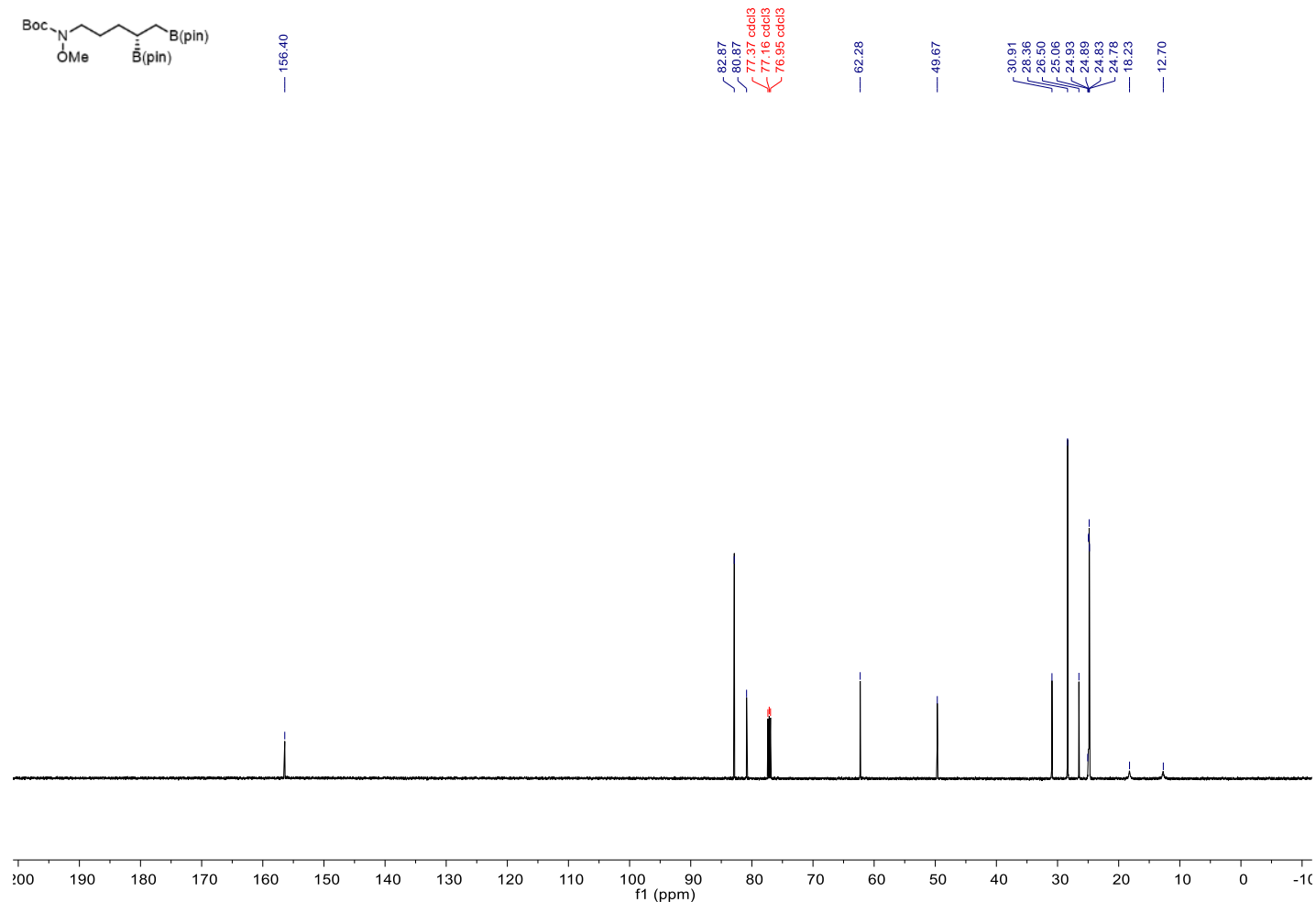
${ }^{\mathbf{1}} \mathbf{H}$ NMR (600 MHz, $\left.\mathrm{CDCl}_{3}\right)(\mathbf{2 3})$

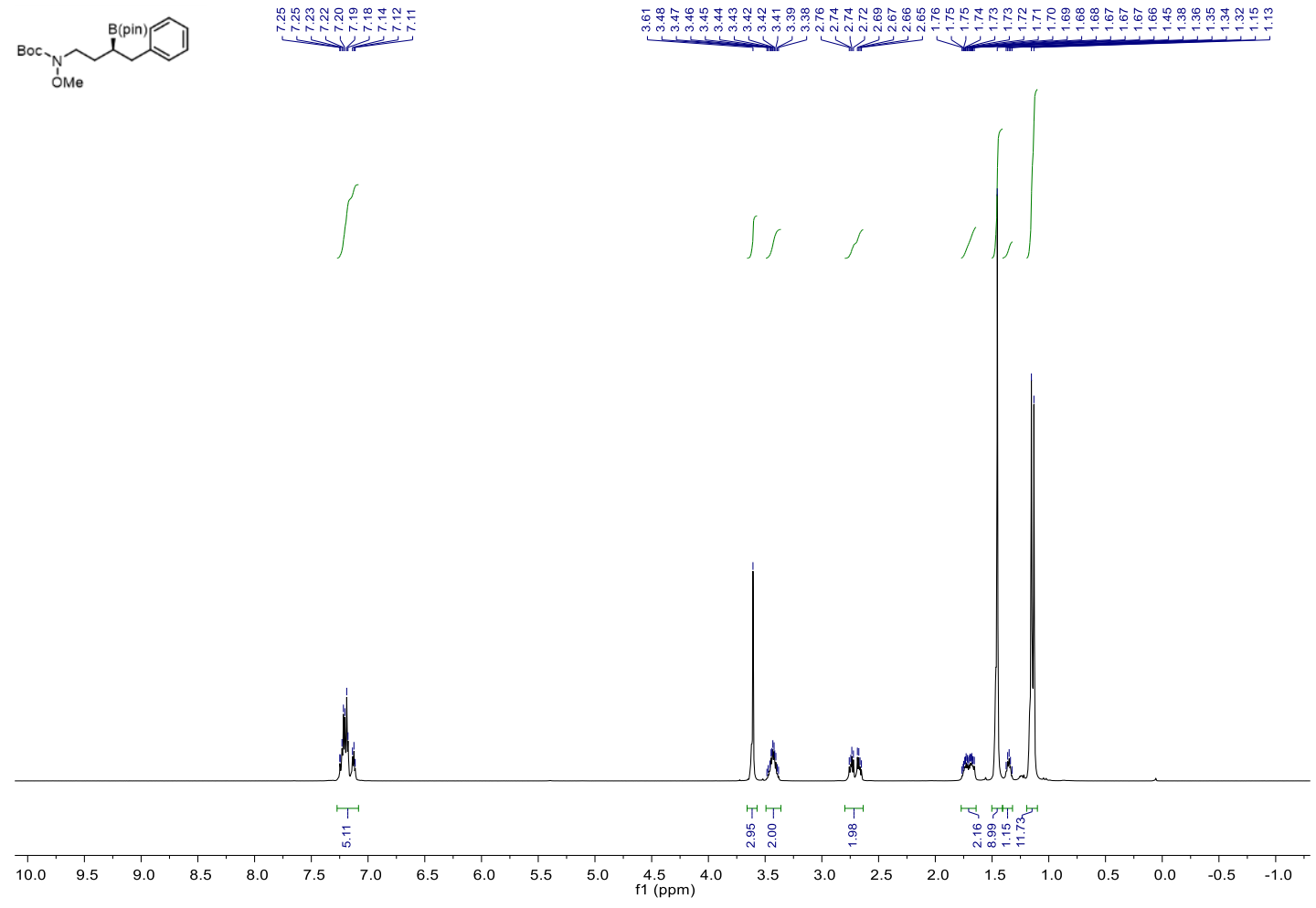

${ }^{13}$ C NMR (151 MHz, $\left.\mathrm{CDCl}_{3}\right)$ (23)
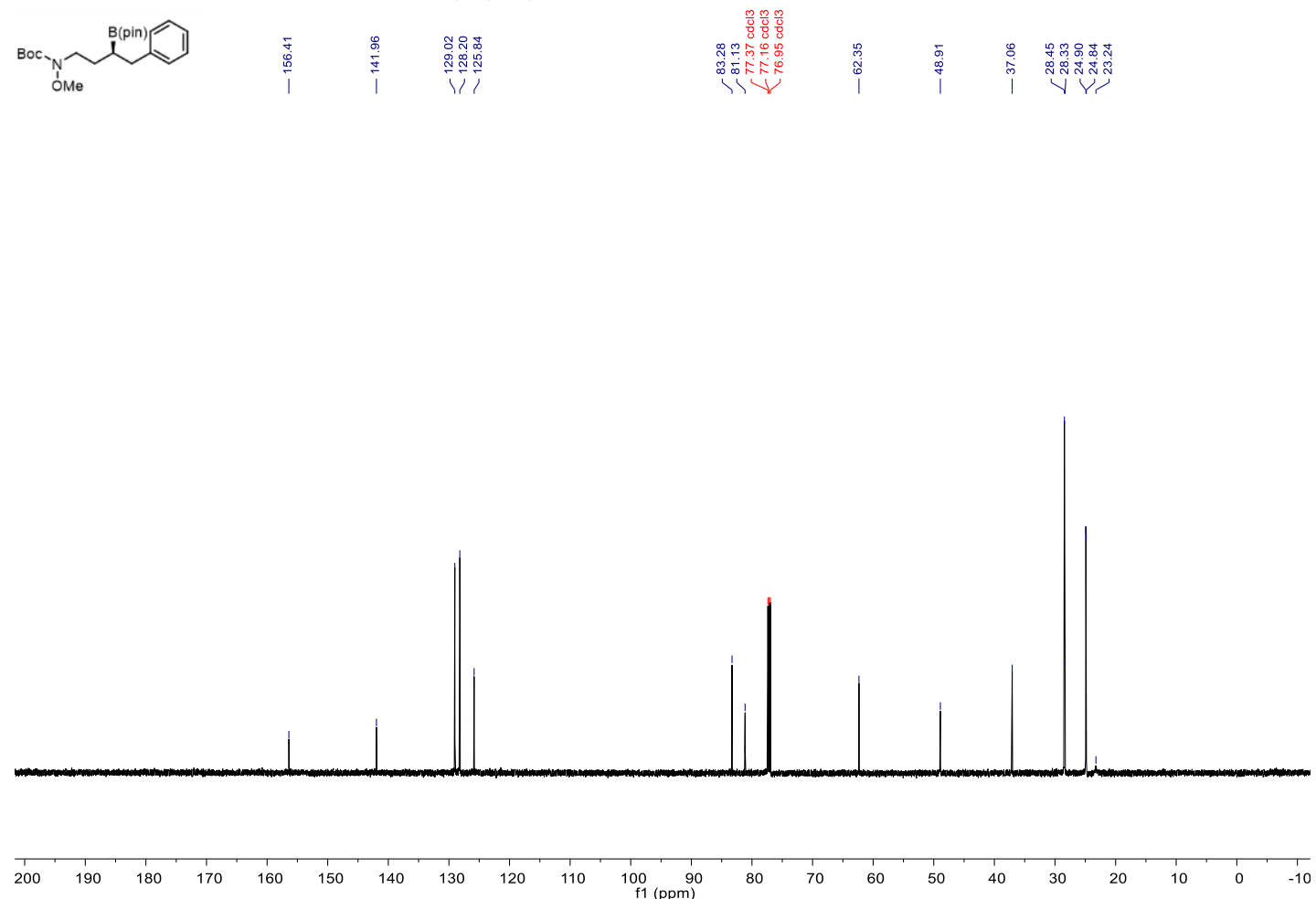
Xu, Zhang, Ingoglia, Allais, Dechert-Schmitt, Singer and Morken Supporting Information

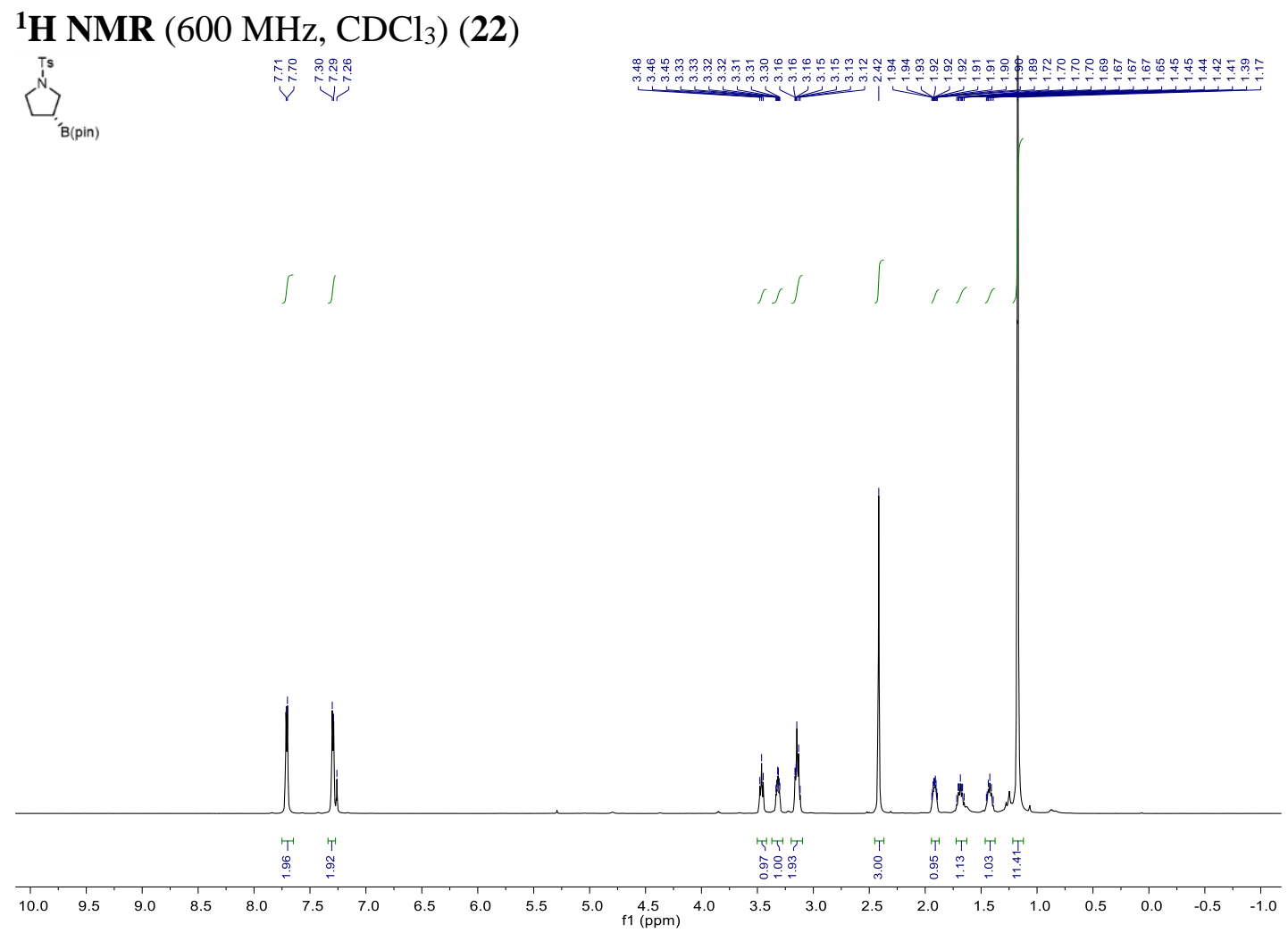

${ }^{13}$ C NMR (151 MHz, $\mathrm{CDCl}_{3}$ ) (22)
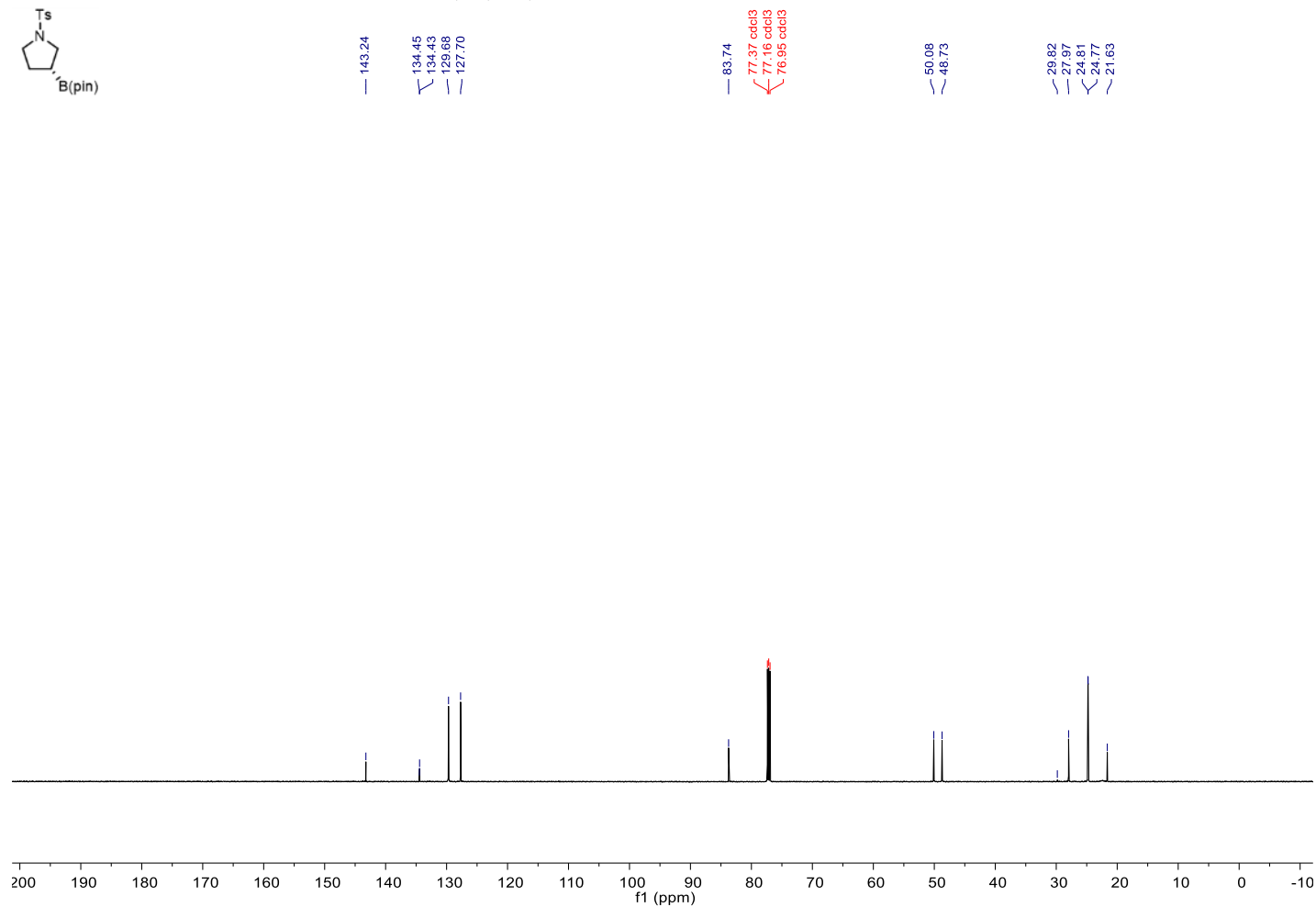
Xu, Zhang, Ingoglia, Allais, Dechert-Schmitt, Singer and Morken Supporting Information

${ }^{\mathbf{1}} \mathbf{H} \mathbf{N M R}\left(600 \mathrm{MHz}, \mathrm{CDCl}_{3}\right)(\mathbf{2 7})$

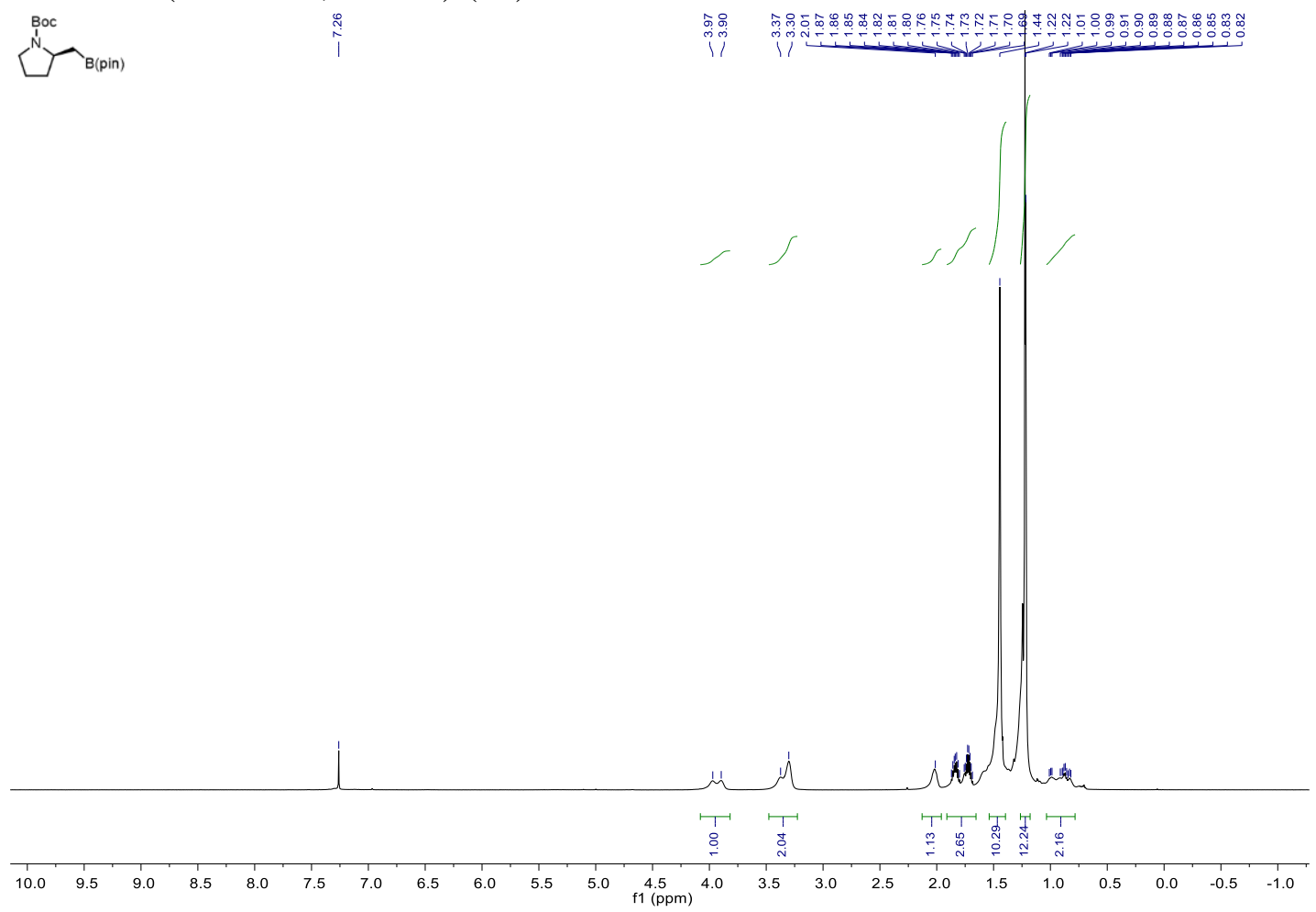

${ }^{13} \mathrm{C}$ NMR (151 MHz, $\left.\mathrm{CDCl}_{3}\right)$ (27)

$\bigsqcup^{\text {Boc }} \Upsilon_{\text {B(pin) }}$ 


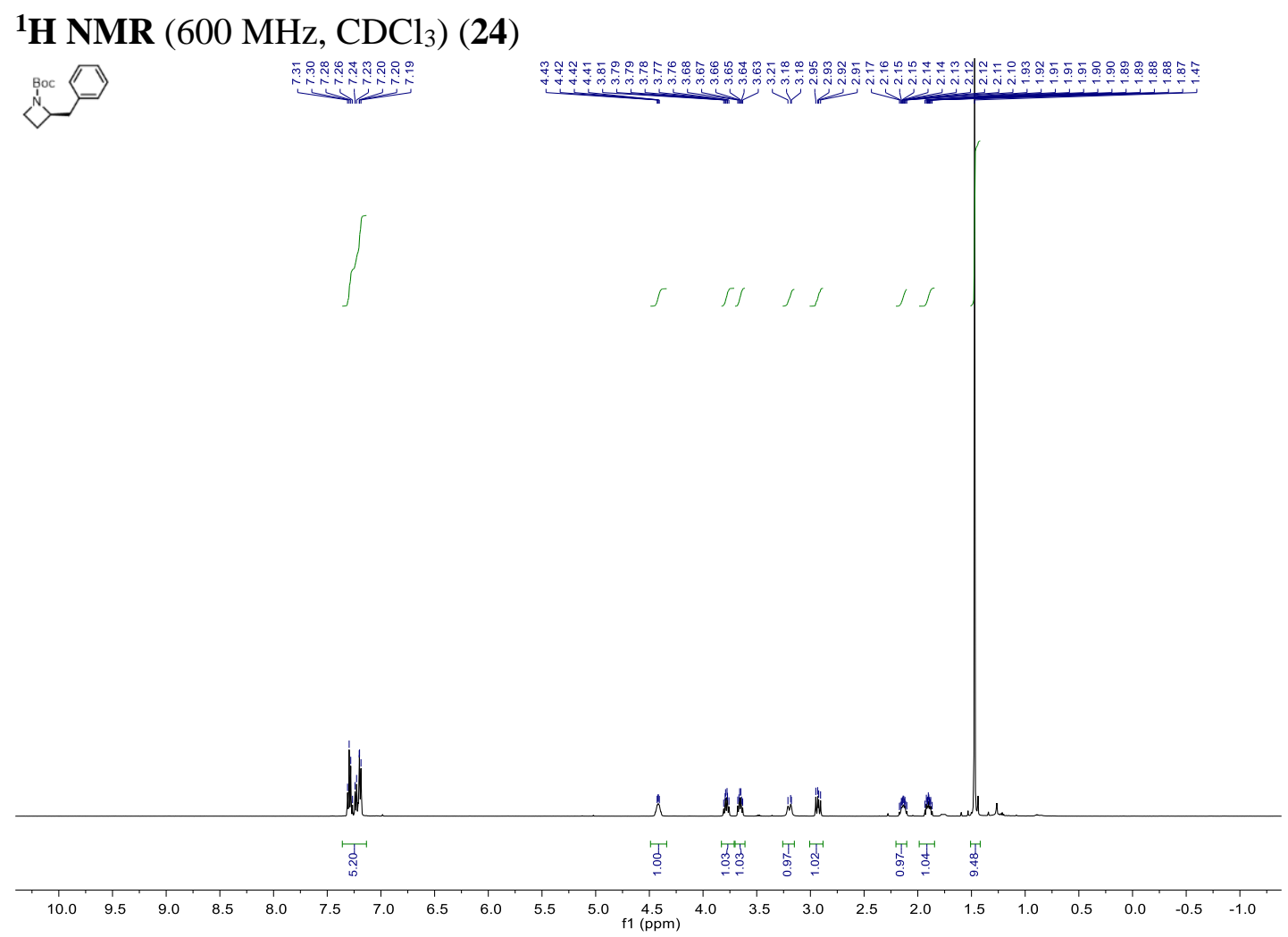

${ }^{13} \mathrm{C}$ NMR (151 MHz, $\left.\mathrm{CDCl}_{3}\right)$ (24)
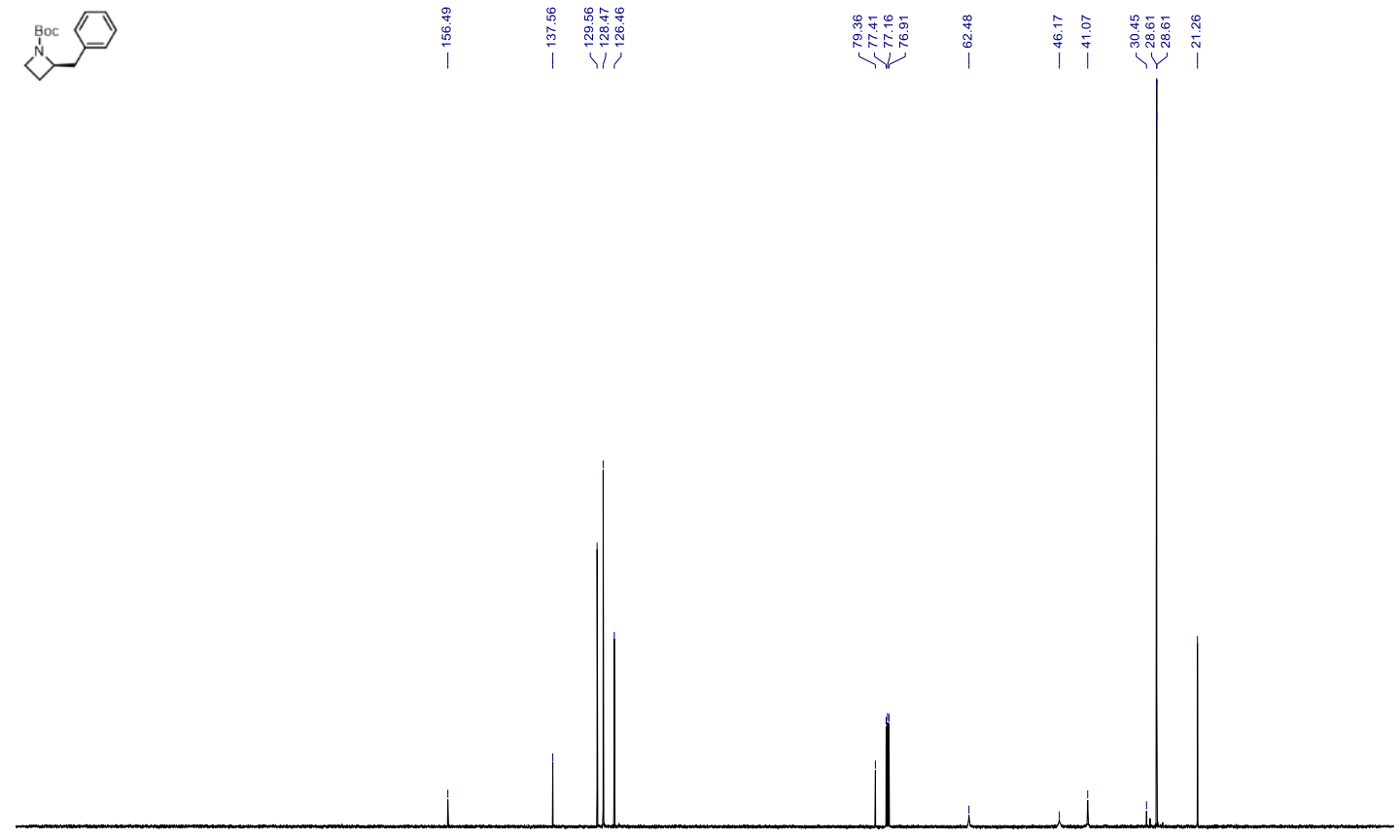

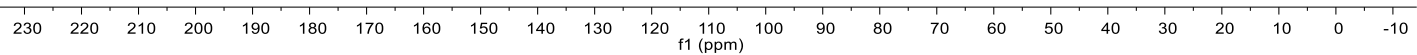

